\title{
Searching for trans ethyl methyl ether in Orion $\mathrm{KL}^{\star, \star \star}$
}

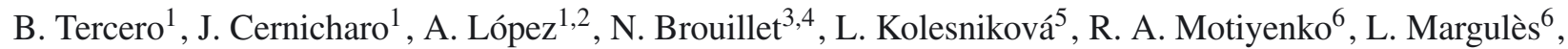 \\ J. L. Alonso ${ }^{5}$, and J.-C. Guillemin ${ }^{7}$ \\ ${ }^{1}$ Grupo de Astrofísica Molecular. Instituto de CC. de Materiales de Madrid (ICMM-CSIC). Sor Juana Inés de la Cruz, 3, Cantoblanco, \\ 28049 Madrid, Spain \\ e-mail: b.tercero@icmm.csic.es; jose.cernicharo@csic.es \\ 2 Dpto. de Astrofísica, CAB, INTA-CSIC, Crta Torrejón-Ajalvir, km 4, 28850 Torrejón de Ardoz, Madrid, Spain \\ 3 Université Bordeaux, LAB, UMR 5804, 33270 Floirac, France \\ ${ }^{4}$ CNRS, LAB, UMR 5804, 33270 Floirac, France \\ ${ }^{5}$ Grupo de Espectroscopía Molecular (GEM), Edificio Quifima, Área de Química-Física, Laboratorios de Espectroscopía y \\ Bioespectroscopía, Parque Científico UVa, Unidad Asociada CSIC, Universidad de Valladolid, 47011 Valladolid, Spain \\ ${ }^{6}$ Laboratoire de Physique des Lasers, Atomes, et Molécules, UMR CNRS 8523, Université de Lille I, \\ 59655 Villeneuve d'Ascq Cédex, France \\ 7 Institut des Sciences Chimiques de Rennes, École Nationale Supérieure de Chimie de Rennes, CNRS, UMR 6226, \\ 11 Allée de Beaulieu, CS 50837, 35708 Rennes Cedex 7, France
}

Received 3 April 2015 / Accepted 16 August 2015

\section{ABSTRACT}

\begin{abstract}
We report on the tentative detection of trans ethyl methyl ether (tEME), $\mathrm{t}-\mathrm{CH}_{3} \mathrm{CH}_{2} \mathrm{OCH}_{3}$, through the identification of a large number of rotational lines from each one of the spin states of the molecule towards Orion KL. We also search for gauche-trans-n-propanol, Gt-n- $\mathrm{CH}_{3} \mathrm{CH}_{2} \mathrm{CH}_{2} \mathrm{OH}$, an isomer of tEME in the same source. We have identified lines of both species in the IRAM $30 \mathrm{~m}$ line survey and in the ALMA Science Verification data. We have obtained ALMA maps to establish the spatial distribution of these species. Whereas tEME mainly arises from the compact ridge component of Orion, Gt-n-propanol appears at the emission peak of ethanol (south hot core). The derived column densities of these species at the location of their emission peaks are $\leq(4.0 \pm 0.8) \times 10^{15} \mathrm{~cm}^{-2}$ and $\leq(1.0 \pm 0.2) \times 10^{15} \mathrm{~cm}^{-2}$ for tEME and Gt-n-propanol, respectively. The rotational temperature is $\sim 100 \mathrm{~K}$ for both molecules. We also provide maps of $\mathrm{CH}_{3} \mathrm{OCOH}, \mathrm{CH}_{3} \mathrm{CH}_{2} \mathrm{OCOH}, \mathrm{CH}_{3} \mathrm{OCH}_{3}, \mathrm{CH}_{3} \mathrm{OH}$, and $\mathrm{CH}_{3} \mathrm{CH}_{2} \mathrm{OH}$ to compare the distribution of these organic saturated O-bearing species containing methyl and ethyl groups in this region. Abundance ratios of related species and upper limits to the abundances of non-detected ethers are provided. We derive an abundance ratio $N\left(\mathrm{CH}_{3} \mathrm{OCH}_{3}\right) / N(\mathrm{tEME}) \geq 150$ in the compact ridge of Orion.
\end{abstract}

Key words. ISM: abundances - ISM: clouds - ISM: individual objects: Orion KL - ISM: molecules - radio lines: ISM - surveys

\section{Introduction}

The spectral millimeter-wave survey of Orion KL carried out with the IRAM $30 \mathrm{~m}$ radio telescope (Tercero et al. 2010; Tercero 2012) shows more than 15400 spectral features of which about 11000 have been identified and attributed to 50 molecules (199 different isotopologues and vibrational modes). To date, there have been several works based on these data. As the result of a fruitful collaboration with spectroscopy laboratories, 3000 previously unidentified lines have been assigned to new species in the interstellar medium (ISM). We have detected in space 16 new isotopologues and vibrationally excited states of

* This paper makes use of the following ALMA data: ADS/JAO.ALMA\#2011.0.00009.SV. ALMA is a partnership of ESO (representing its member states), NSF (USA), and NINS (Japan) with NRC (Canada), NSC, and ASIAA (Taiwan), and KASI (Republic of Korea), in cooperation with the Republic of Chile. The Joint ALMA Observatory is operated by ESO, AUI/NRAO, and NAOJ. This work was also based on observations carried out with the IRAM 30-m telescope. IRAM is supported by INSU/CNRS (France), MPG (Germany), and IGN (Spain).

$\star \star$ Appendix A is available in electronic form at

http://www. aanda.org abundant molecules in Orion for the first time (Demyk et al. 2007; Margulès et al. 2009, 2010; Carvajal et al. 2009; Tercero et al. 2012; Motiyenko et al. 2012; Daly et al. 2013; Coudert et al. 2013; Haykal et al. 2014; López et al. 2014) as well as four new molecules (Tercero et al. 2013; Cernicharo et al. 2013; Kolesniková et al. 2014). These identifications reduce the number of unidentified lines and mitigate line confusion in the spectra. Nevertheless, many features still remain unidentified and correspond to new species that we have to search and identify. Formates, ethers, acetates, alcohols, and cyanides are the best candidates for this purpose in Orion.

The recent search for trans ethyl methyl ether (tEME) in selected hot cores (Sgr B2(N-LMH) and W51 e1/e2) by Carroll et al. (2015) only provides upper limits to tEME. Hence, the results from that work do not confirm the previous tentative identification of this species by Fuchs et al. (2005) towards W51 e1/e2.

A systematic line survey with most weeds removed permits us to address the problem of the abundances of isomers and derivatives of key species, such as methyl formate (A. López et al., in prep.), through combined IRAM and ALMA studies.

In this Letter, we report on the tentative detection of tEME towards the compact ridge (CR) of Orion KL. We have detected emission of features arising from the five spin states at 3,2, and 
$1 \mathrm{~mm}$ with the IRAM $30 \mathrm{~m}$ telescope and the ALMA interferometer. In addition, several unidentified lines of these data have been identified as belonging to the gauche-trans conformer of n-propanol (an isomer of tEME). ALMA maps of organic saturated O-bearing species containing methyl, ethyl, and propyl groups, abundance ratios of related species, and upper limits to the column densities of non-detected ethers are presented and discussed in Sect. 4.

\section{Observations}

IRAM $30 \mathrm{~m}$ : new data of the IRAM $30 \mathrm{~m}$ telescope, which complement and improve those of Tercero et al. (2010), were collected in August 2013 and March 2014 towards Orion KL (see Tercero et al. 2010 and López et al. 2014, for information about the previous data set). Frequencies in the ranges 80.7-116, 122.7-161.2, 199.7-291.0, 291.4-306.7 GHz, were observed with the EMIR receivers connected to the FFTS $(200 \mathrm{kHz}$ of spectral resolution) spectrometers. We pointed towards IRc2 source at $\alpha_{2000.0}=5^{\mathrm{h}} 35^{\mathrm{m}} 14.5, \delta_{2000.0}=-5^{\circ} 22^{\prime} 30^{\prime} .0$, corresponding to the survey position (see Sect.4). We observed an additional position to target the CR: $\alpha_{2000.0}=5^{\mathrm{h}} 35^{\mathrm{m}} 14^{\mathrm{s}} .3$, $\delta_{2000.0}=-5^{\circ} 22^{\prime} 37^{\prime \prime}$.0 (see Sect. 4 ). The observations were performed using the wobbler switching mode with a beam throw in azimuth of $\pm 120^{\prime \prime}$. The intensity scale was calibrated using the atmospheric transmission model (ATM, Cernicharo 1985; Pardo et al. 2001). Focus and pointing were checked every $1-2 \mathrm{~h}$ on planets or nearby quasars. System temperatures were in the range of 100-800 K from the lowest to highest frequencies. Half power beam width (HPBW) ranged from 31" to $8^{\prime \prime}$ from 80 to $307 \mathrm{GHz}(H P B W[\operatorname{arcsec}]=2460 /$ Freq. $[\mathrm{GHz}])$. The data were reduced using the GILDAS package ${ }^{1}$.

ALMA SV: the ALMA Science Verification (SV) data ${ }^{2}$ were taken in January 2012 towards the IRc2 region in Orion. The observations were carried out with 16 antennas of $12 \mathrm{~m}$ in Band 6 (213.715-246.627 GHz). The primary beam was $\simeq 27^{\prime \prime}$. Spectral resolution was $0.488 \mathrm{MHz}$ corresponding to a velocity resolution of $0.64 \mathrm{~km} \mathrm{~s}^{-1}$. The observations were centred on coordinates: $\alpha_{\mathrm{J} 2000}=05^{\mathrm{h}} 35^{\mathrm{m}} 14.35, \delta_{\mathrm{J} 2000}=-05^{\circ} 22^{\prime} 35^{\prime \prime} \cdot 00$. The CASA software $^{3}$ was used for initial processing and then the visibilities were exported to the GILDAS package. The line maps were cleaned using the HOGBOM algorithm (Högbom 1974). The synthesized beam ranged from $2^{\prime \prime}$. $00 \times 1^{\prime \prime}$. 48 with a PA of $176^{\circ}$ at $214.0 \mathrm{GHz}$ to $1^{\prime \prime} .75 \times 11^{\prime \prime} .29$ with a PA of $164^{\circ}$ at $246.4 \mathrm{GHz}$. The brightness temperature to flux density conversion factor is $9 \mathrm{~K}$ for $1 \mathrm{Jy}$ per beam.

\section{Results}

\subsection{Search for trans ethyl methyl ether}

ALMA SV data: frequency predictions from Fuchs et al. (2003) and dipole moments measured by Hayashi \& Kuwada (1975) of tEME were implemented in MADEX (Cernicharo 2012) to model the emission of this species and search for it towards Orion KL. Using the ALMA SV data, we extracted the averaged spectrum over $5 \times 5$ pixels $\left(1^{\prime \prime} \times 1^{\prime \prime}\right)$ around the $\mathrm{CH}_{3} \mathrm{OCH}_{3}$ emission peak of the CR component (Position A; see Sect.4). The advantage of ALMA with respect to single dish telescope data (see below) is the drastic reduction of the confusion limit.

\footnotetext{
1 http://www.iram.fr/IRAMFR/GILDAS

2 http://almascience.eso.org/almadata/sciver/ OrionKLBand6/

3 http://casa.nrao.edu
}
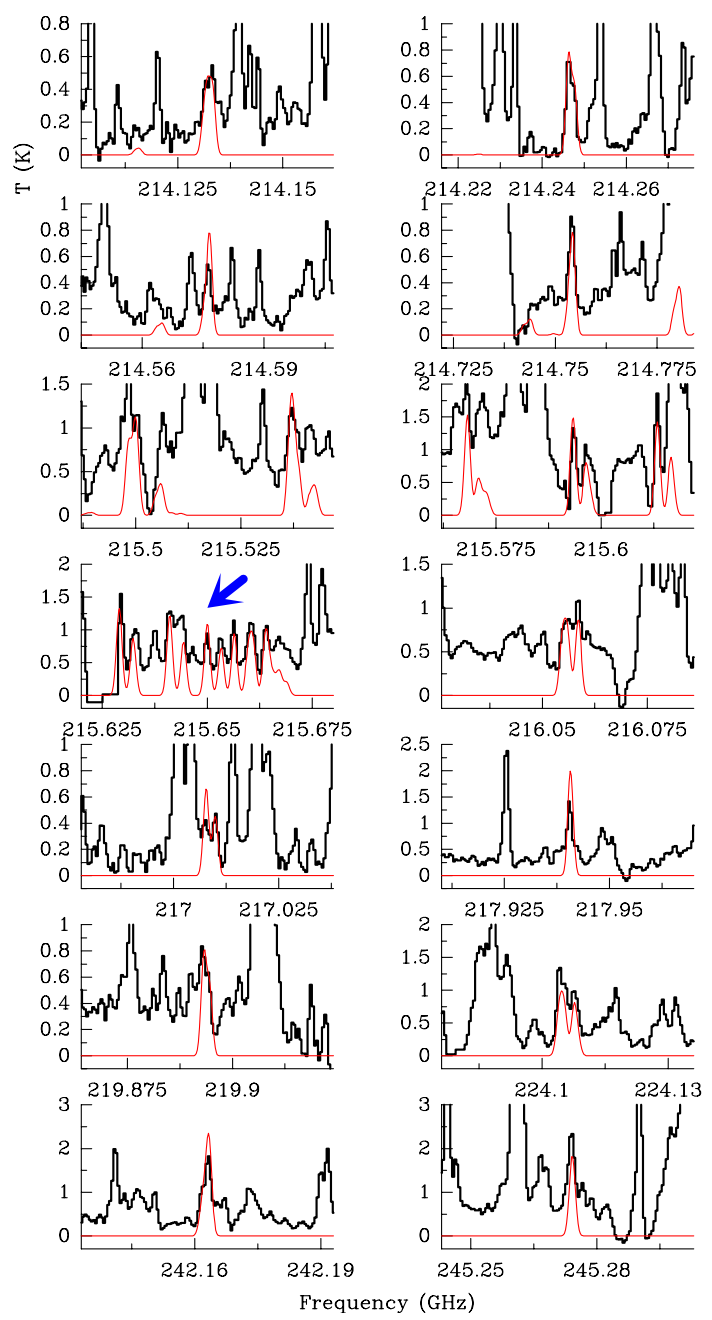

Fig. 1. Selected lines of trans ethyl methyl ether, $t-\mathrm{CH}_{3} \mathrm{CH}_{2} \mathrm{OCH}_{3}$, towards Orion KL detected with the ALMA interferometer in Position A (see text). A $v_{\text {LSR }}$ of $+7.5 \mathrm{~km} \mathrm{~s}^{-1}$ is assumed.

The ALMA SV data show the presence of tEME as shown in Fig. 1 (selected lines) and Fig. A.1 (all lines favourable for detection (corresponding to $b$-type transitions with upper level energies up to $300 \mathrm{~K}$ and large line strenghts, $S_{i j} \geq 1$ ) present in the ALMA SV frequency range). The model that best fits the data is shown with the red line. The assumed parameters are a source size of $3^{\prime \prime}, v_{\mathrm{LSR}}=+7.5 \mathrm{~km} \mathrm{~s}^{-1}, \Delta v=2.0 \mathrm{~km} \mathrm{~s}^{-1}$, and $T_{K}=100 \pm 20 \mathrm{~K}$. Using MADEX and assuming local thermodynamic equilibrium (LTE), we obtain $N_{\text {g.s. }}$ (tEME) $\leq$ $(4.0 \pm 0.8) \times 10^{15} \mathrm{~cm}^{-2}$. In our models, rotation temperature and column density values are given with their corresponding uncertainty and we obtained them by fitting all available lines by eye. We adopted the source size in agreement with the emission of the maps (see below). In addition, a considerable number of unblended features allows us to fix the radial velocities and line widths. According to our model, in the ALMA frequency range only $33 \%$ of the detectable lines of tEME (102 lines) are totally hidden by the emission of stronger lines of other species. At least 46 lines (45\% of the detectable lines) shown in Fig. A.1 are free of blending, i.e. these lines are present at the expected radial velocity and there are no other species with significant intensity at the same observed frequency $( \pm 3 \mathrm{MHz})$. Another point to ensure this tentative detection is that the forest of lines emitted by tEME between 215.5 and $215.7 \mathrm{GHz}$ is not covered by lines of abundant molecules in the source allowing the detection 
of several lines that follow a straightened pattern (see Fig. 1). Hence, there are several clues that could reveal the presence of this species in the CR of Orion KL, but further analysis exploring new available ALMA data and modelling all the molecular content of the CR is needed to give the definitive detection in space of tEME. Table A.1 gives line parameters and blends of all lines of favourable transitions in the ALMA SV data. The spatial distribution of tEME is shown in Fig. 2. Lines that we found to be unblended at the Position A appear blended with emission from other components in the averaged spectrum (see the case of the $30 \mathrm{~m}$ data). We selected a line at $245.274 \mathrm{GHz}$, which is mixed with some emission from extreme velocities of ${ }^{34} \mathrm{SO}_{2}$ and $\mathrm{SO}_{2}$. Nevertheless, the emission of tEME at Position A in Fig. 2 is not blended (see Sect. 4).

IRAM $30 \mathrm{~m}$ data: to search for tEME in the IRAM data, a synthetic spectra of tEME (red curve in Fig. A.2) was obtained with MADEX assuming LTE and adopting the following physical parameters: source diameter $3^{\prime \prime}, T_{K}=100 \pm 30 \mathrm{~K}$, $v_{\mathrm{LSR}}=+7.5 \mathrm{~km} \mathrm{~s}^{-1}, \Delta v=1.5 \mathrm{~km} \mathrm{~s}^{-1}$; and a column density of $(9 \pm 3) \times 10^{15} \mathrm{~cm}^{-2}$ for the ground state (g.s.) of tEME. According to our model, all favourable lines for detection in the $30 \mathrm{~m}$ data were detected or were blended with features from more abundant species. Nevertheless, owing to the weakness of the features $\left(T_{\mathrm{MB}}<0.1 \mathrm{~K}\right.$ at $3 \mathrm{~mm}, T_{\mathrm{MB}}<0.2 \mathrm{~K}$ at $2 \mathrm{~mm}$, and $T_{\mathrm{MB}}<1 \mathrm{~K}$ at $1.3-0.9 \mathrm{~mm}$ ) and the high level of line confusion at $\sim 1 \mathrm{~mm}$, only a few lines were mostly free of blending with other species in this domain. Whereas the synthetic beam of the ALMA SV is $1^{\prime \prime} .90 \times 11^{\prime \prime} 40$, in the $30 \mathrm{~m}$ the beam diameter ranging from $30^{\prime \prime}$ to $8^{\prime \prime}$. Therefore, in the $30 \mathrm{~m}$ data, the spectrum is a mix of all molecules from all source components (average spectrum over the beam) given rise to a high level of line blending and line confusion. Table A.2 shows line parameters, intensity provided by the model, and blends of all lines of favourable transitions in the $30 \mathrm{~m}$ data.

\subsection{Search for gauche-trans-n-propanol}

All lines of $\mathrm{Gt}-\mathrm{CH}_{3} \mathrm{CH}_{2} \mathrm{CH}_{2} \mathrm{OH}$, an isomer of $\mathrm{C}_{3} \mathrm{H}_{8} \mathrm{O}$ (as well as tEME), reported by Maeda et al. (2006) and the dipole moments from Abdurakhmanov et al. (1969) were used to derive its rotational constants and to implement this species in MADEX. We conducted the search for Gt-n-propanol in the ALMA SV data at two different positions: Position A and the position where the emission peak of ethanol is located (Position B; see Sect. 4). We assign several unidentified lines in the source at Position B to this species. According to our model $\left(d_{\text {sou }}=3^{\prime \prime}\right.$, $v_{\mathrm{LSR}}=+8.0 \mathrm{~km} \mathrm{~s}^{-1}, \Delta v=3.0 \mathrm{~km} \mathrm{~s}^{-1}, T_{K}=100 \pm 20 \mathrm{~K}$, and $\left.N_{\text {g.s }} \leq(1.0 \pm 0.2) \times 10^{15} \mathrm{~cm}^{-2}\right)$, many of the lines are below the detection limit although the strongest features are detected. Unfortunately, several lines remain blended (see Fig. A.3). A few lines of this species are also detected in the IRAM $30 \mathrm{~m}$ data at the survey position (Fig. A.2 bottom panel; model parameters: $d_{\text {sou }}=3^{\prime \prime}, v_{\mathrm{LSR}}=+8.0 \mathrm{~km} \mathrm{~s}^{-1}, \Delta v=1.5 \mathrm{~km} \mathrm{~s}^{-1}$, $T_{K}=100 \pm 20 \mathrm{~K}$, and $\left.N_{\text {g.s }} \leq(2.0 \pm 0.4) \times 10^{15} \mathrm{~cm}^{-2}\right)$. Table A.3 shows line parameters for the detected lines. The derived upper limit to its column density (assuming the same physical parameters than those of the tEME ALMA model) at Position $\mathrm{A}$ is $\leq(3.0 \pm 0.6) \times 10^{14} \mathrm{~cm}^{-2}$. The spatial distribution of this species around Position B is shown in Fig. 2. To perform the ALMA map, we averaged the emission between $v_{\mathrm{LSR}} 6$ and $9 \mathrm{~km} \mathrm{~s}^{-1}$ of two lines (lines at 236.138 and $244.765 \mathrm{GHz}$ ). Emission around source $I$ should be due to other less abundant species in Orion (we did not find Gt-n-propanol at these positions).

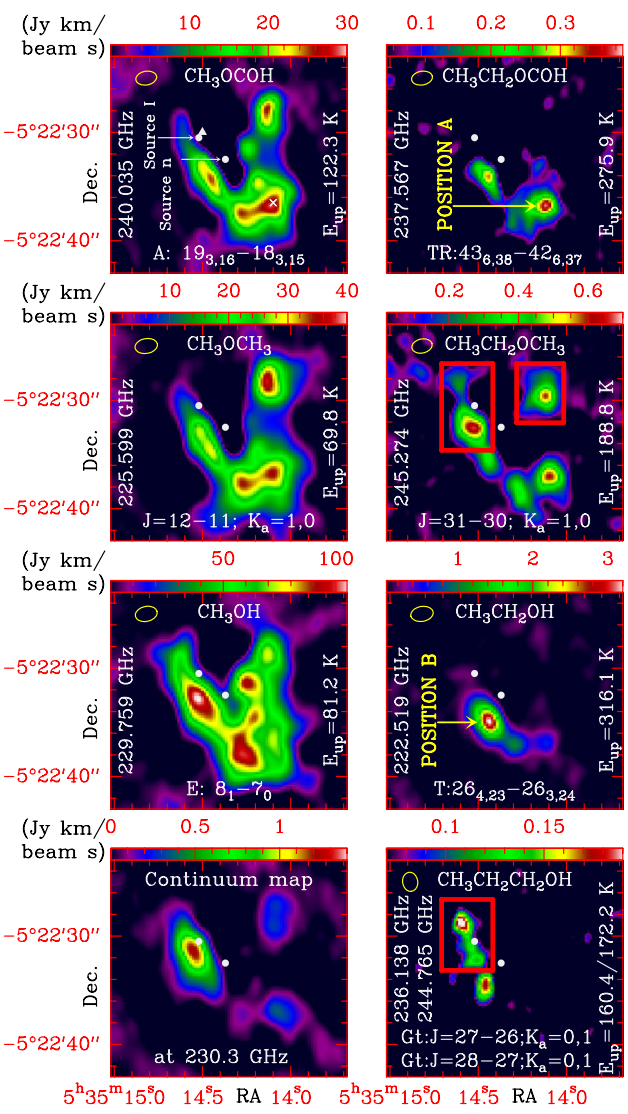

Fig. 2. ALMA maps of organic saturated O-bearing molecules in Orion KL which have been detected containing both the methyl and the ethyl group, as well as a map of Gt-n-propanol and a continuum map at the central frequencies of the ALMA $S V$ band $(\sim 230 \mathrm{GHz})$. Emission that probably arises from blended species in these maps is confined inside red rectangles. The yellow ellipse at the top left corner of the maps represents the ALMA synthetic beam. Triangle symbol: IRAM 30 m "survey position" (see Sect. 2). Cross symbol: IRAM 30 m compact ridge position (see Sect. 2). Position A: compact ridge (coordinates $\left.\alpha_{2000.0}=5^{\mathrm{h}} 35^{\mathrm{m}} 14 \mathrm{~s} .1, \delta_{2000.0}=-5^{\circ} 22^{\prime} 37^{\prime \prime} .9\right)$. Position B: south hot core (coordinates $\alpha_{2000.0}=5^{\mathrm{h}} 35^{\mathrm{m}} 14.4, \delta_{2000.0}=-5^{\circ} 22^{\prime} 34^{\prime \prime} .9$ ).

\section{Discussion}

Species containing the functional groups formate, alcohol, and ether have been detected in Orion with both the methyl and ethyl groups (methyl formate (MF), ethyl formate (EF), methanol, ethanol, dimethyl ether (DME), and tEME). ALMA maps for the spatial distribution of these species as well as Gt-n-propanol are shown in Fig. 2. To address the flux filtered out by ALMA and the accuracy of the maps in a larger energy range, the following discussion is also based on the maps shown in Fig. 5 of Feng et al. (2015; maps performed mixing SMA and IRAM $30 \mathrm{~m}$ data) with MF, DME, methanol, and ethanol. For MF, DME, and methanol the spatial distribution and the position of the emission peaks are in agreement with those of the maps presented in this work (note, however, that the ALMA maps provide a more detailed structure at small scales, i.e. $\leq 5^{\prime \prime}$ ). For ethanol, we note a more extended spatial distribution in the map of Feng et al. (2015) mostly due to the lower energy of the transition involved. Nevertheless, the emission peak of ethanol is located at the same position.

For the methyl species, we note: i) a rather similar spatial structure: the three species present the V shape distribution of several clumps (at least six) studied by Favre et al. (2011) for the distribution of MF, which was mapped using data from the 
Table 1. Column densities and ratios.

\begin{tabular}{|c|c|c|}
\hline Species & $N_{\text {g.s. }}\left(\times 10^{15}\right)\left[\mathrm{cm}^{-2}\right]$ & $N$ Ratio \\
\hline $\mathrm{CH}_{3} \mathrm{OCH}_{3}(\mathrm{DME})$ & $600 \pm 120^{a, b}$ & \\
\hline $\mathrm{CH}_{3} \mathrm{CH}_{2} \mathrm{OCH}_{3}$ (tEME) & $\leq 4.0 \pm 0.8^{a}$ & $\mathrm{DME} / \mathrm{tEME} \geq 150$ \\
\hline $\begin{array}{l}\mathrm{CH}_{3} \mathrm{CH}_{2} \mathrm{OCH}_{2} \mathrm{CH}_{3} \\
(\mathrm{Tt}-\mathrm{DEE})^{\dagger}\end{array}$ & $\leq 1.0 \pm 0.2^{a}$ & $\begin{array}{l}\mathrm{DME} / \mathrm{Tt}-\mathrm{DEE} \geq 600 \\
\text { tEME/Tt-DEE } \geq 4\end{array}$ \\
\hline $\begin{array}{l}\mathrm{CH}_{3} \mathrm{OCHCH}_{2} \\
\left(\text { cis-MVE) }{ }^{\dagger \dagger}\right.\end{array}$ & $\leq 0.5 \pm 0.1^{a}$ & $\begin{array}{l}\mathrm{DME} / \text { cis-MVE } \geq 1200 \\
\text { tEME/cis-MVE } \geq 9\end{array}$ \\
\hline $\mathrm{CH}_{3} \mathrm{OCOH}(\mathrm{MF})$ & $240 \pm 50^{a, b, c}$ & \\
\hline $\begin{array}{l}\mathrm{CH}_{3} \mathrm{CH}_{2} \mathrm{OCOH}(\mathrm{EF}) \\
\mathrm{CH}_{3} \mathrm{OH}(\mathrm{MetOH})\end{array}$ & $\begin{array}{l}2.0 \pm 0.4^{a, d} \\
2700 \pm 500^{b, e, f}\end{array}$ & $\mathrm{MF} / \mathrm{EF} \simeq 120$ \\
\hline $\mathrm{CH}_{3} \mathrm{CH}_{2} \mathrm{OH}(\mathrm{EtOH})$ & $60 \pm 10^{b, d, e}$ & $\mathrm{MetOH} / \mathrm{EtOH} \simeq 45$ \\
\hline $\begin{array}{l}\mathrm{Gt}_{-} \mathrm{CH}_{3} \mathrm{CH}_{2} \mathrm{CH}_{2} \mathrm{OH} \\
\text { (PropOH) }\end{array}$ & $1.0 \pm 0.2^{e}$ & $\begin{array}{l}\text { MetOH/PropOH } \simeq 2700 \\
\text { EtOH/PropOH } \simeq 60\end{array}$ \\
\hline
\end{tabular}

Notes. ${ }^{(\dagger)}$ trans-trans diethyl ether. ${ }^{(\dagger \dagger)}$ cis methyl vinyl ether. (a) Position A; same physical parameters of the ALMA tEME model (see Sect. 3.1). ${ }^{(b)}$ Three kinetic temperatures: $50 \pm 10,150 \pm 30$, and $250 \pm 75 \mathrm{~K} .{ }^{(c)}$ b type lines fitted (a type lines are optically thick); another component has been included to properly fit the observed line profiles $\left(v_{\mathrm{LSR}}=+9 \mathrm{~km} \mathrm{~s}^{-1}, \Delta v=4 \mathrm{~km} \mathrm{~s}^{-1}, T_{K}=150 \pm 30 \mathrm{~K}, N_{\mathrm{g} . \mathrm{s}}=\right.$ $\left.(1.0 \pm 0.2) \times 10^{17} \mathrm{~cm}^{-2}\right)$. ${ }^{(d)}$ trans + gauche. ${ }^{(e)}$ Position $\mathrm{B}$; assuming the same physical parameters of the ALMA Gt-n-propanol model (see Sect. 3.2). ${ }^{(f)}{ }^{12} \mathrm{C} /{ }^{13} \mathrm{C}=45$ (Tercero et al. 2010).

Plateau de Bure Interferometer (PdBI); ii) that although Brouillet et al. (2013) probed a striking similarity between the spatial distributions of $\mathrm{CH}_{3} \mathrm{OCH}_{3}$ and $\mathrm{CH}_{3} \mathrm{OCOH}$, we found some differences in the relative intensities of both species. These differences could be mostly due to different excitation temperatures of the involved transitions; and iii) although methanol also follows this $\mathrm{V}$ shape structure, a displacement of the intensity peaks is observed with respect to MF. This behaviour suggests methanol as a possible precursor of MF and DME (see also Neill et al. 2011).

Comparing the methyl and ethyl species, we note: i) a reduced spatial distribution of the three ethyl species with respect to their methyl counterpart; ii) the two emission peaks of EF are correlated with those found in MF; iii) the emission peak of tEME is at the same position as the DME peak at the CR (Position A); and iv) the emission peak of ethanol (Position B) is displaced $2^{\prime \prime}$ south-west from the methanol peak.

Concerning the ethyl and propyl species, we note: i) a close correlation between EF and tEME; and ii) ethanol also presents a "V" shape structure (see Fig. 5 of Feng et al. 2015) with the bulk of the emission located away from the CR and coinciding with that of Gt-n-propanol. The ethanol/propanol peak is displaced 1".5 south from the ethylene glycol $\left(\mathrm{CH}_{2} \mathrm{OH}\right)_{2}$ peak (Brouillet et al. 2015), which is a double alcohol and we could naively expect to have the same spatial distribution. Whereas the ethylene glycol peak corresponds to the ${ }^{13} \mathrm{CH}_{3} \mathrm{OH}$ peak, the ethanol/propanol peak is the same as that of deuterated methanol $\left(\mathrm{CH}_{2} \mathrm{DOH}\right.$; see Peng et al. 2012).

Table 1 shows derived column densities and ratios for related species. The derived ratios and the spatial distribution of these molecules suggest important gas phase processes after the evaporation of the mantles of dust grains in hot cores. Possible reactions of the methoxy radical $\left(\mathrm{CH}_{3} \mathrm{O}\right)$, detected recently in space (Cernicharo et al. 2012), with other species could lead to the increase of chemical complexity in hot cores and hot corinos (Balucani et al. 2015). The spatial stratification of the different species also suggests the time dependent effects on the chemistry of the gas. The detection of the less stable isomers of some species (Tercero et al. 2013) also points in this direction.

To summarize, a combined IRAM $30 \mathrm{~m}$ and ALMA SV data study allows us to provide a solid starting point to assess the identification of tEME in the ISM. In addition, some unidentified lines in the source have been assigned to another $\mathrm{C}_{3} \mathrm{H}_{8} \mathrm{O}$ isomer, Gt-n-propanol. ALMA maps show different spatial distributions for these species. Whereas tEME seems to mainly arises from the CR component (as well as EF) (Position A), emission from Gt-n-propanol could be located at the south hot core (at the same position as the emission peak of ethanol) (Position B). The CR is no longer the main host of all organic saturated O-bearing species in Orion (see also Peng et al. 2013, for the spatial distribution of acetone and A. López et al., in prep. for the acetic acid emission).

Acknowledgements. We thank Marcelino Agúndez for carefully reading the paper and providing useful comments and suggestions. B.T., J.C., and A.L. thank the Spanish MINECO for funding support under grants CSD2009-00038, AYA2009-07304, and AYA2012-32032 and also the ERC for funding support under grant ERC-2013-Syg-610256-NANOCOSMOS.

\section{References}

Abdurakhmanov, A. A., Ragimova, R. A., \& Imanov, L. M. 1969, Opt. Spektrosk., 26, 135 (English transl. in 1969, Opt. Spectrosc., 25, 75) Balucani, N., Ceccarelli, C., \& Taquet, V. 2015, MNRAS, 449, L16 Brouillet, N., Despois, D., Baudry, A., et al. 2013, A\&A, 550, A46 Brouillet, N., Despois, D., Lu, X.-H., et al. 2015, A\&A, accepted Carroll, P. B., McGuire, B. A., Blake, G. A., et al. 2015, ApJ, 799, 15 Carvajal, M., Margulès, L., Tercero, B., et al. 2009, A\&A, 500, 1109 Cernicharo, J. 1985, Internal IRAM Report (Granada: IRAM)

Cernicharo, J. 2012, in ECLA-2011: Proc. Europ. Conf. on Laboratory Astrophysics, eds. C. Stehl, C. Joblin, \& L. d'Hendecourt (Cambridge: Cambridge Univ. Press), EAS PS, 58, 251

Cernicharo, J., Marcelino, N., Roueff, E., et al. 2012, ApJ, 759, L43

Cernicharo, J., Tercero, B., Fuente, A., et al. 2013, ApJ, 771, L10

Coudert, L. H., Drouin, B. J., Tercero, B., et al. 2013, ApJ, 779, 119

Daly, A.M., Bermúdez, C., López, A., et al. 2013, ApJ, 768, 81

Demyk, K., Mäder, H., Tercero, B., et al. 2007, A\&A, 466, 255

Favre, C., Despois, D., Brouillet, N., et al. 2011, A\&A, 532, A32

Feng, S. Y., Beuther, H., Henning, T. 2015, A\&A, accepted

Fuchs, U., Winnewisser, G., Groner, P., De Lucia, F. C., \& Herbst, E. 2003, ApJS, 144,277

Fuchs, G. W., Fuchs, U., Giesen, T. F., \& Wyrowski, F. 2005, A\&A, 444, 521

Hayashi, M., \& Kuwada, K. 1975, J. Mol. Struct., 28, 147

Haykal, I., Carvajal, M., Tercero, B. et al. 2014, A\&A, 568, A58

Högbom, J. A. 1974, A\&AS, 15, 417

Kolesniková, L., Tercero, B., Cernicharo, J., et al. 2014, ApJ, 784, L7

López, A., Tercero, B., Kisiel, Z. et al. 2014, A\&A, 572, A44

Maeda, A., De Lucia, F., C., Herbst, E. et al. 2006, ApJS, 162, 428

Margulès, L., Motiyenko, R. A., Demyk, K., et al. 2009, A\&A, 493, 565

Margulès, L., Huet, T. R., Demaison, J., et al. 2010, ApJ, 714, 1120

Motiyenko, R. A., Tercero B., Cernicharo, J., \& Margulès, L. 2012, A\&A, 548, A71

Neill, J. L., Steber, A. L., Muckle, M. T., et al. 2011, J. Phys. Chem. A, 115, 6472

Pardo, J. R., Cernicharo, \& J., Serabyn, E. 2001, IEEE Trans. Antennas and Propagation, 49, 12

Peng, T.-C., Despois, D., Brouillet, N., Parise, B., \& Baudry, A. 2012, A\&A, 543, A 152

Peng, T.-C., Despois, D., Brouillet, N., et al. 2013, A\&A, 554, A78

Tercero, B. 2012, Ph.D. Thesis, Univ. Complutense de Madrid

Tercero, B., Cernicharo, J., Pardo, J. R., \& Goicoechea, J. R. 2010, A\&A, 517, A96

Tercero, B., Margulès, L., Carvajal, M., et al. 2012, A\&A, 538, A119

Tercero, B., Kleiner, I., Cernicharo, J., et al. 2013, ApJ, 770, L13

Pages 5 to 43 are available in the electronic edition of the journal at http://wWw . aanda. org 
B. Tercero et al.: Trans ethyl methyl ether in space

Appendix A: Online figures and tables

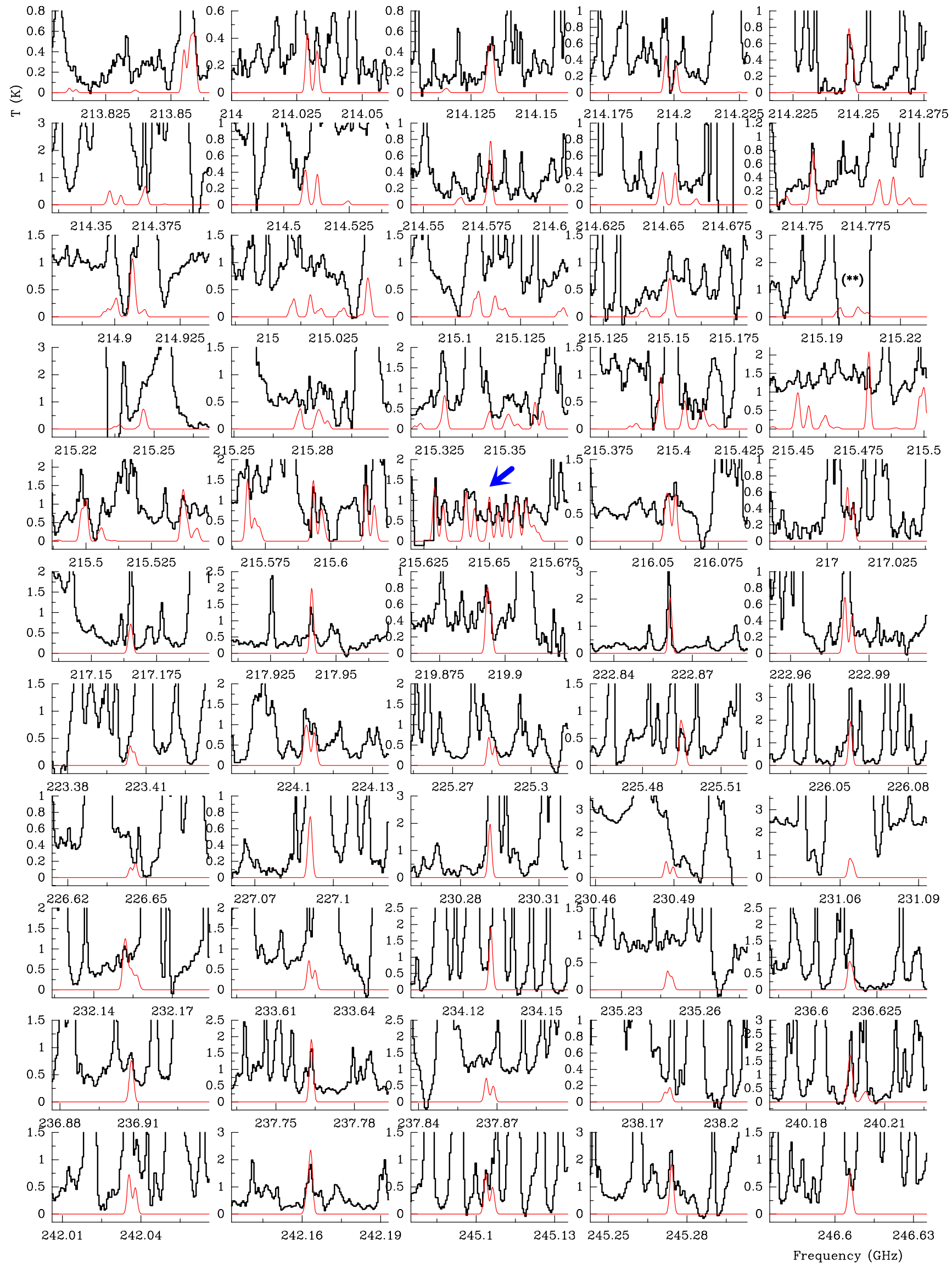

Fig. A.1. Lines of trans ethyl methyl ether, $\mathrm{t}-\mathrm{CH}_{3} \mathrm{CH}_{2} \mathrm{OCH}_{3}$, towards Orion $\mathrm{KL}$ detected with the ALMA interferometer in Position A (see text). (**): Features blended with SO (see Table A.1; artifacts in the spectrum due to the cleaning process). A $v_{\mathrm{LSR}}$ of $+7.5 \mathrm{~km} \mathrm{~s}^{-1}$ is assumed. 

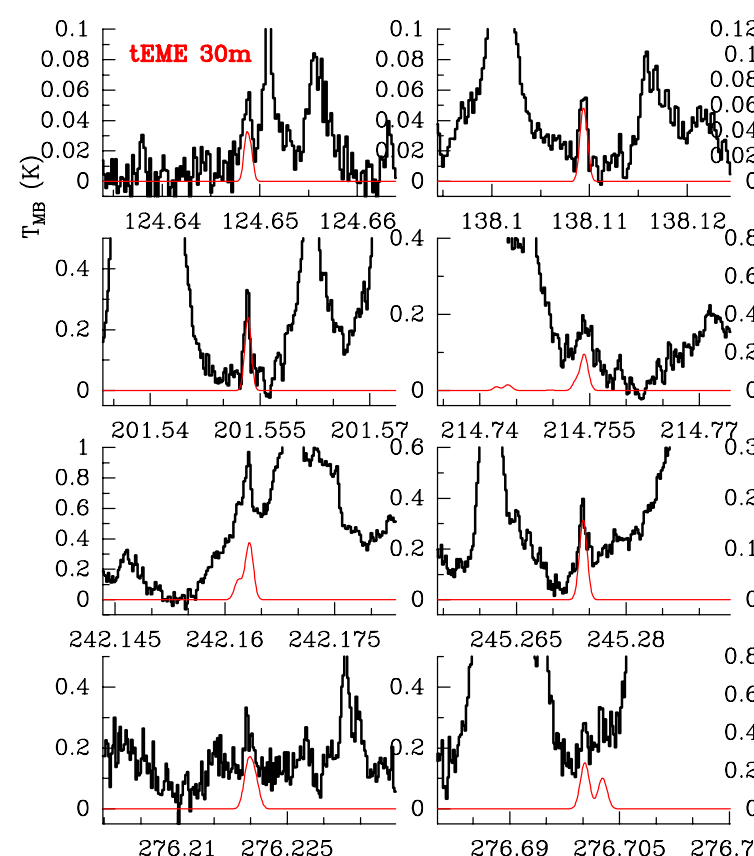

$245.265 \quad 245.28$
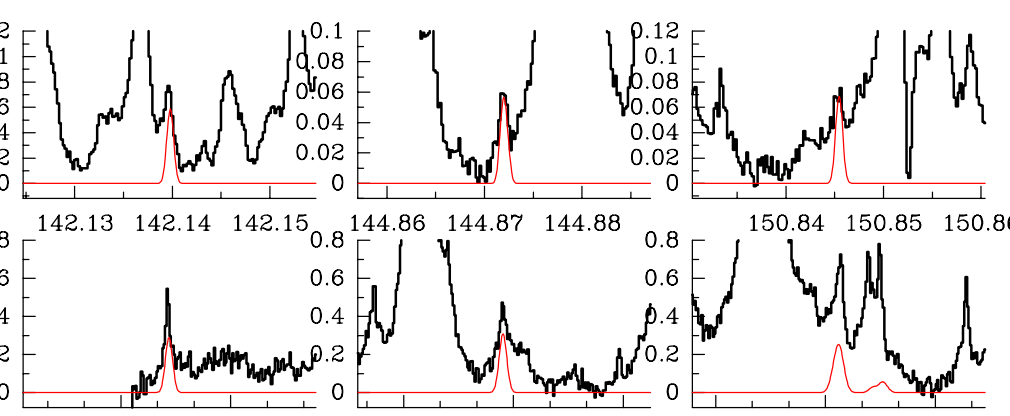

$\begin{array}{lll}144.86 & 144.87 & 144.88\end{array}$

$\begin{array}{lll}150.84 \quad 150.85 & 150.86\end{array}$
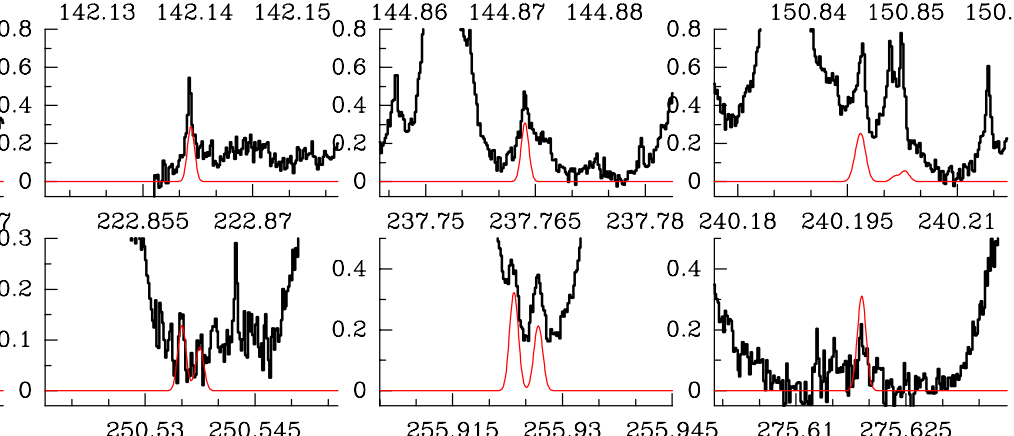

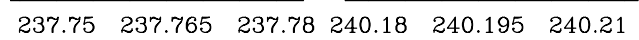
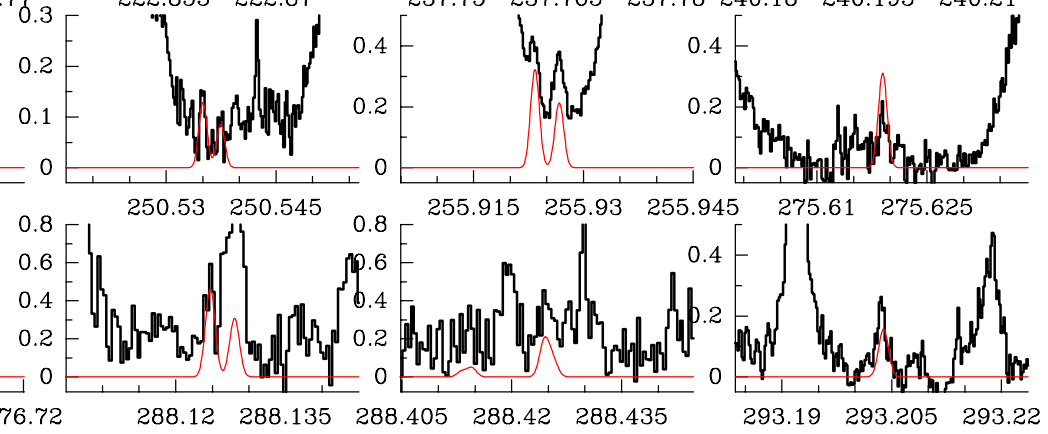

Frequency $(\mathrm{GHz})$
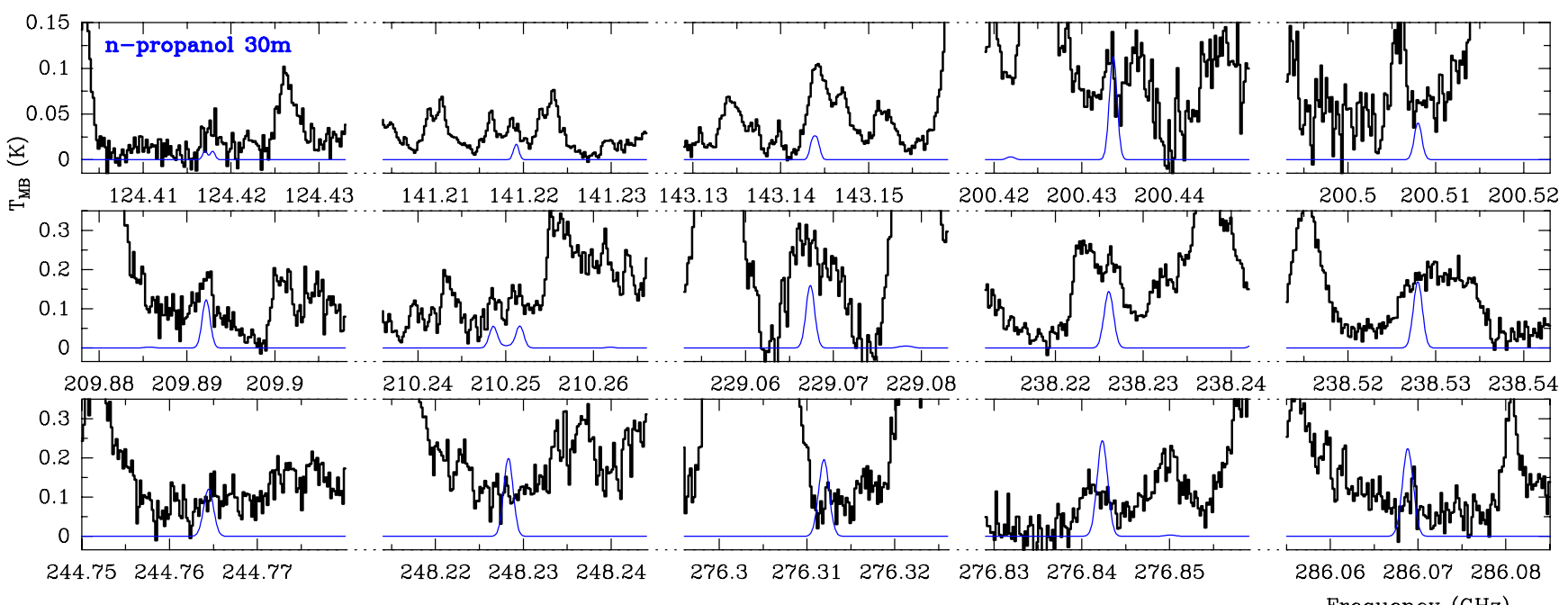

Fig. A.2. Top panel: selected lines of trans ethyl methyl ether, $\mathrm{t}-\mathrm{CH}_{3} \mathrm{CH}_{2} \mathrm{OCH}_{3}$, towards Orion $\mathrm{KL}$ detected with the IRAM $30 \mathrm{~m}$ telescope. Data in the frequency range $124-151 \mathrm{GHz}$ are those of the survey position. From 201 to $293.5 \mathrm{GHz}$ the data are those of the CR (see Sect. 2), where the emission peak of organic saturated O-rich species such as dimethyl ether $\left(\mathrm{CH}_{3} \mathrm{OCH}_{3}\right)$ and methyl formate $\left(\mathrm{CH}_{3} \mathrm{OCOH}\right)$ is located $(\mathrm{Favre}$ et al. 2011; Brouillet et al. 2013). A $v_{\mathrm{LSR}}$ of $+7.5 \mathrm{~km} \mathrm{~s}^{-1}$ is assumed. Bottom panel: selected lines of gauche-trans-n- $\mathrm{Propanol}_{1} \mathrm{Gt}_{-} \mathrm{n}-\mathrm{CH}_{3} \mathrm{CH}_{2} \mathrm{CH}_{2} \mathrm{OH}$, towards Orion KL detected with the IRAM $30 \mathrm{~m}$ telescope. A $v_{\mathrm{LSR}}$ of $+7.5 \mathrm{~km} \mathrm{~s}^{-1}$ is assumed. 
B. Tercero et al.: Trans ethyl methyl ether in space

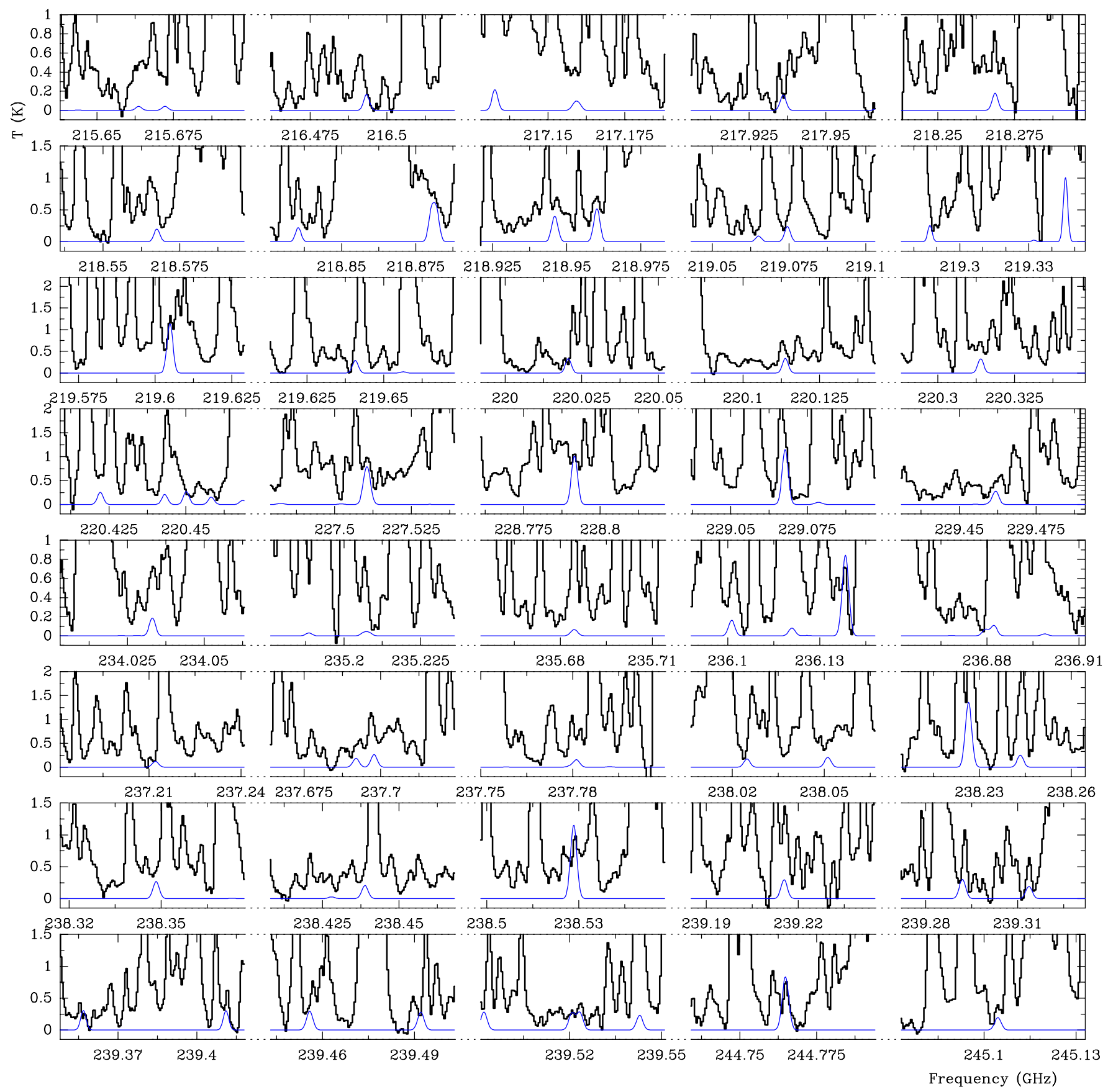

Fig. A.3. Lines of gauche-trans-n-propanol, Gt-n- $\mathrm{CH}_{3} \mathrm{CH}_{2} \mathrm{CH}_{2} \mathrm{OH}$, towards Orion KL detected with the ALMA interferometer in Position B (see text). A $v_{\mathrm{LSR}}$ of $+8 \mathrm{~km} \mathrm{~s}^{-1}$ is assumed. 
A\&A 582, L1 (2015)

Table A.1. Lines of trans- $\mathrm{CH}_{3} \mathrm{CH}_{2} \mathrm{OCH}_{3}$ in ALMA SV data.

\begin{tabular}{|c|c|c|c|c|c|c|c|}
\hline Species & $\begin{array}{c}\text { Transition } \\
J_{K_{\mathrm{a}}, K_{\mathrm{c}}}-J_{K_{\mathrm{a}}^{\prime}, K_{\mathrm{c}}^{\prime}}^{\prime}\end{array}$ & $\begin{array}{c}\text { Predicted } \\
\text { frequency }(\mathrm{MHz})\end{array}$ & $\begin{array}{r}E_{\text {upp }} \\
(\mathrm{K})\end{array}$ & $S_{i j}$ & $\begin{array}{c}\text { Observed } \\
\text { frequency }(\mathrm{MHz})\end{array}$ & $\begin{array}{c}T \\
(\mathrm{~K})\end{array}$ & Blends \\
\hline tEME-EE' & $31_{5,27}-31_{4,28}$ & 213854.674 & 220.5 & 16.34 & $\ldots$ & $\ldots$ & $\mathrm{CH}_{3} \mathrm{CH}_{2} \mathrm{OH}$ \\
\hline tEME-EE & $31_{5,27}-31_{4,28}$ & 213854.982 & 220.5 & 16.44 & $\ldots$ & $\ldots$ & " \\
\hline tEME-AE & $31_{5,27}-31_{4,28}$ & 213855.220 & 220.5 & 16.40 & $\ldots$ & $\ldots$ & $"$ \\
\hline tEME-EE & $28_{5,23}-28_{4,24}$ & 213856.708 & 185.7 & 14.17 & $\ldots$ & $\ldots$ & $"$ \\
\hline tEME-AE & $28_{5,23}-28_{4,24}$ & 213857.473 & 185.7 & 13.94 & $\ldots$ & $\ldots$ & " \\
\hline tEME-EE' & $28_{5,23}-28_{4,24}$ & 213857.509 & 185.7 & 13.69 & $\ldots$ & $\ldots$ & " \\
\hline tEME-EA & $31_{5,27}-31_{4,28}$ & 213858.239 & 220.5 & 16.52 & $\ldots$ & $\ldots$ & " \\
\hline tEME-AA & $31_{5,27}-31_{4,28}$ & 213858.632 & 220.5 & 16.53 & $\ldots$ & $\ldots$ & " \\
\hline tEME-EA & $28_{5,23}-28_{4,24}$ & 213859.015 & 185.7 & 14.70 & $\ldots$ & $\ldots$ & $"$ \\
\hline tEME-AA & $28_{5,23}-28_{4,24}$ & 213859.367 & 185.7 & 14.73 & $\ldots$ & $\ldots$ & " \\
\hline tEME-EE' & $30_{5,26}-30_{4,27}$ & 214028.729 & 208.6 & 15.58 & $\ldots$ & $\ldots$ & $\mathrm{CH}_{3} \mathrm{COCH}_{3}$ \\
\hline tEME-EE & $30_{5,26}-30_{4,27}$ & 214029.157 & 208.6 & 15.76 & $\ldots$ & $\ldots$ & " \\
\hline tEME-AE & $30_{5,26}-30_{4,27}$ & 214029.345 & 208.6 & 15.68 & $\ldots$ & $\ldots$ & " \\
\hline tEME-EA & $30_{5,26}-30_{4,27}$ & 214032.539 & 208.6 & 15.91 & $214032.3^{\dagger}$ & 0.50 & \\
\hline tEME-AA & $30_{5,26}-30_{4,27}$ & 214032.539 & 208.6 & 15.91 & $\dagger$ & & \\
\hline tEME-EE & $27_{5,22}-27_{4,23}$ & 214131.118 & 174.9 & 13.15 & 214132.6 & 0.58 & \\
\hline tEME-AE & $27_{5,22}-27_{4,23}$ & 214132.010 & 174.9 & 12.80 & $\dagger$ & & \\
\hline tEME-EE' & $27_{5,22}-27_{4,23}$ & 214132.166 & 174.9 & 12.46 & $\dagger$ & & \\
\hline tEME-EA & $27_{5,22}-27_{4,23}$ & 214133.150 & 174.9 & 14.07 & $\dagger$ & & \\
\hline tEME-AA & $27_{5,22}-27_{4,23}$ & 214133.486 & 174.9 & 14.14 & $\dagger$ & & \\
\hline tEME-EE' & $29_{5,25}-29_{4,26}$ & 214196.531 & 196.9 & 14.73 & 214196.8 & 0.94 & \\
\hline tEME-EE & $29_{5,25}-29_{4,26}$ & 214197.122 & 196.9 & 15.01 & $\dagger$ & & \\
\hline tEME-AE & $29_{5,25}-29_{4,26}$ & 214197.239 & 196.9 & 14.88 & $\dagger$ & & \\
\hline tEME-EA & $29_{5,25}-29_{4,26}$ & 214200.673 & 196.9 & 15.31 & 214201.4 & 0.41 & \\
\hline tEME-AA & $29_{5,25}-29_{4,26}$ & 214201.091 & 196.9 & 15.31 & $\dagger$ & & \\
\hline tEME-EE' & $21_{2,20}-20_{1,19}$ & 214246.202 & 93.7 & 7.76 & 214246.5 & 0.72 & \\
\hline tEME-EE & $21_{2,20}-20_{1,19}$ & 214246.202 & 93.7 & 7.76 & $\dagger$ & & \\
\hline tEME-AE & $21_{2,20}-20_{1,19}$ & 214246.332 & 93.7 & 7.76 & $\dagger$ & & \\
\hline tEME-EA & $21_{2,20}-20_{1,19}$ & 214247.602 & 93.7 & 7.76 & $\dagger$ & & \\
\hline tEME-AA & $21_{2,20}-20_{1,19}$ & 214247.732 & 93.7 & 7.76 & $\dagger$ & & \\
\hline tEME-EE' & $26_{5,22}-26_{4,22}$ & 214355.828 & 164.4 & 2.47 & $\ldots$ & $\ldots$ & $\mathrm{CH}_{3} \mathrm{CH}_{2} \mathrm{OH} ; \mathrm{SO}$ \\
\hline tEME-EE' & $28_{5,24}-28_{4,25}$ & 214356.510 & 185.7 & 13.69 & $\ldots$ & $\ldots$ & " \\
\hline tEME-AE & $26_{5,22}-26_{4,22}$ & 214356.963 & 164.4 & 2.08 & $\ldots$ & $\ldots$ & $"$ \\
\hline tEME-EE & $26_{5,22}-26_{4,22}$ & 214357.232 & 164.4 & 1.63 & $\ldots$ & $\ldots$ & $"$ \\
\hline tEME-EE & $28_{5,24}-28_{4,25}$ & 214357.312 & 185.7 & 14.17 & $\ldots$ & $\ldots$ & $"$ \\
\hline tEME-AE & $28_{5,24}-28_{4,25}$ & 214357.333 & 185.7 & 13.93 & $\ldots$ & $\ldots$ & $"$ \\
\hline tEME-EA & $28_{5,24}-28_{4,25}$ & 214361.091 & 185.7 & 14.69 & $\ldots$ & $\ldots$ & $\mathrm{CH}_{3} \mathrm{OCH}_{3}$ \\
\hline tEME-AA & $28_{5,24}-28_{4,25}$ & 214361.527 & 185.7 & 14.72 & $\ldots$ & $\ldots$ & " \\
\hline tEME-EE & $26_{5,21}-26_{4,22}$ & 214369.161 & 164.4 & 11.92 & $\ldots$ & $\ldots$ & $\mathrm{CH}_{3} \mathrm{COOCH}_{3}$ \\
\hline tEME-AE & $26_{5,21}-26_{4,22}$ & 214370.185 & 164.4 & 11.47 & $\ldots$ & $\ldots$ & " \\
\hline tEME-EE' & $26_{5,21}-26_{4,22}$ & 214370.456 & 164.4 & 11.08 & $\ldots$ & $\ldots$ & " \\
\hline tEME-EA & $26_{5,21}-26_{4,22}$ & 214370.843 & 164.4 & 13.42 & $\ldots$ & $\ldots$ & " \\
\hline tEME-AA & $26_{5,21}-26_{4,22}$ & 214371.154 & 164.4 & 13.55 & $\ldots$ & $\ldots$ & $"$ \\
\hline tEME-EE' & $27_{5,23}-27_{4,24}$ & 214507.462 & 174.9 & 12.45 & 214508.5 & 0.61 & \\
\hline tEME-AE & $27_{5,23}-27_{4,24}$ & 214508.414 & 174.9 & 12.79 & $\dagger$ & & \\
\hline tEME-EE & $27_{5,23}-27_{4,24}$ & 214508.510 & 174.9 & 13.14 & $\dagger$ & & \\
\hline tEME-EA & $27_{5,23}-27_{4,24}$ & 214512.591 & 174.9 & 14.06 & $\ldots$ & $\ldots$ & $\mathrm{CH}_{3} \mathrm{OCOH} v_{t}=1$ \\
\hline tEME-AA & $27_{5,23}-27_{4,24}$ & 214513.051 & 174.9 & 14.13 & $\ldots$ & $\ldots$ & $\prime \prime$ \\
\hline tEME-EE & $25_{5,20}-25_{4,21}$ & 214575.213 & 154.4 & 10.56 & 214576.5 & 0.58 & \\
\hline tEME-AE & $25_{5,20}-25_{4,21}$ & 214576.349 & 154.4 & 10.10 & $\dagger$ & & \\
\hline tEME-EA & $25_{5,20}-25_{4,21}$ & 214576.498 & 154.4 & 12.69 & $\dagger$ & & \\
\hline tEME-EE' & $25_{5,20}-25_{4,21}$ & 214576.706 & 154.4 & 9.74 & $\dagger$ & & \\
\hline tEME-AA & $25_{5,20}-25_{4,21}$ & 214576.769 & 154.4 & 12.96 & $\dagger$ & & \\
\hline tEME-EE' & $26_{5,22}-26_{4,23}$ & 214648.535 & 164.4 & 11.08 & $\ldots$ & $\ldots$ & $\mathrm{CH}_{3} \mathrm{OCOH}$ \\
\hline tEME-AE & $26_{5,22}-26_{4,23}$ & 214649.611 & 164.4 & 11.47 & $\ldots$ & $\ldots$ & $"$ \\
\hline tEME-EE & $26_{5,22}-26_{4,23}$ & 214649.829 & 164.4 & 11.92 & $\ldots$ & $\ldots$ & " \\
\hline tEME-EA & $26_{5,22}-26_{4,23}$ & 214654.288 & 164.5 & 13.41 & $\ldots$ & $\ldots$ & " \\
\hline tEME-AA & $26_{5,22}-26_{4,23}$ & 214654.781 & 164.5 & 13.54 & $\ldots$ & $\ldots$ & $"$ \\
\hline tEME-EE & $24_{5,19}-24_{4,20}$ & 214753.118 & 144.7 & 9.23 & 214754.0 & 0.94 & \\
\hline tEME-EA & $24_{5,19}-24_{4,20}$ & 214754.023 & 144.7 & 11.84 & $\dagger$ & & \\
\hline tEME-AA & $24_{5,19}-24_{4,20}$ & 214754.233 & 144.7 & 12.38 & $\dagger$ & & \\
\hline tEME-AE & $24_{5,19}-24_{4,20}$ & 214754.329 & 144.7 & 8.86 & $\dagger$ & & \\
\hline tEME-EE' & $24_{5,19}-24_{4,20}$ & 214754.733 & 144.7 & 8.60 & $\dagger$ & & \\
\hline tEME-EE' & $25_{5,21}-25_{4,22}$ & 214779.208 & 154.4 & 9.73 & $\ldots$ & $\ldots$ & $\mathrm{CH}_{3} \mathrm{OCOH}$ \\
\hline tEME-AE & $25_{5,21}-25_{4,22}$ & 214780.378 & 154.4 & 10.10 & $\ldots$ & $\ldots$ & $"$ \\
\hline tEME-EE & $25_{5,21}-25_{4,22}$ & 214780.701 & 154.4 & 10.56 & $\ldots$ & $\ldots$ & $"$ \\
\hline tEME-EA & $25_{5,21}-25_{4,22}$ & 214785.581 & 154.4 & 12.69 & $\ldots$ & $\ldots$ & " \\
\hline
\end{tabular}

Notes. Lines of trans- $\mathrm{CH}_{3} \mathrm{CH}_{2} \mathrm{OCH}_{3}$ (tEME) ground state present in the spectral scan of Orion KL from the ALMA interferometer. Column 1 indicates the species, Col. 2 gives the transition, Col. 3 the predicted frequency, Col. 4 upper level energy, Col. 5 the line strength, Col. 6 observed frequency at the peak channel of the line (relative to a $v_{\mathrm{LSR}}$ of $+7.5 \mathrm{~km} \mathrm{~s}^{-1}$ ), Col. 7 brightness temperature at the peak channel of the line, and Col. 8 shows blends with other molecular species. ${ }^{(\dagger)}$ Blended with previous line. 
B. Tercero et al.: Trans ethyl methyl ether in space

Table A.1. continued.

\begin{tabular}{|c|c|c|c|c|c|c|c|}
\hline Species & $\begin{array}{c}\text { Transition } \\
J_{K_{\mathrm{a}}, K_{\mathrm{c}}}-J_{K_{\mathrm{a}}^{\prime}, K_{\mathrm{c}}^{\prime}}^{\prime}\end{array}$ & $\begin{array}{c}\text { Predicted } \\
\text { frequency }(\mathrm{MHz})\end{array}$ & $\begin{array}{r}E_{\text {upp }} \\
(\mathrm{K})\end{array}$ & $S_{i j}$ & $\begin{array}{c}\text { Observed } \\
\text { frequency }(\mathrm{MHz})\end{array}$ & $\begin{array}{c}T \\
(\mathrm{~K})\end{array}$ & Blends \\
\hline tEME-AA & $25_{5,21}-25_{4,22}$ & 214786.123 & 154.4 & 12.96 & $\ldots$ & $\ldots$ & " \\
\hline tEME-EE' & $23_{5,19}-23_{4,19}$ & 214895.777 & 135.4 & 4.03 & $\ldots$ & $\ldots$ & $\mathrm{CH}_{3} \mathrm{OCOH} v_{t}=1$ \\
\hline tEME-AE & $23_{5,19}-23_{4,19}$ & 214897.184 & 135.4 & 3.90 & $\ldots$ & $\ldots$ & " \\
\hline tEME-EE & $23_{5,19}-23_{4,19}$ & 214897.739 & 135.4 & 3.67 & $\ldots$ & $\ldots$ & $"$ \\
\hline tEME-EE' & $24_{5,20}-24_{4,21}$ & 214899.228 & 144.7 & 8.61 & $\ldots$ & $\ldots$ & " \\
\hline tEME-AE & $24_{5,20}-24_{4,21}$ & 214900.454 & 144.7 & 8.87 & $\ldots$ & $\ldots$ & $"$ \\
\hline tEME-EE & $24_{5,20}-24_{4,21}$ & 214900.844 & 144.7 & 9.23 & $\ldots$ & $\ldots$ & $"$ \\
\hline tEME-EA & $23_{5,19}-23_{4,19}$ & 214903.639 & 144.7 & 1.05 & $\ldots$ & $\ldots$ & $"$ \\
\hline tEME-EA & $24_{5,20}-24_{4,21}$ & 214906.128 & 144.7 & 11.83 & 214906.7 & 1.30 & \\
\hline tEME-EE & $23_{5,18}-23_{4,19}$ & 214906.260 & 135.4 & 8.13 & $\dagger$ & & \\
\hline tEME-AA & $24_{5,20}-24_{4,21}$ & 214906.739 & 144.7 & 12.38 & $\dagger$ & & \\
\hline tEME-EA & $23_{5,18}-23_{4,19}$ & 214906.872 & 135.4 & 10.76 & $\dagger$ & & \\
\hline tEME-AA & $23_{5,18}-23_{4,19}$ & 214906.992 & 135.4 & 11.80 & $\dagger$ & & \\
\hline tEME-AE & $23_{5,18}-23_{4,19}$ & 214907.507 & 135.4 & 7.91 & $\dagger$ & & \\
\hline tEME-EE' & $23_{5,18}-23_{4,19}$ & 214907.507 & 135.4 & 7.77 & $\dagger$ & & \\
\hline tEME-EE & $24_{5,19}-24_{4,21}$ & 214909.858 & 144.7 & 11.83 & $\ldots$ & $\ldots$ & $\mathrm{CH}_{3} \mathrm{CH}_{2} \mathrm{CN}$ \\
\hline tEME-EA & $24_{5,19}-24_{4,21}$ & 214910.548 & 144.7 & 0.55 & $\ldots$ & $\ldots$ & $"$ \\
\hline tEME-AE & $24_{5,19}-24_{4,21}$ & 214911.166 & 144.7 & 3.51 & $\ldots$ & $\ldots$ & $"$ \\
\hline tEME-EE' & $24_{5,19}-24_{4,21}$ & 214911.685 & 144.7 & 3.77 & $\ldots$ & $\ldots$ & $"$ \\
\hline tEME-EE' & $23_{5,19}-23_{4,20}$ & 215008.545 & 135.4 & 7.77 & $\ldots$ & $\ldots$ & $\mathrm{CH}_{3} \mathrm{OCOH} v_{t}=2$ \\
\hline tEME-AE & $23_{5,19}-23_{4,20}$ & 215009.791 & 135.4 & 7.91 & $\ldots$ & $\ldots$ & " \\
\hline tEME-EE & $23_{5,19}-23_{4,20}$ & 215010.209 & 135.4 & 8.13 & $\ldots$ & $\ldots$ & " \\
\hline tEME-EA & $23_{5,19}-23_{4,20}$ & 215015.810 & 135.4 & 10.75 & 215016.5 & 1.16 & \\
\hline tEME-AA & $23_{5,19}-23_{4,20}$ & 215016.519 & 135.4 & 11.80 & $\dagger$ & & \\
\hline tEME-EE & $23_{5,18}-23_{4,20}$ & 215018.730 & 135.4 & 3.67 & $\ldots$ & $\ldots$ & $\mathrm{CH}_{3} \mathrm{OCOD}$ \\
\hline tEME-EA & $23_{5,18}-23_{4,20}$ & 215019.043 & 135.4 & 1.05 & $\ldots$ & $\ldots$ & " \\
\hline tEME-AE & $23_{5,18}-23_{4,20}$ & 215020.114 & 135.4 & 3.90 & $\ldots$ & $\ldots$ & " \\
\hline tEME-EE' & $23_{5,18}-23_{4,20}$ & 215020.693 & 135.4 & 4.03 & $\ldots$ & $\ldots$ & " \\
\hline tEME-EE' & $22_{5,18}-22_{4,18}$ & 215027.250 & 126.6 & 4.02 & $\ldots$ & $\ldots$ & U-line \\
\hline tEME-AE & $22_{5,18}-22_{4,18}$ & 215028.719 & 126.6 & 3.99 & $\ldots$ & $\ldots$ & $"$ \\
\hline tEME-EE & $22,18-224,18$ & 215029.330 & 126.6 & 4.00 & $\ldots$ & $\ldots$ & $"$ \\
\hline tEME-EA & $22_{5,18}-22_{4,18}$ & 215035.544 & 126.6 & 1.78 & $\ldots$ & $\ldots$ & $\mathrm{CH}_{3} \mathrm{OCOD}, \mathrm{CH}_{3} \mathrm{COOCH}_{3}$ \\
\hline tEME-EE & $22_{5,17}-22_{4,18}$ & 215037.639 & 126.6 & 7.33 & $\ldots$ & $\ldots$ & " \\
\hline tEME-EA & $22_{5,17}-22_{4,18}$ & 215038.085 & 126.6 & 9.45 & $\ldots$ & $\ldots$ & $"$ \\
\hline tEME-AA & $22_{5,17}-22_{4,18}$ & 215038.089 & 126.6 & 11.23 & $\ldots$ & $\ldots$ & $\mathrm{CH}_{3} \mathrm{CH}_{2} \mathrm{CN}$ \\
\hline tEME-AE & $225,17-224,18$ & 215038.890 & 126.6 & 7.24 & $\ldots$ & $\ldots$ & " \\
\hline tEME-EE' & $22_{5,17}-22_{4,18}$ & 215039.292 & 126.6 & 7.21 & $\ldots$ & $\ldots$ & " \\
\hline tEME-AA & $35_{2,33}-34_{3,32}$ & 215107.148 & 250.8 & 9.27 & $\ldots$ & $\ldots$ & $\mathrm{CH}_{3} \mathrm{COOCH}_{3}, \mathrm{CH}_{3} \mathrm{OCOH} v_{t}=2$ \\
\hline tEME-EA & $35_{2,33}-34_{3,32}$ & 215107.270 & 250.8 & 9.27 & $\ldots$ & $\cdots$ & " \\
\hline tEME-EE' & $22_{5,18}-22_{4,19}$ & 215107.270 & 126.6 & 7.21 & $\ldots$ & $\ldots$ & " \\
\hline tEME-AE & $22_{5,18}-22_{4,19}$ & 215108.508 & 126.6 & 7.24 & $\ldots$ & $\ldots$ & $"$ \\
\hline tEME-EE & $22_{5,18}-22_{4,19}$ & 215108.923 & 126.6 & 7.33 & $\ldots$ & $\ldots$ & $"$ \\
\hline tEME-AE & $35_{2,33}-34_{3,32}$ & 215109.184 & 250.8 & 9.27 & $\ldots$ & $\ldots$ & $"$ \\
\hline tEME-EE & $35_{2,33}-34_{3,32}$ & 215109.306 & 250.8 & 9.27 & $\ldots$ & $\ldots$ & $"$ \\
\hline tEME-EE' & $35_{2,33}-34_{3,32}$ & 215109.306 & 250.8 & 9.27 & $\ldots$ & $\ldots$ & $"$ \\
\hline tEME-EA & $22_{5,18}-22_{4,19}$ & 215114.713 & 126.6 & 9.44 & $\cdots$ & $\begin{array}{l}\cdots \\
\ldots\end{array}$ & $\mathrm{CH}_{3} \mathrm{O}^{13} \mathrm{COH}$ \\
\hline tEME-AA & $225,18-224,19$ & 215115.545 & 126.6 & 11.22 & $\ldots$ & $\ldots$ & " \\
\hline tEME-EE & $22_{5,17}-22_{4,19}$ & 215117.232 & 126.6 & 3.90 & $\ldots$ & $\ldots$ & $\mathrm{CH}_{3} \mathrm{CH}_{2} \mathrm{CN}$ \\
\hline tEME-EA & $22_{5,17}-22_{4,19}$ & 215117.254 & 126.6 & 1.78 & $\ldots$ & $\ldots$ & " \\
\hline tEME-AE & $22_{5,17}-22_{4,19}$ & 215118.679 & 126.6 & 3.99 & $\ldots$ & $\ldots$ & $"$ \\
\hline tEME-EE' & $22_{5,17}-22_{4,19}$ & 215119.312 & 126.6 & 4.02 & $\ldots$ & $\ldots$ & $"$ \\
\hline tEME-EE' & $21_{5,17}-21_{4,17}$ & 215139.474 & 118.0 & 3.75 & $\ldots$ & $\ldots$ & $\mathrm{CH}_{3} \mathrm{O}^{13} \mathrm{COH} v_{t}=1$ \\
\hline tEME-AE & $21_{5,17}-21_{4,17}$ & 215141.013 & 118.0 & 3.82 & 215141.0 & 0.59 & $\mathrm{CH}_{3} \mathrm{O}^{13} \mathrm{COH} v_{t}=1$ \\
\hline tEME-EE & $21_{5,17}-21_{4,17}$ & 215141.682 & 118.0 & 3.85 & $\dagger$ & & \\
\hline tEME-EA & $21_{5,17}-21_{4,17}$ & 215148.139 & 118.0 & 2.58 & 215150.7 & 1.28 & \\
\hline tEME-EE & $21_{5,16}-21_{4,17}$ & 215149.914 & 118.0 & 6.81 & $\dagger$ & & \\
\hline tEME-AA & $21_{5,16}-21_{4,17}$ & 215150.202 & 118.0 & 10.66 & $\dagger$ & & \\
\hline tEME-EA & $21_{5,16}-21_{4,17}$ & 215150.327 & 118.0 & 8.07 & $\dagger$ & & \\
\hline tEME-AE & $21_{5,16}-21_{4,17}$ & 215151.142 & 118.0 & 6.84 & $215150.7 \dagger$ & 1.28 & \\
\hline tEME-EE' & $21_{5,16}-21_{4,17}$ & 215151.503 & 118.0 & 6.91 & $\dagger$ & & \\
\hline tEME-EE' & $22_{5,17}-22_{4,18}$ & 215195.665 & 118.0 & 6.91 & $\ldots$ & $\ldots$ & SO \\
\hline tEME-AE & $22_{5,17}-22_{4,18}$ & 215196.869 & 118.0 & 6.84 & $\ldots$ & $\ldots$ & $"$ \\
\hline tEME-EE & $22_{5,17}-22_{4,18}$ & 215197.254 & 118.0 & 6.81 & $\ldots$ & $\ldots$ & $"$ \\
\hline tEME-EA & $22,17-22_{4,18}$ & 215203.100 & 118.0 & 8.07 & $\ldots$ & $\ldots$ & $"$ \\
\hline tEME-AA & $22_{5,17}-22_{4,18}$ & 215204.068 & 118.0 & 10.66 & $\ldots$ & $\cdots$ & " \\
\hline tEME-EA & $22_{5,16}-22_{4,18}$ & 215205.287 & 118.0 & 2.58 & $\ldots$ & $\ldots$ & $"$ \\
\hline tEME-EE & $22_{5,16}-22_{4,18}$ & 215205.486 & 118.0 & 3.84 & $\ldots$ & $\ldots$ & $"$ \\
\hline tEME-AE & $22_{5,16}-22_{4,18}$ & 215206.998 & 118.0 & 3.82 & $\ldots$ & $\ldots$ & $"$ \\
\hline tEME-EE' & $22_{5,16}-224,18$ & 215207.694 & 118.0 & 3.75 & $\ldots$ & $\begin{array}{l}\cdots \\
\ldots\end{array}$ & $"$ \\
\hline tEME-EE' & $20_{5,16}-20_{4,16}$ & 215234.851 & 109.9 & 3.26 & $\ldots$ & $\ldots$ & $"$ \\
\hline tEME-AE & $20_{5,16}-20_{4,16}$ & 215236.480 & 109.9 & 3.41 & $\ldots$ & $\ldots$ & $"$ \\
\hline tEME-EE & $20_{5,16}-20_{4,16}$ & 215237.223 & 109.9 & 3.55 & $\ldots$ & $\ldots$ & $" \prime$ \\
\hline tEME-EA & $20_{5,16}-20_{4,16}$ & 215243.901 & 109.9 & 3.19 & $\ldots$ & $\ldots$ & $\mathrm{CH}_{3} \mathrm{COOCH}_{3}$ \\
\hline
\end{tabular}


A\&A 582, L1 (2015)

Table A.1. continued.

\begin{tabular}{|c|c|c|c|c|c|c|c|}
\hline Species & $\begin{array}{c}\text { Transition } \\
J_{K_{\mathrm{a}}, K_{\mathrm{c}}}-J_{K_{\mathrm{a}}^{\prime}, K_{\mathrm{c}}^{\prime}}^{\prime}\end{array}$ & $\begin{array}{c}\text { Predicted } \\
\text { frequency }(\mathrm{MHz})\end{array}$ & $\begin{array}{r}E_{\text {upp }} \\
(\mathrm{K})\end{array}$ & $S_{i j}$ & $\begin{array}{c}\text { Observed } \\
\text { frequency }(\mathrm{MHz})\end{array}$ & $\begin{array}{c}T \\
(\mathrm{~K})\end{array}$ & Blends \\
\hline & & & & & & & $\mathrm{CH}_{3} \mathrm{O}^{13} \mathrm{COH}, \mathrm{CH}_{3} \mathrm{CH}_{2} \mathrm{CN} v_{13} / v_{21}$ \\
\hline tEME-EE & $20_{5,15}-20_{4,16}$ & 215245.438 & 109.9 & 6.54 & $\cdots$ & $\ldots$ & \\
\hline tEME-AA & $20_{5,15}-20_{4,16}$ & 215245.691 & 109.9 & 10.09 & $\ldots$ & $\ldots$ & " \\
\hline tEME-EA & $20_{5,15}-20_{4,16}$ & 215245.934 & 109.9 & 6.89 & $\ldots$ & $\ldots$ & $"$ \\
\hline tEME-AE & $20_{5,15}-20_{4,16}$ & 215246.615 & 109.9 & 6.67 & $\ldots$ & $\ldots$ & $"$ \\
\hline tEME-EE' & $20_{5,15}-20_{4,16}$ & 215246.907 & 109.9 & 6.83 & $\ldots$ & $\ldots$ & $"$ \\
\hline tEME-EE' & $20_{5,16}-20_{4,17}$ & 215274.126 & 109.9 & 6.83 & $\ldots$ & $\ldots$ & $\mathrm{CH}_{3} \mathrm{COOCH}_{3}$, U-line \\
\hline tEME-AE & $20_{5,16}-20_{4,17}$ & 215275.268 & 109.9 & 6.67 & $\ldots$ & $\ldots$ & " \\
\hline tEME-EE & $20_{5,16}-20_{4,17}$ & 215275.595 & 109.9 & 6.54 & $\ldots$ & $\ldots$ & $"$ \\
\hline tEME-EA & $20_{5,16}-20_{4,17}$ & 215281.378 & 109.9 & 6.89 & $\ldots$ & $\ldots$ & U-line \\
\hline tEME-AA & $20_{5,16}-20_{4,17}$ & 215282.470 & 109.9 & 10.09 & $\ldots$ & $\ldots$ & " \\
\hline tEME-EA & $20_{5,15}-20_{4,17}$ & 215283.411 & 109.9 & 3.19 & $\ldots$ & $\ldots$ & " \\
\hline tEME-EE & $20_{5,15}-20_{4,17}$ & 215283.810 & 109.9 & 3.55 & $\ldots$ & $\ldots$ & $"$ \\
\hline tEME-AE & $20_{5,15}-20_{4,17}$ & 215285.403 & 109.9 & 3.41 & $\ldots$ & $\ldots$ & $"$ \\
\hline tEME-EE' & $20_{5,15}-20_{4,17}$ & 215286.182 & 109.9 & 3.26 & $\ldots$ & $\ldots$ & $"$ \\
\hline tEME-EE' & $19_{5,15}-19_{4,15}$ & 215315.464 & 102.2 & 2.57 & $\ldots$ & $\ldots$ & U-line \\
\hline tEME-AE & $19_{5,15}-19_{4,15}$ & 215317.213 & 102.2 & 2.79 & 215317.4 & 0.54 & U-line \\
\hline tEME-EE & $19_{5,15}-19_{4,15}$ & 215318.057 & 102.2 & 3.02 & + & & \\
\hline tEME-EA & $19_{5,15}-19_{4,15}$ & 215324.992 & 102.2 & 3.49 & 215327.2 & 1.96 & ${ }^{13} \mathrm{CH}_{3} \mathrm{OCOH} v_{t}=1$ \\
\hline tEME-EE & $19_{5,14}-19_{4,15}$ & 215326.280 & 102.2 & 6.50 & $\dagger$ & & \\
\hline tEME-AA & $19_{5,14}-19_{4,15}$ & 215326.637 & 102.2 & 9.52 & $\dagger$ & & \\
\hline tEME-EA & $19_{5,14}-19_{4,15}$ & 215326.971 & 102.2 & 6.03 & $\dagger$ & & \\
\hline tEME-AE & $19_{5,14}-19_{4,15}$ & 215327.373 & 102.2 & 6.72 & $\dagger$ & & \\
\hline tEME-EE' & $19_{5,14}-19_{4,15}$ & 215327.561 & 102.2 & 6.95 & $\dagger$ & & \\
\hline tEME-EE' & $19_{5,15}-19_{4,16}$ & 215343.169 & 102.2 & 6.95 & $\ldots$ & $\ldots$ & $\mathrm{CH}_{3} \mathrm{OCOH}$ \\
\hline tEME-AE & $19_{5,15}-19_{4,16}$ & 215344.214 & 102.2 & 6.72 & $\ldots$ & $\ldots$ & " \\
\hline tEME-EE & $19_{5,15}-19_{4,16}$ & 215344.450 & 102.2 & 6.50 & $\ldots$ & $\ldots$. & $"$ \\
\hline tEME-EA & $19_{5,15}-19_{4,16}$ & 215350.059 & 102.2 & 6.03 & 215351.2 & 0.91 & $\mathrm{CH}_{3} \mathrm{CH}_{2} \mathrm{CN}\left(v=0, v_{13} / v_{21}\right)$ \\
\hline tEME-AA & $19_{5,15}-19_{4,16}$ & 215351.248 & 102.2 & 9.52 & $\dagger$ & & \\
\hline tEME-EA & $19_{5,14}-19_{4,16}$ & 215352.037 & 102.2 & 3.49 & $\dagger$ & & \\
\hline tEME-EE & $19_{5,14}-19_{4,16}$ & 215352.674 & 102.2 & 3.02 & $\dagger$ & & \\
\hline tEME-AE & $19_{5,14}-19_{4,16}$ & 215354.374 & 102.2 & 2.79 & $\ldots$ & $\ldots$ & $\mathrm{CH}_{3} \mathrm{CH}_{2} \mathrm{CN}\left(v=0, v_{13} / v_{21}\right)$ \\
\hline tEME-EE' & $19_{5,14}-19_{4,16}$ & 215355.266 & 102.2 & 2.57 & $\ldots$ & $\ldots$ & " \\
\hline tEME-EE' & $12_{3,10}-11_{2,9}$ & 215361.252 & 40.5 & 4.16 & 215361.7 & 0.43 & \\
\hline tEME-EE & $12_{3,10}-11_{2,9}$ & 215361.484 & 40.5 & 4.18 & $\dagger$ & & \\
\hline tEME-AE & $12_{3,10}-11_{2,9}$ & 215361.676 & 40.5 & 4.17 & $\dagger$ & & \\
\hline tEME-EA & $12_{3,10}-11_{2,9}$ & 215364.224 & 40.5 & 4.19 & $\ldots$ & $\ldots$ & $\mathrm{CH}_{3} \mathrm{CH}_{2} \mathrm{OH}$ \\
\hline tEME-AA & $12_{3,10}-11_{2,9}$ & 215364.533 & 40.5 & 4.20 & $\ldots$ & $\ldots$ & " \\
\hline tEME-EA & $18_{5,14}-18_{4,14}$ & 215393.299 & 94.8 & 3.48 & 215395.5 & 1.01 & \\
\hline tEME-EE & $18_{5,13}-18_{4,14}$ & 215394.256 & 94.8 & 6.65 & $\dagger$ & & \\
\hline tEME-AA & $18_{5,13}-18_{4,14}$ & 215394.875 & 94.8 & 8.95 & $\dagger$ & & \\
\hline tEME-AE & $18_{5,13}-18_{4,14}$ & 215395.227 & 94.8 & 6.94 & $\dagger$ & & \\
\hline tEME-EA & $18_{5,13}-18_{4,14}$ & 215395.264 & 94.8 & 5.47 & $\dagger$ & & \\
\hline tEME-EE' & $18_{5,13}-18_{4,14}$ & 215395.280 & 94.8 & 7.19 & $\dagger$ & & \\
\hline tEME-EE' & $18_{5,14}-18_{4,15}$ & 215403.394 & 94.8 & 7.19 & 215404.5 & 0.91 & \\
\hline tEME-AE & $18_{5,14}-18_{4,15}$ & 215404.308 & 94.8 & 6.94 & $\dagger$ & & \\
\hline tEME-EE & $18_{5,14}-18_{4,15}$ & 215404.417 & 94.8 & 6.65 & $\dagger$ & & \\
\hline tEME-EA & $18_{5,14}-18_{4,15}$ & 215409.728 & 94.8 & 5.47 & 215411.2 & 0.59 & \\
\hline tEME-AA & $18_{5,14}-18_{4,15}$ & 215410.979 & 94.8 & 8.95 & $\dagger$ & & \\
\hline tEME-EA & $185,14-18_{4,15}$ & 215411.693 & 94.8 & 3.48 & $\dagger$ & & \\
\hline tEME-EE & $18_{5,14}-18_{4,15}$ & 215412.657 & 94.8 & 2.31 & $\dagger$ & & \\
\hline tEME-EA & $17_{5,13}-17_{4,13}$ & 215450.467 & 87.9 & 3.22 & 215451.7 & 1.65 & \\
\hline tEME-EE & $17_{5,12}-17_{4,13}$ & 215450.974 & 87.9 & 6.90 & $\dagger$ & & \\
\hline tEME-EE' & $17_{5,12}-17_{4,13}$ & 215451.703 & 87.9 & 7.39 & $\dagger$ & & \\
\hline tEME-AE & $17_{5,12}-17_{4,13}$ & 215451.799 & 87.9 & 7.18 & $\dagger$ & & \\
\hline tEME-AA & $17_{5,12}-17_{4,13}$ & 215452.025 & 87.9 & 8.39 & $\dagger$ & & \\
\hline tEME-EA & $17_{5,12}-17_{4,13}$ & 215452.436 & 87.9 & 5.17 & $\dagger$ & & \\
\hline tEME-EE' & $17_{5,13}-17_{4,13}$ & 215455.422 & 87.9 & 7.39 & 215456.2 & 1.60 & ${ }^{13} \mathrm{CH}_{3} \mathrm{OCOH} v_{t}=1$ \\
\hline tEME-EE & $17_{5,13}-17_{4,13}$ & 215456.151 & 87.9 & 6.90 & $\dagger$ & & \\
\hline tEME-AE & $17_{5,13}-17_{4,13}$ & 215456.193 & 87.9 & 7.18 & $\dagger$ & & \\
\hline tEME-EA & $17_{5,13}-17_{4,14}$ & 215461.025 & 87.9 & 5.17 & 215462.2 & 1.81 & U-line \\
\hline tEME-AA & $17_{5,13}-17_{4,14}$ & 215462.304 & 87.9 & 8.39 & $\dagger$ & & \\
\hline tEME-EA & $17_{5,12}-17_{4,14}$ & 215462.994 & 87.9 & 3.22 & $\dagger$ & & \\
\hline tEME-EE' & $27_{1,27}-26_{0,26}$ & 215478.840 & 144.2 & 21.60 & 215479.5 & 1.81 & \\
\hline tEME-EE & $27_{1,27}-26_{0,26}$ & 215478.840 & 144.2 & 21.60 & $\dagger$ & & \\
\hline tEME-AE & $27_{1,27}-26_{0,26}$ & 215478.854 & 144.2 & 21.60 & $\dagger$ & & \\
\hline tEME-EA & $27_{1,27}-26_{0,26}$ & 215479.025 & 144.2 & 21.60 & $\dagger$ & & \\
\hline tEME-AA & $27_{1,27}-26_{0,26}$ & 215479.039 & 144.2 & 21.60 & $\dagger$ & & \\
\hline tEME-EE & $16_{5,11}-16_{4,12}$ & 215497.897 & 81.3 & 7.05 & 215498.7 & 1.96 & $\mathrm{CH}_{3} \mathrm{CH}_{2} \mathrm{CN} v_{13} / v_{21}$ \\
\hline tEME-EA & $16_{5,12}-16_{4,12}$ & 215497.943 & 81.3 & 2.74 & $\dagger$ & & \\
\hline tEME-EE' & $16_{5,11}-16_{4,12}$ & 215498.372 & 81.3 & 7.37 & $\dagger$ & & \\
\hline tEME-AE & $16_{5,11}-16_{4,12}$ & 215498.590 & 81.3 & 7.23 & $\dagger$ & & \\
\hline tEME-AA & $16_{5,11}-16_{4,12}$ & 215499.524 & 81.3 & 7.82 & $215500.2^{\dagger}$ & 1.22 & \\
\hline
\end{tabular}

L1, page 10 of 43 
B. Tercero et al.: Trans ethyl methyl ether in space

Table A.1. continued.

\begin{tabular}{|c|c|c|c|c|c|c|c|}
\hline Species & $\begin{array}{c}\text { Transition } \\
J_{K_{\mathrm{a}}, K_{\mathrm{c}}}-J_{K_{\mathrm{a}}^{\prime}, K_{\mathrm{c}}^{\prime}}^{\prime}\end{array}$ & $\begin{array}{c}\text { Predicted } \\
\text { frequency }(\mathrm{MHz})\end{array}$ & $\begin{array}{r}E_{\text {upp }} \\
(\mathrm{K})\end{array}$ & $S_{i j}$ & $\begin{array}{c}\text { Observed } \\
\text { frequency }(\mathrm{MHz})\end{array}$ & $\begin{array}{c}T \\
(\mathrm{~K})\end{array}$ & Blends \\
\hline tEME-EE' & $16_{5,12}-16_{4,13}$ & 215499.830 & 81.3 & 7.37 & ${ }^{\dagger}$ & & \\
\hline tEME-EA & $16_{5,11}-16_{4,12}$ & 215499.921 & 81.3 & 5.08 & $\dagger$ & & \\
\hline tEME-EE & $16_{5,12}-16_{4,13}$ & 215500.305 & 81.3 & 7.05 & $\dagger$ & & \\
\hline tEME-AE & $16_{5,12}-16_{4,13}$ & 215500.485 & 81.3 & 7.24 & $\dagger$ & & \\
\hline tEME-EA & $16_{5,12}-16_{4,13}$ & 215504.636 & 81.3 & 5.08 & 215506.2 & 1.22 & $\mathrm{CH}_{3} \mathrm{C}^{13} \mathrm{CH}$ \\
\hline tEME-AA & $16_{5,12}-16_{4,13}$ & 215505.905 & 81.3 & 7.82 & $\dagger$ & & \\
\hline tEME-EA & $16_{5,11}-16_{4,13}$ & 215506.613 & 81.3 & 2.74 & $\dagger$ & & \\
\hline tEME-EE & $15_{5,10}-15_{4,11}$ & 215536.404 & 75.1 & 6.94 & 215537.7 & 1.33 & \\
\hline tEME-EE' & $15_{5,10}-15_{4,11}$ & 215536.720 & 75.1 & 7.08 & $\dagger$ & & \\
\hline tEME-EA & $15_{5,11}-15_{4,11}$ & 215537.002 & 75.1 & 2.05 & $\dagger$ & & \\
\hline tEME-AE & $15_{5,10}-15_{4,11}$ & 215537.010 & 75.1 & 7.03 & $\dagger$ & & \\
\hline tEME-EE' & $15_{5,11}-15_{4,12}$ & 215537.169 & 75.1 & 7.08 & $\dagger$ & & \\
\hline tEME-EE & $15_{5,11}-15_{4,12}$ & 215537.485 & 75.1 & 6.94 & $\dagger$ & & \\
\hline tEME-AE & $15_{5,11}-15_{4,12}$ & 215537.757 & 75.1 & 7.08 & $\dagger$ & & \\
\hline tEME-AA & $15_{5,10}-15_{4,11}$ & 215538.650 & 75.1 & 7.25 & $\dagger$ & & \\
\hline tEME-EA & $15_{5,10}-15_{4,11}$ & 215538.990 & 75.1 & 5.21 & $\dagger$ & & \\
\hline tEME-EA & $15_{5,11}-15_{4,12}$ & 215541.270 & 75.1 & 5.21 & $215541.5^{\dagger}$ & 1.06 & $\mathrm{CH}_{3} \mathrm{COOCH}_{3}$ \\
\hline tEME-AA & $15_{5,11}-15_{4,12}$ & 215542.488 & 75.1 & 7.25 & $\dagger$ & & \\
\hline tEME-EA & $15_{5,10}-15_{4,12}$ & 215543.258 & 75.1 & 2.05 & $\dagger$ & & \\
\hline tEME-EE & $14_{5,9}-14_{4,10}$ & 215567.750 & 69.3 & 6.58 & 215567.9 & 2.13 & \\
\hline tEME-EE' & $14_{5,9}-14_{4,10}$ & 215567.993 & 69.3 & 6.63 & $\dagger$ & & \\
\hline tEME-EE' & $14_{5,10}-14_{4,11}$ & 215568.043 & 69.3 & 6.63 & $\dagger$ & & \\
\hline tEME-EE & $14_{5,10}-14_{4,11}$ & 215568.286 & 69.3 & 6.58 & $\dagger$ & & \\
\hline tEME-AE & $14_{5,9}-14_{4,10}$ & 215568.316 & 69.3 & 6.61 & $\dagger$ & & \\
\hline tEME-AE & $14_{5,10}-14_{4,11}$ & 215568.602 & 69.3 & 6.61 & 215571.7 & 1.89 & $\mathrm{CH}_{3} \mathrm{OCOH} v_{t}=1$ \\
\hline tEME-AE & $14_{5,10}-14_{4,10}$ & 215568.774 & 69.3 & 1.23 & $\dagger$ & & \\
\hline tEME-AA & $14_{5,9}-14_{4,10}$ & 215570.539 & 69.3 & 6.68 & $\dagger$ & & \\
\hline tEME-EA & $14_{5,9}-14_{4,10}$ & 215570.772 & 69.3 & 5.45 & $\dagger$ & & \\
\hline tEME-EA & $14_{5,10}-14_{4,11}$ & 215571.650 & 69.3 & 5.45 & $\dagger$ & & \\
\hline tEME-AA & $14_{5,10}-14_{4,11}$ & 215572.766 & 69.3 & 6.68 & $\dagger$ & & \\
\hline tEME-EA & $14_{5,9}-14_{4,11}$ & 215573.648 & 69.3 & 1.23 & $\dagger$ & & \\
\hline tEME-EE & $13_{5,8}-13_{4,9}$ & 215593.014 & 63.9 & 6.08 & 215593.5 & 1.41 & \\
\hline tEME-EE' & $13_{5,9}-13_{4,10}$ & 215593.138 & 63.9 & 6.09 & $\dagger$ & & \\
\hline tEME-EE' & $13_{5,8}-13_{4,9}$ & 215593.228 & 63.9 & 6.09 & $\dagger$ & & \\
\hline tEME-EE & $13_{5,9}-13_{4,10}$ & 215593.353 & 63.9 & 6.08 & $\dagger$ & & \\
\hline tEME-AE & $13_{5,8}-13_{4,9}$ & 215593.566 & 63.9 & 6.09 & $\dagger$ & & \\
\hline tEME-AE & $13_{5,9}-13_{4,10}$ & 215593.687 & 63.9 & 6.09 & $\dagger$ & & \\
\hline tEME-EA & $13_{5,9}-13_{4,9}$ & 215594.282 & 63.9 & 0.53 & $\dagger$ & & \\
\hline tEME-AA & $13_{5,8}-13_{4,9}$ & 215596.207 & 63.9 & 6.11 & 215596.5 & 1.12 & \\
\hline tEME-EA & $13_{5,8}-13_{4,9}$ & 215596.289 & 63.9 & 5.58 & $\dagger$ & & \\
\hline tEME-EA & $13_{5,9}-13_{4,10}$ & 215596.478 & 63.9 & 5.58 & $\dagger$ & & \\
\hline tEME-AA & $13_{5,9}-13_{4,10}$ & 215597.447 & 63.9 & 6.11 & $\dagger$ & & \\
\hline tEME-EA & $13_{5,8}-13_{4,10}$ & 215598.486 & 63.9 & 0.53 & $\dagger$ & & \\
\hline tEME-EE & $12_{5,7}-12_{4,8}$ & 215613.102 & 58.9 & 5.52 & 215613.7 & 1.89 & \\
\hline tEME-EE' & $12_{5,8}-12_{4,9}$ & 215613.172 & 58.9 & 5.53 & $\dagger$ & & \\
\hline tEME-EE' & $12_{5,7}-12_{4,8}$ & 215613.308 & 58.9 & 5.53 & $\dagger$ & & \\
\hline tEME-EE & $12_{5,8}-12_{4,9}$ & 215613.378 & 58.9 & 5.52 & $\dagger$ & & \\
\hline tEME-AE & $12_{5,7}-12_{4,8}$ & 215613.651 & 58.9 & 5.52 & $\dagger$ & & \\
\hline tEME-AE & $12_{5,8}-12_{4,9}$ & 215613.720 & 58.9 & 5.52 & $\dagger$ & & \\
\hline tEME-EA & $12_{5,8}-12_{4,9}$ & 215616.405 & 58.9 & 5.37 & $\ldots$ & $\ldots$ & ${ }^{13} \mathrm{CH}_{3} \mathrm{OCOH} v_{t}=1, \mathrm{CH}_{3} \mathrm{CH}_{2} \mathrm{CN}$ \\
\hline tEME-EA & $12_{5,7}-12_{4,8}$ & 215616.490 & 58.9 & 5.37 & $\ldots$ & $\ldots$ & " \\
\hline tEME-AA & $12_{5,7}-12_{4,8}$ & 215616.564 & 58.9 & 5.53 & $\ldots$ & $\ldots$ & $"$ \\
\hline tEME-AA & $12_{5,8}-12_{4,9}$ & 215617.221 & 58.9 & 5.53 & $\ldots$ & $\ldots$ & $"$ \\
\hline tEME-EE & $11_{5,6}-11_{4,7}$ & 215628.800 & 54.3 & 4.94 & 215629.7 & 1.60 & \\
\hline tEME-EE' & $11_{5,7}-11_{4,8}$ & 215628.855 & 54.3 & 4.94 & $\dagger$ & & \\
\hline tEME-EE' & $11_{5,6}-11_{4,7}$ & 215629.003 & 54.3 & 4.94 & $\dagger$ & & \\
\hline tEME-EE & $11_{5,7}-11_{4,8}$ & 215629.058 & 54.3 & 4.94 & $\dagger$ & & \\
\hline tEME-AE & $11_{5,6}-11_{4,7}$ & 215629.349 & 54.3 & 4.94 & $\dagger$ & & \\
\hline tEME-AE & $11_{5,7}-11_{4,8}$ & 215629.404 & 54.3 & 4.94 & $\dagger$ & & \\
\hline tEME-EA & $11_{5,7}-11_{4,8}$ & 215632.056 & 54.3 & 4.91 & 215632.7 & 1.06 & \\
\hline tEME-EA & $11_{5,6}-11_{4,7}$ & 215632.229 & 54.3 & 4.91 & $\dagger$ & & \\
\hline tEME-AA & $11_{5,6}-11_{4,7}$ & 215632.425 & 54.3 & 4.94 & $\dagger$ & & \\
\hline tEME-AA & $11_{5,7}-11_{4,8}$ & 215632.754 & 54.3 & 4.94 & $\dagger$ & & \\
\hline tEME-EE & $10_{5,5}-10_{4,6}$ & 215640.803 & 50.0 & 4.35 & 215641.1 & 1.31 & \\
\hline tEME-EE' & $10_{5,6}-10_{4,7}$ & 215640.854 & 50.0 & 4.35 & $\dagger$ & & \\
\hline tEME-EE' & $10_{5,5}-10_{4,6}$ & 215641.007 & 50.0 & 4.35 & $\dagger$ & & \\
\hline tEME-EE & $10_{5,6}-10_{4,7}$ & 215641.058 & 50.0 & 4.35 & $\dagger$ & & \\
\hline tEME-AE & $10_{5,5}-10_{4,6}$ & 215641.354 & 50.0 & 4.35 & $\dagger$ & & \\
\hline tEME-AE & $10_{5,6}-10_{4,7}$ & 215641.405 & 50.0 & 4.35 & $\dagger$ & & \\
\hline tEME-EA & $10_{5,6}-10_{4,7}$ & 215644.051 & 50.0 & 4.34 & 215644.1 & 1.26 & ${ }^{13} \mathrm{CH}_{3} \mathrm{OCOH} v_{t}=1$ \\
\hline tEME-EA & $10_{5,5}-10_{4,6}$ & 215644.248 & 50.0 & 4.34 & $\dagger$ & & \\
\hline tEME-AA & $10_{5,5}-10_{4,6}$ & 215644.522 & 50.0 & 4.35 & $\dagger$ & & \\
\hline tEME-AA & $10_{5,6}-10_{4,7}$ & 215644.676 & 50.0 & 4.35 & $\dagger$ & & \\
\hline
\end{tabular}


A\&A 582, L1 (2015)

Table A.1. continued.

\begin{tabular}{|c|c|c|c|c|c|c|c|}
\hline Species & $\begin{array}{c}\text { Transition } \\
J_{K_{\mathrm{a}}, K_{\mathrm{c}}}-J_{K_{\mathrm{a}}^{\prime}, K_{\mathrm{c}}^{\prime}}^{\prime}\end{array}$ & $\begin{array}{c}\text { Predicted } \\
\text { frequency }(\mathrm{MHz})\end{array}$ & $\begin{array}{r}E_{\text {upp }} \\
(\mathrm{K})\end{array}$ & $S_{i j}$ & $\begin{array}{c}\text { Observed } \\
\text { frequency }(\mathrm{MHz})\end{array}$ & $\begin{array}{c}T \\
(\mathrm{~K})\end{array}$ & Blends \\
\hline tEME-EE & $99_{5,4}-9_{4,5}$ & 215649.740 & 46.1 & 3.73 & 215650.2 & 1.02 & \\
\hline tEME-EE' & $9_{5,5}-9_{4,6}$ & 215649.791 & 46.1 & 3.73 & $\dagger$ & & \\
\hline tEME-EE' & $9_{5,4}-9_{4,5}$ & 215649.944 & 46.1 & 3.73 & $\dagger$ & & \\
\hline tEME-EE & $9_{5,5}-9_{4,6}$ & 215649.995 & 46.1 & 3.73 & $\dagger$ & & \\
\hline tEME-AE & $9_{5,4}-9_{4,5}$ & 215650.292 & 46.1 & 3.73 & $\dagger$ & & \\
\hline tEME-AE & $9_{5,5}-9_{4,6}$ & 215650.343 & 46.1 & 3.73 & $\dagger$ & & \\
\hline tEME-EA & $9_{5,5}-9_{4,6}$ & 215652.990 & 46.1 & 3.73 & 215653.2 & 0.92 & \\
\hline tEME-EA & $9_{5,4}-9_{4,5}$ & 215653.193 & 46.1 & 3.73 & $\dagger$ & & \\
\hline tEME-AA & $95,4-94,5$ & 215653.509 & 46.1 & 3.73 & $\dagger$ & & \\
\hline tEME-AA & $9_{5,5}-9_{4,6}$ & 215653.575 & 46.1 & 3.73 & $\dagger$ & & \\
\hline tEME-EE & $85,3-8,4$ & 215656.176 & 42.7 & 3.10 & 215656.2 & 1.16 & \\
\hline tEME-EE' & $8,8,-8_{4,5}$ & 215656.227 & 42.7 & 3.10 & $\dagger$ & & \\
\hline tEME-EE' & $8_{5,3}-8_{4,4}$ & 215656.380 & 42.7 & 3.10 & $\dagger$ & & \\
\hline tEME-EE & $85,4-84,5$ & 215656.432 & 42.7 & 3.10 & $\dagger$ & & \\
\hline tEME-AE & $8,3-8_{4,4}$ & 215656.730 & 42.7 & 3.10 & $\dagger$ & & \\
\hline tEME-AE & $85,4-84,5$ & 215656.781 & 42.7 & 3.10 & $\dagger$ & & \\
\hline tEME-EA & $8,8,-8_{4,5}$ & 215659.431 & 42.7 & 3.10 & 215660.8 & 1.16 & \\
\hline tEME-EA & $8_{5,3}-8_{4,4}$ & 215659.635 & 42.7 & 3.10 & $\dagger$ & & \\
\hline tEME-AA & $8_{5,3}-8_{4,4}$ & 215659.972 & 42.7 & 3.10 & $\dagger$ & & \\
\hline tEME-AA & $8,8,-84,5$ & 215659.998 & 42.7 & 3.10 & $\dagger$ & & \\
\hline tEME-EE & $7_{5,2}-7_{4,3}$ & 215660.620 & 39.6 & 2.43 & $\dagger$ & & \\
\hline tEME-EE' & $7_{5,3}-7_{4,4}$ & 215660.672 & 39.6 & 2.43 & $\dagger$ & & \\
\hline tEME-EE' & $7_{5,2}-7_{4,3}$ & 215660.825 & 39.6 & 2.43 & $\dagger$ & & \\
\hline tEME-EE & $7_{5,3}-7_{4,4}$ & 215660.877 & 39.6 & 2.43 & $\dagger$ & & \\
\hline tEME-AE & $7_{5,2}-7_{4,3}$ & 215661.175 & 39.6 & 2.43 & $\dagger$ & & \\
\hline tEME-AE & $7_{5,3}-7_{4,4}$ & 215661.227 & 39.6 & 2.43 & $\dagger$ & & \\
\hline tEME-EE & $6_{5,1}-6_{4,2}$ & 215663.523 & 36.9 & 1.72 & 215664.6 & 1.11 & \\
\hline tEME-EE' & $6_{5,2}-6_{4,3}$ & 215663.575 & 36.9 & 1.72 & $\dagger$ & & \\
\hline tEME-EE' & $6_{5,1}-6_{4,2}$ & 215663.728 & 36.9 & 1.72 & $\dagger$ & & \\
\hline tEME-EE & $6,2-64,3$ & 215663.780 & 36.9 & 1.72 & $\dagger$ & & \\
\hline tEME-EA & $7_{5,3}-7_{4,4}$ & 215663.879 & 39.6 & 2.43 & $\dagger$ & & \\
\hline tEME-AE & $6,6_{5,1}-6_{4,2}$ & 215664.080 & 36.9 & 1.72 & $\dagger$ & & \\
\hline tEME-EA & $7_{5,2}-7_{4,3}$ & 215664.084 & 39.6 & 2.43 & $\dagger$ & & \\
\hline tEME-AE & $6,6-6,6$ & 215664.131 & 36.9 & 1.72 & $\dagger$ & & \\
\hline tEME-AA & $7_{5,2}-7_{4,3}$ & 215664.430 & 39.6 & 2.43 & $\dagger$ & & \\
\hline tEME-AA & $75,3-7,4$ & 215664.439 & 39.6 & 2.43 & $\dagger$ & & \\
\hline tEME-EE & $5_{5,0}-5_{4,1}$ & 215665.279 & 34.6 & 0.92 & $\dagger$ & & \\
\hline tEME-EE' & $5_{5,1}-5_{4,2}$ & 215665.331 & 34.6 & 0.92 & $\dagger$ & & \\
\hline tEME-EE' & $5_{5,0}-5_{4,1}$ & 215665.485 & 34.6 & 0.92 & $\dagger$ & & \\
\hline tEME-EE & $5_{5,1}-5_{4,2}$ & 215665.537 & 34.6 & 0.92 & $\dagger$ & & \\
\hline tEME-AE & $5_{5,0}-5_{4,1}$ & 215665.837 & 34.6 & 0.92 & $\dagger$ & & \\
\hline tEME-AE & $5_{5,1}-5_{4,2}$ & 215665.889 & 34.6 & 0.92 & $\dagger$ & & \\
\hline tEME-EA & $6_{5,2}-6_{4,3}$ & 215666.786 & 36.9 & 1.72 & 215667.6 & 0.97 & $\mathrm{CH}_{3} \mathrm{CH}_{2} \mathrm{CN}$ \\
\hline tEME-AE & $6_{5,1}-6_{4,2}$ & 215666.991 & 36.9 & 1.72 & $\dagger$ & & \\
\hline tEME-AE & $6,6,-64,2$ & 215667.342 & 36.9 & 1.72 & $\dagger$ & & \\
\hline tEME-EA & $6_{5,2}-6_{4,3}$ & 215667.344 & 36.9 & 1.72 & $\dagger$ & & \\
\hline tEME-AE & $5_{5,1}-5_{4,2}$ & 215668.545 & 34.6 & 0.92 & $\dagger$ & & \\
\hline tEME-AA & $5_{5,0}-5_{4,1}$ & 215668.751 & 34.6 & 0.92 & $\dagger$ & & \\
\hline tEME-AA & $5_{5,0}-5_{4,1}$ & 215669.103 & 34.6 & 0.92 & $\dagger$ & & \\
\hline tEME-AE & $5_{5,1}-5_{4,2}$ & 215669.103 & 34.6 & 0.92 & $\dagger$ & & \\
\hline tEME-EE' & $6_{4,3}-5_{3,3}$ & 216054.535 & 26.5 & 3.73 & 216056.4 & 0.95 & \\
\hline tEME-EE & $6_{4,3}-5_{3,3}$ & 216054.868 & 26.5 & 3.73 & $\dagger$ & & \\
\hline tEME-AE & $64,3-5_{3,3}$ & 216055.095 & 26.5 & 3.73 & $\dagger$ & & \\
\hline tEME-EE & $64,2-5_{3,2}$ & 216055.759 & 26.5 & 3.73 & $\dagger$ & & \\
\hline tEME-EE' & $6_{4,2}-5_{3,2}$ & 216056.093 & 26.5 & 3.73 & $\dagger$ & & \\
\hline tEME-AE & $6_{4,2}-5_{3,2}$ & 216056.319 & 26.5 & 3.73 & $\dagger$ & & \\
\hline tEME-EA & $6_{4,3}-5_{3,3}$ & 216058.222 & 26.5 & 3.69 & $216059.3^{\dagger}$ & 1.14 & \\
\hline tEME-EA & $6_{4,2}-5_{3,2}$ & 216058.519 & 26.5 & 3.69 & $\dagger$ & & \\
\hline tEME-AA & $6_{4,3}-5_{3,2}$ & 216058.597 & 26.5 & 3.73 & $\dagger$ & & \\
\hline tEME-AA & $64,2-5,3,3$ & 216058.930 & 26.5 & 3.73 & $\dagger$ & & \\
\hline tEME-EE & $12_{3,9}-11_{2,10}$ & 217007.530 & 40.5 & 4.14 & 217007.6 & 0.49 & \\
\hline tEME-EE' & $12_{3,9}-11_{2,10}$ & 217007.763 & 40.5 & 4.12 & $\dagger$ & & \\
\hline tEME-AE & $12_{3,9}-11_{2,10}$ & 217007.939 & 40.5 & 4.13 & $\dagger$ & & \\
\hline tEME-EA & $12_{3,9}-11_{2,10}$ & 217009.762 & 40.5 & 4.16 & 217010.6 & 0.54 & \\
\hline tEME-AA & $12_{3,9}-11_{2,10}$ & 217010.054 & 40.5 & 4.16 & ${ }^{\dagger}$ & & \\
\hline tEME-AA & $30_{1,29}-29_{2,28}$ & 217164.465 & 182.5 & 13.55 & 217165.1 & 2.68 & $\mathrm{CH}_{3} \mathrm{OCOH}$ \\
\hline tEME-EA & $30_{1,29}-29_{2,28}$ & 217164.508 & 182.5 & 13.55 & $\dagger$ & & \\
\hline tEME-AE & $30_{1,29}-29_{2,28}$ & 217165.360 & 182.5 & 13.55 & $\dagger$ & & \\
\hline tEME-EE & $30_{1,29}-29_{2,28}$ & 217165.402 & 182.5 & 13.55 & $\dagger$ & & \\
\hline tEME-EE' & $30_{1,29}-29_{2,28}$ & 217165.402 & 182.5 & 13.55 & $\dagger$ & & \\
\hline tEME-AA & $28_{0,28}-27_{1,27}$ & 217940.650 & 154.7 & 22.59 & 217940.7 & 1.46 & \\
\hline tEME-EA & $28_{0,28}-27_{1,27}$ & 217940.651 & 154.7 & 22.59 & $\dagger$ & & \\
\hline tEME-AE & $28_{0,28}-27_{1,27}$ & 217940.758 & 154.7 & 22.59 & $\dagger$ & & \\
\hline
\end{tabular}


B. Tercero et al.: Trans ethyl methyl ether in space

Table A.1. continued.

\begin{tabular}{|c|c|c|c|c|c|c|c|}
\hline Species & $\begin{array}{c}\text { Transition } \\
J_{K_{\mathrm{a}}, K_{\mathrm{c}}}-J_{K_{\mathrm{a}}^{\prime}, K_{\mathrm{c}}^{\prime}}^{\prime}\end{array}$ & $\begin{array}{c}\text { Predicted } \\
\text { frequency }(\mathrm{MHz})\end{array}$ & $\begin{array}{r}E_{\text {upp }} \\
(\mathrm{K})\end{array}$ & $S_{i j}$ & $\begin{array}{c}\text { Observed } \\
\text { frequency }(\mathrm{MHz})\end{array}$ & $\begin{array}{c}T \\
(\mathrm{~K})\end{array}$ & Blends \\
\hline tEME-EE & $28_{0,28}-27_{1,27}$ & 217940.759 & 154.7 & 22.59 & $\bar{\dagger}$ & & \\
\hline tEME-EE' & $28_{0,28}-27_{1,27}$ & 217940.759 & 154.7 & 22.59 & $\dagger$ & & \\
\hline tEME-EE' & $22_{2,21}-21_{1,20}$ & 219893.140 & 102.2 & 8.31 & 219893.0 & 0.86 & \\
\hline tEME-EE & $22_{2,21}-21_{1,20}$ & 219893.140 & 102.2 & 8.31 & $\dagger$ & & \\
\hline tEME-AE & $22_{2,21}-21_{1,20}$ & 219893.263 & 102.2 & 8.31 & $\dagger$ & & \\
\hline tEME-EA & $22_{2,21}-21_{1,20}$ & 219894.511 & 102.2 & 8.31 & $219894.5 \dagger$ & 0.66 & \\
\hline tEME-AA & $22_{2,21}-21_{1,20}$ & 219894.635 & 102.2 & 8.31 & $\dagger$ & & \\
\hline tEME-EE' & $28_{1,28}-27_{0,27}$ & 222861.487 & 154.8 & 22.63 & 222861.2 & 3.06 & $\mathrm{CH}_{3} \mathrm{OCHO}$ \\
\hline tEME-EE & $28_{1,28}-27_{0,27}$ & 222861.487 & 154.8 & 22.63 & $\dagger$ & & \\
\hline tEME-AE & $28_{1,28}-27_{0,27}$ & 222861.500 & 154.8 & 22.63 & $\dagger$ & & \\
\hline tEME-EA & $28_{1,28}-27_{0,27}$ & 222861.655 & 154.8 & 22.63 & $\dagger$ & & \\
\hline tEME-AA & $28_{1,28}-27_{0,27}$ & 222861.668 & 154.8 & 22.63 & $\dagger$ & & \\
\hline tEME-EE' & $13_{3,11}-12_{2,10}$ & 222980.574 & 45.5 & 4.38 & $\ldots$ & $\ldots$ & $\mathrm{CH}_{3} \mathrm{O}^{13} \mathrm{COH}$ \\
\hline tEME-EE & $13_{3,11}-12_{2,10}$ & 222980.720 & 45.5 & 4.39 & $\ldots$ & $\ldots$ & " \\
\hline tEME-AE & $13_{3,11}-12_{2,10}$ & 222980.951 & 45.5 & 4.39 & $\ldots$ & $\ldots$ & $"$ \\
\hline tEME-EA & $13_{3,11}-12_{2,10}$ & 222983.368 & 45.5 & 4.40 & $\ldots$ & $\ldots$ & " \\
\hline tEME-AA & $13_{3,11}-12_{2,10}$ & 222983.672 & 45.5 & 4.40 & $\ldots$ & $\ldots$ & $"$ \\
\hline tEME-EE & $16_{2,14}-15_{1,15}$ & 223403.761 & 57.4 & 2.48 & 223403.5 & 0.82 & $\mathrm{CH}_{3} \mathrm{OCH}_{3}$ \\
\hline tEME-EE' & $16_{2,14}-15_{1,15}$ & 223403.762 & 57.4 & 2.48 & $\dagger$ & & \\
\hline tEME-AE & $16_{2,14}-15_{1,15}$ & 223404.001 & 57.4 & 2.48 & $\dagger$ & & \\
\hline tEME-EA & $16_{2,14}-15_{1,15}$ & 223405.432 & 57.4 & 2.48 & $\ldots$ & $\ldots$ & $\mathrm{CH}_{3} \mathrm{OCH}_{3}$ \\
\hline tEME-AA & $16_{2,14}-15_{1,15}$ & 223405.671 & 57.4 & 2.48 & $\ldots$ & $\ldots$ & \\
\hline tEME-EE' & $74,4-6_{3,4}$ & 224103.753 & 29.2 & 3.89 & 224104.2 & 1.49 & \\
\hline tEME-EE & $7_{4,4}-6_{3,4}$ & 224104.093 & 29.2 & 3.88 & $\dagger$ & & \\
\hline tEME-AE & $74,4-63,4$ & 224104.315 & 29.2 & 3.89 & $\dagger$ & & \\
\hline tEME-EE & $74,3-63,3$ & 224104.926 & 29.2 & 3.88 & $\dagger$ & & \\
\hline tEME-EE' & $7_{4,3}-6_{3,3}$ & 224105.266 & 29.2 & 3.89 & $\dagger$ & & \\
\hline tEME-AE & $7_{4,3}-6_{3,3}$ & 224105.490 & 29.2 & 3.89 & $\dagger$ & & \\
\hline tEME-AA & $7_{4,4}-6_{3,3}$ & 224107.456 & 29.2 & 3.90 & $224108.0^{\dagger}$ & 1.12 & \\
\hline tEME-EA & $74,4-63,4$ & 224107.546 & 29.2 & 3.58 & $\dagger$ & & \\
\hline tEME-EA & $7_{4,3}-6_{3,3}$ & 224107.581 & 29.2 & 3.58 & $\dagger$ & & \\
\hline tEME-AA & $7_{4,3}-6_{3,4}$ & 224108.457 & 29.2 & 3.90 & $\dagger$ & & \\
\hline tEME-EE & $13_{3,10}-12_{2,11}$ & 225283.674 & 45.5 & 4.33 & 225283.2 & 1.41 & $\mathrm{CH}_{3} \mathrm{CH}_{2} \mathrm{OH}$ \\
\hline tEME-EE' & $13_{3,10}-12_{2,11}$ & 225283.820 & 45.5 & 4.33 & $\dagger$ & & \\
\hline tEME-AE & $13_{3,10}-12_{2,11}$ & 225284.043 & 45.5 & 4.33 & $\dagger$ & & \\
\hline tEME-EA & $13_{3,10}-12_{2,11}$ & 225285.990 & 45.5 & 4.34 & $\dagger$ & & \\
\hline tEME-AA & $13_{3,10}-12_{2,11}$ & 225286.286 & 45.5 & 4.34 & $\dagger$ & & \\
\hline tEME-EE' & $23_{2,22}-22_{1,21}$ & 225494.508 & 111.0 & 8.90 & 225494.6 & 0.72 & \\
\hline tEME-EE & $23_{2,22}-22_{1,21}$ & 225494.508 & 111.0 & 8.90 & $\dagger$ & & \\
\hline tEME-AE & $23_{2,22}-22_{1,21}$ & 225494.625 & 111.0 & 8.90 & $\dagger$ & & \\
\hline tEME-EA & $23_{2,22}-22_{1,21}$ & 225495.848 & 111.0 & 8.90 & $\dagger$ & & \\
\hline tEME-AA & $23_{2,22}-22_{1,21}$ & 225495.965 & 111.0 & 8.90 & $\dagger$ & & \\
\hline tEME-EA & $29_{0,29}-28_{1,28}$ & 226057.836 & 165.7 & 23.62 & 226058.4 & 3.50 & $\mathrm{CH}_{3} \mathrm{CH}_{2} \mathrm{OH}$ \\
\hline tEME-AA & $29_{0,29}-28_{1,28}$ & 226057.836 & 165.7 & 23.62 & $\dagger$ & & \\
\hline tEME-EE & $29_{0,29}-28_{1,28}$ & 226057.928 & 165.7 & 23.62 & $\dagger$ & & \\
\hline tEME-EE' & $29_{0,29}-28_{1,28}$ & 226057.928 & 165.7 & 23.62 & $\dagger$ & & \\
\hline tEME-AE & $29_{0,29}-28_{1,28}$ & 226057.928 & 165.7 & 23.62 & $\dagger$ & & \\
\hline tEME-AA & $31_{1,30}-30_{2,29}$ & 227090.552 & 194.5 & 14.51 & $\ldots$ & $\ldots$ & $\mathrm{CH}_{3} \mathrm{OH}$ \\
\hline tEME-EA & $31_{1,30}-30_{2,29}$ & 227090.589 & 194.5 & 14.51 & $\ldots$ & $\ldots$ & $"$ \\
\hline tEME-AE & $31_{1,30}-30_{2,29}$ & 227091.390 & 194.5 & 14.51 & $\ldots$ & $\ldots$ & " \\
\hline tEME-EE & $31_{1,30}-30_{2,29}$ & 227091.426 & 194.5 & 14.51 & $\ldots$ & $\ldots$ & $"$ \\
\hline tEME-EE' & $31_{1,30}-30_{2,29}$ & 227091.426 & 194.5 & 14.51 & $\ldots$ & $\ldots$ & $"$ \\
\hline tEME-EE' & $29_{1,29}-28_{0,28}$ & 230291.194 & 165.8 & 23.66 & $\ldots$ & $\ldots$ & $\mathrm{CH}_{3} \mathrm{OH}, \mathrm{CH}_{3} \mathrm{OCOH}$ \\
\hline tEME-EE & $29_{1,29}-28_{0,28}$ & 230291.194 & 165.8 & 23.66 & $\ldots$ & $\ldots$ & $"$ \\
\hline tEME-AE & $29_{1,29}-28_{0,28}$ & 230291.205 & 165.8 & 23.66 & $\ldots$ & $\ldots$ & $"$ \\
\hline tEME-EA & $29_{1,29}-28_{0,28}$ & 230291.345 & 165.8 & 23.66 & $\ldots$ & $\ldots$ & $"$ \\
\hline tEME-AA & $29_{1,29}-28_{0,28}$ & 230291.357 & 165.8 & 23.66 & $\ldots$ & $\ldots$ & $"$ \\
\hline tEME-EE' & $14_{3,12}-13_{2,11}$ & 230486.881 & 50.9 & 4.59 & 230487.1 & 0.58 & $\mathrm{CO}$ \\
\hline tEME-EE & $14_{3,12}-13_{2,11}$ & 230486.975 & 50.9 & 4.59 & $\dagger$ & & \\
\hline tEME-AE & $14_{3,12}-13_{2,11}$ & 230487.227 & 50.9 & 4.59 & $\dagger$ & & \\
\hline tEME-EA & $14_{3,12}-13_{2,11}$ & 230489.569 & 50.9 & 4.59 & $\ldots$ & $\ldots$ & $\mathrm{CO}$ \\
\hline tEME-AA & $14_{3,12}-13_{2,11}$ & 230489.869 & 50.9 & 4.59 & $\ldots$ & $\ldots$ & $" \prime$ \\
\hline tEME-EE' & $24_{2,23}-23_{1,22}$ & 231063.540 & 120.2 & 9.52 & $\ldots$ & $\ldots$ & OCS \\
\hline tEME-EE & $24_{2,23}-23_{1,22}$ & 231063.540 & 120.2 & 9.52 & $\ldots$ & $\ldots$ & " \\
\hline tEME-AE & $24_{2,23}-23_{1,22}$ & 231063.652 & 120.2 & 9.52 & $\ldots$ & $\ldots$ & $"$ \\
\hline tEME-EA & $24_{2,23}-23_{1,22}$ & 231064.846 & 120.2 & 9.52 & $\ldots$ & $\ldots$ & $"$ \\
\hline tEME-EA & $24_{2,23}-23_{1,22}$ & 231064.958 & 120.2 & 9.52 & $\ldots$ & $\ldots$ & $"$ \\
\hline tEME-EE' & $84,5-73,5$ & 232151.207 & 32.3 & 4.03 & 232151.8 & 1.16 & \\
\hline tEME-EE & $8_{4,5}-7_{3,5}$ & 232151.590 & 32.3 & 3.98 & $\dagger$ & & \\
\hline tEME-AE & $84,5-73,5$ & 232151.787 & 32.3 & 4.01 & $\dagger$ & & \\
\hline tEME-EE & $8_{4,4}-7_{3,4}$ & 232152.098 & 32.3 & 3.98 & $\dagger$ & & \\
\hline tEME-EE' & $84,4-73,4$ & 232152.480 & 32.3 & 4.03 & $\dagger$ & & \\
\hline tEME-EA & $84,5-73,4$ & 232152.521 & 32.3 & 1.00 & $\dagger$ & & \\
\hline
\end{tabular}


A\&A 582, L1 (2015)

Table A.1. continued.

\begin{tabular}{|c|c|c|c|c|c|c|c|}
\hline Species & $\begin{array}{c}\text { Transition } \\
J_{K_{\mathrm{a}}, K_{\mathrm{c}}}-J_{K_{\mathrm{a}}^{\prime}, K_{\mathrm{c}}^{\prime}}^{\prime}\end{array}$ & $\begin{array}{c}\text { Predicted } \\
\text { frequency }(\mathrm{MHz})\end{array}$ & $\begin{array}{r}E_{\text {upp }} \\
(\mathrm{K})\end{array}$ & $S_{i j}$ & $\begin{array}{c}\text { Observed } \\
\text { frequency }(\mathrm{MHz})\end{array}$ & $\begin{array}{c}T \\
(\mathrm{~K})\end{array}$ & Blends \\
\hline tEME-AE & $88_{4,4}-7_{3,4}$ & 232152.685 & 32.3 & 4.01 & $\bar{\dagger}$ & & \\
\hline tEME-AA & $8,5-73,4$ & 232154.033 & 32.3 & 4.08 & $\dagger$ & & \\
\hline tEME-EA & $84,4-73,4$ & 232154.362 & 32.3 & 3.08 & $\dagger$ & & \\
\hline tEME-EA & $84,5-73,5$ & 232155.426 & 32.3 & 3.08 & $\dagger$ & & \\
\hline tEME-AA & $8_{4,4}-7_{3,5}$ & 232156.540 & 32.3 & 4.08 & $\ldots$ & $\ldots$ & $\mathrm{CH}_{3} \mathrm{OCOH} v_{t}=1$ \\
\hline tEME-EA & $\begin{array}{l}4,4,4,7,5 \\
84,5\end{array}$ & 232157.268 & 32.3 & 1.00 & $\begin{array}{l}\cdots \\
\ldots\end{array}$ & $\begin{array}{l}\cdots \\
\ldots\end{array}$ & " \\
\hline tEME-EE & $14_{3,11}-13_{2,12}$ & 233622.462 & 51.0 & 4.51 & 233622.5 & 2.10 & $\mathrm{CH}_{3} \mathrm{CH}_{2} \mathrm{CN} v_{13} / v_{21}$ \\
\hline tEME-EE' & $14_{3,11}-13_{2,12}$ & 233622.556 & 51.0 & 4.51 & $\dagger$ & & \\
\hline tEME-AE & $14_{3,11}-13_{2,12}$ & 233622.806 & 51.0 & 4.51 & $\dagger$ & & \\
\hline tEME-EA & $14_{3,11}-13_{2,12}$ & 233624.824 & 51.0 & 4.51 & $\ldots$ & $\ldots$ & $\mathrm{CH}_{3} \mathrm{OCOH} v_{t}=0,1$ \\
\hline tEME-AA & $14_{3,11}-13_{2,12}$ & 233625.121 & 51.0 & 4.51 & $\ldots$ & $\ldots$ & $"$ \\
\hline tEME-EA & $30_{0,30}-29_{1,29}$ & 234130.523 & 177.0 & 24.66 & $\ldots$ & $\ldots$ & $\mathrm{CH}_{3} \mathrm{OCOH}$ \\
\hline tEME-AA & $30_{0,30}-29_{1,29}$ & 234130.524 & 177.0 & 24.66 & $\ldots$ & $\ldots$ & " \\
\hline tEME-EE & $30_{0,30}-29_{1,29}$ & 234130.601 & 177.0 & 24.66 & $\ldots$ & $\ldots$ & " \\
\hline tEME-EE' & $30_{0,30}-29_{1,29}$ & 234130.601 & 177.0 & 24.66 & $\ldots$ & $\ldots$ & " \\
\hline tEME-AE & $30_{0,30}-29_{1,29}$ & 234130.602 & 177.0 & 24.66 & $\ldots$ & $\ldots$ & $"$ \\
\hline tEME-EE & $17_{2,15}-16_{1,16}$ & 235247.484 & 64.0 & 2.37 & 235247.5 & 0.49 & U-line \\
\hline tEME-EE' & $17_{2,15}-16_{1,16}$ & 235247.485 & 64.0 & 2.37 & $\dagger$ & & \\
\hline tEME-AE & $17_{2,15}-16_{1,16}$ & 235247.731 & 64.0 & 2.37 & $\dagger$ & & \\
\hline tEME-EA & $17_{2,15}-16_{1,16}$ & 235249.156 & 64.0 & 2.37 & 235249.6 & 0.41 & U-line \\
\hline tEME-AA & $17_{2,15}-16_{1,16}$ & 235249.403 & 64.0 & 2.37 & $\dagger$ & & \\
\hline tEME-EE' & $25_{2,24}-24_{1,23}$ & 236614.358 & 129.8 & 10.19 & 236614.8 & 1.89 & $\mathrm{CH}_{3} \mathrm{O}^{13} \mathrm{COH} v_{t}=1$ \\
\hline tEME-EE & $25_{2,24}-24_{1,23}$ & 236614.358 & 129.8 & 10.19 & $\dagger$ & & \\
\hline tEME-AE & $25_{2,24}-24_{1,23}$ & 236614.464 & 129.8 & 10.19 & $\dagger$ & & \\
\hline tEME-EA & $25_{2,24}-24_{1,23}$ & 236615.628 & 129.8 & 10.19 & $\dagger$ & & \\
\hline tEME-AA & $25_{2,24}-24_{1,23}$ & 236615.733 & 129.8 & 10.19 & $\dagger$ & & \\
\hline tEME-AA & $32_{1,31}-31_{2,30}$ & 236906.877 & 206.9 & 15.50 & 236907.6 & 0.99 & \\
\hline tEME-EA & $32_{1,31}-31_{2,30}$ & 236906.908 & 206.9 & 15.50 & $\dagger$ & & \\
\hline tEME-AE & $32_{1,31}-31_{2,30}$ & 236907.656 & 206.9 & 15.50 & $\dagger$ & & \\
\hline tEME-EE & $32_{1,31}-31_{2,30}$ & 236907.687 & 206.9 & 15.50 & $\dagger$ & & \\
\hline tEME-EE' & $32_{1,31}-31_{2,30}$ & 236907.687 & 206.9 & 15.50 & $\dagger$ & & \\
\hline tEME-EE' & $30_{1,30}-29_{0,29}$ & 237763.517 & 177.1 & 24.69 & 237763.2 & 1.76 & \\
\hline tEME-EE & $30_{1,30}-29_{0,29}$ & 237763.517 & 177.1 & 24.69 & $\dagger$ & & \\
\hline tEME-AE & $30_{1,30}-29_{0,29}$ & 237763.527 & 177.1 & 24.69 & $\dagger$ & & \\
\hline tEME-EA & $30_{1,30}-29_{0,29}$ & 237763.653 & 177.1 & 24.69 & $\dagger$ & & \\
\hline tEME-AA & $30_{1,30}-29_{0,29}$ & 237763.664 & 177.1 & 24.69 & $\dagger$ & & \\
\hline tEME-EE' & $15_{3,13}-14_{2,12}$ & 237865.715 & 56.7 & 4.79 & 237866.1 & 1.40 & \\
\hline tEME-EE & $15_{3,13}-14_{2,12}$ & 237865.778 & 56.7 & 4.80 & $\dagger$ & & \\
\hline tEME-AE & $15_{3,13}-14_{2,12}$ & 237866.042 & 56.7 & 4.80 & $\dagger$ & & \\
\hline tEME-EA & $15_{3,13}-14_{2,12}$ & 237868.339 & 56.7 & 4.80 & $\ldots$ & $\ldots$ & U-line \\
\hline tEME-AA & $15_{3,13}-14_{2,12}$ & 237868.635 & 56.7 & 4.80 & $\ldots$ & $\ldots$ & $"$ \\
\hline tEME-EA & $9_{4,6}-8_{3,5}$ & 240195.817 & 35.8 & 1.64 & 240196.7 & 2.66 & ${ }^{13} \mathrm{CH}_{3} \mathrm{OCOH}$ \\
\hline tEME-EE & $9_{4,5}-8_{3,5}$ & 240196.101 & 35.8 & 3.88 & $\dagger$ & & \\
\hline tEME-EE' & $94,6-83,6$ & 240196.396 & 35.8 & 3.88 & $\dagger$ & & \\
\hline tEME-EE' & $9_{4,5}-8,5$ & 240196.642 & 35.8 & 3.88 & $\dagger$ & & \\
\hline tEME-AE & $9_{4,5}-8_{3,5}$ & 240196.777 & 35.8 & 3.88 & $\dagger$ & & \\
\hline tEME-EE & $9_{4,6}-8_{3,6}$ & 240196.937 & 35.8 & 3.88 & $\dagger$ & & \\
\hline tEME-AE & $9_{4,6}-83,6$ & 240197.045 & 35.8 & 3.88 & $\dagger$ & & \\
\hline tEME-AA & $9_{4,6}-8_{3,5}$ & 240197.196 & 35.8 & 3.88 & $\dagger$ & & \\
\hline tEME-EA & $94,5-8,5$ & 240197.654 & 35.8 & 3.88 & $\dagger$ & & \\
\hline tEME-EA & $9_{4,6}-8_{3,6}$ & 240201.478 & 35.8 & 2.63 & $\ldots$ & $\ldots$ & $\mathrm{CH}_{3} \mathrm{OCOH} v_{t}=1$ \\
\hline tEME-AA & $9_{4,5}-8_{3,6}$ & 240202.719 & 35.8 & 4.28 & $\ldots$ & & $"$ \\
\hline tEME-EA & $9_{4,5}-8_{3,6}$ & 240203.315 & 35.8 & 1.64 & $\ldots$ & $\ldots$ & $"$ \\
\hline tEME-EE & $15_{3,12}-14_{2,13}$ & 242035.318 & 56.8 & 4.68 & $\ldots$ & $\ldots$ & $\mathrm{CH}_{2} \mathrm{DOH} ; \mathrm{CH}_{2} \mathrm{DCN}$ \\
\hline tEME-EE' & $15_{3,12}-14_{2,13}$ & 242035.380 & 56.8 & 4.68 & $\ldots$ & $\ldots$ & " \\
\hline tEME-AE & $15_{3,12}-14_{2,13}$ & 242035.647 & 56.8 & 4.68 & $\ldots$ & $\ldots$ & $"$ \\
\hline tEME-EA & $15_{3,12}-14_{2,13}$ & 242037.704 & 56.8 & 4.68 & $\ldots$ & $\ldots$ & $\mathrm{CH}_{2} \mathrm{DOCOH}$ \\
\hline tEME-AA & $15_{3,12}-14_{2,13}$ & 242038.002 & 56.8 & 4.68 & $\ldots$ & $\ldots$ & " \\
\hline tEME-EE' & $26_{2,25}-25_{1,24}$ & 242161.785 & 139.8 & 10.89 & 242163.3 & 1.86 & \\
\hline tEME-EE & $26_{2,25}-25_{1,24}$ & 242161.785 & 139.8 & 10.89 & $\dagger$ & & \\
\hline tEME-AE & $26_{2,25}-25_{1,24}$ & 242161.884 & 139.8 & 10.89 & $\dagger$ & & \\
\hline tEME-EA & $26_{2,25}-25_{1,24}$ & 242163.015 & 139.8 & 10.89 & $\dagger$ & & \\
\hline tEME-AA & $26_{2,25}-25_{1,24}$ & 242163.114 & 139.8 & 10.89 & $\dagger$ & & \\
\hline tEME-EA & $31_{0,31}-30_{1,30}$ & 242163.369 & 188.7 & 25.69 & $\dagger$ & & \\
\hline tEME-AA & $31_{0,31}-30_{1,30}$ & 242163.371 & 188.7 & 25.69 & $\dagger$ & & \\
\hline tEME-EE & $31_{0,31}-30_{1,30}$ & 242163.434 & 188.7 & 25.69 & $\dagger$ & & \\
\hline tEME-EE' & $31_{0,31}-30_{1,30}$ & 242163.434 & 188.7 & 25.69 & $\dagger$ & & \\
\hline tEME-AE & $31_{0,31}-30_{1,30}$ & 242163.436 & 188.7 & 25.69 & $\dagger$ & & \\
\hline tEME-EE' & $16_{3,14}-15_{2,13}$ & 245103.550 & 62.9 & 4.99 & 245103.4 & 0.92 & \\
\hline tEME-EE & $16_{3,14}-15_{2,13}$ & 245103.592 & 62.9 & 4.99 & $\dagger$ & & \\
\hline tEME-AE & $16_{3,14}-15_{2,13}$ & 245103.864 & 62.9 & 4.99 & $\dagger$ & & \\
\hline tEME-EA & $16_{3,14}-15_{2,13}$ & 245106.133 & 62.9 & 4.99 & $245106.4^{\dagger}$ & 1.13 & $\mathrm{CH}_{3} \mathrm{OCOH} v_{t}=2$ \\
\hline tEME-EA & $16_{3,14}-15_{2,13}$ & 245106.426 & 62.9 & 5.00 & $\dagger$ & & \\
\hline
\end{tabular}


B. Tercero et al.: Trans ethyl methyl ether in space

Table A.1. continued.

\begin{tabular}{|c|c|c|c|c|c|c|c|}
\hline Species & $\begin{array}{c}\text { Transition } \\
J_{K_{\mathrm{a}}, K_{\mathrm{c}}}-J_{K_{\mathrm{a}}^{\prime}, K_{\mathrm{c}}^{\prime}}^{\prime}\end{array}$ & $\begin{array}{c}\text { Predicted } \\
\text { frequency }(\mathrm{MHz})\end{array}$ & $\begin{array}{r}E_{\text {upp }} \\
(\mathrm{K})\end{array}$ & $S_{i j}$ & $\begin{array}{c}\text { Observed } \\
\text { frequency }(\mathrm{MHz})\end{array}$ & $\begin{array}{c}T \\
(\mathrm{~K})\end{array}$ & Blends \\
\hline tEME-EE' & $31_{1,31}-30_{0,30}$ & 245274.088 & 188.8 & 25.71 & 245274.0 & 2.44 & \\
\hline tEME-EE & $31_{1,31}-30_{0,30}$ & 245274.088 & 188.8 & 25.71 & $\dagger$ & & \\
\hline tEME-AE & $31_{1,31}-30_{0,30}$ & 245274.098 & 188.8 & 25.71 & $\dagger$ & & \\
\hline tEME-EA & $31_{1,31}-30_{0,30}$ & 245274.211 & 188.8 & 25.71 & $\dagger$ & & \\
\hline tEME-AA & $31_{1,31}-30_{0,30}$ & 245274.221 & 188.8 & 25.71 & $\dagger$ & & \\
\hline tEME-AA & $33_{1,32}-32_{2,31}$ & 246605.346 & 219.6 & 16.51 & 246605.6 & 0.92 & \\
\hline tEME-EA & $33_{1,32}-32_{2,31}$ & 246605.372 & 219.6 & 16.51 & $\dagger$ & & \\
\hline tEME-AE & $33_{1,32}-32_{2,31}$ & 246606.066 & 219.6 & 16.51 & $\dagger$ & & \\
\hline tEME-EE & $33_{1,32}-32_{2,31}$ & 246606.092 & 219.6 & 16.51 & $\dagger$ & & \\
\hline tEME-EE' & $33_{1,32}-32_{2,31}$ & 246606.092 & 219.6 & 16.51 & $\dagger$ & & \\
\hline
\end{tabular}


A\&A 582, L1 (2015)

Table A.2. Lines of trans- $\mathrm{CH}_{3} \mathrm{CH}_{2} \mathrm{OCH}_{3}$ in $30 \mathrm{~m}$ data.

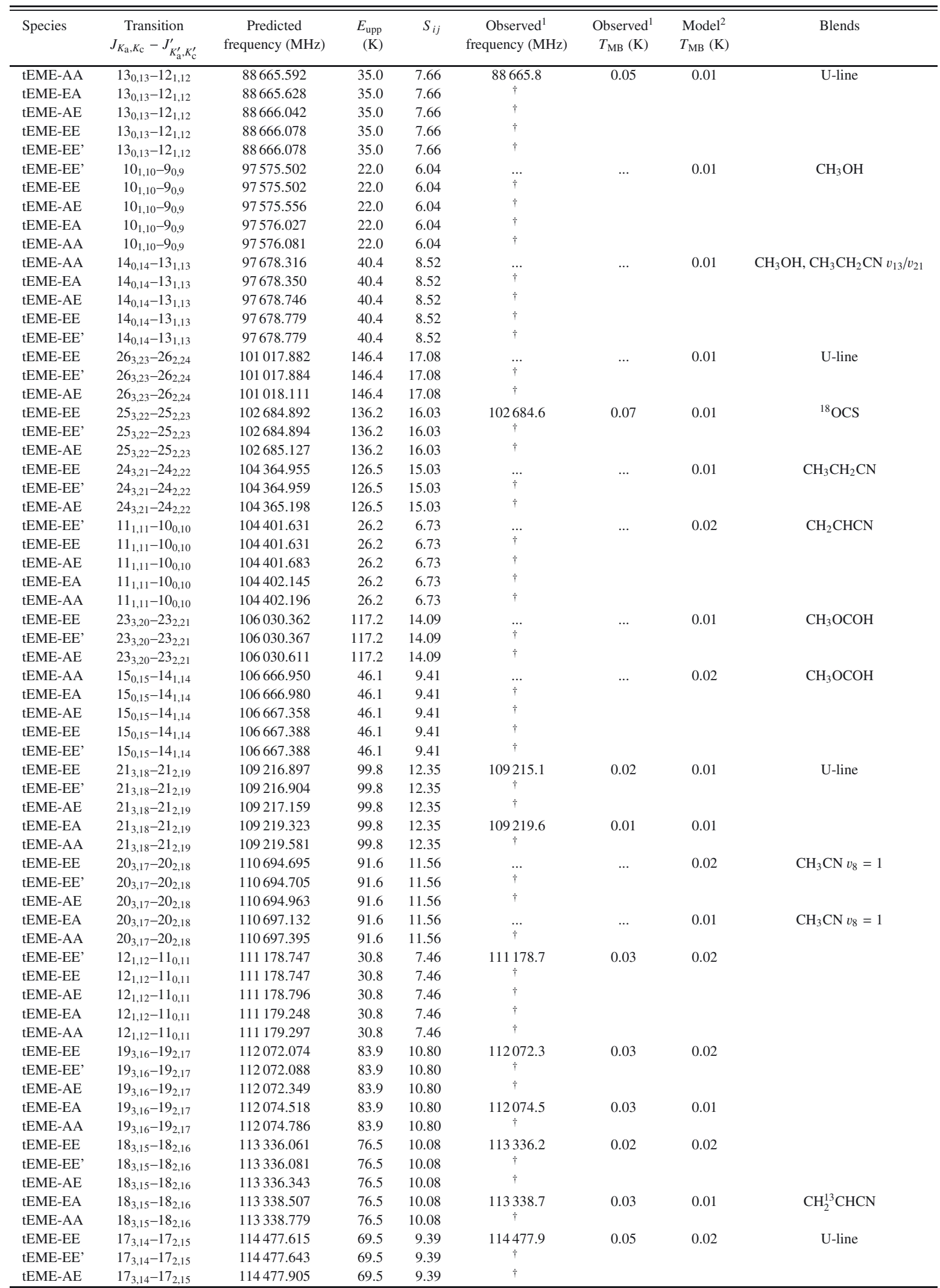

Notes. Lines of trans- $\mathrm{CH}_{3} \mathrm{CH}_{2} \mathrm{OCH}_{3}$ (tEME) ground state present in the spectral scan of Orion $\mathrm{KL}$ from the $30 \mathrm{~m}$ telescope. Column 1 indicates the species, Col. 2 gives the transition, Col. 3 the predicted frequency, Col. 4 upper level energy, Col. 5 the line strength, Col. 6 observed frequency at the peak channel of the line (relative to a $v_{\mathrm{LSR}}$ of $+7.5 \mathrm{~km} \mathrm{~s}^{-1}$ ), Col. 7 main beam temperature at the peak channel of the line, and Col. 8 shows blends with other molecular species. ${ }^{1)}$ Observed frequencies and intensities are not provided for features that appear totally blended with lines from other species. ${ }^{(2)}$ We address all features provided by our model with $T_{\mathrm{MB}}>0.01 \mathrm{~K}, T_{\mathrm{MB}}>0.02 \mathrm{~K}$, and $T_{\mathrm{MB}}>0.03 \mathrm{~K}$ in the frequency ranges between $80.7-116,122.7-150$, and $150-306.7 \mathrm{GHz}$, respectively. ${ }^{(\dagger)}$ Blended with previous line. 
B. Tercero et al.: Trans ethyl methyl ether in space

Table A.2. continued.

\begin{tabular}{|c|c|c|c|c|c|c|c|c|}
\hline Species & $\begin{array}{c}\text { Transition } \\
J_{K_{\mathrm{a}}, K_{\mathrm{c}}}-J_{K_{\mathrm{a}}^{\prime}, K_{\mathrm{c}}^{\prime}}^{\prime}\end{array}$ & $\begin{array}{c}\text { Predicted } \\
\text { frequency }(\mathrm{MHz})\end{array}$ & $\begin{array}{r}E_{\text {upp }} \\
(\mathrm{K})\end{array}$ & $S_{i j}$ & $\begin{array}{c}\text { Observed }^{1} \\
\text { frequency }(\mathrm{MHz})\end{array}$ & $\begin{array}{c}\text { Observed }^{1} \\
T_{\mathrm{MB}}(\mathrm{K})\end{array}$ & $\begin{array}{c}\text { Model }^{2} \\
T_{\mathrm{MB}}(\mathrm{K})\end{array}$ & Blends \\
\hline tEME-EA & $17_{3,14}-17_{2,15}$ & 114480.057 & 69.5 & 9.39 & $\ldots$ & $\ldots$ & 0.01 & $\mathrm{CH}_{3} \mathrm{COOCH}_{3}$ \\
\hline tEME-AA & $17_{3,14}-17_{2,15}$ & 114480.333 & 69.5 & 9.39 & $\dagger$ & & & \\
\hline tEME-AE & $20_{1,19}-19_{2,18}$ & 114717.852 & 83.4 & 6.11 & 114718.0 & 0.06 & 0.01 & $\mathrm{CH}_{3} \mathrm{CHO} v_{t}=1$ \\
\hline tEME-EE & $20_{1,19}-19_{2,18}$ & 114717.961 & 83.4 & 6.11 & $\dagger$ & & & \\
\hline tEME-EE' & $20_{1,19}-19_{2,18}$ & 114717.962 & 83.4 & 6.11 & $\dagger$ & & & \\
\hline tEME-EE & $16_{3,13}-16_{2,14}$ & 115491.673 & 62.9 & 8.73 & $\ldots$ & $\ldots$ & 0.02 & $\mathrm{CH}_{3} \mathrm{CHO}$ \\
\hline tEME-EE' & $16_{3,13}-16_{2,14}$ & 115491.713 & 62.9 & 8.73 & $\dagger$ & & & \\
\hline tEME-AE & $16_{3,13}-16_{2,14}$ & 115491.972 & 62.9 & 8.73 & $\dagger$ & & & \\
\hline tEME-EA & $16_{3,13}-16_{2,14}$ & 115494.106 & 62.9 & 8.73 & $\ldots$ & $\ldots$ & 0.02 & $\mathrm{CH}_{3} \mathrm{CHO}$ \\
\hline tEME-AA & $16_{3,13}-16_{2,14}$ & 115494.385 & 62.9 & 8.73 & $\dagger$ & & & \\
\hline tEME-AA & $16_{0,16}-15_{1,15}$ & 115618.006 & 52.2 & 10.34 & 115618.4 & 0.07 & 0.04 & \\
\hline tEME-EA & $16_{0,16}-15_{1,15}$ & 115618.033 & 52.2 & 10.34 & $\dagger$ & & & \\
\hline tEME-AE & $16_{0,16}-15_{1,15}$ & 115618.391 & 52.2 & 10.34 & $\dagger$ & & & \\
\hline tEME-EE & $16_{0,16}-15_{1,15}$ & 115618.417 & 52.2 & 10.34 & $\dagger$ & & & \\
\hline tEME-EE' & $16_{0,16}-15_{1,15}$ & 115618.417 & 52.2 & 10.34 & $\dagger$ & & & \\
\hline tEME-EE' & $19_{3,17}-19_{2,18}$ & 123318.746 & 83.8 & 10.04 & 123318.8 & 0.02 & 0.02 & \\
\hline tEME-EE & $19_{3,17}-19_{2,18}$ & 123318.760 & 83.8 & 10.04 & $\dot{\dagger}$ & & & \\
\hline tEME-AE & $19_{3,17}-19_{2,18}$ & 123319.043 & 83.8 & 10.04 & ${ }^{\dagger}$ & & & \\
\hline tEME-EA & $19_{3,17}-19_{2,18}$ & 123321.168 & 83.8 & 10.04 & 123321.3 & 0.04 & 0.01 & $\mathrm{CH}_{3} \mathrm{O}^{13} \mathrm{COH}$ \\
\hline tEME-AA & $19_{3,17}-19_{2,18}$ & 123321.458 & 83.8 & 10.04 & $\dagger$ & & & \\
\hline tEME-EE' & $20_{3,18}-20_{2,19}$ & 124043.337 & 91.6 & 10.58 & 124043.5 & 0.03 & 0.02 & $\mathrm{CH}_{3} \mathrm{COOH} v_{t}=1$ \\
\hline tEME-EE & $20_{3,18}-20_{2,19}$ & 124043.347 & 91.6 & 10.58 & + & & & \\
\hline tEME-AE & $20_{3,18}-20_{2,19}$ & 124043.631 & 91.6 & 10.58 & $\dagger$ & & & \\
\hline tEME-EA & $20_{3,18}-20_{2,19}$ & 124045.738 & 91.6 & 10.58 & 124046.0 & 0.02 & 0.01 & \\
\hline tEME-AA & $20_{3,18}-20_{2,19}$ & 124046.027 & 91.6 & 10.58 & $\dagger$ & & & \\
\hline tEME-AA & $17_{0,17}-16_{1,16}$ & 124519.803 & 58.7 & 11.29 & 124519.5 & 0.19 & 0.05 & U-line \\
\hline tEME-EA & $17_{0,17}-16_{1,16}$ & 124519.826 & 58.7 & 11.29 & $\dagger$ & & & \\
\hline tEME-AE & $17_{0,17}-16_{1,16}$ & 124520.163 & 58.7 & 11.29 & $\dagger$ & & & \\
\hline tEME-EE & $17_{0,17}-16_{1,16}$ & 124520.186 & 58.7 & 11.29 & $\dagger$ & & & \\
\hline tEME-EE' & $17_{0,17}-16_{1,16}$ & 124520.186 & 58.7 & 11.29 & $\dagger$ & & & \\
\hline tEME-EE' & $14_{1,14}-13_{0,13}$ & 124648.594 & 41.0 & 9.02 & 124649.0 & 0.06 & 0.04 & \\
\hline tEME-EE & $14_{1,14}-13_{0,13}$ & 124648.594 & 41.0 & 9.02 & $\dagger$ & & & \\
\hline tEME-AE & $14_{1,14}-13_{0,13}$ & 124648.637 & 41.0 & 9.02 & $\dagger$ & & & \\
\hline tEME-EA & $14_{1,14}-13_{0,13}$ & 124649.062 & 41.0 & 9.02 & $\dagger$ & & & \\
\hline tEME-AA & $14_{1,14}-13_{0,13}$ & 124649.106 & 41.0 & 9.02 & $\dagger$ & & & \\
\hline tEME-EE' & $21_{3,19}-21_{2,20}$ & 124866.195 & 99.7 & 11.10 & $\ldots$ & $\ldots$ & 0.02 & $\mathrm{SO}_{2}$ \\
\hline tEME-EE & $21_{3,19}-21_{2,20}$ & 124866.202 & 99.7 & 11.10 & $\dagger$ & & & \\
\hline tEME-AE & $21_{3,19}-21_{2,20}$ & 124866.487 & 99.7 & 11.10 & $\dagger$ & & & \\
\hline tEME-EA & $21_{3,19}-21_{2,20}$ & 124868.576 & 99.7 & 11.10 & $\ldots$ & $\ldots$ & 0.01 & $\mathrm{SO}_{2}$ \\
\hline tEME-AA & $21_{3,19}-21_{2,20}$ & 124868.864 & 99.7 & 11.10 & $\dagger$ & & & \\
\hline tEME-AA & $21_{1,20}-20_{2,19}$ & 125001.226 & 91.6 & 6.68 & $\ldots$ & $\ldots$ & 0.01 & $\mathrm{CH}_{3} \mathrm{OCOH}$ \\
\hline tEME-EA & $21_{1,20}-20_{2,19}$ & 125001.329 & 91.6 & 6.68 & $\doteqdot$ & & & \\
\hline tEME-AE & $21_{1,20}-20_{2,19}$ & 125002.535 & 91.6 & 6.68 & $\ldots$ & $\ldots$ & 0.02 & $\mathrm{CH}_{3} \mathrm{OCOH}$ \\
\hline tEME-EE & $21_{1,20}-20_{2,19}$ & 125002.638 & 91.6 & 6.68 & $\dagger$ & & & \\
\hline tEME-EE' & $21_{1,20}-20_{2,19}$ & 125002.638 & 91.6 & 6.68 & $\dagger$ & & & \\
\hline tEME-EE' & $7_{2,6}-6_{1,5}$ & 125433.445 & 15.4 & 2.61 & $\ldots$ & $\ldots$ & 0.01 & $\mathrm{CH}_{3} \mathrm{CH}_{2} \mathrm{CN}, \mathrm{SO}_{2}$ \\
\hline tEME-EE & $7_{2,6}-6_{1,5}$ & 125433.472 & 15.4 & 2.61 & $\dagger$ & & & \\
\hline tEME-AE & $7_{2,6}-6_{1,5}$ & 125433.646 & 15.4 & 2.61 & $\dagger$ & & & \\
\hline tEME-EE' & $22_{3,20}-22_{2,21}$ & 125793.386 & 108.2 & 11.61 & $\ldots$ & $\ldots$ & 0.02 & $\mathrm{CH}_{3} \mathrm{CH}_{2} \mathrm{CN} v_{13} / v_{21}$ \\
\hline tEME-EE & $22_{3,20}-22_{2,21}$ & 125793.392 & 108.2 & 11.61 & $\dagger$ & & & \\
\hline tEME-AE & $22_{3,20}-22_{2,21}$ & 125793.677 & 108.2 & 11.61 & $\dagger$ & & & \\
\hline tEME-EA & $22_{3,20}-22_{2,21}$ & 125795.748 & 108.2 & 11.61 & $\ldots$ & $\ldots$ & 0.01 & $\mathrm{CH}_{3} \mathrm{CH}_{2} \mathrm{CN} v_{13} / v_{21}$ \\
\hline tEME-AA & $22_{3,20}-22_{2,21}$ & 125796.036 & 108.2 & 11.61 & $\dagger$ & & & \\
\hline tEME-EE' & $23_{3,21}-23_{2,22}$ & 126830.671 & 117.1 & 12.11 & $\ldots$ & $\ldots$ & 0.02 & $\mathrm{HC}^{13} \mathrm{CCN}$ \\
\hline tEME-EE & $23_{3,21}-23_{2,22}$ & 126830.675 & 117.1 & 12.11 & $\dagger$ & & & \\
\hline tEME-AE & $23_{3,21}-23_{2,22}$ & 126830.960 & 117.1 & 12.11 & $\dagger$ & & & \\
\hline tEME-EA & $23_{3,21}-23_{2,22}$ & 126833.013 & 117.1 & 12.11 & $\ldots$ & $\ldots$ & 0.01 & $\mathrm{HC}^{13} \mathrm{CCN}$ \\
\hline tEME-AA & $23_{3,21}-23_{2,22}$ & 126833.301 & 117.1 & 12.11 & $\dagger$ & & & \\
\hline tEME-EE' & $24_{3,22}-24_{2,23}$ & 127983.452 & 126.4 & 12.59 & 127983.6 & 0.07 & 0.02 & U-line \\
\hline tEME-EE & $24_{3,22}-24_{2,23}$ & 127983.456 & 126.4 & 12.59 & $\dagger$ & & & \\
\hline tEME-AE & $24_{3,22}-24_{2,23}$ & 127983.741 & 126.4 & 12.59 & $\dagger$ & & & \\
\hline tEME-EA & $24_{3,22}-24_{2,23}$ & 127985.776 & 126.4 & 12.59 & 127986.1 & 0.06 & 0.01 & $\mathrm{NH}_{2} \mathrm{CHO} v_{12}=1$ \\
\hline tEME-AA & $24_{3,22}-24_{2,23}$ & 127986.063 & 126.4 & 12.59 & $\dagger$ & & & \\
\hline tEME-EE' & $25_{3,23}-25_{2,24}$ & 129256.739 & 136.1 & 13.06 & 129256.8 & 0.04 & 0.02 & \\
\hline tEME-EE & $25_{3,23}-25_{2,24}$ & 129256.741 & 136.1 & 13.06 & $\dagger$ & & & \\
\hline tEME-AE & $25_{3,23}-25_{2,24}$ & 129257.027 & 136.1 & 13.06 & $\dagger$ & & & \\
\hline tEME-EA & $25_{3,23}-25_{2,24}$ & 129259.044 & 136.1 & 13.06 & 129259.3 & 0.04 & 0.01 & \\
\hline tEME-AA & $25_{3,23}-25_{2,24}$ & 129259.331 & 136.0 & 13.06 & $\dot{\dagger}$ & & & \\
\hline tEME-EE' & $26_{3,24}-26_{2,25}$ & 130655.101 & 146.1 & 13.52 & $\ldots$ & $\ldots$ & 0.02 & U-line \\
\hline tEME-EE & $26_{3,24}-26_{2,25}$ & 130655.103 & 146.1 & 13.52 & $\dagger$ & & & \\
\hline tEME-AE & $26_{3,24}-26_{2,25}$ & 130655.389 & 146.1 & 13.52 & $\dagger$ & & & \\
\hline tEME-EA & $26_{3,24}-26_{2,25}$ & 130657.388 & 146.1 & 13.52 & $\ldots$ & $\ldots$ & 0.01 & U-line \\
\hline tEME-AA & $26_{3,24}-26_{2,25}$ & 130657.674 & 146.1 & 13.52 & 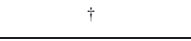 & & & \\
\hline
\end{tabular}


A\&A 582, L1 (2015)

Table A.2. continued.

\begin{tabular}{|c|c|c|c|c|c|c|c|c|}
\hline Species & $\begin{array}{c}\text { Transition } \\
J_{K_{\mathrm{a}}, K_{\mathrm{c}}}-J_{K_{\mathrm{a}}^{\prime}, K_{\mathrm{c}}^{\prime}}^{\prime}\end{array}$ & $\begin{array}{c}\text { Predicted } \\
\text { frequency }(\mathrm{MHz})\end{array}$ & $\begin{array}{r}E_{\text {upp }} \\
(\mathrm{K})\end{array}$ & $S_{i j}$ & $\begin{array}{c}\text { Observed }^{1} \\
\text { frequency }(\mathrm{MHz})\end{array}$ & $\begin{array}{c}\text { Observed }^{1} \\
T_{\mathrm{MB}}(\mathrm{K})\end{array}$ & $\begin{array}{c}\text { Model }^{2} \\
T_{\mathrm{MB}}(\mathrm{K})\end{array}$ & Blends \\
\hline tEME-EE' & $15_{1,15}-14_{0,14}$ & 131372.619 & 46.7 & 9.86 & 131372.7 & 0.05 & 0.05 & \\
\hline tEME-EE & $15_{1,15}-14_{0,14}$ & 131372.619 & 46.7 & 9.86 & $\dagger$ & & & \\
\hline tEME-AE & $15_{1,15}-14_{0,14}$ & 131372.660 & 46.7 & 9.86 & $\dagger$ & & & \\
\hline tEME-EA & $15_{1,15}-14_{0,14}$ & 131373.069 & 46.7 & 9.86 & $\dot{\dagger}$ & & & \\
\hline tEME-AA & $15_{1,15}-14_{0,14}$ & 131373.110 & 46.7 & 9.86 & $\dagger$ & & & \\
\hline tEME-EE' & $27_{3,25}-27_{2,26}$ & 132182.639 & 156.6 & 13.95 & 132182.8 & 0.04 & 0.02 & \\
\hline tEME-EE & $27_{3,25}-27_{2,26}$ & 132182.640 & 156.6 & 13.95 & $\dagger$ & & & \\
\hline tEME-AE & $27_{3,25}-27_{2,26}$ & 132182.927 & 156.6 & 13.95 & $\grave{\dagger}$ & & & \\
\hline tEME-EA & $27_{3,25}-27_{2,26}$ & 132184.906 & 156.6 & 13.95 & 132185.5 & 0.01 & 0.01 & \\
\hline tEME-AA & $27_{3,25}-27_{2,26}$ & 132185.194 & 156.6 & 13.95 & $\dagger$ & & & \\
\hline tEME-EE' & $8_{2,7}-7_{1,6}$ & 132547.336 & 18.5 & 2.87 & 132547.2 & 0.02 & 0.02 & \\
\hline tEME-EE & $8_{2,7}-7_{1,6}$ & 132547.352 & 18.5 & 2.87 & $\dagger$ & & & \\
\hline tEME-AE & $8_{2,7}-7_{1,6}$ & 132547.529 & 18.5 & 2.87 & $\dagger$ & & & \\
\hline tEME-EA & $82,7-7_{1,6}$ & 132548.982 & 18.5 & 2.87 & 132549.0 & 0.02 & 0.01 & \\
\hline tEME-AA & $8_{2,7}-7_{1,6}$ & 132549.167 & 18.5 & 2.87 & 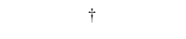 & & & \\
\hline tEME-AA & $18_{0,18}-17_{1,17}$ & 133362.763 & 65.6 & 12.27 & $\ldots$ & $\ldots$ & 0.06 & $\mathrm{O}^{13} \mathrm{CS}, \mathrm{CH}_{2} \mathrm{CHCN} v_{11}=1$ \\
\hline tEME-EA & $18_{0,18}-17_{1,17}$ & 133362.784 & 65.6 & 12.27 & $\dddot{\dagger}$ & & & \\
\hline tEME-AE & $18_{0,18}-17_{1,17}$ & 133363.098 & 65.6 & 12.27 & $\dagger$ & & & \\
\hline tEME-EE & $18_{0,18}-17_{1,17}$ & 133363.118 & 65.6 & 12.27 & $\dagger$ & & & \\
\hline tEME-EE' & $18_{0,18}-17_{1,17}$ & 133363.118 & 65.6 & 12.27 & $\dagger$ & & & \\
\hline tEME-EE' & $28_{3,26}-28_{2,27}$ & 133842.950 & 167.4 & 14.37 & $\ldots$ & $\ldots$ & 0.02 & $\mathrm{CH}_{2} \mathrm{DOH}$ \\
\hline tEME-EE & $28_{3,26}-28_{2,27}$ & 133842.951 & 167.4 & 14.37 & $\dagger$ & & & \\
\hline tEME-AE & $28_{3,26}-28_{2,27}$ & 133843.238 & 167.4 & 14.37 & $\dagger$ & & & \\
\hline tEME-EA & $28_{3,26}-28_{2,27}$ & 133845.198 & 167.4 & 14.37 & $\ldots$ & $\ldots$ & 0.01 & $\mathrm{CH}_{2} \mathrm{DOH}$ \\
\hline tEME-AA & $28_{3,26}-28_{2,27}$ & 133845.486 & 167.4 & 14.37 & $\dagger$ & & & \\
\hline tEME-AA & $22_{1,21}-21_{2,20}$ & 135315.986 & 100.2 & 7.28 & 135316.0 & 0.07 & 0.01 & U-line \\
\hline tEME-EA & $22_{1,21}-21_{2,20}$ & 135316.082 & 100.2 & 7.28 & $\dagger$ & & & \\
\hline tEME-AE & $22_{1,21}-21_{2,20}$ & 135317.259 & 100.2 & 7.28 & 135317.5 & 0.05 & 0.02 & $\mathrm{CH}_{3} \mathrm{CHO} v_{t}=1$ \\
\hline tEME-EE & $22_{1,21}-21_{2,20}$ & 135317.355 & 100.2 & 7.28 & $\dagger$ & & & \\
\hline tEME-EE' & $22_{1,21}-21_{2,20}$ & 135317.355 & 100.2 & 7.28 & $\dagger$ & & & \\
\hline tEME-EE' & $29_{3,27}-29_{2,28}$ & 135639.103 & 178.6 & 14.77 & $\ldots$ & $\ldots$ & 0.02 & $\mathrm{CH}_{3} \mathrm{OCOH} v_{t}=1$ \\
\hline tEME-EE & $29_{3,27}-29_{2,28}$ & 135639.104 & 178.6 & 14.77 & $\dddot{\dagger}$ & & & \\
\hline tEME-AE & $29_{3,27}-29_{2,28}$ & 135639.392 & 178.6 & 14.77 & $\dagger$ & & & \\
\hline tEME-EA & $29_{3,27}-29_{2,28}$ & 135641.333 & 178.6 & 14.77 & $\ldots$ & $\ldots$ & 0.01 & $\mathrm{CH}_{3} \mathrm{OCOH} v_{t}=1$ \\
\hline tEME-AA & $29_{3,27}-29_{2,28}$ & 135641.621 & 178.6 & 14.77 & $\dagger$ & & & \\
\hline tEME-EE' & $30_{3,28}-30_{2,29}$ & 137573.618 & 190.2 & 15.14 & 137573.7 & 0.04 & 0.02 & \\
\hline tEME-EE & $30_{3,28}-30_{2,29}$ & 137573.618 & 190.2 & 15.14 & $\doteqdot$ & & & \\
\hline tEME-AE & $30_{3,28}-30_{2,29}$ & 137573.907 & 190.2 & 15.14 & $\dagger$ & & & \\
\hline tEME-EA & $30_{3,28}-30_{2,29}$ & 137575.829 & 190.2 & 15.14 & 137575.9 & 0.05 & 0.02 & $\mathrm{CH}_{3} \mathrm{COOCH}_{3}$ \\
\hline tEME-AA & $30_{3,28}-30_{2,29}$ & 137576.118 & 190.2 & 15.14 & $\doteqdot$ & & & \\
\hline tEME-EE' & $16_{1,16}-15_{0,15}$ & 138109.231 & 52.7 & 10.73 & 138109.7 & 0.06 & 0.06 & \\
\hline tEME-EE & $16_{1,16}-15_{0,15}$ & 138109.231 & 52.7 & 10.73 & $\dagger$ & & & \\
\hline tEME-AE & $16_{1,16}-15_{0,15}$ & 138109.269 & 52.7 & 10.73 & $\dagger$ & & & \\
\hline tEME-EA & $16_{1,16}-15_{0,15}$ & 138109.661 & 52.7 & 10.73 & $\dagger$ & & & \\
\hline tEME-AA & $16_{1,16}-15_{0,15}$ & 138109.699 & 52.7 & 10.73 & $\dagger$ & & & \\
\hline tEME-EE' & $9_{2,8}-8_{1,7}$ & 139530.181 & 22.0 & 3.15 & $\ldots$ & $\ldots$ & 0.02 & $\mathrm{CH}_{2} \mathrm{DCN}$ \\
\hline tEME-EE & $9_{2,8}-8_{1,7}$ & 139530.191 & 22.0 & 3.15 & $\dot{\dagger}$ & & & \\
\hline tEME-AE & $9_{2,8}-8_{1,7}$ & 139530.369 & 22.0 & 3.15 & $\dagger$ & & & \\
\hline tEME-EA & $9_{2,8}-8_{1,7}$ & 139531.807 & 22.0 & 3.15 & $\ldots$ & $\ldots$ & 0.01 & $\mathrm{CH}_{2} \mathrm{DCN}$ \\
\hline tEME-AA & $9_{2,8}-8_{1,7}$ & 139531.989 & 22.0 & 3.15 & $\grave{\dagger}$ & & & \\
\hline tEME-EE' & $31_{3,29}-31_{2,30}$ & 139648.445 & 202.2 & 15.50 & $\ldots$ & $\ldots$ & 0.02 & $\mathrm{CH}_{3} \mathrm{COCH}_{3}$ \\
\hline tEME-EE & $31_{3,29}-31_{2,30}$ & 139648.445 & 202.2 & 15.50 & $\dagger$ & & & \\
\hline tEME-AE & $31_{3,29}-31_{2,30}$ & 139648.735 & 202.2 & 15.50 & $\dagger$ & & & \\
\hline tEME-EA & $31_{3,29}-31_{2,30}$ & 139650.637 & 202.2 & 15.50 & $\ldots$ & $\ldots$ & 0.01 & $\mathrm{CH}_{3} \mathrm{COCH}_{3}$ \\
\hline tEME-AA & $31_{3,29}-31_{2,30}$ & 139650.927 & 202.2 & 15.50 & 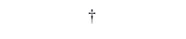 & & & \\
\hline tEME-EE & $8_{2,6}-7_{1,7}$ & 140527.950 & 18.5 & 2.43 & 140528.1 & 0.02 & 0.02 & \\
\hline tEME-EE' & $8_{2,6}-7_{1,7}$ & 140527.966 & 18.5 & 2.43 & $\dagger$ & & & \\
\hline tEME-AE & $8_{2,6}-7_{1,7}$ & 140528.160 & 18.5 & 2.43 & $\dagger$ & & & \\
\hline tEME-EA & $8_{2,6}-7_{1,7}$ & 140529.589 & 18.5 & 2.43 & 140529.6 & 0.03 & 0.01 & U-line \\
\hline tEME-AA & $8,6-7_{1,7}$ & 140529.791 & 18.5 & 2.43 & $\dagger$ & & & \\
\hline tEME-EE' & $32_{3,30}-32_{2,31}$ & 141864.954 & 214.6 & 15.83 & $\ldots$ & $\ldots$ & 0.02 & $\mathrm{CH}_{3} \mathrm{COOH} v_{t}=1$ \\
\hline tEME-EE & $32_{3,30}-32_{2,31}$ & 141864.954 & 214.6 & 15.83 & $\dot{\dagger}$ & & & \\
\hline tEME-AE & $32_{3,30}-32_{2,31}$ & 141865.246 & 214.6 & 15.83 & $\dagger$ & & & \\
\hline tEME-EA & $32_{3,30}-32_{2,31}$ & 141867.127 & 214.6 & 15.83 & $\ldots$ & $\ldots$ & 0.01 & $\mathrm{CH}_{3} \mathrm{COOH} v_{t}=1$ \\
\hline tEME-AA & $32_{3,30}-32_{2,31}$ & 141867.419 & 214.6 & 15.83 & $\dot{\dagger}$ & & & \\
\hline tEME-AA & $19_{0,19}-18_{1,18}$ & 142139.587 & 72.8 & 13.27 & 142139.7 & 0.08 & 0.06 & \\
\hline tEME-EA & $19_{0,19}-18_{1,18}$ & 142139.605 & 72.8 & 13.27 & $\dot{\dagger}$ & & & \\
\hline tEME-AE & $19_{0,19}-18_{1,18}$ & 142139.896 & 72.8 & 13.27 & $\dagger$ & & & \\
\hline tEME-EE & $19_{0,19}-18_{1,18}$ & 142139.914 & 72.8 & 13.27 & $\dagger$ & & & \\
\hline tEME-EE' & $19_{0,19}-18_{1,18}$ & 142139.914 & 72.8 & 13.27 & $\dagger$ & & & \\
\hline tEME-EE' & $3_{3,1}-2_{2,1}$ & 143977.261 & 12.7 & 2.45 & 143977.8 & 0.06 & 0.02 & U-line \\
\hline tEME-EE & $3_{3,1}-22,1$ & 143977.759 & 12.7 & 2.41 & $\dagger$ & & & \\
\hline tEME-AE & $3_{3,1}-2_{2,1}$ & 143977.810 & 12.7 & 2.44 & $\dagger$ & & & \\
\hline
\end{tabular}


B. Tercero et al.: Trans ethyl methyl ether in space

Table A.2. continued.

\begin{tabular}{|c|c|c|c|c|c|c|c|c|}
\hline Species & $\begin{array}{c}\text { Transition } \\
J_{K_{\mathrm{a}}, K_{\mathrm{c}}}-J_{K_{\mathrm{a}}^{\prime}, K_{\mathrm{c}}^{\prime}}^{\prime}\end{array}$ & $\begin{array}{c}\text { Predicted } \\
\text { frequency }(\mathrm{MHz})\end{array}$ & $\begin{array}{r}E_{\text {upp }} \\
(\mathrm{K})\end{array}$ & $S_{i j}$ & $\begin{array}{c}\text { Observed }^{1} \\
\text { frequency }(\mathrm{MHz})\end{array}$ & $\begin{array}{c}\text { Observed }^{1} \\
T_{\mathrm{MB}}(\mathrm{K})\end{array}$ & $\begin{array}{c}\text { Model }^{2} \\
T_{\mathrm{MB}}(\mathrm{K})\end{array}$ & Blends \\
\hline tEME-EA & $33,1-22,0$ & 143979.010 & 12.7 & .72 & 143980.0 & 0.05 & 0.02 & $\mathrm{CH}_{3} \mathrm{COCH}_{3}$ \\
\hline tEME-EE & $3_{3,0}-2_{2,0}$ & 143979.276 & 12.7 & 2.41 & $\dagger$ & & & \\
\hline tEME-EE' & $3_{3,0}-2_{2,0}$ & 143979.774 & 12.7 & 2.45 & $\dagger$ & & & \\
\hline tEME-AE & $3_{3,0}-2_{2,0}$ & 143979.832 & 12.7 & 2.44 & $\dagger$ & & & \\
\hline tEME-AA & $3_{3,1}-2_{2,0}$ & 143980.192 & 12.7 & 2.50 & $\dagger$ & & & \\
\hline tEME-EA & $3_{3,0}-2_{2,0}$ & 143980.533 & 12.7 & 1.78 & $\dagger$ & & & \\
\hline tEME-EE' & $33_{3,31}-33_{2,32}$ & 144223.924 & 227.3 & 16.14 & 144224.0 & 0.02 & 0.01 & \\
\hline tEME-EE & $33_{3,31}-33_{2,32}$ & 144223.925 & 227.3 & 16.14 & $\grave{\dagger}$ & & & \\
\hline tEME-AE & $33_{3,31}-33_{2,32}$ & 144224.218 & 227.3 & 16.14 & $\dagger$ & & & \\
\hline tEME-EE' & $17_{1,17}-16_{0,16}$ & 144871.829 & 59.2 & 11.63 & 144872.1 & 0.06 & 0.06 & \\
\hline tEME-EE & $17_{1,17}-16_{0,16}$ & 144871.829 & 59.2 & 11.63 & $\doteqdot$ & & & \\
\hline tEME-AE & $17_{1,17}-16_{0,16}$ & 144871.864 & 59.2 & 11.63 & $\dagger$ & & & \\
\hline tEME-EA & $17_{1,17}-16_{0,16}$ & 144872.238 & 59.2 & 11.63 & $\dagger$ & & & \\
\hline tEME-AA & $17_{1,17}-16_{0,16}$ & 144872.273 & 59.2 & 11.63 & $\dagger$ & & & \\
\hline tEME-AA & $23_{1,22}-22_{2,21}$ & 145647.137 & 109.2 & 7.92 & 145647.4 & 0.03 & 0.01 & \\
\hline tEME-EA & $23_{1,22}-22_{2,21}$ & 145647.226 & 109.2 & 7.92 & $\dagger$ & & & \\
\hline tEME-AE & $23_{1,22}-22_{2,21}$ & 145648.372 & 109.2 & 7.92 & 145648.6 & 0.03 & 0.02 & \\
\hline tEME-EE & $23_{1,22}-22_{2,21}$ & 145648.461 & 109.2 & 7.92 & $\stackrel{\dagger}{+}$ & & & \\
\hline tEME-EE' & $23_{1,22}-22_{2,21}$ & 145648.462 & 109.2 & 7.92 & $\dagger$ & & & \\
\hline tEME-EE' & $10_{2,9}-9_{1,8}$ & 146383.619 & 25.8 & 3.44 & 146384.0 & 0.03 & 0.02 & \\
\hline tEME-EE & $10_{2,9}-9_{1,8}$ & 146383.626 & 25.8 & 3.44 & 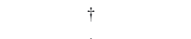 & & & \\
\hline tEME-AE & $10_{2,9}-9_{1,8}$ & 146383.802 & 25.8 & 3.44 & $\dagger$ & & & \\
\hline tEME-EA & $10_{2,9}-9_{1,8}$ & 146385.228 & 25.8 & 3.44 & 146385.5 & 0.03 & 0.02 & \\
\hline tEME-AA & $10_{2,9}-9_{1,8}$ & 146385.408 & 25.8 & 3.44 & $\dagger$ & & & \\
\hline tEME-EE & $36_{4,32}-36_{3,33}$ & 146397.039 & 276.3 & 21.88 & $\ldots$ & $\ldots$ & 0.01 & $\mathrm{SO}_{2}, \mathrm{CH}_{3} \mathrm{OCH}_{3}$ \\
\hline tEME-EE' & $36_{4,32}-36_{3,33}$ & 146397.042 & 276.3 & 21.88 & $\dot{\dagger}$ & & & \\
\hline tEME-AE & $36_{4,32}-36_{3,33}$ & 146397.310 & 276.3 & 21.88 & $\dagger$ & & & \\
\hline tEME-EE' & $34_{3,32}-34_{2,33}$ & 146725.545 & 240.5 & 16.44 & 146725.6 & 0.03 & 0.01 & $\mathrm{SO}^{18} \mathrm{O}$ \\
\hline tEME-EE & $34_{3,32}-34_{2,33}$ & 146725.545 & 240.5 & 16.44 & $\doteqdot$ & & & \\
\hline tEME-AE & $34_{3,32}-34_{2,33}$ & 146725.840 & 240.5 & 16.44 & $\dagger$ & & & \\
\hline tEME-AE & $29_{2,27}-28_{3,26}$ & 146736.865 & 174.4 & 6.43 & $\ldots$ & $\ldots$ & 0.01 & U-line \\
\hline tEME-EE & $29_{2,27}-28_{3,26}$ & 146737.046 & 174.4 & 6.43 & $\dagger$ & & & \\
\hline tEME-EE' & $29_{2,27}-28_{3,26}$ & 146737.048 & 174.4 & 6.43 & $\dagger$ & & & \\
\hline tEME-EE & $35_{4,31}-35_{3,32}$ & 148578.427 & 262.3 & 20.95 & $\ldots$ & $\ldots$ & 0.01 & $\mathrm{CH}_{3} \mathrm{OCOH} v_{t}=1$ \\
\hline tEME-EE' & $35_{4,31}-35_{3,32}$ & 148578.431 & 262.3 & 20.95 & $\dagger$ & & & \\
\hline tEME-AE & $35_{4,31}-35_{3,32}$ & 148578.707 & 262.3 & 20.95 & $\dagger$ & & & \\
\hline tEME-EE' & $35_{3,33}-35_{2,34}$ & 149369.412 & 254.0 & 16.71 & $\ldots$ & $\ldots$ & 0.01 & $\mathrm{CH}_{3} \mathrm{OCOH} v_{t}=1$ \\
\hline tEME-EE & $35_{3,33}-35_{2,34}$ & 149369.412 & 254.0 & 16.71 & $\dagger$ & & & \\
\hline tEME-AE & $35_{3,33}-35_{2,34}$ & 149369.709 & 254.0 & 16.71 & $\dagger$ & & & \\
\hline tEME-EE & $9_{2,7}-8_{1,8}$ & 149921.139 & 22.0 & 2.54 & $\ldots$ & $\ldots$ & 0.02 & $\mathrm{CH}_{3} \mathrm{OCOH}$ \\
\hline tEME-EE' & $9_{2,7}-8_{1,8}$ & 149921.149 & 22.0 & 2.54 & $\dagger$ & & & \\
\hline tEME-AE & $9_{2,7}-8_{1,8}$ & 149921.348 & 22.0 & 2.54 & $\dagger$ & & & \\
\hline tEME-EA & $9_{2,7}-8_{1,8}$ & 149922.787 & 22.0 & 2.54 & $\ldots$ & $\ldots$ & 0.01 & $\mathrm{CH}_{3} \mathrm{OCOH}$ \\
\hline tEME-AA & $9_{2,7}-8_{1,8}$ & 149922.992 & 22.0 & 2.54 & $\dagger$ & & & \\
\hline tEME-EE & $34_{4,30}-34_{3,31}$ & 150661.347 & 248.7 & 20.06 & 150661.4 & 0.04 & 0.02 & U-line \\
\hline tEME-EE' & $34_{4,30}-34_{3,31}$ & 150661.353 & 248.7 & 20.06 & $\dagger$ & & & \\
\hline tEME-AE & $34_{4,30}-34_{3,31}$ & 150661.636 & 248.7 & 20.06 & $\dagger$ & & & \\
\hline tEME-EA & $34_{4,30}-34_{3,31}$ & 150664.261 & 248.7 & 20.06 & 150664.4 & 0.03 & 0.02 & U-line \\
\hline tEME-AA & $34_{4,30}-34_{3,31}$ & 150664.547 & 248.7 & 20.06 & $\dagger$ & & & \\
\hline tEME-AA & $20_{0,20}-19_{1,19}$ & 150845.281 & 80.4 & 14.28 & 150845.4 & 0.08 & 0.09 & \\
\hline tEME-EA & $20_{0,20}-19_{1,19}$ & 150845.296 & 80.4 & 14.28 & $\dagger$ & & & \\
\hline tEME-AE & $20_{0,20}-19_{1,19}$ & 150845.565 & 80.4 & 14.28 & $\dagger$ & & & \\
\hline tEME-EE & $20_{0,20}-19_{1,19}$ & 150845.580 & 80.4 & 14.28 & $\dagger$ & & & \\
\hline tEME-EE' & $20_{0,20}-19_{1,19}$ & 150845.580 & 80.4 & 14.28 & $\dagger$ & & & \\
\hline tEME-EE' & $18_{1,18}-17_{0,17}$ & 151672.109 & 66.0 & 12.56 & 151672.4 & 0.06 & 0.09 & \\
\hline tEME-EE & $18_{1,18}-17_{0,17}$ & 151672.109 & 66.0 & 12.56 & $\dagger$ & & & \\
\hline tEME-AE & $18_{1,18}-17_{0,17}$ & 151672.141 & 66.0 & 12.56 & $\dagger$ & & & \\
\hline tEME-EA & $18_{1,18}-17_{0,17}$ & 151672.495 & 66.0 & 12.56 & $\dagger$ & & & \\
\hline tEME-AA & $18_{1,18}-17_{0,17}$ & 151672.527 & 66.0 & 12.56 & $\dagger$ & & & \\
\hline tEME-EE' & $11_{2,10}-10_{1,9}$ & 153109.700 & 30.1 & 3.73 & 153109.7 & 0.05 & 0.03 & \\
\hline tEME-EE & $11_{2,10}-10_{1,9}$ & 153109.705 & 30.1 & 3.73 & $\dot{\dagger}$ & & & \\
\hline tEME-AE & $11_{2,10}-10_{1,9}$ & 153109.879 & 30.1 & 3.73 & $\doteqdot$ & & & \\
\hline tEME-EA & $11_{2,10}-10_{1,9}$ & 153111.294 & 30.1 & 3.73 & 153111.4 & 0.05 & 0.02 & U-line \\
\hline tEME-AA & $11_{2,10}-10_{1,9}$ & 153111.471 & 30.1 & 3.73 & 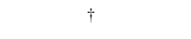 & & & \\
\hline tEME-EE & $32_{4,28}-32_{3,29}$ & 154467.850 & 222.8 & 18.42 & 154468.1 & 0.02 & 0.02 & \\
\hline tEME-EE' & $32_{4,28}-32_{3,29}$ & 154467.860 & 222.8 & 18.42 & $\dagger$ & & & \\
\hline tEME-AE & $32_{4,28}-32_{3,29}$ & 154468.157 & 222.8 & 18.42 & $\dagger$ & & & \\
\hline tEME-AA & $24_{1,23}-23_{2,22}$ & 155980.202 & 118.5 & 8.61 & $\ldots$ & $\ldots$ & 0.03 & $\mathrm{CH}_{3} \mathrm{CH}_{2} \mathrm{CN} v_{12}=1$ \\
\hline tEME-EA & $24_{1,23}-23_{2,22}$ & 155980.284 & 118.5 & 8.61 & $\dagger$ & & & \\
\hline tEME-AE & $24_{1,23}-23_{2,22}$ & 155981.396 & 118.5 & 8.61 & $\ldots$ & $\ldots$ & 0.03 & $\mathrm{CH}_{3} \mathrm{CH}_{2} \mathrm{CN} v_{12}=1$ \\
\hline tEME-EE & $24_{1,23}-23_{2,22}$ & 155981.478 & 118.5 & 8.61 & $\dagger$ & & & \\
\hline tEME-EE' & $24_{1,23}-23_{2,22}$ & 155981.479 & 118.5 & 8.61 & $\dagger$ & & & \\
\hline tEME-EE & $31_{4,27}-31_{3,28}$ & 156169.103 & 210.3 & 17.65 & $\ldots$ & $\ldots$ & 0.02 & $\mathrm{CH}_{3} \mathrm{CH}_{2} \mathrm{CN}$ \\
\hline
\end{tabular}


A\&A 582, L1 (2015)

Table A.2. continued.

\begin{tabular}{|c|c|c|c|c|c|c|c|c|}
\hline Species & $\begin{array}{c}\text { Transition } \\
J_{K_{\mathrm{a}}, K_{\mathrm{c}}}-J_{K_{\mathrm{a}}^{\prime}, K_{\mathrm{c}}^{\prime}}^{\prime}\end{array}$ & $\begin{array}{c}\text { Predicted } \\
\text { frequency }(\mathrm{MHz})\end{array}$ & $\begin{array}{r}E_{\text {upp }} \\
(\mathrm{K})\end{array}$ & $S_{i j}$ & $\begin{array}{c}\text { Observed }^{1} \\
\text { frequency }(\mathrm{MHz})\end{array}$ & $\begin{array}{c}\text { Observed }^{1} \\
T_{\mathrm{MB}}(\mathrm{K})\end{array}$ & $\begin{array}{c}\text { Model }^{2} \\
T_{\mathrm{MB}}(\mathrm{K})\end{array}$ & Blends \\
\hline tEME-EE' & $31_{4,27}-31_{3,28}$ & 156169.114 & 210.3 & 17.65 & $\bar{\dagger}$ & & & \\
\hline tEME-AE & $31_{4,27}-31_{3,28}$ & 156169.418 & 210.3 & 17.65 & $\dagger$ & & & \\
\hline tEME-EE & $30_{4,26}-31_{3,27}$ & 157727.158 & 198.3 & 16.91 & 157727.3 & 0.03 & 0.02 & \\
\hline tEME-EE' & $30_{4,26}-31_{3,27}$ & 157727.174 & 198.3 & 16.91 & $\dagger$ & & & \\
\hline tEME-AE & $30_{4,26}-31_{3,27}$ & 157727.482 & 198.3 & 16.91 & $\dagger$ & & & \\
\hline tEME-EE' & $19_{1,19}-18_{0,18}$ & 158519.756 & 73.2 & 13.52 & $\ldots$ & $\ldots$ & 0.10 & $\mathrm{CH}_{3} \mathrm{OCOH} v_{t}=1, \mathrm{CH}_{3} \mathrm{COCH}_{3}$ \\
\hline tEME-EE & $19_{1,19}-18_{0,18}$ & 158519.756 & 73.2 & 13.52 & $\grave{\dagger}$ & & & \\
\hline tEME-AE & $19_{1,19}-18_{0,18}$ & 158519.786 & 73.2 & 13.52 & $\dagger$ & & & \\
\hline tEME-EA & $19_{1,19}-18_{0,18}$ & 158520.119 & 73.2 & 13.52 & $\dagger$ & & & \\
\hline tEME-AA & $19_{1,19}-18_{0,18}$ & 158520.149 & 73.2 & 13.52 & $\dot{\dagger}$ & & & \\
\hline tEME-EE & $29_{4,25}-29_{3,26}$ & 159140.100 & 186.7 & 16.20 & $\ldots$ & $\ldots$ & 0.03 & U-line \\
\hline tEME-EE' & $29_{4,25}-29_{3,26}$ & 159140.121 & 186.7 & 16.19 & $\dagger$ & & & \\
\hline tEME-AE & $29_{4,25}-29_{3,26}$ & 159140.432 & 186.7 & 16.20 & $\dot{\dagger}$ & & & \\
\hline tEME-EA & $29_{4,25}-29_{3,26}$ & 159143.035 & 186.7 & 16.20 & $\ldots$ & $\ldots$ & 0.02 & U-line \\
\hline tEME-AA & $29_{4,25}-29_{3,26}$ & 159143.357 & 186.7 & 16.20 & $\dagger$ & & & \\
\hline tEME-AA & $21_{0,21}-20_{1,20}$ & 159477.061 & 88.4 & 15.31 & 159477.1 & 0.15 & 0.10 & $\mathrm{CH}_{2} \mathrm{CN}$ \\
\hline tEME-EA & $21_{0,21}-20_{1,20}$ & 159477.074 & 88.4 & 15.31 & $\dagger$ & & & \\
\hline tEME-AE & $21_{0,21}-20_{1,20}$ & 159477.319 & 88.4 & 15.31 & $\dagger$ & & & \\
\hline tEME-EE & $21_{0,21}-20_{1,20}$ & 159477.332 & 88.4 & 15.31 & $\grave{\dagger}$ & & & \\
\hline tEME-EE' & $21_{0,21}-20_{1,20}$ & 159477.332 & 88.4 & 15.31 & $\dagger$ & & & \\
\hline tEME-EE & $10_{2,8}-9_{1,9}$ & 159548.654 & 25.9 & 2.61 & 159548.8 & 0.09 & 0.02 & $\mathrm{CH}_{3} \mathrm{COOH} v_{t}=2$ \\
\hline tEME-EE' & $10_{2,8}-9,9$ & 159548.661 & 25.9 & 2.61 & $\dagger$ & " & " & \\
\hline tEME-AE & $10_{2,8}-9_{1,9}$ & 159548.866 & 25.9 & 2.61 & $\dagger$ & $"$ & $"$ & \\
\hline tEME-EE' & $12_{2,11}-11_{1,10}$ & 159710.946 & 34.7 & 4.05 & $\ldots$ & $\ldots$ & 0.03 & $\mathrm{CH}_{3} \mathrm{CH}_{2} \mathrm{CN} v_{12} / v_{21}$ \\
\hline tEME-EE & $12_{2,11}-11_{1,10}$ & 159710.950 & 34.7 & 4.05 & $\dagger$ & & & \\
\hline tEME-AE & $12_{2,11}-11_{1,10}$ & 159711.121 & 34.7 & 4.05 & $\dagger$ & & & \\
\hline tEME-EA & $12_{2,11}-11_{1,10}$ & 159712.526 & 34.7 & 4.05 & $\ldots$ & $\ldots$ & 0.02 & $\mathrm{CH}_{3} \mathrm{CH}_{2} \mathrm{CN} v_{12} / v_{21}$ \\
\hline tEME-AA & $12_{2,11}-11_{1,10}$ & 159712.699 & 34.7 & 4.05 & $\dagger$ & & & \\
\hline tEME-EE & $28_{4,24}-28_{3,25}$ & 160409.193 & 175.5 & 15.50 & $\ldots$ & $\ldots$ & 0.03 & $\mathrm{CH}_{3} \mathrm{COCH}_{3}$ \\
\hline tEME-EE' & $28_{4,24}-28_{3,25}$ & 160409.221 & 175.5 & 15.50 & $\dagger$ & & & \\
\hline tEME-AE & $28_{4,24}-28_{3,25}$ & 160409.535 & 175.5 & 15.50 & $\dagger$ & & & \\
\hline tEME-EA & $28_{4,24}-28_{3,25}$ & 160412.123 & 175.5 & 15.50 & $\ldots$ & $\ldots$ & 0.02 & $\mathrm{CH}_{3} \mathrm{OCOH} v_{t}=1$ \\
\hline tEME-AA & $28_{4,24}-28_{3,25}$ & 160412.450 & 175.5 & 15.50 & $\dagger$ & & & \\
\hline tEME-EE' & $10_{3,8}-9_{2,7}$ & 199842.884 & 31.6 & 3.55 & 199843.5 & $\ldots$ & 0.05 & $\mathrm{CH}_{3} \mathrm{OCOH} v_{t}=2$ \\
\hline tEME-EE & $10_{3,8}-9_{2,7}$ & 199843.513 & 31.6 & 3.65 & $\dagger$ & & & \\
\hline tEME-AE & $10_{3,8}-9_{2,7}$ & 199843.521 & 31.6 & 3.61 & $\dagger$ & & & \\
\hline tEME-EA & $10_{3,8}-9_{2,7}$ & 199846.703 & 31.6 & 3.78 & $\ldots$ & $\ldots$ & 0.04 & $\mathrm{CH}_{3} \mathrm{OCOH} v_{t}=1$ \\
\hline tEME-AA & $10_{3,8}-9_{2,7}$ & 199847.033 & 31.6 & 3.78 & $\dagger$ & & & \\
\hline tEME-EE' & $4_{4,1}-3_{3,1}$ & 199953.072 & 22.3 & 3.50 & 199953.5 & 0.18 & 0.06 & $\mathrm{CH}_{3} \mathrm{CH}_{2} \mathrm{OH}$ \\
\hline tEME-EE & $4_{4,1}-3_{3,1}$ & 199953.406 & 22.3 & 3.50 & $\dagger$ & & & \\
\hline tEME-AE & $4_{4,1}-3_{3,1}$ & 199953.633 & 22.3 & 3.50 & $\dagger$ & & & \\
\hline tEME-EE & $4_{4,0}-3_{3,0}$ & 199954.307 & 22.3 & 3.50 & 199954.7 & 0.12 & 0.06 & $\mathrm{CH}_{3} \mathrm{CH}_{2} \mathrm{OH}$ \\
\hline tEME-EE' & $4_{4,0}-3_{3,0}$ & 199954.642 & 22.3 & 3.50 & $\dot{\dagger}$ & & & \\
\hline tEME-AE & $4_{4,0}-3_{3,0}$ & 199954.869 & 22.3 & 3.50 & $\dagger$ & & & \\
\hline tEME-EA & $4_{4,1}-3_{3,1}$ & 199956.750 & 22.3 & 3.50 & 199957.2 & 0.19 & 0.08 & $\mathrm{CH}_{2} \mathrm{CHCN} v_{15}=1$ \\
\hline tEME-EA & $4_{4,0}-3_{3,0}$ & 199957.085 & 22.3 & 3.50 & $\dot{\dagger}$ & & & \\
\hline tEME-AA & $4_{4,1}-3_{3,0}$ & 199957.305 & 22.3 & 3.50 & $\dot{\dagger}$ & & & \\
\hline tEME-AA & $4_{4,0}-3_{3,1}$ & 199957.317 & 22.3 & 3.50 & $\dagger$ & & & \\
\hline tEME-EE & $10_{3,7}-9_{2,8}$ & 200603.336 & 31.6 & 3.64 & $\ldots$ & $\ldots$ & 0.05 & U-line \\
\hline tEME-AE & $10_{3,7}-9_{2,8}$ & 200603.932 & 31.6 & 3.59 & $\dagger$ & & & \\
\hline tEME-EE' & $10_{3,7}-9_{2,8}$ & 200603.965 & 31.6 & 3.54 & $\dagger$ & & & \\
\hline tEME-EA & $10_{3,7}-9_{2,8}$ & 200605.130 & 31.6 & 3.76 & $\ldots$ & $\ldots$ & 0.04 & $\mathrm{CH}_{3} \mathrm{OCOH}$ \\
\hline tEME-AA & $10_{3,7}-9_{2,8}$ & 200605.404 & 31.6 & 3.77 & $\dagger$ & & & \\
\hline tEME-EE & $14_{2,12}-13_{1,13}$ & 200820.096 & 45.3 & 2.62 & $\ldots$ & $\ldots$ & 0.04 & $\mathrm{CH}_{3} \mathrm{OH}$ \\
\hline tEME-EE' & $14_{2,12}-13_{1,13}$ & 200820.098 & 45.3 & 2.62 & $\dagger$ & & & \\
\hline tEME-AE & $14_{2,12}-13_{1,13}$ & 200820.324 & 45.3 & 2.62 & $\grave{\dagger}$ & & & \\
\hline tEME-EA & $14_{2,12}-13_{1,13}$ & 200821.765 & 45.3 & 2.62 & $\ldots$ & $\ldots$ & 0.03 & $\mathrm{CH}_{3} \mathrm{OH}$ \\
\hline tEME-AA & $14_{2,12}-13_{1,13}$ & 200821.991 & 45.3 & 2.62 & $\dagger$ & & & \\
\hline tEME-EE' & $25_{1,25}-24_{0,24}$ & 200871.974 & 124.2 & 19.55 & 200872.2 & 0.59 & 0.20 & $\mathrm{CH}_{2} \mathrm{CHCN} v_{11}=2$ \\
\hline tEME-EE & $25_{1,25}-24_{0,24}$ & 200871.975 & 124.2 & 19.55 & $\doteqdot$ & & & \\
\hline tEME-AE & $25_{1,25}-24_{0,24}$ & 200871.991 & 124.2 & 19.55 & $\dagger$ & & & \\
\hline tEME-EA & $25_{1,25}-24_{0,24}$ & 200872.199 & 124.2 & 19.55 & $\dagger$ & & & \\
\hline tEME-AA & $25_{1,25}-24_{0,24}$ & 200872.216 & 124.2 & 19.55 & $\dagger$ & & & \\
\hline tEME-AA & $26_{0,26}-25_{1,25}$ & 201553.272 & 133.9 & 20.51 & 201553.4 & 0.32 & 0.21 & \\
\hline tEME-EA & $26_{0,26}-25_{1,25}$ & 201553.275 & 133.9 & 20.51 & $\dagger$ & & & \\
\hline tEME-AE & $26_{0,26}-25_{1,25}$ & 201553.416 & 133.9 & 20.51 & $\dagger$ & & & \\
\hline tEME-EE & $26_{0,26}-25_{1,25}$ & 201553.419 & 133.9 & 20.51 & $\dagger$ & & & \\
\hline tEME-EE' & $26_{0,26}-25_{1,25}$ & 201553.419 & 133.9 & 20.51 & $\dot{\dagger}$ & & & \\
\hline tEME-EE' & $19_{2,18}-18_{1,17}$ & 202767.815 & 77.9 & 6.76 & $\ldots$ & $\ldots$ & 0.08 & $\mathrm{H}^{13} \mathrm{CCCN}, \mathrm{CH}_{3} \mathrm{CN} v_{8}=1$ \\
\hline tEME-EE & $19_{2,18}-18_{1,17}$ & 202767.815 & 77.9 & 6.76 & $\dagger$ & & & \\
\hline tEME-AE & $19_{2,18}-18_{1,17}$ & 202767.956 & 77.9 & 6.76 & $\dagger$ & & & \\
\hline tEME-EA & $19_{2,18}-18_{1,17}$ & 202769.267 & 77.9 & 6.76 & $\ldots$ & $\ldots$ & 0.05 & $\mathrm{H}^{13} \mathrm{CCCN}, \mathrm{CH}_{3} \mathrm{CN} v_{8}=1$ \\
\hline tEME-AA & $19_{2,18}-18_{1,17}$ & 202769.407 & 77.9 & 6.76 & $\dagger$ & & & \\
\hline
\end{tabular}


B. Tercero et al.: Trans ethyl methyl ether in space

Table A.2. continued.

\begin{tabular}{|c|c|c|c|c|c|c|c|c|}
\hline Species & $\begin{array}{c}\text { Transition } \\
J_{K_{\mathrm{a}}, K_{\mathrm{c}}}-J_{K_{\mathrm{a}}^{\prime}, K_{\mathrm{c}}^{\prime}}^{\prime}\end{array}$ & $\begin{array}{c}\text { Predicted } \\
\text { frequency }(\mathrm{MHz})\end{array}$ & $\begin{array}{r}E_{\text {upp }} \\
(\mathrm{K})\end{array}$ & $S_{i j}$ & $\begin{array}{c}\text { Observed }^{1} \\
\text { frequency }(\mathrm{MHz})\end{array}$ & $\begin{array}{c}\text { Observed }^{1} \\
T_{\mathrm{MB}}(\mathrm{K})\end{array}$ & $\begin{array}{c}\text { Model }^{2} \\
T_{\mathrm{MB}}(\mathrm{K})\end{array}$ & Blends \\
\hline tEME-AA & $29_{1,28}-28_{2,27}$ & 207138.789 & 170.9 & 12.63 & 207139.6 & 0.07 & 0.07 & \\
\hline tEME-EA & $29_{1,28}-28_{2,27}$ & 207138.838 & 170.9 & 12.63 & $\dagger$ & & & \\
\hline tEME-AE & $29_{1,28}-28_{2,27}$ & 207139.740 & 170.9 & 12.63 & $\dagger$ & & & \\
\hline tEME-EE & $29_{1,28}-28_{2,27}$ & 207139.789 & 170.9 & 12.63 & $\dagger$ & & & \\
\hline tEME-EE' & $29_{1,28}-28_{2,27}$ & 207139.789 & 170.9 & 12.63 & $\dagger$ & & & \\
\hline tEME-EE' & $11_{3,9}-10_{2,8}$ & 207643.771 & 35.9 & 3.90 & $\ldots$ & $\ldots$ & 0.07 & $\mathrm{CH}_{3} \mathrm{CH}_{2} \mathrm{CN}$ \\
\hline tEME-EE & $11_{3,9}-10_{2,8}$ & 207644.152 & 35.9 & 3.94 & $\dagger$ & & & \\
\hline tEME-AE & $11_{3,9}-10_{2,8}$ & 207644.276 & 35.9 & 3.92 & $\dagger$ & & & \\
\hline tEME-EA & $11_{3,9}-10_{2,8}$ & 207647.053 & 35.9 & 3.99 & 207647.4 & 0.20 & 0.05 & $\mathrm{CH}_{3} \mathrm{CH}_{2} \mathrm{CN}$ \\
\hline tEME-AA & $11_{3,9}-10_{2,8}$ & 207647.369 & 35.9 & 3.99 & $\dagger$ & & & \\
\hline tEME-EE' & $5_{4,2}-4_{3,2}$ & 208004.136 & 24.2 & 3.60 & $\ldots$ & $\ldots$ & 0.07 & $\mathrm{CH}_{3} \mathrm{CH}_{2} \mathrm{CN}, \mathrm{CH}_{3} \mathrm{CH}_{2} \mathrm{OH}$ \\
\hline tEME-EE & $5_{4,2}-4_{3,2}$ & 208004.469 & 24.2 & 3.60 & $\dagger$ & & & \\
\hline tEME-AE & $5_{4,2}-4_{3,2}$ & 208004.696 & 24.2 & 3.60 & $\dagger$ & & & \\
\hline tEME-EE & $5_{4,1}-4_{3,1}$ & 208005.368 & 24.2 & 3.60 & $\dagger$ & & & \\
\hline tEME-EE' & $5_{4,1}-4_{3,1}$ & 208005.702 & 24.2 & 3.60 & $\dagger$ & & & \\
\hline tEME-AE & $5_{4,1}-4_{3,1}$ & 208005.929 & 24.2 & 3.60 & $\dagger$ & & & \\
\hline tEME-EA & $5_{4,2}-4_{3,2}$ & 208007.812 & 24.2 & 3.60 & $\ldots$ & $\ldots$ & 0.09 & $\mathrm{CH}_{3} \mathrm{CH}_{2} \mathrm{CN}, \mathrm{CH}_{3} \mathrm{CH}_{2} \mathrm{OH}$ \\
\hline tEME-EA & $5_{4,1}-4_{3,1}$ & 208008.143 & 24.2 & 3.60 & $\dagger$ & & & \\
\hline tEME-AA & $5_{4,2}-4_{3,1}$ & 208008.330 & 24.2 & 3.60 & $\dagger$ & & & \\
\hline tEME-AA & $5_{4,1}-4_{3,2}$ & 208008.413 & 24.2 & 3.60 & $\dagger$ & & & \\
\hline tEME-EE' & $26_{1,26}-25_{0,25}$ & 208147.627 & 134.1 & 20.57 & 208147.7 & 0.58 & 0.22 & $\mathrm{CH}_{2} \mathrm{CHCN} v_{11}=1$ \\
\hline tEME-EE & $26_{1,26}-25_{0,25}$ & 208147.627 & 134.1 & 20.57 & $\dagger$ & & & \\
\hline tEME-AE & $26_{1,26}-25_{0,25}$ & 208147.642 & 134.1 & 20.57 & $\dagger$ & & & \\
\hline tEME-EA & $26_{1,26}-25_{0,25}$ & 208147.831 & 134.1 & 20.57 & $\dagger$ & & & \\
\hline tEME-AA & $26_{1,26}-25_{0,25}$ & 208147.846 & 134.1 & 20.57 & $\dagger$ & & & \\
\hline tEME-EE' & $20_{2,19}-19_{1,18}$ & 208541.474 & 85.6 & 7.24 & 208541.5 & 0.19 & 0.08 & \\
\hline tEME-EE & $20_{2,19}-19_{1,18}$ & 208541.474 & 85.6 & 7.24 & $\dagger$ & & & \\
\hline tEME-AE & $20_{2,19}-19_{1,18}$ & 208541.609 & 85.6 & 7.24 & $\dagger$ & & & \\
\hline tEME-EA & $20_{2,19}-19_{1,18}$ & 208542.901 & 85.6 & 7.24 & 208543.0 & 0.16 & 0.05 & \\
\hline tEME-AA & $20_{2,19}-19_{1,18}$ & 208543.036 & 85.6 & 7.24 & 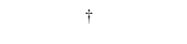 & & & \\
\hline tEME-EE & $11_{3,8}-10_{2,9}$ & 208783.787 & 35.9 & 3.92 & $\ldots$ & $\ldots$ & 0.07 & $\mathrm{CH}_{3} \mathrm{OCOH}$ \\
\hline tEME-EE' & $11_{3,8}-10_{2,9}$ & 208784.168 & 35.9 & 3.88 & $\dagger$ & & & \\
\hline tEME-AE & $11_{3,8}-10_{2,9}$ & 208784.265 & 35.9 & 3.90 & $\dagger$ & & & \\
\hline tEME-EA & $11_{3,8}-10_{2,9}$ & 208785.864 & 35.9 & 3.96 & $\ldots$ & $\ldots$ & 0.05 & $\mathrm{CH}_{3} \mathrm{OCOH}$ \\
\hline tEME-AA & $11_{3,8}-10_{2,9}$ & 208786.150 & 35.9 & 3.96 & $\dagger$ & & & \\
\hline tEME-AA & $27_{0,27}-26_{1,26}$ & 209774.111 & 144.1 & 21.55 & 209774.0 & 0.35 & 0.22 & $\mathrm{CH}_{2} \mathrm{CHCN} v_{15}=1$ \\
\hline tEME-EA & $27_{0,27}-26_{1,26}$ & 209774.113 & 144.1 & 21.55 & $\dagger$ & & & \\
\hline tEME-AE & $27_{0,27}-26_{1,26}$ & 209774.236 & 144.1 & 21.55 & $\dagger$ & & & \\
\hline tEME-EE & $27_{0,27}-26_{1,26}$ & 209774.238 & 144.1 & 21.55 & $\dagger$ & & & \\
\hline tEME-EE' & $27_{0,27}-26_{1,26}$ & 209774.238 & 144.1 & 21.55 & $\dagger$ & & & \\
\hline tEME-EE & $15_{2,13}-14_{1,14}$ & 211933.388 & 51.2 & 2.56 & $\ldots$ & $\ldots$ & 0.04 & $\mathrm{CH}_{3} \mathrm{OCOH}$ \\
\hline tEME-EE' & $15_{2,13}-14_{1,14}$ & 211933.389 & 51.2 & 2.56 & 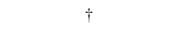 & & & \\
\hline tEME-AE & $15_{2,13}-14_{1,14}$ & 211933.621 & 51.2 & 2.56 & $\dagger$ & & & \\
\hline tEME-EA & $15_{2,13}-14_{1,14}$ & 211935.057 & 51.2 & 2.56 & $\ldots$ & $\ldots$ & 0.03 & $\mathrm{CH}_{3} \mathrm{OCOH}$ \\
\hline tEME-AA & $15_{2,13}-14_{1,14}$ & 211935.290 & 51.2 & 2.56 & $\dagger$ & & & \\
\hline tEME-EE & $32_{5,27}-32_{4,28}$ & 212287.353 & 232.9 & 17.12 & $\ldots$ & $\ldots$ & 0.04 & $\mathrm{CH}_{3} \mathrm{COCH}_{3}$ \\
\hline tEME-EE' & $32_{5,27}-32_{4,28}$ & 212287.576 & 232.9 & 17.07 & $\dagger$ & & & \\
\hline tEME-AE & $32_{5,27}-32_{4,28}$ & 212287.827 & 232.9 & 17.10 & $\dagger$ & & & \\
\hline tEME-EA & $32_{5,27}-32_{4,28}$ & 212290.165 & 232.9 & 17.17 & $\ldots$ & $\ldots$ & 0.03 & $\mathrm{CH}_{3} \mathrm{COCH}_{3}$ \\
\hline tEME-AA & $32_{5,27}-32_{4,28}$ & 212290.528 & 232.9 & 17.17 & $\dagger$ & & & \\
\hline tEME-EE & $31_{5,26}-31_{4,27}$ & 212762.787 & 220.6 & 16.46 & $\ldots$ & $\ldots$ & 0.03 & $\mathrm{CH}_{3} \mathrm{OCH}_{3}$ \\
\hline tEME-EE' & $31_{5,26}-31_{4,27}$ & 212763.095 & 220.6 & 16.37 & $\dagger$ & & & \\
\hline tEME-AE & $31_{5,26}-31_{4,27}$ & 212763.306 & 220.6 & 16.42 & $\dagger$ & & & \\
\hline tEME-EA & $31_{5,26}-31_{4,27}$ & 212765.533 & 220.6 & 16.55 & 212766.9 & 0.14 & 0.03 & $\mathrm{CH}_{3} \mathrm{OCH}_{3}$ \\
\hline tEME-AA & $31_{5,26}-31_{4,27}$ & 212765.897 & 220.6 & 16.55 & $\dagger$ & & & \\
\hline tEME-EE & $30_{5,25}-30_{4,26}$ & 213178.397 & 208.6 & 15.77 & $\ldots$ & $\ldots$ & 0.04 & U-line \\
\hline tEME-EE' & $30_{5,25}-30_{4,26}$ & 213178.825 & 208.6 & 15.61 & $\dagger$ & & & \\
\hline tEME-AE & $30_{5,25}-30_{4,26}$ & 213178.976 & 208.6 & 15.70 & $\dagger$ & & & \\
\hline tEME-EA & $30_{5,25}-30_{4,26}$ & 213181.046 & 208.6 & 15.93 & 213181.4 & 0.21 & 0.03 & $\mathrm{CH}_{2} \mathrm{OHCHO}$ \\
\hline tEME-AA & $30_{5,25}-30_{4,26}$ & 213181.410 & 208.6 & 15.94 & $\dagger$ & & & \\
\hline tEME-EE' & $33_{5,29}-33_{4,30}$ & 213495.962 & 245.7 & 17.69 & 213495.8 & 0.09 & 0.04 & U-line \\
\hline tEME-EE & $33_{5,29}-33_{4,30}$ & 213496.124 & 245.7 & 17.72 & $\dagger$ & & & \\
\hline tEME-AE & $33_{5,29}-33_{4,30}$ & 213496.418 & 245.7 & 17.71 & $\dagger$ & & & \\
\hline tEME-EA & $33_{5,29}-33_{4,30}$ & 213499.211 & 245.7 & 17.75 & 213499.3 & 0.10 & 0.03 & U-line \\
\hline tEME-AA & $33_{5,29}-33_{4,30}$ & 213499.587 & 245.7 & 17.75 & $\dagger$ & & & \\
\hline tEME-EE & $29_{5,24}-29_{4,25}$ & 213540.983 & 196.9 & 15.03 & 213541.4 & 0.16 & 0.05 & $\mathrm{CH}_{2} \mathrm{CHCN} v_{11}=2$ \\
\hline tEME-EE' & $29_{5,24}-29_{4,25}$ & 213541.573 & 196.9 & 14.74 & 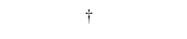 & & & \\
\hline tEME-AE & $29_{5,24}-29_{4,25}$ & 213541.643 & 196.9 & 14.89 & $\dagger$ & & & \\
\hline tEME-EA & $29_{5,24}-29_{4,25}$ & 213543.491 & 196.9 & 15.32 & $\ldots$ & $\ldots$ & 0.04 & $\mathrm{CH}_{2} \mathrm{CHCN} v_{11}=2$ \\
\hline tEME-AA & $29_{5,24}-29_{4,25}$ & 213543.850 & 196.9 & 15.33 & $\dot{\dagger}$ & & & \\
\hline tEME-EE' & $32_{5,28}-32_{4,29}$ & 213676.308 & 232.9 & 17.03 & 213676.5 & 0.20 & 0.04 & $\mathrm{CH}_{3} \mathrm{CH}_{2} \mathrm{OH}$ \\
\hline tEME-EE & $32_{5,28}-32_{4,29}$ & 213676.530 & 232.9 & 17.09 & $\dot{\dagger}$ & & & \\
\hline tEME-AE & $32_{5,28}-32_{4,29}$ & 213676.802 & 232.9 & 17.06 & $\dagger$ & & & \\
\hline
\end{tabular}


A\&A 582, L1 (2015)

Table A.2. continued.

\begin{tabular}{|c|c|c|c|c|c|c|c|c|}
\hline Species & $\begin{array}{c}\text { Transition } \\
J_{K_{\mathrm{a}}, K_{\mathrm{c}}}-J_{K_{\mathrm{a}}^{\prime}, K_{\mathrm{c}}^{\prime}}^{\prime}\end{array}$ & $\begin{array}{c}\text { Predicted } \\
\text { frequency }(\mathrm{MHz})\end{array}$ & $\begin{array}{r}E_{\text {upp }} \\
(\mathrm{K})\end{array}$ & $S_{i j}$ & $\begin{array}{c}\text { Observed }^{1} \\
\text { frequency }(\mathrm{MHz})\end{array}$ & $\begin{array}{c}\text { Observed }^{1} \\
T_{\mathrm{MB}}(\mathrm{K})\end{array}$ & $\begin{array}{c}\text { Model }^{2} \\
T_{\mathrm{MB}}(\mathrm{K})\end{array}$ & Blends \\
\hline tEME-EA & $32_{5,28}-32_{4,29}$ & 213679.692 & 232.9 & 17.13 & 213680.0 & 0.14 & 0.03 & $\mathrm{CH}_{3} \mathrm{COOCH}_{3}$ \\
\hline tEME-AA & $32_{5,28}-32_{4,29}$ & 213680.076 & 232.9 & 17.14 & $\dagger$ & & & \\
\hline tEME-EE' & $31_{5,27}-31_{4,28}$ & 213854.674 & 220.5 & 16.34 & $\ldots$ & $\ldots$ & 0.05 & $\mathrm{CH}_{3} \mathrm{CH}_{2} \mathrm{OH}$ \\
\hline tEME-EE & $31_{5,27}-31_{4,28}$ & 213854.982 & 220.5 & 16.44 & $\dagger$ & & & \\
\hline tEME-AE & $31_{5,27}-31_{4,28}$ & 213855.220 & 220.5 & 16.39 & $\dagger$ & & & \\
\hline tEME-EE & $28_{5,23}-28_{4,24}$ & 213856.708 & 185.7 & 14.18 & $\ldots$ & $\ldots$ & 0.05 & $\mathrm{CH}_{3} \mathrm{CH}_{2} \mathrm{OH}$ \\
\hline tEME-AE & $28_{5,23}-28_{4,24}$ & 213857.473 & 185.7 & 13.94 & $\dagger$ & & & \\
\hline tEME-EE' & $28_{5,23}-28_{4,24}$ & 213857.509 & 185.7 & 13.70 & $\dot{\dagger}$ & & & \\
\hline tEME-EA & $31_{5,27}-31_{4,28}$ & 213858.239 & 220.5 & 16.52 & $\ldots$ & $\ldots$ & 0.05 & $\mathrm{CH}_{3} \mathrm{CH}_{2} \mathrm{OH}$ \\
\hline tEME-AA & $31_{5,27}-31_{4,28}$ & 213858.632 & 220.5 & 16.53 & $\dddot{\dagger}$ & & & \\
\hline tEME-EA & $28_{5,23}-28_{4,24}$ & 213859.015 & 185.7 & 14.70 & $\dagger$ & & & \\
\hline tEME-AA & $28_{5,23}-28_{4,24}$ & 213859.367 & 185.7 & 14.73 & $\dagger$ & & & \\
\hline tEME-EE' & $30_{5,26}-30_{4,27}$ & 214028.729 & 208.6 & 15.59 & $\ldots$ & $\ldots$ & 0.05 & $\mathrm{CH}_{3} \mathrm{COCH}_{3}$ \\
\hline tEME-EE & $30_{5,26}-30_{4,27}$ & 214029.157 & 208.6 & 15.76 & $\dagger$ & & & \\
\hline tEME-AE & $30_{5,26}-30_{4,27}$ & 214029.345 & 208.6 & 15.68 & $\dagger$ & & & \\
\hline tEME-EA & $30_{5,26}-30_{4,27}$ & 214032.539 & 208.6 & 15.91 & $\ldots$ & $\ldots$ & 0.04 & \\
\hline tEME-AA & $30_{5,26}-30_{4,27}$ & 214032.943 & 208.6 & 15.92 & $\dddot{\dagger}$ & & & \\
\hline tEME-EE & $27_{5,22}-27_{4,23}$ & 214131.118 & 174.9 & 13.15 & $\ldots$ & $\ldots$ & 0.04 & $\mathrm{CH}_{3} \mathrm{CH}_{2}{ }^{13} \mathrm{CN}, \mathrm{CH}_{3} \mathrm{COOH} v_{t}=1$ \\
\hline tEME-AE & $27_{5,22}-27_{4,23}$ & 214132.010 & 174.9 & 12.80 & 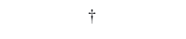 & & & \\
\hline tEME-EE' & $27_{5,22}-27_{4,23}$ & 214132.166 & 174.9 & 12.45 & $\dagger$ & & & \\
\hline tEME-EA & $27_{5,22}-27_{4,23}$ & 214133.150 & 174.9 & 14.07 & $\ldots$ & $\ldots$ & 0.04 & $\mathrm{CH}_{3} \mathrm{CH}_{2}{ }^{13} \mathrm{CN}, \mathrm{CH}_{3} \mathrm{COOH} v_{t}=1$ \\
\hline tEME-AA & $27_{5,22}-27_{4,23}$ & 214133.486 & 174.9 & 14.14 & $\dagger$ & & & \\
\hline tEME-EE' & $29_{5,25}-29_{4,25}$ & 214196.531 & 196.9 & 14.72 & $\ldots$ & $\ldots$ & 0.05 & $\mathrm{CH}_{3}{ }^{13} \mathrm{CH}_{2} \mathrm{CN}, \mathrm{CH}_{3} \mathrm{O}^{13} \mathrm{COH} v_{t}=1$ \\
\hline tEME-EE & $29_{5,25}-29_{4,25}$ & 214197.122 & 196.9 & 15.02 & $\dot{\dagger}$ & & & \\
\hline tEME-AE & $29_{5,25}-29_{4,25}$ & 214197.239 & 196.9 & 14.88 & $\dagger$ & & & \\
\hline tEME-EA & $29_{5,25}-29_{4,25}$ & 214200.673 & 196.9 & 15.30 & $\ldots$ & $\ldots$ & 0.04 & $\mathrm{CH}_{3}{ }^{13} \mathrm{CH}_{2} \mathrm{CN}, \mathrm{CH}_{3} \mathrm{O}^{13} \mathrm{COH} v_{t}=1$ \\
\hline tEME-AA & $29_{5,25}-29_{4,25}$ & 214201.091 & 196.9 & 15.32 & $\dagger$ & & & \\
\hline tEME-EE' & $21_{2,20}-20_{1,19}$ & 214246.202 & 93.7 & 7.76 & 214246.2 & 0.15 & 0.09 & ${ }^{13} \mathrm{CH}_{3} \mathrm{CN}$ \\
\hline tEME-EE & $21_{2,20}-20_{1,19}$ & 214246.202 & 93.7 & 7.76 & $\dagger$ & & & \\
\hline tEME-AE & $21_{2,20}-20_{1,19}$ & 214246.332 & 93.7 & 7.76 & $\dagger$ & & & \\
\hline tEME-EA & $21_{2,20}-20_{1,19}$ & 214247.602 & 93.7 & 7.76 & 214247.7 & 0.11 & 0.06 & ${ }^{13} \mathrm{CH}_{3} \mathrm{CN}$ \\
\hline tEME-AA & $21_{2,20}-20_{1,19}$ & 214247.732 & 93.7 & 7.76 & $\doteqdot$ & & & \\
\hline tEME-EE' & $26_{5,22}-26_{4,22}$ & 214355.828 & 164.4 & 2.47 & $\ldots$ & $\ldots$ & 0.05 & $\mathrm{SO},{ }^{13} \mathrm{CH}_{3} \mathrm{CN}$ \\
\hline tEME-EE' & $28_{5,24}-28_{4,25}$ & 214356.510 & 185.7 & 13.69 & $\dagger$ & & & \\
\hline tEME-AE & $26_{5,22}-26_{4,22}$ & 214356.963 & 164.4 & 2.08 & $\dagger$ & & & \\
\hline tEME-EE & $26_{5,22}-26_{4,22}$ & 214357.232 & 164.4 & 1.63 & $\dagger$ & & & \\
\hline tEME-EE & $28_{5,24}-28_{4,25}$ & 214357.312 & 185.7 & 14.17 & $\dagger$ & & & \\
\hline tEME-AE & $28_{5,24}-28_{4,25}$ & 214357.333 & 185.7 & 13.93 & $\dagger$ & & & \\
\hline tEME-EA & $28_{5,24}-28_{4,25}$ & 214361.091 & 185.7 & 14.69 & $\ldots$ & $\ldots$ & 0.04 & $\mathrm{SO},{ }^{13} \mathrm{CH}_{3} \mathrm{CN}$ \\
\hline tEME-AA & $28_{5,24}-28_{4,25}$ & 214361.527 & 185.7 & 14.72 & $\dddot{\dagger}$ & & & \\
\hline tEME-EE & $26_{5,21}-26_{4,22}$ & 214369.161 & 164.4 & 11.92 & $\ldots$ & $\ldots$ & 0.07 & $\mathrm{SO},{ }^{13} \mathrm{CH}_{3} \mathrm{CN}$ \\
\hline tEME-AE & $26_{5,21}-26_{4,22}$ & 214370.185 & 164.4 & 11.47 & 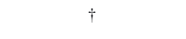 & & & \\
\hline tEME-EE' & $26_{5,21}-26_{4,22}$ & 214370.456 & 164.4 & 11.08 & $\dagger$ & & & \\
\hline tEME-EA & $26_{5,21}-26_{4,22}$ & 214370.843 & 164.4 & 13.42 & $\dagger$ & & & \\
\hline tEME-AA & $26_{5,21}-26_{4,22}$ & 214371.154 & 164.4 & 13.55 & $\dagger$ & & & \\
\hline tEME-EE' & $27_{5,23}-27_{4,24}$ & 214507.462 & 174.9 & 12.45 & $\ldots$ & $\ldots$ & 0.04 & $\mathrm{CH}_{3} \mathrm{CH}_{2} \mathrm{CN}$ \\
\hline tEME-AE & $27_{5,23}-27_{4,24}$ & 214508.414 & 174.9 & 12.79 & $\dddot{\dagger}$ & & & \\
\hline tEME-EE & $27_{5,23}-27_{4,24}$ & 214508.510 & 174.9 & 13.15 & $\dagger$ & & & \\
\hline tEME-EA & $27_{5,23}-27_{4,24}$ & 214512.591 & 174.9 & 14.07 & $\ldots$ & $\ldots$ & 0.04 & $\mathrm{CH}_{3} \mathrm{CH}_{2} \mathrm{CN}$ \\
\hline tEME-AA & $27_{5,23}-27_{4,24}$ & 214513.051 & 174.9 & 14.13 & $\grave{\dagger}$ & & & \\
\hline tEME-EE & $25_{5,20}-25_{4,21}$ & 214575.213 & 154.4 & 10.55 & $\ldots$ & $\ldots$ & 0.09 & ${ }^{13} \mathrm{C}^{17} \mathrm{O}$ \\
\hline tEME-AE & $25_{5,20}-25_{4,21}$ & 214576.349 & 154.4 & 10.10 & $\dagger$ & & & \\
\hline tEME-EA & $25_{5,20}-25_{4,21}$ & 214576.498 & 154.4 & 12.69 & $\dagger$ & & & \\
\hline tEME-EE' & $25_{5,20}-25_{4,21}$ & 214576.706 & 154.4 & 9.74 & $\dagger$ & & & \\
\hline tEME-AA & $25_{5,20}-25_{4,21}$ & 214576.769 & 154.4 & 12.96 & $\dagger$ & & & \\
\hline tEME-EE' & $26_{5,22}-26_{4,23}$ & 214648.535 & 164.4 & 11.08 & $\ldots$ & $\ldots$ & 0.04 & $\mathrm{CH}_{3} \mathrm{OCOH}$ \\
\hline tEME-AE & $26_{5,22}-26_{4,23}$ & 214649.611 & 164.4 & 11.47 & $\dddot{\dagger}$ & & & \\
\hline tEME-EE & $26_{5,22}-26_{4,23}$ & 214649.829 & 164.4 & 11.92 & $\dagger$ & & & \\
\hline tEME-EA & $26_{5,22}-26_{4,23}$ & 214654.288 & 164.5 & 13.41 & $\ldots$ & $\ldots$ & 0.04 & $\mathrm{CH}_{3} \mathrm{OCOH}$ \\
\hline tEME-AA & $26_{5,22}-26_{4,23}$ & 214654.781 & 164.5 & 13.54 & $\dagger$ & & & \\
\hline tEME-EE & $24_{5,19}-24_{4,20}$ & 214753.118 & 144.7 & 9.23 & 214754.3 & 0.20 & 0.09 & \\
\hline tEME-EA & $24_{5,19}-24_{4,20}$ & 214754.023 & 144.7 & 11.84 & $\dagger$ & & & \\
\hline tEME-AA & $24_{5,19}-24_{4,20}$ & 214754.233 & 144.7 & 12.38 & $\dagger$ & & & \\
\hline tEME-AE & $24_{5,19}-24_{4,20}$ & 214754.329 & 144.7 & 8.86 & $\dagger$ & & & \\
\hline tEME-EE' & $24_{5,19}-24_{4,20}$ & 214754.733 & 144.7 & 8.60 & $\dagger$ & & & \\
\hline tEME-EE' & $25_{5,21}-25_{4,22}$ & 214779.208 & 154.4 & 9.74 & $\ldots$ & $\ldots$ & 0.04 & $\mathrm{CH}_{3} \mathrm{OCOH}$ \\
\hline tEME-AE & $25_{5,21}-25_{4,22}$ & 214780.378 & 154.4 & 10.10 & $\dagger$ & & & \\
\hline tEME-EE & $25_{5,21}-25_{4,22}$ & 214780.701 & 154.4 & 10.55 & $\dagger$ & & & \\
\hline tEME-EA & $25_{5,21}-25_{4,22}$ & 214785.581 & 154.4 & 12.69 & $\ldots$ & $\ldots$ & 0.04 & $\mathrm{CH}_{3} \mathrm{OCOH}$ \\
\hline tEME-AA & $25_{5,21}-25_{4,22}$ & 214786.123 & 154.4 & 12.96 & $\dot{\dagger}$ & & & \\
\hline tEME-EE' & $24_{5,20}-24_{4,21}$ & 214899.228 & 144.7 & 8.61 & $\ldots$ & $\ldots$ & 0.04 & $\mathrm{CH}_{3} \mathrm{OCOH} v_{t}=1$ \\
\hline tEME-AE & $24_{5,20}-24_{4,21}$ & 214900.454 & 144.7 & 8.87 & $\dddot{\dagger}$ & & & \\
\hline tEME-EE & $24_{5,20}-24_{4,21}$ & 214900.844 & 144.7 & 9.23 & $\dagger$ & & & \\
\hline
\end{tabular}


B. Tercero et al.: Trans ethyl methyl ether in space

Table A.2. continued.

\begin{tabular}{|c|c|c|c|c|c|c|c|c|}
\hline Species & $\begin{array}{c}\text { Transition } \\
J_{K_{\mathrm{a}}, K_{\mathrm{c}}}-J_{K_{\mathrm{a}}^{\prime}, K_{\mathrm{c}}^{\prime}}^{\prime}\end{array}$ & $\begin{array}{c}\text { Predicted } \\
\text { frequency }(\mathrm{MHz})\end{array}$ & $\begin{array}{r}E_{\text {upp }} \\
(\mathrm{K})\end{array}$ & $S_{i j}$ & $\begin{array}{c}\text { Observed }^{1} \\
\text { frequency }(\mathrm{MHz})\end{array}$ & $\begin{array}{c}\text { Observed }^{1} \\
T_{\mathrm{MB}}(\mathrm{K})\end{array}$ & $\begin{array}{c}\text { Model }^{2} \\
T_{\mathrm{MB}}(\mathrm{K})\end{array}$ & Blends \\
\hline tEME-EA & $24_{5,20}-24_{4,21}$ & 214906.128 & 144.7 & 11.83 & $\ldots$ & $\ldots$ & 0.11 & $\mathrm{CH}_{3} \mathrm{CH}_{2} \mathrm{CN}$ \\
\hline tEME-EE & $23_{5,18}-23_{4,19}$ & 214906.260 & 135.4 & 8.13 & $\dagger$ & & & \\
\hline tEME-AA & $24_{5,20}-24_{4,21}$ & 214906.739 & 144.7 & 12.38 & $\dagger$ & & & \\
\hline tEME-EA & $23_{5,18}-23_{4,19}$ & 214906.872 & 135.4 & 10.76 & $\dagger$ & & & \\
\hline tEME-AA & $23_{5,18}-23_{4,19}$ & 214906.992 & 135.4 & 11.80 & $\dagger$ & & & \\
\hline tEME-AE & $23_{5,18}-23_{4,19}$ & 214907.507 & 135.4 & 7.91 & $\dagger$ & & & \\
\hline tEME-EE' & $23_{5,18}-23_{4,19}$ & 214907.924 & 135.4 & 7.77 & $\dagger$ & & & \\
\hline tEME-EE & $24_{5,19}-24_{4,21}$ & 214909.858 & 144.7 & 3.15 & $\dagger$ & & & \\
\hline tEME-EA & $24_{5,19}-24_{4,21}$ & 214910.548 & 144.7 & 0.55 & $\dagger$ & & & \\
\hline tEME-AE & $24_{5,19}-24_{4,21}$ & 214911.166 & 144.7 & 3.51 & $\dagger$ & & & \\
\hline tEME-EE' & $24_{5,19}-24_{4,21}$ & 214911.685 & 144.7 & 3.77 & $\dagger$ & & & \\
\hline tEME-EE' & $23_{5,19}-23_{4,20}$ & 215008.545 & 135.4 & 7.77 & $\ldots$ & $\ldots$ & 0.02 & $\mathrm{CH}_{3} \mathrm{CH}_{2} \mathrm{CN} v_{13} / v_{21}$ \\
\hline tEME-AE & $23_{5,19}-23_{4,20}$ & 215009.791 & 135.4 & 7.91 & $\ldots$ & $\ldots$ & 0.04 & $\mathrm{CH}_{3} \mathrm{CH}_{2} \mathrm{CN} v_{13} / v_{21}$ \\
\hline tEME-EE & $23_{5,19}-23_{4,20}$ & 215010.209 & 135.4 & 8.13 & $\dagger$ & & & \\
\hline tEME-EA & $23_{5,19}-23_{4,20}$ & 215015.810 & 135.4 & 10.75 & 215016.7 & 0.05 & 0.04 & \\
\hline tEME-AA & $23_{5,19}-23_{4,20}$ & 215016.519 & 135.4 & 11.80 & $\dagger$ & & & \\
\hline tEME-EA & $22_{5,18}-22_{4,18}$ & 215035.544 & 126.6 & 1.78 & $\ldots$ & $\ldots$ & 0.07 & $\mathrm{CH}_{3} \mathrm{CH}_{2} \mathrm{CN}$ \\
\hline tEME-EE & $22_{5,17}-22_{4,18}$ & 215037.639 & 126.6 & 7.33 & $\dagger$ & & & \\
\hline tEME-EA & $22_{5,17}-22_{4,18}$ & 215038.085 & 126.6 & 9.45 & $\dagger$ & & & \\
\hline tEME-AA & $22_{5,17}-22_{4,18}$ & 215038.089 & 126.6 & 11.23 & $\dagger$ & & & \\
\hline tEME-AE & $22_{5,17}-22_{4,18}$ & 215038.890 & 126.6 & 7.24 & $\dagger$ & & & \\
\hline tEME-EE' & $22_{5,17}-22_{4,18}$ & 215039.292 & 126.6 & 7.21 & $\dagger$ & & & \\
\hline tEME-AA & $35_{2,33}-34_{3,32}$ & 215107.148 & 250.8 & 9.27 & $\ldots$ & $\ldots$ & 0.03 & $\mathrm{CH}_{3} \mathrm{CH}_{2} \mathrm{CN}$ \\
\hline tEME-EA & $35_{2,33}-34_{3,32}$ & 215107.270 & 250.8 & 9.27 & $\dagger$ & & & \\
\hline tEME-EE' & $22_{5,18}-22_{4,19}$ & 215107.270 & 126.6 & 7.21 & $\dagger$ & & & \\
\hline tEME-AE & $22_{5,18}-22_{4,19}$ & 215108.508 & 126.6 & 7.24 & $\dagger$ & & & \\
\hline tEME-EE & $22_{5,18}-22_{4,19}$ & 215108.923 & 126.6 & 7.33 & $\ldots$ & $\ldots$ & 0.05 & $\mathrm{CH}_{3} \mathrm{CH}_{2} \mathrm{CN}$ \\
\hline tEME-AE & $35_{2,33}-34_{3,32}$ & 215109.184 & 250.8 & 9.27 & $\dagger$ & & & \\
\hline tEME-EE & $35_{2,33}-34_{3,32}$ & 215109.306 & 250.8 & 9.27 & $\dagger$ & & & \\
\hline tEME-EE' & $35_{2,33}-34_{3,32}$ & 215109.306 & 250.8 & 9.27 & $\dagger$ & & & \\
\hline tEME-EA & $22_{5,18}-22_{4,19}$ & 215114.713 & 126.6 & 9.45 & $\ldots$ & $\ldots$ & 0.04 & $\mathrm{CH}_{3} \mathrm{CH}_{2} \mathrm{CN}$ \\
\hline tEME-AA & $22_{5,18}-22_{4,19}$ & 215115.545 & 126.6 & 11.23 & $\dagger$ & & & \\
\hline tEME-EE & $22_{5,17}-22_{4,19}$ & 215117.232 & 126.6 & 3.90 & $\dagger$ & & & \\
\hline tEME-EA & $22_{5,17}-22_{4,19}$ & 215117.254 & 126.6 & 1.78 & $\dagger$ & & & \\
\hline tEME-AE & $22_{5,17}-22_{4,19}$ & 215118.679 & 126.6 & 3.99 & $\dagger$ & & & \\
\hline tEME-EE' & $22_{5,17}-22_{4,19}$ & 215119.312 & 126.6 & 4.02 & $\dagger$ & & & \\
\hline tEME-EA & $21_{5,17}-21_{4,17}$ & 215148.139 & 118.0 & 2.58 & 215150.3 & 0.25 & 0.07 & $\mathrm{CH}_{3} \mathrm{COCH}_{3}$ \\
\hline tEME-EE & $21_{5,16}-21_{4,17}$ & 215149.914 & 118.0 & 6.81 & $\dagger$ & & & \\
\hline tEME-AA & $21_{5,16}-21_{4,17}$ & 215150.202 & 118.0 & 10.66 & $\dagger$ & & & \\
\hline tEME-EA & $21_{5,16}-21_{4,17}$ & 215150.327 & 118.0 & 8.07 & $\dagger$ & & & \\
\hline tEME-AE & $21_{5,16}-21_{4,17}$ & 215151.142 & 118.0 & 6.84 & $\dagger$ & & & \\
\hline tEME-EE' & $21_{5,16}-21_{4,17}$ & 215151.503 & 118.0 & 6.91 & $\dagger$ & & & \\
\hline tEME-EE' & $21_{5,17}-21_{4,18}$ & 215195.665 & 118.0 & 6.91 & $\ldots$ & $\ldots$ & 0.04 & SO \\
\hline tEME-AE & $21_{5,17}-21_{4,18}$ & 215196.869 & 118.0 & 6.84 & $\dagger$ & & & \\
\hline tEME-EE & $21_{5,17}-21_{4,18}$ & 215197.254 & 118.0 & 6.81 & $\dagger$ & & & \\
\hline tEME-EA & $21_{5,17}-21_{4,18}$ & 215203.100 & 118.0 & 8.07 & $\ldots$ & $\ldots$ & 0.04 & SO \\
\hline tEME-AA & $21_{5,17}-21_{4,18}$ & 215204.068 & 118.0 & 10.66 & $\dagger$ & & & \\
\hline tEME-EA & $21_{5,16}-21_{4,18}$ & 215205.287 & 118.0 & 2.58 & $\dagger$ & & & \\
\hline tEME-EE & $21_{5,16}-21_{4,18}$ & 215205.486 & 118.0 & 3.85 & $\dagger$ & & & \\
\hline tEME-EA & $20_{5,16}-20_{4,16}$ & 215243.901 & 109.9 & 3.19 & $\ldots$ & $\ldots$ & 0.07 & SO \\
\hline tEME-EE & $20_{5,15}-20_{4,16}$ & 215245.438 & 109.9 & 6.54 & $\dagger$ & & & \\
\hline tEME-AA & $20_{5,15}-20_{4,16}$ & 215245.691 & 109.9 & 10.09 & $\dagger$ & & & \\
\hline tEME-EA & $20_{5,15}-20_{4,16}$ & 215245.934 & 109.9 & 6.89 & $\dagger$ & & & \\
\hline tEME-AE & $20_{5,15}-20_{4,16}$ & 215246.615 & 109.9 & 6.68 & $\dagger$ & & & \\
\hline tEME-EE' & $20_{5,15}-20_{4,16}$ & 215246.907 & 109.9 & 6.83 & $\dagger$ & & & \\
\hline tEME-EE' & $20_{5,16}-20_{4,17}$ & 215274.126 & 109.9 & 6.83 & $\ldots$ & $\ldots$ & 0.04 & $\mathrm{CH}_{3} \mathrm{CH}_{2} \mathrm{CN} v_{20}=1$ \\
\hline tEME-AE & $20_{5,16}-20_{4,17}$ & 215275.268 & 109.9 & 6.68 & $\dagger$ & & & \\
\hline tEME-EE & $20_{5,16}-20_{4,17}$ & 215275.595 & 109.9 & 6.54 & $\dagger$ & & & \\
\hline tEME-EA & $20_{5,16}-20_{4,17}$ & 215281.378 & 109.9 & 6.89 & $\ldots$ & $\ldots$ & 0.04 & $\mathrm{CH}_{3} \mathrm{CH}_{2} \mathrm{CN} v_{13} / v_{21}$ \\
\hline tEME-AA & $20_{5,16}-20_{4,17}$ & 215282.470 & 109.9 & 10.09 & $\dagger$ & & & \\
\hline tEME-EA & $20_{5,15}-20_{4,17}$ & 215283.411 & 109.9 & 3.19 & $\dagger$ & & & \\
\hline tEME-EE & $20_{5,15}-20_{4,17}$ & 215283.810 & 109.9 & 3.55 & $\dagger$ & & & \\
\hline tEME-EA & $19_{5,15}-19_{4,15}$ & 215324.992 & 102.2 & 3.49 & 215326.9 & 0.20 & 0.08 & $\mathrm{CH}_{3} \mathrm{OCOH}$ \\
\hline tEME-EE & $19_{5,14}-19_{4,15}$ & 215326.280 & 102.2 & 6.50 & $\doteqdot$ & & & \\
\hline tEME-AA & $19_{5,14}-19_{4,15}$ & 215326.637 & 102.2 & 9.52 & $\dagger$ & & & \\
\hline tEME-EA & $19_{5,14}-19_{4,15}$ & 215326.971 & 102.2 & 6.03 & $\dagger$ & & & \\
\hline tEME-AE & $19_{5,14}-19_{4,15}$ & 215327.373 & 102.2 & 6.72 & $\dagger$ & & & \\
\hline tEME-EE' & $19_{5,14}-19_{4,15}$ & 215327.561 & 102.2 & 6.95 & $\dagger$ & & & \\
\hline tEME-EE' & $19_{5,15}-19_{4,16}$ & 215343.169 & 102.2 & 6.95 & 215344.3 & 0.20 & 0.05 & $\mathrm{CH}_{3} \mathrm{OCOH} v_{t}=1$ \\
\hline tEME-AE & $19_{5,15}-19_{4,16}$ & 215344.214 & 102.2 & 6.72 & $\dot{\dagger}$ & & & \\
\hline tEME-EE & $19_{5,15}-19_{4,16}$ & 215344.450 & 102.2 & 6.50 & $\dagger$ & & & \\
\hline tEME-EA & $19_{5,15}-19_{4,16}$ & 215350.059 & 102.2 & 6.03 & $\ldots$ & $\ldots$ & 0.05 & $\mathrm{CH}_{3} \mathrm{CH}_{2} \mathrm{CN}$ \\
\hline tEME-AA & $19_{5,15}-19_{4,16}$ & 215351.248 & 102.2 & 9.52 & $\dagger$ & & & \\
\hline
\end{tabular}


A\&A 582, L1 (2015)

Table A.2. continued.

\begin{tabular}{|c|c|c|c|c|c|c|c|c|}
\hline Species & $\begin{array}{c}\text { Transition } \\
J_{K_{\mathrm{a}}, K_{\mathrm{c}}}-J_{K_{\mathrm{a}}^{\prime}, K_{\mathrm{c}}^{\prime}}^{\prime}\end{array}$ & $\begin{array}{c}\text { Predicted } \\
\text { frequency }(\mathrm{MHz})\end{array}$ & $\begin{array}{r}E_{\text {upp }} \\
(\mathrm{K})\end{array}$ & $S_{i j}$ & $\begin{array}{c}\text { Observed }^{1} \\
\text { frequency }(\mathrm{MHz})\end{array}$ & $\begin{array}{c}\text { Observed }^{1} \\
T_{\mathrm{MB}}(\mathrm{K})\end{array}$ & $\begin{array}{c}\text { Model }^{2} \\
T_{\mathrm{MB}}(\mathrm{K})\end{array}$ & Blends \\
\hline tEME-EE' & $12_{3,10}-11_{2,9}$ & 215361.252 & 40.5 & 4.16 & $\ldots$ & $\ldots$ & 0.07 & $\mathrm{CH}_{3} \mathrm{CH}_{2} \mathrm{CN} v_{13} / v_{21}$ \\
\hline tEME-EE & $12_{3,10}-11_{2,9}$ & 215361.484 & 40.5 & 4.18 & 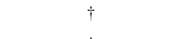 & & & \\
\hline tEME-AE & $12_{3,10}-11_{2,9}$ & 215361.676 & 40.5 & 4.17 & $\dagger$ & & & \\
\hline tEME-EA & $12_{3,10}-11_{2,9}$ & 215364.224 & 40.5 & 4.19 & $\ldots$ & $\ldots$ & 0.05 & $\mathrm{CH}_{3} \mathrm{CH}_{2} \mathrm{CN} v_{13} / v_{21}$ \\
\hline tEME-AA & $12_{3,10}-11_{2,9}$ & 215364.533 & 40.5 & 4.20 & $\doteqdot$ & & & \\
\hline tEME-EE & $18_{5,13}-18_{4,14}$ & 215394.256 & 94.8 & 6.65 & $\ldots$ & $\ldots$ & 0.11 & $\mathrm{CH}_{3} \mathrm{CH}_{2} \mathrm{CN}$ \\
\hline tEME-AA & $18_{5,13}-18_{4,14}$ & 215394.875 & 94.8 & 8.95 & $\grave{\dagger}$ & & & \\
\hline tEME-AE & $18_{5,13}-18_{4,14}$ & 215395.227 & 94.8 & 6.94 & $\dagger$ & & & \\
\hline tEME-EA & $18_{5,13}-18_{4,14}$ & 215395.264 & 94.8 & 5.47 & $\dagger$ & & & \\
\hline tEME-EE' & $18_{5,13}-18_{4,14}$ & 215395.280 & 94.8 & 7.19 & $\dagger$ & & & \\
\hline tEME-EE' & $18_{5,14}-18_{4,15}$ & 215403.394 & 94.8 & 7.19 & $\ldots$ & $\ldots$ & 0.06 & $\mathrm{CH}_{3} \mathrm{CH}_{2} \mathrm{CN}$ \\
\hline tEME-AE & $18_{5,14}-18_{4,15}$ & 215404.308 & 94.8 & 6.94 & $\dot{\dagger}$ & & & \\
\hline tEME-EE & $18_{5,14}-18_{4,15}$ & 215404.417 & 94.8 & 6.65 & $\dagger$ & & & \\
\hline tEME-EA & $18_{5,14}-18_{4,15}$ & 215409.728 & 94.8 & 5.47 & $\ldots$ & $\ldots$ & 0.09 & $\mathrm{CH}_{3} \mathrm{CH}_{2} \mathrm{CN} v=0 ; v_{13} / v_{21}$ \\
\hline tEME-AA & $18_{5,14}-18_{4,15}$ & 215410.979 & 94.8 & 8.95 & $\doteqdot$ & & & \\
\hline tEME-EA & $17_{5,13}-17_{4,13}$ & 215450.467 & 87.9 & 3.22 & 215451.8 & 0.47 & 0.11 & $\mathrm{CH}_{3} \mathrm{CH}_{2} \mathrm{CN} v_{20}=1, \mathrm{NH}_{2} \mathrm{CHO} v_{12}=1$ \\
\hline tEME-EE & $17_{5,13}-17_{4,13}$ & 215450.974 & 87.9 & 6.90 & $\dot{\dagger}$ & & & \\
\hline tEME-EE' & $17_{5,13}-17_{4,13}$ & 215451.703 & 87.9 & 7.39 & $\dagger$ & & & \\
\hline tEME-AE & $17_{5,13}-17_{4,13}$ & 215451.799 & 87.9 & 7.18 & $\dagger$ & & & \\
\hline tEME-AA & $17_{5,13}-17_{4,13}$ & 215452.025 & 87.9 & 8.39 & $\dot{\dagger}$ & & & \\
\hline tEME-EA & $17_{5,13}-17_{4,13}$ & 215452.436 & 87.9 & 5.17 & $\dagger$ & & & \\
\hline tEME-EE' & $17_{5,13}-17_{4,13}$ & 215455.422 & 87.9 & 7.39 & $\ldots$ & $\ldots$ & 0.07 & $\mathrm{CH}_{3} \mathrm{CH}_{2} \mathrm{CN} v_{13} / v_{21}, \mathrm{CH}_{3} \mathrm{CH}_{2} \mathrm{OH}$ \\
\hline tEME-EE & $17_{5,13}-17_{4,13}$ & 215456.151 & 87.9 & 6.90 & 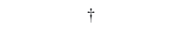 & & & \\
\hline tEME-AE & $17_{5,13}-17_{4,13}$ & 215456.193 & 87.9 & 7.18 & $\dot{\dagger}$ & & & \\
\hline tEME-EA & $17_{5,13}-17_{4,14}$ & 215461.025 & 87.9 & 5.17 & $\ldots$ & $\ldots$ & 0.04 & $\mathrm{CH}_{3} \mathrm{CH}_{2} \mathrm{CN} v_{13} / v_{21}, \mathrm{CH}_{3} \mathrm{CH}_{2} \mathrm{OH}$ \\
\hline tEME-AA & $17_{5,13}-17_{4,14}$ & 215462.304 & 87.9 & 8.39 & $\dot{\dagger}$ & & & \\
\hline tEME-EA & $17_{5,12}-17_{4,14}$ & 215462.994 & 87.9 & 3.22 & $\dagger$ & & & \\
\hline tEME-EE' & $27_{1,27}-26_{0,26}$ & 215478.840 & 144.2 & 21.60 & $\ldots$ & $\ldots$ & 0.24 & $\mathrm{CH}_{3} \mathrm{CH}_{2} \mathrm{CN} v_{13} / v_{21}$ \\
\hline tEME-EE & $27_{1,27}-26_{0,26}$ & 215478.840 & 144.2 & 21.60 & 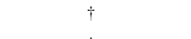 & & & \\
\hline tEME-AE & $27_{1,27}-26_{0,26}$ & 215478.854 & 144.2 & 21.60 & $\dagger$ & & & \\
\hline tEME-EA & $27_{1,27}-26_{0,26}$ & 215479.025 & 144.2 & 21.60 & $\dagger$ & & & \\
\hline tEME-AA & $27_{1,27}-26_{0,26}$ & 215479.039 & 144.2 & 21.60 & $\dagger$ & & & \\
\hline tEME-EE & $16_{5,11}-16_{4,12}$ & 215497.897 & 81.3 & 7.05 & $\ldots$ & $\ldots$ & 0.09 & $\mathrm{CH}_{3} \mathrm{OCH}_{3}, \mathrm{CH}_{3} \mathrm{CH}_{2} \mathrm{CN} v_{13} / v_{21}$ \\
\hline tEME-EA & $16_{5,12}-16_{4,12}$ & 215497.943 & 81.3 & 2.74 & $\stackrel{\dagger}{\dagger}$ & & & \\
\hline tEME-EE' & $16_{5,11}-16_{4,12}$ & 215498.372 & 81.3 & 7.37 & $\dagger$ & & & \\
\hline tEME-AE & $16_{5,11}-16_{4,12}$ & 215498.590 & 81.3 & 7.24 & $\dagger$ & & & \\
\hline tEME-AA & $16_{5,11}-16_{4,12}$ & 215499.524 & 81.3 & 7.82 & $\ldots$ & $\ldots$ & 0.11 & $\mathrm{CH}_{3} \mathrm{OCH}_{3}, \mathrm{CH}_{3} \mathrm{CH}_{2} \mathrm{CN} v_{13} / v_{21}$ \\
\hline tEME-EE' & $16_{5,12}-16_{4,13}$ & 215499.830 & 81.3 & 7.37 & $\dagger$ & & & \\
\hline tEME-EA & $16_{5,11}-16_{4,12}$ & 215499.921 & 81.3 & 5.08 & $\dagger$ & & & \\
\hline tEME-EE & $16_{5,12}-16_{4,13}$ & 215500.305 & 81.3 & 7.05 & $\dagger$ & & & \\
\hline tEME-AE & $16_{5,12}-16_{4,13}$ & 215500.485 & 81.3 & 7.24 & $\dagger$ & & & \\
\hline tEME-EA & $16_{5,11}-16_{4,12}$ & 215504.636 & 81.3 & 5.08 & $\ldots$ & $\ldots$ & 0.05 & $\mathrm{CH}_{3} \mathrm{OCH}_{3}, \mathrm{CH}_{3} \mathrm{CH}_{2} \mathrm{CN} v_{13} / v_{21},{ }^{33} \mathrm{SH}_{2}$ \\
\hline tEME-AA & $16_{5,12}-16_{4,13}$ & 215505.905 & 81.3 & 7.82 & $\dagger$ & & & \\
\hline tEME-EA & $16_{5,12}-16_{4,13}$ & 215506.613 & 81.3 & 2.74 & $\dagger$ & & & \\
\hline tEME-EE & $15_{5,10}-15_{4,11}$ & 215536.404 & 75.1 & 6.94 & 215536.9 & 0.25 & 0.15 & $\mathrm{CH}_{3} \mathrm{OCOH} v_{t}=1$ \\
\hline tEME-EE' & $15_{5,10}-15_{4,11}$ & 215536.720 & 75.1 & 7.08 & $\dot{\dagger}$ & & & \\
\hline tEME-EA & $15_{5,11}-15_{4,11}$ & 215537.002 & 75.1 & 2.05 & $\dagger$ & & & \\
\hline tEME-AE & $15_{5,10}-15_{4,11}$ & 215537.010 & 75.1 & 7.03 & $\dagger$ & & & \\
\hline tEME-EE' & $15_{5,11}-15_{4,12}$ & 215537.169 & 75.1 & 7.08 & $\dagger$ & & & \\
\hline tEME-EE & $15_{5,11}-15_{4,12}$ & 215537.485 & 75.1 & 6.94 & $\dagger$ & & & \\
\hline tEME-AE & $15_{5,11}-15_{4,12}$ & 215537.757 & 75.1 & 7.03 & $\dagger$ & & & \\
\hline tEME-AA & $15_{5,10}-15_{4,11}$ & 215538.650 & 75.1 & 7.25 & $\ldots$ & $\ldots$ & 0.04 & $\mathrm{CH}_{3} \mathrm{OCOH} v_{t}=1, \mathrm{CH}_{3} \mathrm{CH}_{2} \mathrm{CN} v_{13} / v_{21}$ \\
\hline tEME-EA & $15_{5,10}-15_{4,11}$ & 215538.990 & 75.1 & 5.21 & $\dagger$ & & & \\
\hline tEME-AA & $15_{5,10}-15_{4,11}$ & 215538.650 & 75.1 & 7.25 & $\dot{\dagger}$ & & & \\
\hline tEME-EA & $15_{5,10}-15_{4,11}$ & 215538.990 & 75.1 & 5.21 & $\dagger$ & & & \\
\hline tEME-EA & $15_{5,11}-15_{4,12}$ & 215541.270 & 75.1 & 5.21 & $\dagger$ & & & \\
\hline tEME-AA & $15_{5,11}-15_{4,12}$ & 215542.488 & 75.1 & 7.25 & $\dagger$ & & & \\
\hline tEME-EE & $14_{5,9}-14_{4,10}$ & 215567.750 & 69.3 & 6.58 & $\ldots$ & $\ldots$ & 0.17 & $\mathrm{CH}_{3} \mathrm{CH}_{2} \mathrm{CN} v_{20}=1$ \\
\hline tEME-EE' & $14_{5,9}-14_{4,10}$ & 215567.993 & 69.3 & 6.63 & $\dagger$ & & & \\
\hline tEME-EE' & $14_{5,10}-14_{4,11}$ & 215568.043 & 69.3 & 6.63 & $\dot{\dagger}$ & & & \\
\hline tEME-EE & $14_{5,10}-14_{4,11}$ & 215568.286 & 69.3 & 6.58 & $\dot{\dagger}$ & & & \\
\hline tEME-AE & $14_{5,9}-14_{4,10}$ & 215568.316 & 69.3 & 6.61 & $\dagger$ & & & \\
\hline tEME-AE & $14_{5,10}-14_{4,11}$ & 215568.602 & 69.3 & 6.61 & $\dagger$ & & & \\
\hline tEME-EA & $14_{5,10}-14_{4,10}$ & 215568.774 & 69.3 & 1.23 & 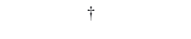 & & & \\
\hline tEME-AA & $14_{5,9}-14_{4,10}$ & 215570.539 & 69.3 & 6.68 & $\ldots$ & $\ldots$ & 0.06 & $\mathrm{CH}_{3} \mathrm{CH}_{2} \mathrm{CN} v_{13} / v_{21}$ \\
\hline tEME-EA & $14_{5,9}-14_{4,10}$ & 215570.772 & 69.3 & 5.45 & $\dagger$ & & & \\
\hline tEME-EA & $14_{5,10}-14_{4,11}$ & 215571.650 & 69.3 & 5.45 & $\dagger$ & & & \\
\hline tEME-AA & $14_{5,10}-14_{4,11}$ & 215572.766 & 69.3 & 6.68 & $\ldots$ & $\ldots$ & 0.04 & $\mathrm{CH}_{3} \mathrm{CH}_{2} \mathrm{CN} v_{13} / v_{21}$ \\
\hline tEME-EA & $14_{5,9}-14_{4,11}$ & 215573.648 & 69.3 & 1.23 & $\dot{\dagger}$ & & & \\
\hline tEME-EE & $13_{5,8}-13_{4,9}$ & 215593.014 & 63.9 & 6.08 & $\ldots$ & $\ldots$ & 0.17 & $\mathrm{CH}_{3} \mathrm{CH}_{2} \mathrm{CN} v_{20}=1, \mathrm{SiO} v=1$ \\
\hline tEME-EE' & $13_{5,9}-13_{4,10}$ & 215593.138 & 63.9 & 6.09 & $\stackrel{\dagger}{\dagger}$ & & & \\
\hline tEME-EE' & $13_{5,8}-13_{4,9}$ & 215593.228 & 63.9 & 6.09 & $\grave{\dagger}$ & & & \\
\hline tEME-EE & $13_{5,9}-13_{4,10}$ & 215593.353 & 63.9 & 6.08 & 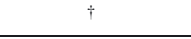 & & & \\
\hline
\end{tabular}


B. Tercero et al.: Trans ethyl methyl ether in space

Table A.2. continued.

\begin{tabular}{|c|c|c|c|c|c|c|c|c|}
\hline Species & $\begin{array}{c}\text { Transition } \\
J_{K_{\mathrm{a}}, K_{\mathrm{c}}}-J_{K_{\mathrm{a}}^{\prime}, K_{\mathrm{c}}^{\prime}}^{\prime}\end{array}$ & $\begin{array}{c}\text { Predicted } \\
\text { frequency }(\mathrm{MHz})\end{array}$ & $\begin{array}{r}E_{\text {upp }} \\
(\mathrm{K})\end{array}$ & $S_{i j}$ & $\begin{array}{c}\text { Observed }^{1} \\
\text { frequency }(\mathrm{MHz})\end{array}$ & $\begin{array}{c}\text { Observed }^{1} \\
T_{\mathrm{MB}}(\mathrm{K})\end{array}$ & $\begin{array}{c}\text { Model }^{2} \\
T_{\mathrm{MB}}(\mathrm{K})\end{array}$ & Blends \\
\hline tEME-AE & $13_{5,8}-13_{4,9}$ & 215593.566 & 63.9 & 6.09 & $\bar{\dagger}$ & & & \\
\hline tEME-AE & $13_{5,9}-13_{4,10}$ & 215593.687 & 63.9 & 6.09 & $\dagger$ & & & \\
\hline tEME-AA & $13_{5,8}-13_{4,9}$ & 215596.207 & 63.9 & 6.11 & $\ldots$ & $\ldots$ & 0.09 & $\mathrm{CH}_{3} \mathrm{CH}_{2}{ }^{13} \mathrm{CN}, \mathrm{SiO} v=1$ \\
\hline tEME-EA & $13_{5,8}-13_{4,9}$ & 215596.289 & 63.9 & 5.58 & $\dagger$ & & & \\
\hline tEME-EA & $13_{5,9}-13_{4,10}$ & 215596.478 & 63.9 & 5.58 & $\dagger$ & & & \\
\hline tEME-EE & $12_{5,7}-12_{4,8}$ & 215613.102 & 58.9 & 5.52 & $\ldots$ & $\ldots$ & 0.18 & $\mathrm{CH}_{3} \mathrm{CH}_{2} \mathrm{OH}, \mathrm{CH}_{3} \mathrm{OCOH} v_{t}=1, \mathrm{CH}_{3} \mathrm{CH}_{2} \mathrm{CN}$ \\
\hline tEME-EE' & $12,{ }_{5,8}-12_{4,9}$ & 215613.172 & 58.9 & 5.53 & $\dagger$ & & & \\
\hline tEME-EE' & $12_{5,7}-12_{4,8}$ & 215613.308 & 58.9 & 5.53 & $\dagger$ & & & \\
\hline tEME-EE & $12_{5,8}-12_{4,9}$ & 215613.378 & 58.9 & 5.52 & $\dagger$ & & & \\
\hline tEME-AE & $12_{5,7}-12_{4,8}$ & 215613.651 & 58.9 & 5.52 & $\dagger$ & & & \\
\hline tEME-AE & $12_{5,8}-12_{4,9}$ & 215613.720 & 58.9 & 5.52 & $\dagger$ & & & \\
\hline tEME-EA & $12_{5,8}-12_{4,9}$ & 215616.405 & 58.9 & 5.37 & $\ldots$ & $\ldots$ & 0.10 & $\mathrm{CH}_{3} \mathrm{CH}_{2} \mathrm{CN}$ \\
\hline tEME-EA & $12_{5,7}-12_{4,8}$ & 215616.490 & 58.9 & 5.37 & $\dagger$ & & & \\
\hline tEME-AA & $12_{5,7}-12_{4,8}$ & 215616.564 & 58.9 & 5.53 & $\dagger$ & & & \\
\hline tEME-AA & $12_{5,8}-12_{4,9}$ & 215617.221 & 58.9 & 5.53 & $\dagger$ & & & \\
\hline tEME-EE & $11_{5,6}-11_{4,7}$ & 215628.800 & 54.3 & 4.94 & $\ldots$ & $\ldots$ & 0.16 & $\mathrm{CH}_{3} \mathrm{CH}_{2} \mathrm{CN}$ \\
\hline tEME-EE' & $11_{5,7}-11_{4,8}$ & 215628.855 & 54.3 & 4.94 & $\doteqdot$ & & & \\
\hline tEME-EE' & $11_{5,6}-11_{4,7}$ & 215629.003 & 54.3 & 4.94 & $\dagger$ & & & \\
\hline tEME-EE & $11_{5,7}-11_{4,8}$ & 215629.058 & 54.3 & 4.94 & $\dagger$ & & & \\
\hline tEME-AE & $11_{5,6}-11_{4,7}$ & 215629.349 & 54.3 & 4.94 & $\dagger$ & & & \\
\hline tEME-AE & $11_{5,7}-11_{4,8}$ & 215629.404 & 54.3 & 4.94 & $\ldots$ & $\ldots$ & 0.10 & $\mathrm{CH}_{3} \mathrm{CH}_{2} \mathrm{CN}$ \\
\hline tEME-EA & $11_{5,7}-11_{4,8}$ & 215632.056 & 54.3 & 4.91 & $\dagger$ & & & \\
\hline tEME-EA & $11_{5,6}-11_{4,7}$ & 215632.229 & 54.3 & 4.91 & $\dagger$ & & & \\
\hline tEME-AA & $11_{5,6}-11_{4,7}$ & 215632.425 & 54.3 & 4.94 & $\dagger$ & & & \\
\hline tEME-AA & $11_{5,7}-11_{4,8}$ & 215632.754 & 54.3 & 4.94 & $\dagger$ & & & \\
\hline tEME-EE & $10_{5,5}-10_{4,6}$ & 215640.803 & 50.0 & 4.35 & 215641.1 & 0.18 & 0.13 & $\mathrm{CH}_{3} \mathrm{COOH}$ \\
\hline tEME-EE' & $10_{5,6}-10_{4,7}$ & 215640.854 & 50.0 & 4.35 & $\dagger$ & & & \\
\hline tEME-EE' & $10_{5,5}-10_{4,6}$ & 215641.007 & 50.0 & 4.35 & $\dagger$ & & & \\
\hline tEME-EE & $10_{5,6}-10_{4,7}$ & 215641.058 & 50.0 & 4.35 & $\dagger$ & & & \\
\hline tEME-AE & $10_{5,5}-10_{4,6}$ & 215641.354 & 50.0 & 4.35 & $\dagger$ & & & \\
\hline tEME-AE & $10_{5,6}-10_{4,7}$ & 215641.405 & 50.0 & 4.35 & $\dagger$ & & & \\
\hline tEME-EA & $10_{5,6}-10_{4,7}$ & 215644.051 & 50.0 & 4.34 & 215644.4 & 0.21 & 0.10 & $\mathrm{CH}_{3} \mathrm{COOH}$ \\
\hline tEME-EA & $10_{5,5}-10_{4,6}$ & 215644.248 & 50.0 & 4.34 & $\dagger$ & & & \\
\hline tEME-AA & $10_{5,5}-10_{4,6}$ & 215644.522 & 50.0 & 4.35 & $\dagger$ & & & \\
\hline tEME-AA & $10_{5,6}-10_{4,7}$ & 215644.676 & 50.0 & 4.35 & $\dagger$ & & & \\
\hline tEME-EE & $9_{5,4}-9_{4,5}$ & 215649.740 & 46.1 & 3.73 & 215650.1 & 0.42 & 0.12 & $\mathrm{CH}_{3} \mathrm{CH}_{2} \mathrm{CN} v_{20}=1$ \\
\hline tEME-EE' & $9_{5,5}-9_{4,6}$ & 215649.791 & 46.1 & 3.73 & 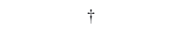 & & & \\
\hline tEME-EE' & $9_{5,4}-9_{4,5}$ & 215649.944 & 46.1 & 3.73 & $\dagger$ & & & \\
\hline tEME-EE & $9_{5,5}-9_{4,6}$ & 215649.995 & 46.1 & 3.73 & $\dagger$ & & & \\
\hline tEME-AE & $9_{5,4}-9_{4,5}$ & 215650.292 & 46.1 & 3.73 & $\dagger$ & & & \\
\hline tEME-AE & $9_{5,5}-9_{4,6}$ & 215650.343 & 46.1 & 3.73 & $\dagger$ & & & \\
\hline tEME-EA & $9_{5,5}-9_{4,6}$ & 215652.990 & 46.1 & 3.73 & 215653.4 & 0.42 & 0.08 & $\mathrm{CH}_{3} \mathrm{CH}_{2} \mathrm{CN} v_{20}=1$ \\
\hline tEME-EA & $9,4-94,5$ & 215653.193 & 46.1 & 3.73 & $\dagger$ & & & \\
\hline tEME-AA & $9_{5,4}-9_{4,5}$ & 215653.509 & 46.1 & 3.73 & $\dagger$ & & & \\
\hline tEME-AA & $9_{5,5}-9_{4,6}$ & 215653.575 & 46.1 & 3.73 & $\dagger$ & & & \\
\hline tEME-EE & $8_{5,3}-8_{4,4}$ & 215656.176 & 42.7 & 3.10 & 215656.4 & 0.43 & 0.11 & $\mathrm{CH}_{3} \mathrm{CH}_{2} \mathrm{CN} v_{20}=1$ \\
\hline tEME-EE' & $8_{5,4}-8_{4,5}$ & 215656.227 & 42.7 & 3.10 & $\dagger$ & & & \\
\hline tEME-EE' & $85,3-84,4$ & 215656.380 & 42.7 & 3.10 & $\dagger$ & & & \\
\hline tEME-EE & $8_{5,4}-8_{4,5}$ & 215656.432 & 42.7 & 3.10 & $\dagger$ & & & \\
\hline tEME-AE & $8,3-84,4$ & 215656.730 & 42.7 & 3.10 & $\dagger$ & & & \\
\hline tEME-AE & $8_{5,4}-8_{4,5}$ & 215656.781 & 42.7 & 3.10 & $\dagger$ & & & \\
\hline tEME-EA & $8,4-8_{4,5}$ & 215659.431 & 42.7 & 3.10 & 215659.8 & 0.36 & 0.09 & $\mathrm{CH}_{3} \mathrm{CH}_{2} \mathrm{CN} v_{20}=1$ \\
\hline tEME-EA & $\begin{array}{l}8_{5,3}-8_{4,4} \\
\text { nats }\end{array}$ & 215659.635 & 42.7 & 3.10 & $\div$ & & & \\
\hline tEME-AA & $8_{5,3}-8_{4,4}$ & 215659.972 & 42.7 & 3.10 & $\dagger$ & & & \\
\hline tEME-AA & $8_{5,4}-8_{4,5}$ & 215659.998 & 42.7 & 3.10 & $\dagger$ & & & \\
\hline tEME-EE & $7_{5,2}-7_{4,3}$ & 215660.620 & 39.6 & 2.43 & 215660.8 & 0.34 & 0.11 & $\mathrm{CH}_{3} \mathrm{CH}_{2} \mathrm{CN} v_{20}=1$ \\
\hline tEME-EE' & $7_{5,3}-7_{4,4}$ & 215660.672 & 39.6 & 2.43 & $\doteqdot$ & & & \\
\hline tEME-EE' & $7_{5,2}-7_{4,3}$ & 215660.825 & 39.6 & 2.43 & $\dagger$ & & & \\
\hline tEME-EE & $7_{5,3}-7_{4,4}$ & 215660.877 & 39.6 & 2.43 & $\dagger$ & & & \\
\hline tEME-AE & $7_{5,2}-7_{4,3}$ & 215661.175 & 39.6 & 2.43 & $\dagger$ & & & \\
\hline tEME-AE & $7_{5,3}-7_{4,4}$ & 215661.227 & 39.6 & 2.43 & $\dagger$ & & & \\
\hline tEME-EE & $6_{5,1}-6_{4,2}$ & 215663.523 & 36.9 & 1.72 & 215663.9 & 0.30 & 0.11 & $\mathrm{CH}_{3} \mathrm{CH}_{2} \mathrm{CN} v_{20}=1$ \\
\hline tEME-EE' & $6,{ }_{5,2}-6_{4,3}$ & 215663.575 & 36.9 & 1.72 & $\dagger$ & & & \\
\hline tEME-EE' & $6_{5,1}-6_{4,2}$ & 215663.728 & 36.9 & 1.72 & $\dagger$ & & & \\
\hline tEME-EE & $6,{ }_{5,2}-6_{4,3}$ & 215663.780 & 36.9 & 1.72 & $\dagger$ & & & \\
\hline tEME-EA & $7_{5,3}-7_{4,4}$ & 215663.879 & 39.6 & 2.43 & $\dagger$ & & & \\
\hline tEME-AE & $6_{5,1}-6_{4,2}$ & 215664.080 & 36.9 & 1.72 & $\dagger$ & & & \\
\hline tEME-EA & $7_{5,2}-7_{4,3}$ & 215664.084 & 39.6 & 2.43 & $\dagger$ & & & \\
\hline tEME-AE & $6_{5,2}-6_{4,3}$ & 215664.131 & 36.9 & 1.72 & $\dagger$ & & & \\
\hline tEME-AA & $7_{5,2}-7_{4,3}$ & 215664.430 & 39.6 & 2.43 & $\dagger$ & & & \\
\hline tEME-AA & $7_{5,3}-7_{4,4}$ & 215664.439 & 39.6 & 2.43 & $\dagger$ & & & \\
\hline tEME-EE & $5_{5,0}-5_{4,1}$ & 215665.279 & 34.6 & 0.92 & $\dagger$ & & & \\
\hline tEME-EE' & $5_{5,1}-5_{4,2}$ & 215665.331 & 34.6 & 0.92 & $\dagger$ & & & \\
\hline
\end{tabular}


A\&A 582, L1 (2015)

Table A.2. continued.

\begin{tabular}{|c|c|c|c|c|c|c|c|c|}
\hline Species & $\begin{array}{c}\text { Transition } \\
J_{K_{\mathrm{a}}, K_{\mathrm{c}}}-J_{K_{\mathrm{a}}^{\prime}, K_{\mathrm{c}}^{\prime}}^{\prime}\end{array}$ & $\begin{array}{c}\text { Predicted } \\
\text { frequency }(\mathrm{MHz})\end{array}$ & $\begin{array}{r}E_{\text {upp }} \\
(\mathrm{K})\end{array}$ & $S_{i j}$ & $\begin{array}{c}\text { Observed }^{1} \\
\text { frequency }(\mathrm{MHz})\end{array}$ & $\begin{array}{c}\text { Observed }^{1} \\
T_{\mathrm{MB}}(\mathrm{K})\end{array}$ & $\begin{array}{c}\text { Model }^{2} \\
T_{\mathrm{MB}}(\mathrm{K})\end{array}$ & Blends \\
\hline tEME-EE' & $5_{5,0}-5_{4,1}$ & 215665.485 & 34.6 & 0.92 & $\bar{\dagger}$ & & & \\
\hline tEME-EE & $5_{5,1}-5_{4,2}$ & 215665.537 & 34.6 & 0.92 & $\dagger$ & & & \\
\hline tEME-AE & $5_{5,0}-5_{4,1}$ & 215665.837 & 34.6 & 0.92 & $\dagger$ & & & \\
\hline tEME-AE & $5_{5,1}-5_{4,2}$ & 215665.889 & 34.6 & 0.92 & $\dagger$ & & & \\
\hline tEME-EA & $6_{5,2}-6_{4,2}$ & 215666.786 & 36.9 & 1.72 & $\ldots$ & $\ldots$ & 0.05 & $\mathrm{CH}_{3} \mathrm{CH}_{2} \mathrm{CN} v=0, v_{20}=1$ \\
\hline tEME-EA & $6_{5,1}-6_{4,2}$ & 215666.991 & 36.9 & 1.72 & $\dagger$ & & & \\
\hline tEME-EA & $5_{5,1}-5_{4,2}$ & 215668.545 & 34.6 & 0.92 & $\dagger$ & & & \\
\hline tEME-EA & $5_{5,0}-5_{4,1}$ & 215668.751 & 34.6 & 0.92 & $\dagger$ & & & \\
\hline tEME-AA & $5_{5,0}-5_{4,1}$ & 215669.103 & 34.6 & 0.92 & $\dagger$ & & & \\
\hline tEME-AA & $5_{5,1}-5_{4,2}$ & 215669.103 & 34.6 & 0.92 & $\dagger$ & & & \\
\hline tEME-EE' & $6_{4,3}-5_{3,3}$ & 216054.535 & 26.5 & 3.73 & 216054.9 & 0.30 & 0.08 & $\mathrm{CH}_{3} \mathrm{CH}_{2} \mathrm{CN} v_{13} / v_{21}$ \\
\hline tEME-EE & $6_{4,3}-5_{3,3}$ & 216054.868 & 26.5 & 3.73 & $\dagger$ & & & \\
\hline tEME-AE & $6_{4,3}-5_{3,3}$ & 216055.095 & 26.5 & 3.73 & $\dagger$ & & & \\
\hline tEME-EE & $6_{4,2}-5_{3,2}$ & 216055.759 & 26.5 & 3.73 & $\dagger$ & & & \\
\hline tEME-EE' & $6_{4,2}-5_{3,2}$ & 216056.093 & 26.5 & 3.73 & 216056.4 & 0.26 & 0.08 & $\mathrm{CH}_{3} \mathrm{CH}_{2} \mathrm{CN} v_{13} / v_{21}$ \\
\hline tEME-AE & $6_{4,2}-5_{3,2}$ & 216056.319 & 26.5 & 3.73 & $\dagger$ & & & \\
\hline tEME-EA & $6_{4,3}-5_{3,3}$ & 216058.222 & 26.5 & 3.69 & 216058.4 & 0.25 & 0.10 & $\mathrm{CH}_{3} \mathrm{CH}_{2} \mathrm{CN} v_{13} / v_{21}$ \\
\hline tEME-EA & $6_{4,2}-5_{3,2}$ & 216058.519 & 26.5 & 3.69 & $\dagger$ & & & \\
\hline tEME-AA & $6_{4,3}-5_{3,2}$ & 216058.597 & 26.5 & 3.73 & $\dagger$ & & & \\
\hline tEME-AA & $6_{4,2}-5_{3,3}$ & 216058.930 & 26.5 & 3.73 & $\dagger$ & & & \\
\hline tEME-EE & $12_{3,9}-11_{2,10}$ & 217007.530 & 40.5 & 4.14 & 217007.7 & 0.13 & 0.08 & $\mathrm{HDCS}, \mathrm{CH}_{3} \mathrm{OCOH}$ \\
\hline tEME-EE' & $12_{3,9}-11_{2,10}$ & 217007.763 & 40.5 & 4.12 & $\dagger$ & & & \\
\hline tEME-AE & $12_{3,9}-11_{2,10}$ & 217007.939 & 40.5 & 4.13 & $\dagger$ & & & \\
\hline tEME-EA & $12_{3,9}-11_{2,10}$ & 217009.762 & 40.5 & 4.16 & 217010.0 & 0.13 & 0.05 & $\mathrm{HDCS}, \mathrm{CH}_{3} \mathrm{OCOH}$ \\
\hline tEME-AA & $12_{3,9}-11_{2,10}$ & 217010.054 & 40.5 & 4.16 & $\dagger$ & & & \\
\hline tEME-AA & $30_{1,29}-29_{2,28}$ & 217164.465 & 182.5 & 13.55 & $\ldots$ & $\ldots$ & 0.07 & $\mathrm{CH}_{3} \mathrm{OCOH}$ \\
\hline tEME-EA & $30_{1,29}-29_{2,28}$ & 217164.508 & 182.5 & 13.55 & $\dagger$ & & & \\
\hline tEME-AE & $30_{1,29}-29_{2,28}$ & 217165.360 & 182.5 & 13.55 & $\dagger$ & & & \\
\hline tEME-EE & $30_{1,29}-29_{2,28}$ & 217165.402 & 182.5 & 13.55 & $\dagger$ & & & \\
\hline tEME-EE' & $30_{1,29}-29_{2,28}$ & 217165.402 & 182.5 & 13.55 & $\dagger$ & & & \\
\hline tEME-AA & $28_{0,28}-27_{1,27}$ & 217940.650 & 154.7 & 22.59 & 217940.7 & 0.44 & 0.25 & $\mathrm{c}-\mathrm{C}_{3} \mathrm{H}_{2}, \mathrm{HCC}^{13} \mathrm{CN} v_{7}=1, \mathrm{CH}_{3} \mathrm{COOCH}_{3}$ \\
\hline tEME-EA & $28_{0,28}-27_{1,27}$ & 217940.651 & 154.7 & 22.59 & 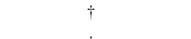 & & & \\
\hline tEME-AE & $28_{0,28}-27_{1,27}$ & 217940.758 & 154.7 & 22.59 & $\dagger$ & & & \\
\hline tEME-EE & $28_{0,28}-27_{1,27}$ & 217940.759 & 154.7 & 22.59 & $\dagger$ & & & \\
\hline tEME-EE' & $28_{0,28}-27_{1,27}$ & 217940.759 & 154.7 & 22.59 & $\dagger$ & & & \\
\hline tEME-EE' & $22_{2,21}-21_{1,20}$ & 219893.140 & 102.2 & 8.31 & $\ldots$ & $\ldots$ & 0.09 & SO \\
\hline tEME-EE & $22_{2,21}-21_{1,20}$ & 219893.140 & 102.2 & 8.31 & $\dagger$ & & & \\
\hline tEME-AE & $22_{2,21}-21_{1,20}$ & 219893.263 & 102.2 & 8.31 & $\dagger$ & & & \\
\hline tEME-EA & $22_{2,21}-21_{1,20}$ & 219894.511 & 102.2 & 8.31 & $\ldots$ & & 0.06 & SO \\
\hline tEME-AA & $22_{2,21}-21_{1,20}$ & 219894.635 & 102.2 & 8.31 & $\dagger$ & & & \\
\hline tEME-EE' & $28_{1,28}-27_{0,27}$ & 222861.487 & 154.8 & 22.63 & 222861.3 & 0.55 & 0.26 & $\mathrm{CH}_{3} \mathrm{OCOH}$ \\
\hline tEME-EE & $28_{1,28}-27_{0,27}$ & 222861.487 & 154.8 & 22.63 & + & & & \\
\hline tEME-AE & $28_{1,28}-27_{0,27}$ & 222861.500 & 154.8 & 22.63 & $\dagger$ & & & \\
\hline tEME-EA & $28_{1,28}-27_{0,27}$ & 222861.655 & 154.8 & 22.63 & $\dagger$ & & & \\
\hline tEME-AA & $28_{1,28}-27_{0,27}$ & 222861.668 & 154.8 & 22.63 & $\dagger$ & & & \\
\hline tEME-EE' & $13_{3,11}-12_{2,10}$ & 222980.574 & 45.5 & 4.38 & 222980.7 & 0.22 & 0.09 & $\mathrm{CH}_{3} \mathrm{O}^{13} \mathrm{COH}$ \\
\hline tEME-EE & $13_{3,11}-12_{2,10}$ & 222980.720 & 45.5 & 4.39 & $\dagger$ & & & \\
\hline tEME-AE & $13_{3,11}-12_{2,10}$ & 222980.951 & 45.5 & 4.39 & $\dagger$ & & & \\
\hline tEME-EA & $13_{3,11}-12_{2,10}$ & 222983.368 & 45.5 & 4.40 & 222983.7 & 0.16 & 0.06 & $\mathrm{CH}_{3} \mathrm{O}^{13} \mathrm{COH}$ \\
\hline tEME-AA & $13_{3,11}-12_{2,10}$ & 222983.672 & 45.5 & 4.40 & $\dagger$ & & & \\
\hline tEME-EE & $16_{2,14}-15_{1,15}$ & 223403.761 & 57.4 & 2.48 & $\ldots$ & $\ldots$ & 0.04 & $\mathrm{CH}_{3} \mathrm{OCH}_{3}, \mathrm{CH}_{3} \mathrm{OCOH} v_{t}=1$ \\
\hline tEME-EE' & $16_{2,14}-15_{1,15}$ & 223403.762 & 57.4 & 2.48 & $\dagger$ & & & \\
\hline tEME-AE & $16_{2,14}-15_{1,15}$ & 223404.001 & 57.4 & 2.48 & $\dagger$ & & & \\
\hline tEME-EA & $16_{2,14}-15_{1,15}$ & 223405.432 & 57.4 & 2.48 & $\ldots$ & $\ldots$ & 0.03 & $\mathrm{CH}_{3} \mathrm{OCH}_{3}$ \\
\hline tEME-AA & $16_{2,14}-15_{1,15}$ & 223405.671 & 57.4 & 2.48 & 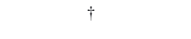 & & & \\
\hline tEME-EE' & $7_{4,4}-6_{3,4}$ & 224103.753 & 29.2 & 3.89 & 224103.98 & 0.63 & 0.10 & $\mathrm{CH}_{3} \mathrm{CH}_{2} \mathrm{CN} v_{13} / v_{21}$ \\
\hline tEME-EE & $7_{4,4}-6_{3,4}$ & 224104.093 & 29.2 & 3.88 & $\doteqdot$ & & & \\
\hline tEME-AE & $7_{4,4}-6_{3,4}$ & 224104.315 & 29.2 & 3.89 & $\dagger$ & & & \\
\hline tEME-EE & $7_{4,3}-6_{3,3}$ & 224104.926 & 29.2 & 3.88 & $\dagger$ & & & \\
\hline tEME-EE' & $7_{4,3}-6_{3,3}$ & 224105.266 & 29.2 & 3.89 & 224105.2 & 0.47 & 0.10 & $\mathrm{CH}_{3} \mathrm{CH}_{2} \mathrm{CN} v_{20}=1$ \\
\hline tEME-AE & $7_{4,3}-6_{3,3}$ & 224105.490 & 29.2 & 3.89 & $\dagger$ & & & \\
\hline tEME-AA & $7_{4,4}-6_{3,3}$ & 224107.456 & 29.2 & 3.90 & 224107.7 & 0.52 & 0.10 & $\mathrm{CH}_{3} \mathrm{CH}_{2} \mathrm{CN} v_{20}=1$ \\
\hline tEME-EA & $74,4-63,4$ & 224107.546 & 29.2 & 3.58 & 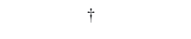 & & & \\
\hline tEME-EA & $7_{4,3}-6_{3,3}$ & 224107.581 & 29.2 & 3.58 & $\dagger$ & & & \\
\hline tEME-AA & $7_{4,3}-6_{3,4}$ & 224108.457 & 29.2 & 3.90 & $\dagger$ & & & \\
\hline tEME-EE & $13_{3,10}-12_{2,11}$ & 225283.674 & 45.5 & 4.33 & $\ldots$ & $\ldots$ & 0.09 & $\mathrm{CH}_{3} \mathrm{CH}_{2} \mathrm{OH}$ \\
\hline tEME-EE' & $13_{3,10}-12_{2,11}$ & 225283.820 & 45.5 & 4.33 & $\dagger$ & & & \\
\hline tEME-AE & $13_{3,10}-12_{2,11}$ & 225284.043 & 45.5 & 4.33 & $\dagger$ & & & \\
\hline tEME-EA & $13_{3,10}-12_{2,11}$ & 225285.990 & 45.5 & 4.34 & 225286.28 & 0.29 & 0.06 & $\mathrm{CH}_{3} \mathrm{CH}_{2} \mathrm{OH}$ \\
\hline tEME-AA & $13_{3,10}-12_{2,11}$ & 225286.286 & 45.5 & 4.34 & $\dagger$ & & & \\
\hline tEME-EE' & $23_{2,22}-22_{1,21}$ & 225494.508 & 111.0 & 8.90 & 225494.4 & 0.12 & 0.10 & \\
\hline tEME-EE & $23_{2,22}-22_{1,21}$ & 225494.508 & 111.0 & 8.90 & $\dagger$ & & & \\
\hline tEME-AE & $23_{2,22}-22_{1,21}$ & 225494.625 & 111.0 & 8.90 & 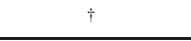 & & & \\
\hline
\end{tabular}


B. Tercero et al.: Trans ethyl methyl ether in space

Table A.2. continued.

\begin{tabular}{|c|c|c|c|c|c|c|c|c|}
\hline Species & $\begin{array}{c}\text { Transition } \\
J_{K_{\mathrm{a}}, K_{\mathrm{c}}}-J_{K_{\mathrm{a}}^{\prime}, K_{\mathrm{c}}^{\prime}}^{\prime}\end{array}$ & $\begin{array}{c}\text { Predicted } \\
\text { frequency }(\mathrm{MHz})\end{array}$ & $\begin{array}{r}E_{\text {upp }} \\
(\mathrm{K})\end{array}$ & $S_{i j}$ & $\begin{array}{c}\text { Observed }^{1} \\
\text { frequency }(\mathrm{MHz})\end{array}$ & $\begin{array}{c}\text { Observed }^{1} \\
T_{\mathrm{MB}}(\mathrm{K})\end{array}$ & $\begin{array}{c}\text { Model }^{2} \\
T_{\mathrm{MB}}(\mathrm{K})\end{array}$ & Blends \\
\hline tEME-EA & $23_{2,22}-22_{1,21}$ & 225495.848 & 111.0 & 8.90 & 225495.9 & 0.12 & 0.07 & \\
\hline tEME-AA & $23_{2,22}-22_{1,21}$ & 225495.965 & 111.0 & 8.90 & $\dagger$ & & & \\
\hline tEME-EA & $29_{0,29}-28_{1,28}$ & 226057.836 & 165.7 & 23.62 & 226057.9 & 0.65 & 0.26 & $\mathrm{CH}_{3} \mathrm{CH}_{2} \mathrm{OH}$ \\
\hline tEME-AA & $29_{0,29}-28_{1,28}$ & 226057.836 & 165.7 & 23.62 & $\dagger$ & & & \\
\hline tEME-EE & $29_{0,29}-28_{1,28}$ & 226057.928 & 165.7 & 23.62 & $\dagger$ & & & \\
\hline tEME-EE' & $29_{0,29}-28_{1,28}$ & 226057.928 & 165.7 & 23.62 & $\dagger$ & & & \\
\hline tEME-AE & $29_{0,29}-28_{1,28}$ & 226057.928 & 165.7 & 23.62 & $\dagger$ & & & \\
\hline tEME-AA & $31_{1,30}-30_{2,29}$ & 227090.552 & 194.5 & 14.51 & $\ldots$ & $\ldots$ & 0.09 & $\mathrm{CH}_{3} \mathrm{OH}$ \\
\hline tEME-EA & $31_{1,30}-30_{2,29}$ & 227090.589 & 194.5 & 14.51 & $\dagger$ & & & \\
\hline tEME-AE & $31_{1,30}-30_{2,29}$ & 227091.390 & 194.5 & 14.51 & $\dagger$ & & & \\
\hline tEME-EE & $31_{1,30}-30_{2,29}$ & 227091.426 & 194.5 & 14.51 & $\dagger$ & & & \\
\hline tEME-EE' & $31_{1,30}-30_{2,29}$ & 227091.426 & 194.5 & 14.51 & $\dagger$ & & & \\
\hline tEME-EE' & $29_{1,29}-28_{0,28}$ & 230291.194 & 165.8 & 23.66 & $\ldots$ & $\ldots$ & 0.27 & $\mathrm{CH}_{3} \mathrm{OH}$ \\
\hline tEME-EE & $29_{1,29}-28_{0,28}$ & 230291.194 & 165.8 & 23.66 & $\dagger$ & & & \\
\hline tEME-AE & $29_{1,29}-28_{0,28}$ & 230291.205 & 165.8 & 23.66 & $\dagger$ & & & \\
\hline tEME-EA & $29_{1,29}-28_{0,28}$ & 230291.345 & 165.8 & 23.66 & $\dagger$ & & & \\
\hline tEME-AA & $29_{1,29}-28_{0,28}$ & 230291.357 & 165.8 & 23.66 & $\dagger$ & & & \\
\hline tEME-EE' & $14_{3,12}-13_{2,11}$ & 230486.881 & 50.9 & 4.59 & $\ldots$ & $\ldots$ & 0.09 & $\mathrm{CO}$ \\
\hline tEME-EE & $14_{3,12}-13_{2,11}$ & 230486.975 & 50.9 & 4.60 & $\dot{\dagger}$ & & & \\
\hline tEME-AE & $14_{3,12}-13_{2,11}$ & 230487.227 & 50.9 & 4.59 & $\dagger$ & & & \\
\hline tEME-EA & $14_{3,12}-13_{2,11}$ & 230489.569 & 50.9 & 4.60 & $\ldots$ & $\ldots$ & 0.07 & $\mathrm{CO}$ \\
\hline tEME-AA & $14_{3,12}-13_{2,11}$ & 230489.869 & 50.9 & 4.60 & $\dagger$ & & & \\
\hline tEME-EE' & $24_{2,23}-23_{1,22}$ & 231063.540 & 120.2 & 9.52 & $\ldots$ & $\ldots$ & 0.11 & OCS \\
\hline tEME-EE & $24_{2,23}-23_{1,22}$ & 231063.540 & 120.2 & 9.52 & $\dagger$ & & & \\
\hline tEME-AE & $24_{2,23}-23_{1,22}$ & 231063.652 & 120.2 & 9.52 & $\dagger$ & & & \\
\hline tEME-EA & $24_{2,23}-23_{1,22}$ & 231064.846 & 120.2 & 9.52 & $\ldots$ & $\ldots$ & 0.08 & OCS \\
\hline tEME-AA & $24_{2,23}-23_{1,22}$ & 231064.958 & 120.2 & 9.52 & $\dagger$ & & & \\
\hline tEME-EE' & $8_{4,5}-7_{3,5}$ & 232151.207 & 32.3 & 4.03 & 232152.1 & 0.47 & 0.11 & ${ }^{13} \mathrm{CH}_{3} \mathrm{CN}, \mathrm{CH}_{3} \mathrm{OCOH} v_{t}=1$ \\
\hline tEME-EE & $8_{4,5}-7_{3,5}$ & 232151.590 & 32.3 & 3.98 & 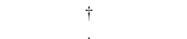 & & & \\
\hline tEME-AE & $8_{4,5}-7_{3,5}$ & 232151.787 & 32.3 & 4.01 & $\dagger$ & & & \\
\hline tEME-EE & $84,4-73,4$ & 232152.098 & 32.3 & 3.98 & $\dagger$ & & & \\
\hline tEME-EE' & $8_{4,4}-7_{3,4}$ & 232152.480 & 32.3 & 4.03 & $\dagger$ & & & \\
\hline tEME-EA & $8_{4,5}-7_{3,4}$ & 232152.521 & 32.3 & 1.00 & $\dagger$ & & & \\
\hline tEME-AE & $8_{4,4}-7_{3,4}$ & 232152.685 & 32.3 & 4.01 & $\dagger$ & & & \\
\hline tEME-AA & $8_{4,5}-7_{3,4}$ & 232154.033 & 32.3 & 4.08 & $\ldots$ & $\ldots$ & 0.06 & ${ }^{13} \mathrm{CH}_{3} \mathrm{CN}, \mathrm{CH}_{3} \mathrm{OCOH} v_{t}=1$ \\
\hline tEME-EA & $8_{4,4}-7_{3,4}$ & 232154.362 & 32.3 & 3.08 & $\dagger$ & & & \\
\hline tEME-EA & $8_{4,5}-7_{3,5}$ & 232155.426 & 32.3 & 3.08 & $\dagger$ & & & \\
\hline tEME-AA & $8_{4,4}-73,5$ & 232156.540 & 32.3 & 4.08 & $\ldots$ & $\ldots$ & 0.04 & ${ }^{13} \mathrm{CH}_{3} \mathrm{CN}, \mathrm{CH}_{3} \mathrm{OCOH} v_{t}=1$ \\
\hline tEME-EA & $8_{4,4}-7_{3,5}$ & 232157.268 & 32.3 & 1.00 & $\dagger$ & & & \\
\hline tEME-EE & $14_{3,11}-13_{2,12}$ & 233622.462 & 51.0 & 4.51 & $\ldots$ & $\ldots$ & 0.10 & $\mathrm{CH}_{3} \mathrm{OCOH} v_{t}=0,1$ \\
\hline tEME-EE' & $14_{3,11}-13_{2,12}$ & 233622.556 & 51.0 & 4.51 & $\dagger$ & & & \\
\hline tEME-AE & $14_{3,11}-13_{2,12}$ & 233622.806 & 51.0 & 4.51 & $\dagger$ & & & \\
\hline tEME-EA & $14_{3,11}-13_{2,12}$ & 233624.824 & 51.0 & 4.52 & $\ldots$ & $\ldots$ & 0.06 & $\mathrm{CH}_{3} \mathrm{OCOH} v_{t}=0,1$ \\
\hline tEME-AA & $14_{3,11}-13_{2,12}$ & 233625.121 & 51.0 & 4.52 & $\dagger$ & & & \\
\hline tEME-EA & $30_{0,30}-29_{1,29}$ & 234130.523 & 177.0 & 24.66 & $\ldots$ & $\ldots$ & 0.27 & $\mathrm{CH}_{3} \mathrm{CH}_{2} \mathrm{CN} v_{13} / v_{21}$ \\
\hline tEME-AA & $30_{0,30}-29_{1,29}$ & 234130.524 & 177.0 & 24.66 & $\dagger$ & & & \\
\hline tEME-EE & $30_{0,30}-29_{1,29}$ & 234130.601 & 177.0 & 24.66 & $\dagger$ & & & \\
\hline tEME-EE' & $30_{0,30}-29_{1,29}$ & 234130.601 & 177.0 & 24.66 & $\dagger$ & & & \\
\hline tEME-AE & $30_{0,30}-29_{1,29}$ & 234130.602 & 177.0 & 24.66 & $\dagger$ & & & \\
\hline tEME-EE & $17_{2,15}-16_{1,16}$ & 235247.484 & 64.0 & 2.37 & 235247.6 & 0.09 & 0.05 & \\
\hline tEME-EE' & $17_{2,15}-16_{1,16}$ & 235247.485 & 64.0 & 2.37 & $\dagger$ & & & \\
\hline tEME-AE & $17_{2,15}-16_{1,16}$ & 235247.731 & 64.0 & 2.37 & $\doteqdot$ & & & \\
\hline tEME-EA & $17_{2,15}-16_{1,16}$ & 235249.156 & 64.0 & 2.37 & 235249.6 & 0.14 & 0.03 & U-line \\
\hline tEME-AA & $17_{2,15}-16_{1,16}$ & 235249.403 & 64.0 & 2.37 & $\doteqdot$ & & & \\
\hline tEME-EE' & $25_{2,24}-24_{1,23}$ & 236614.358 & 129.8 & 10.19 & 236614.4 & 0.30 & 0.11 & $\mathrm{CH}_{3} \mathrm{COOCH}_{3}$ \\
\hline tEME-EE & $25_{2,24}-24_{1,23}$ & 236614.358 & 129.8 & 10.19 & $\dagger$ & & & \\
\hline tEME-AE & $25_{2,24}-24_{1,23}$ & 236614.464 & 129.8 & 10.19 & $\dagger$ & & & \\
\hline tEME-EA & $25_{2,24}-24_{1,23}$ & 236615.628 & 129.8 & 10.19 & $\dagger$ & & 0.08 & $\mathrm{CH}_{3} \mathrm{COOCH}_{3}$ \\
\hline tEME-AA & $25_{2,24}-24_{1,23}$ & 236615.733 & 129.8 & 10.19 & $\dagger$ & & & \\
\hline tEME-AA & $32_{1,31}-31_{2,30}$ & 236906.877 & 206.9 & 15.50 & 236907.2 & 0.30 & 0.10 & $\mathrm{CH}_{3} \mathrm{SH}, \mathrm{HC}_{3} \mathrm{~N} v_{6}=1$ \\
\hline tEME-EA & $32_{1,31}-31_{2,30}$ & 236906.908 & 206.9 & 15.50 & 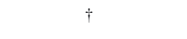 & & & \\
\hline tEME-AE & $32_{1,31}-31_{2,30}$ & 236907.656 & 206.9 & 15.50 & $\dagger$ & & & \\
\hline tEME-EE & $32_{1,31}-31_{2,30}$ & 236907.687 & 206.9 & 15.50 & $\dagger$ & & & \\
\hline tEME-EE' & $32_{1,31}-31_{2,30}$ & 236907.687 & 206.9 & 15.50 & $\dagger$ & & & \\
\hline tEME-EE' & $30_{1,30}-29_{0,29}$ & 237763.517 & 177.1 & 24.69 & 237763.6 & 0.48 & 0.28 & $\mathrm{CH}_{2} \mathrm{CHCN}$ \\
\hline tEME-EE & $30_{1,30}-29_{0,29}$ & 237763.517 & 177.1 & 24.69 & $\dagger$ & & & \\
\hline tEME-AE & $30_{1,30}-29_{0,29}$ & 237763.527 & 177.1 & 24.69 & $\dagger$ & & & \\
\hline tEME-EA & $30_{1,30}-29_{0,29}$ & 237763.653 & 177.1 & 24.69 & $\dagger$ & & & \\
\hline tEME-AA & $30_{1,30}-29_{0,29}$ & 237763.664 & 177.1 & 24.69 & $\dagger$ & & & \\
\hline tEME-EE' & $15_{3,13}-14_{2,12}$ & 237865.715 & 56.7 & 4.79 & $\ldots$ & $\ldots$ & 0.10 & $\mathrm{CH}_{2} \mathrm{CHCN}$ \\
\hline tEME-EE & $15_{3,13}-14_{2,12}$ & 237865.778 & 56.7 & 4.80 & $\dagger$ & & & \\
\hline tEME-AE & $15_{3,13}-14_{2,12}$ & 237866.042 & 56.7 & 4.80 & $\dagger$ & & & \\
\hline tEME-EA & $15_{3,13}-14_{2,12}$ & 237868.339 & 56.7 & 4.80 & $\ldots$ & $\ldots$ & 0.08 & $\mathrm{CH}_{2} \mathrm{CHCN}$ \\
\hline
\end{tabular}


A\&A 582, L1 (2015)

Table A.2. continued.

\begin{tabular}{|c|c|c|c|c|c|c|c|c|}
\hline Species & $\begin{array}{c}\text { Transition } \\
J_{K_{\mathrm{a}}, K_{\mathrm{c}}}-J_{K_{\mathrm{a}}^{\prime}, K_{\mathrm{c}}^{\prime}}^{\prime}\end{array}$ & $\begin{array}{c}\text { Predicted } \\
\text { frequency }(\mathrm{MHz})\end{array}$ & $\begin{array}{r}E_{\text {upp }} \\
(\mathrm{K})\end{array}$ & $S_{i j}$ & $\begin{array}{c}\text { Observed }^{1} \\
\text { frequency }(\mathrm{MHz})\end{array}$ & $\begin{array}{c}\text { Observed }^{1} \\
T_{\mathrm{MB}}(\mathrm{K})\end{array}$ & $\begin{array}{c}\text { Model }^{2} \\
T_{\mathrm{MB}}(\mathrm{K})\end{array}$ & Blends \\
\hline tEME-AA & $15_{3,13}-14_{2,12}$ & 237868.635 & 56.7 & 4.80 & $\bar{\dagger}$ & & & \\
\hline tEME-EA & $9_{4,6}-8_{3,5}$ & 240195.817 & 35.8 & 1.64 & $\ldots$ & $\ldots$ & 0.10 & $\mathrm{CH}_{2} \mathrm{CHCN} v_{15}=1$ \\
\hline tEME-EE & $9_{4,5}-8,5$ & 240196.101 & 35.8 & 3.88 & $\dagger$ & & & \\
\hline tEME-EE' & $94,6-83,6$ & 240196.396 & 35.8 & 3.88 & $\dagger$ & & & \\
\hline tEME-EE' & $9_{4,5}-8_{3,5}$ & 240196.642 & 35.8 & 3.88 & $\dagger$ & & & \\
\hline tEME-AE & $9_{4,5}-8_{3,5}$ & 240196.777 & 35.8 & 3.88 & $\dagger$ & & & \\
\hline tEME-EE & $9_{4,6}-8_{3,6}$ & 240196.937 & 35.8 & 3.88 & $\dagger$ & & & \\
\hline tEME-AE & $9_{4,6}-8_{3,6}$ & 240197.045 & 35.8 & 3.88 & $\dagger$ & & & \\
\hline tEME-AA & $9_{4,6}-8_{3,5}$ & 240197.196 & 35.8 & 3.88 & $\dagger$ & & & \\
\hline tEME-EA & $94,5-83,5$ & 240197.654 & 35.8 & 3.88 & 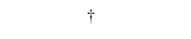 & & & \\
\hline tEME-EA & $9_{4,6}-8_{3,6}$ & 240201.478 & 35.8 & 2.63 & $\ldots$ & $\ldots$ & 0.05 & $\mathrm{CH}_{2} \mathrm{CHCN} v_{15}=1$ \\
\hline tEME-AA & $94,5-83,6$ & 240202.719 & 35.8 & 4.28 & $\dagger$ & & & \\
\hline tEME-EA & $9_{4,5}-8_{3,6}$ & 240203.315 & 35.8 & 1.64 & 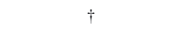 & & & \\
\hline tEME-EE & $15_{3,12}-14_{2,13}$ & 242035.318 & 56.8 & 4.68 & $\ldots$ & $\ldots$ & 0.10 & $\mathrm{CH}_{2} \mathrm{DCN}$ \\
\hline tEME-EE' & $15_{3,12}-14_{2,13}$ & 242035.380 & 56.8 & 4.68 & $\dagger$ & & & \\
\hline tEME-AE & $15_{3,12}-14_{2,13}$ & 242035.647 & 56.8 & 4.68 & $\dagger$ & & & \\
\hline tEME-EA & $15_{3,12}-14_{2,13}$ & 242037.704 & 56.8 & 4.68 & $\ldots$ & $\ldots$ & 0.07 & $\mathrm{CH}_{2} \mathrm{DCN}$ \\
\hline tEME-EA & $15_{3,12}-14_{2,13}$ & 242038.002 & 56.8 & 4.68 & $\dagger$ & & & \\
\hline tEME-EE' & $26_{2,25}-25_{1,24}$ & 242161.785 & 139.8 & 10.89 & $\ldots$ & $\ldots$ & 0.32 & $\mathrm{CH}_{3} \mathrm{CH}_{2} \mathrm{CN} v_{20}=1$ \\
\hline tEME-EE & $26_{2,25}-25_{1,24}$ & 242161.785 & 139.8 & 10.89 & $\dagger$ & & & \\
\hline tEME-AE & $26_{2,25}-25_{1,24}$ & 242161.884 & 139.8 & 10.89 & $\dagger$ & & & \\
\hline tEME-EA & $26_{2,25}-25_{1,24}$ & 242163.015 & 139.8 & 10.89 & $\dagger$ & & & \\
\hline tEME-AA & $26_{2,25}-25_{1,24}$ & 242163.114 & 139.8 & 10.89 & $\doteqdot$ & & & \\
\hline tEME-EA & $31_{0,31}-30_{1,30}$ & 242163.369 & 188.7 & 25.69 & $\dagger$ & & & \\
\hline tEME-AA & $31_{0,31}-30_{1,30}$ & 242163.371 & 188.7 & 25.69 & $\dagger$ & & & \\
\hline tEME-EE & $31_{0,31}-30_{1,30}$ & 242163.434 & 188.7 & 25.69 & $\dagger$ & & & \\
\hline tEME-EE' & $31_{0,31}-30_{1,30}$ & 242163.434 & 188.7 & 25.69 & $\dagger$ & & & \\
\hline tEME-AE & $31_{0,31}-30_{1,30}$ & 242163.436 & 188.7 & 25.69 & $\dagger$ & & & \\
\hline tEME-EE' & $16_{3,14}-15_{2,13}$ & 245103.550 & 62.9 & 4.99 & $\ldots$ & $\ldots$ & 0.10 & $\mathrm{CH}_{3} \mathrm{CH}_{2}{ }^{13} \mathrm{CN}$ \\
\hline tEME-EE & $16_{3,14}-15_{2,13}$ & 245103.592 & 62.9 & 4.99 & $\dagger$ & & & \\
\hline tEME-AE & $16_{3,14}-15_{2,13}$ & 245103.864 & 62.9 & 4.99 & $\dagger$ & & & \\
\hline tEME-EA & $16_{3,14}-15_{2,13}$ & 245106.133 & 62.9 & 4.99 & 245106.4 & 0.32 & 0.07 & $\mathrm{CH}_{2} \mathrm{OHCHO}$ \\
\hline tEME-AA & $16_{3,14}-15_{2,13}$ & 245106.426 & 62.9 & 5.00 & $\dagger$ & & & \\
\hline tEME-EE' & $31_{1,31}-30_{0,30}$ & 245274.088 & 188.8 & 25.71 & 245274.3 & 0.40 & 0.29 & ${ }^{34} \mathrm{SO}_{2}$ \\
\hline tEME-EE & $31_{1,31}-30_{0,30}$ & 245274.088 & 188.8 & 25.71 & $\dagger$ & & & \\
\hline tEME-AE & $31_{1,31}-30_{0,30}$ & 245274.098 & 188.8 & 25.71 & $\dagger$ & & & \\
\hline tEME-EA & $31_{1,31}-30_{0,30}$ & 245274.211 & 188.8 & 25.71 & $\dagger$ & & & \\
\hline tEME-AA & $31_{1,31}-30_{0,30}$ & 245274.221 & 188.8 & 25.71 & $\dagger$ & & & \\
\hline tEME-AA & $33_{1,32}-32_{2,31}$ & 246605.346 & 219.6 & 16.51 & 246606.0 & 0.36 & 0.11 & $\mathrm{CH}_{3} \mathrm{OCH}_{3}$ \\
\hline tEME-EA & $33_{1,32}-32_{2,31}$ & 246605.372 & 219.6 & 16.51 & $\dagger$ & & & \\
\hline tEME-AE & $33_{1,32}-32_{2,31}$ & 246606.066 & 219.6 & 16.51 & $\dagger$ & & & \\
\hline tEME-EE & $33_{1,32}-32_{2,31}$ & 246606.092 & 219.6 & 16.51 & $\dagger$ & & & \\
\hline tEME-EE' & $33_{1,32}-32_{2,31}$ & 246606.092 & 219.6 & 16.51 & $\dagger$ & & & \\
\hline tEME-EE & $18_{2,16}-17_{1,17}$ & 247478.246 & 71.1 & 2.26 & 247478.0 & 0.16 & 0.05 & $\mathrm{CH}_{3} \mathrm{OCOH} v_{t}=1$ \\
\hline tEME-EE' & $18_{2,16}-17_{1,17}$ & 247478.247 & 71.1 & 2.26 & $\dagger$ & & & \\
\hline tEME-AE & $18_{2,16}-17_{1,17}$ & 247478.501 & 71.1 & 2.26 & $\dagger$ & & & \\
\hline tEME-EA & $18_{2,16}-17_{1,17}$ & 247479.920 & 71.1 & 2.26 & 247480.0 & 0.16 & 0.03 & $\mathrm{CH}_{3} \mathrm{OCOH} v_{t}=1$ \\
\hline tEME-AA & $18_{2,16}-17_{1,17}$ & 247480.175 & 71.1 & 2.26 & $\dagger$ & & & \\
\hline tEME-EE' & $27_{2,26}-26_{1,25}$ & 247721.097 & 150.2 & 11.63 & 247721.2 & 0.23 & 0.11 & \\
\hline tEME-EE & $27_{2,26}-26_{1,25}$ & 247721.097 & 150.2 & 11.63 & $\dot{\dagger}$ & & & \\
\hline tEME-AE & $27_{2,26}-26_{1,25}$ & 247721.190 & 150.2 & 11.63 & $\dagger$ & & & \\
\hline tEME-EA & $27_{2,26}-26_{1,25}$ & 247722.285 & 150.2 & 11.63 & $\dagger$ & & & \\
\hline tEME-AA & $27_{2,26}-26_{1,25}$ & 247722.378 & 150.2 & 11.63 & $\dagger$ & & & \\
\hline tEME-EA & $10_{4,7}-9_{3,6}$ & 248234.223 & 39.7 & 2.12 & 248235.7 & 0.31 & 0.15 & U-line \\
\hline tEME-EE & $10_{4,6}-9_{3,6}$ & 248235.232 & 39.7 & 3.50 & $\dagger$ & & & \\
\hline tEME-AA & $10_{4,7}-9,6$ & 248235.506 & 39.7 & 4.48 & $\dagger$ & & & \\
\hline tEME-EA & $10_{4,6}-9,6$ & 248236.059 & 39.7 & 2.36 & $\dagger$ & & & \\
\hline tEME-AE & $10_{4,6}-9_{3,6}$ & 248236.076 & 39.7 & 3.66 & $\dagger$ & & & \\
\hline tEME-EE' & $10_{4,6}-9,6$ & 248236.093 & 39.7 & 3.79 & $\dagger$ & & & \\
\hline tEME-EE' & $10_{4,7}-9_{3,7}$ & 248239.115 & 39.7 & 3.79 & 248239.8 & 0.07 & 0.09 & U-line \\
\hline tEME-AE & $10_{4,7}-9_{3,7}$ & 248239.913 & 39.7 & 3.66 & $\dagger$ & & & \\
\hline tEME-EE & $10_{4,7}-9_{3,7}$ & 248239.977 & 39.7 & 3.50 & $\dagger$ & & & \\
\hline tEME-EA & $10_{4,7}-9_{3,7}$ & 248245.234 & 39.7 & 2.36 & $\ldots$ & $\ldots$ & 0.06 & $\mathrm{CH}_{3} \mathrm{COCH}_{3}$ \\
\hline tEME-AA & $10_{4,6}-9_{3,7}$ & 248246.569 & 39.7 & 4.48 & $\dddot{\dagger}$ & & & \\
\hline tEME-EA & $10_{4,6}-9_{3,7}$ & 248247.070 & 39.7 & 2.12 & $\grave{\dagger}$ & & & \\
\hline tEME-EE & $10_{4,6}-9,7$ & 248248.602 & 39.7 & 0.98 & 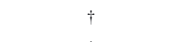 & & & \\
\hline tEME-AE & $10_{4,6}-9_{3,7}$ & 248250.368 & 39.7 & 0.82 & $\dagger$ & & & \\
\hline tEME-EE' & $10_{4,6}-9_{3,7}$ & 248251.399 & 39.7 & 0.69 & $\dagger$ & & & \\
\hline tEME-EA & $32_{0,32}-31_{1,31}$ & 250160.753 & 200.8 & 26.72 & $\ldots$ & $\ldots$ & 0.29 & ${ }^{34} \mathrm{SO}_{2}$ \\
\hline tEME-AA & $32_{0,32}-31_{1,31}$ & 250160.756 & 200.8 & 26.72 & $\dagger$ & & & \\
\hline tEME-EE & $32_{0,32}-31_{1,31}$ & 250160.807 & 200.8 & 26.72 & $\dagger$ & & & \\
\hline tEME-EE' & $32_{0,32}-31_{1,31}$ & 250160.807 & 200.8 & 26.72 & $\dagger$ & & & \\
\hline tEME-AE & $32_{0,32}-31_{1,31}$ & 250160.810 & 200.8 & 26.72 & $\dagger$ & & & \\
\hline
\end{tabular}


B. Tercero et al.: Trans ethyl methyl ether in space

Table A.2. continued.

\begin{tabular}{|c|c|c|c|c|c|c|c|c|}
\hline Species & $\begin{array}{c}\text { Transition } \\
J_{K_{\mathrm{a}}, K_{\mathrm{c}}}-J_{K_{\mathrm{a}}^{\prime}, K_{\mathrm{c}}^{\prime}}^{\prime}\end{array}$ & $\begin{array}{c}\text { Predicted } \\
\text { frequency }(\mathrm{MHz})\end{array}$ & $\begin{array}{r}E_{\text {upp }} \\
(\mathrm{K})\end{array}$ & $S_{i j}$ & $\begin{array}{c}\text { Observed }^{1} \\
\text { frequency }(\mathrm{MHz})\end{array}$ & $\begin{array}{c}\text { Observed }^{1} \\
T_{\mathrm{MB}}(\mathrm{K})\end{array}$ & $\begin{array}{c}\text { Model }^{2} \\
T_{\mathrm{MB}}(\mathrm{K})\end{array}$ & Blends \\
\hline tEME-EE & $16_{3,13}-15_{2,14}$ & 250534.903 & 62.9 & 4.83 & 250535.0 & 0.16 & 0.11 & \\
\hline tEME-EE' & $16_{3,13}-15_{2,14}$ & 250534.946 & 62.9 & 4.83 & $\dagger$ & & & \\
\hline tEME-AE & $16_{3,13}-15_{2,14}$ & 250535.223 & 62.9 & 4.83 & $\dagger$ & & & \\
\hline tEME-EA & $16_{3,13}-15_{2,14}$ & 250537.299 & 62.9 & 4.83 & 250537.5 & 0.11 & 0.08 & \\
\hline tEME-AA & $16_{3,13}-15_{2,14}$ & 250537.598 & 62.9 & 4.83 & $\dagger$ & & & \\
\hline tEME-EE' & $17_{3,15}-16_{2,14}$ & 252188.288 & 69.5 & 5.19 & 252188.5 & 0.59 & 0.11 & $\mathrm{CH}_{3} \mathrm{CH}_{2} \mathrm{CN} v_{13} / v_{21}$ \\
\hline tEME-EE & $17_{3,15}-16_{2,14}$ & 252188.318 & 69.5 & 5.19 & $\dagger$ & & & \\
\hline tEME-AE & $17_{3,15}-16_{2,14}$ & 252188.592 & 69.5 & 5.19 & $\dagger$ & & & \\
\hline tEME-EA & $17_{3,15}-16_{2,14}$ & 252190.846 & 69.5 & 5.19 & $\ldots$ & $\ldots$ & 0.08 & $\mathrm{CH}_{3} \mathrm{CH}_{2} \mathrm{CN} v_{13} / v_{21}$ \\
\hline tEME-AA & $17_{3,15}-16_{2,14}$ & 252191.135 & 69.5 & 5.19 & $\dagger$ & & & \\
\hline tEME-EE' & $32_{1,32}-31_{0,31}$ & 252818.715 & 200.8 & 26.74 & $\ldots$ & $\ldots$ & 0.29 & $\mathrm{CH}_{3} \mathrm{OH}$ \\
\hline tEME-EE & $32_{1,32}-31_{0,31}$ & 252818.715 & 200.8 & 26.74 & $\dagger$ & & & \\
\hline tEME-AE & $32_{1,32}-31_{0,31}$ & 252818.724 & 200.8 & 26.74 & $\dagger$ & & & \\
\hline tEME-EA & $32_{1,32}-31_{0,31}$ & 252818.826 & 200.8 & 26.74 & $\dagger$ & & & \\
\hline tEME-AA & $32_{1,32}-31_{0,31}$ & 252818.835 & 200.8 & 26.74 & $\dagger$ & & & \\
\hline tEME-EE' & $28_{2,27}-27_{1,26}$ & 253307.713 & 161.0 & 12.42 & $\ldots$ & $\ldots$ & 0.12 & ${ }^{13} \mathrm{CH}_{3} \mathrm{OH}$ \\
\hline tEME-EE & $28_{2,27}-27_{1,26}$ & 253307.713 & 161.0 & 12.42 & $\dot{\dagger}$ & & & \\
\hline tEME-AE & $28_{2,27}-27_{1,26}$ & 253307.800 & 161.0 & 12.42 & $\dagger$ & & & \\
\hline tEME-EA & $28_{2,27}-27_{1,26}$ & 253308.856 & 161.0 & 12.42 & $\dagger$ & & & \\
\hline tEME-AA & $28_{2,27}-27_{1,26}$ & 253308.943 & 161.0 & 12.42 & $\dagger$ & & & \\
\hline tEME-EE & $5_{5,0}-4_{4,0}$ & 255923.090 & 34.6 & 4.50 & 255923.1 & 0.42 & 0.29 & $\mathrm{CH}_{3} \mathrm{CH}_{2} \mathrm{CN}$ \\
\hline tEME-EE' & $5_{5,1}-4_{4,1}$ & 255923.151 & 34.6 & 4.50 & $\dagger$ & & & \\
\hline tEME-EE' & $5_{5,0}-4_{4,0}$ & 255923.293 & 34.6 & 4.50 & $\dagger$ & & & \\
\hline tEME-EE & $5_{5,1}-4_{4,1}$ & 255923.354 & 34.6 & 4.50 & $\dagger$ & & & \\
\hline tEME-AE & $5_{5,0}-4_{4,0}$ & 255923.648 & 34.6 & 4.50 & $\dagger$ & & & \\
\hline tEME-AE & $5_{5,1}-4_{4,1}$ & 255923.709 & 34.6 & 4.50 & $\dagger$ & & & \\
\hline tEME-EA & $5_{5,1}-4_{4,1}$ & 255926.362 & 34.6 & 4.50 & 255926.5 & 0.37 & 0.20 & $\mathrm{SO}_{2}$ \\
\hline tEME-EA & $5_{5,0}-4_{4,0}$ & 255926.565 & 34.6 & 4.50 & $\dagger$ & & & \\
\hline tEME-AA & $5_{5,1}-4_{4,0}$ & 255926.920 & 34.6 & 4.50 & $\dagger$ & & & \\
\hline tEME-AA & $5_{5,0}-4_{4,1}$ & 255926.920 & 34.6 & 4.50 & $\dagger$ & & & \\
\hline tEME-AA & $34_{1,33}-33_{2,32}$ & 256180.110 & 232.7 & 17.54 & 256180.5 & 0.28 & 0.11 & $\mathrm{CH}_{3} \mathrm{OCOH} v_{t}=1$ \\
\hline tEME-EA & $34_{1,33}-33_{2,32}$ & 256180.131 & 232.7 & 17.54 & 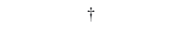 & & & \\
\hline tEME-AE & $34_{1,33}-33_{2,32}$ & 256180.771 & 232.7 & 17.54 & $\dagger$ & & & \\
\hline tEME-EE & $34_{1,33}-33_{2,32}$ & 256180.792 & 232.7 & 17.54 & $\dagger$ & & & \\
\hline tEME-EE' & $34_{1,33}-33_{2,32}$ & 256180.792 & 232.7 & 17.54 & $\dagger$ & & & \\
\hline tEME-EA & $11_{4,8}-10_{3,7}$ & 256265.991 & 43.9 & 2.59 & $\ldots$ & $\ldots$ & 0.10 & $\mathrm{SO}_{2}$ \\
\hline tEME-AA & $11_{4,8}-10_{3,7}$ & 256267.169 & 43.9 & 4.68 & $\dagger$ & & & \\
\hline tEME-EE & $11_{4,7}-10_{3,7}$ & 256267.504 & 43.9 & 3.09 & $\dagger$ & & & \\
\hline tEME-EA & $11_{4,7}-10_{3,7}$ & 256267.843 & 43.9 & 2.09 & $\dagger$ & & & \\
\hline tEME-AE & $11_{4,8}-10_{3,8}$ & 256280.321 & 43.9 & 3.24 & $\ldots$ & $\ldots$ & 0.07 & $\mathrm{CH}_{3} \mathrm{OCOH} v_{t}=1$ \\
\hline tEME-EE & $11_{4,8}-10_{3,8}$ & 256280.554 & 43.9 & 3.09 & $\dagger$ & & & \\
\hline tEME-AE & $11_{4,7}-10_{3,8}$ & 256290.759 & 43.9 & 1.44 & $\ldots$ & $\ldots$ & 0.07 & $\mathrm{CH}_{3} \mathrm{CCH}$ \\
\hline tEME-EE' & $11_{4,7}-10_{3,8}$ & 256291.611 & 43.9 & 1.30 & $\dagger$ & & & \\
\hline tEME-EA & $33_{0,33}-32_{1,32}$ & 258126.741 & 213.2 & 27.74 & $\ldots$ & $\ldots$ & 0.29 & $\mathrm{CH}_{3} \mathrm{CN} v_{8}=1, \mathrm{CH}_{3} \mathrm{OCOH}$ \\
\hline tEME-AA & $33_{0,33}-32_{1,32}$ & 258126.744 & 213.2 & 27.74 & $\dagger$ & & & \\
\hline tEME-EE & $33_{0,33}-32_{1,32}$ & 258126.784 & 213.2 & 27.74 & $\dagger$ & & & \\
\hline tEME-EE' & $33_{0,33}-32_{1,32}$ & 258126.784 & 213.2 & 27.74 & $\dagger$ & & & \\
\hline tEME-AE & $33_{0,33}-32_{1,32}$ & 258126.787 & 213.2 & 27.74 & $\dagger$ & & & \\
\hline tEME-EE' & $29_{2,28}-28_{1,27}$ & 258936.835 & 172.1 & 13.24 & $\ldots$ & $\ldots$ & 0.12 & $\mathrm{SO}_{2}$ \\
\hline tEME-EE & $29_{2,28}-28_{1,27}$ & 258936.835 & 172.1 & 13.24 & $\dagger$ & & & \\
\hline tEME-AE & $29_{2,28}-28_{1,27}$ & 258936.915 & 172.1 & 13.24 & $\dagger$ & & & \\
\hline tEME-EA & $29_{2,28}-28_{1,27}$ & 258937.931 & 172.1 & 13.24 & $\dagger$ & & & \\
\hline tEME-AA & $29_{2,28}-28_{1,27}$ & 258938.012 & 172.1 & 13.24 & $\dagger$ & & & \\
\hline tEME-EE' & $18_{3,16}-17_{2,15}$ & 259109.696 & 76.5 & 5.39 & $\ldots$ & $\ldots$ & 0.11 & $\mathrm{CH}_{3} \mathrm{OCOH}$ \\
\hline tEME-EE & $18_{3,16}-17_{2,15}$ & 259109.717 & 76.5 & 5.39 & $\dagger$ & & & \\
\hline tEME-AE & $18_{3,16}-17_{2,15}$ & 259109.992 & 76.5 & 5.39 & $\dagger$ & & & \\
\hline tEME-EA & $18_{3,16}-17_{2,15}$ & 259112.236 & 76.5 & 5.39 & $\ldots$ & $\ldots$ & 0.08 & $\mathrm{CH}_{3} \mathrm{OCOH}$ \\
\hline tEME-AA & $18_{3,16}-17_{2,15}$ & 259112.522 & 76.5 & 5.39 & $\dagger$ & & & \\
\hline tEME-EE & $17_{3,14}-16_{2,15}$ & 259135.164 & 69.5 & 4.97 & $\ldots$ & $\ldots$ & 0.11 & $\mathrm{CH}_{3} \mathrm{OCOH}$ \\
\hline tEME-EE' & $17_{3,14}-16_{2,15}$ & 259135.193 & 69.5 & 4.97 & $\dagger$ & & & \\
\hline tEME-AE & $17_{3,14}-16_{2,15}$ & 259135.477 & 69.5 & 4.97 & $\dagger$ & & & \\
\hline tEME-EA & $17_{3,14}-16_{2,15}$ & 259137.561 & 69.5 & 4.97 & $\ldots$ & $\ldots$ & 0.08 & $\mathrm{CH}_{3} \mathrm{OCOH}$ \\
\hline tEME-AA & $17_{3,14}-16_{2,15}$ & 259137.860 & 69.5 & 4.97 & 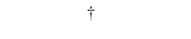 & & & \\
\hline tEME-EE & $19_{2,17}-18_{1,18}$ & 260106.865 & 78.5 & 2.13 & $\ldots$ & $\ldots$ & 0.05 & $\mathrm{CH}_{3} \mathrm{CH}_{2} \mathrm{OH}$ \\
\hline tEME-EE' & $19_{2,17}-18_{1,18}$ & 260106.865 & 78.5 & 2.13 & $\dagger$ & & & \\
\hline tEME-AE & $19_{2,17}-18_{1,18}$ & 260107.129 & 78.5 & 2.13 & $\dagger$ & & & \\
\hline tEME-EA & $19_{2,17}-18_{1,18}$ & 260108.541 & 78.5 & 2.13 & $\ldots$ & $\ldots$ & 0.05 & $\mathrm{CH}_{3} \mathrm{CH}_{2} \mathrm{OH}$ \\
\hline tEME-AA & $19_{2,17}-18_{1,18}$ & 260108.805 & 78.5 & 2.13 & $\dagger$ & & & \\
\hline tEME-EE' & $33_{1,33}-32_{0,32}$ & 260393.451 & 213.3 & 27.76 & $\ldots$ & $\ldots$ & 0.29 & $\mathrm{CH}_{3} \mathrm{OCOH}$ \\
\hline tEME-EE & $33_{1,33}-32_{0,32}$ & 260393.451 & 213.3 & 27.76 & $\dagger$ & & & \\
\hline tEME-AE & $33_{1,33}-32_{0,32}$ & 260393.460 & 213.3 & 27.76 & $\dagger$ & & & \\
\hline tEME-EA & $33_{1,33}-32_{0,32}$ & 260393.552 & 213.3 & 27.76 & $\dagger$ & & & \\
\hline tEME-AA & $33_{1,33}-32_{0,32}$ & 260393.560 & 213.3 & 27.76 & $\dagger$ & & & \\
\hline
\end{tabular}


A\&A 582, L1 (2015)

Table A.2. continued.

\begin{tabular}{|c|c|c|c|c|c|c|c|c|}
\hline Species & $\begin{array}{c}\text { Transition } \\
J_{K_{\mathrm{a}}, K_{\mathrm{c}}}-J_{K_{\mathrm{a}}^{\prime}, K_{\mathrm{c}}^{\prime}}^{\prime}\end{array}$ & $\begin{array}{c}\text { Predicted } \\
\text { frequency }(\mathrm{MHz})\end{array}$ & $\begin{array}{r}E_{\text {upp }} \\
(\mathrm{K})\end{array}$ & $S_{i j}$ & $\begin{array}{c}\text { Observed }^{1} \\
\text { frequency }(\mathrm{MHz})\end{array}$ & $\begin{array}{c}\text { Observed }^{1} \\
T_{\mathrm{MB}}(\mathrm{K})\end{array}$ & $\begin{array}{c}\text { Model }^{2} \\
T_{\mathrm{MB}}(\mathrm{K})\end{array}$ & Blends \\
\hline tEME-EE' & $40_{6,35}-40_{5,36}$ & 260504.644 & 358.5 & 20.96 & $\ldots$ & $\ldots$ & & $\mathrm{SiO}$ \\
\hline tEME-EE & $40_{6,35}-40_{5,36}$ & 260505.033 & 358.5 & 21.22 & $\dagger$ & & & \\
\hline tEME-AE & $40_{6,35}-40_{5,36}$ & 260505.210 & 358.5 & 21.10 & $\dagger$ & & & \\
\hline tEME-EA & $40_{6,35}-40_{5,36}$ & 260507.959 & 358.5 & 21.43 & $\dagger$ & & & \\
\hline tEME-AA & $40_{6,35}-40_{5,36}$ & 260508.333 & 358.5 & 21.45 & $\dagger$ & & & \\
\hline tEME-EE & $38_{6,32}-38_{5,33}$ & 260681.821 & 327.9 & 19.53 & $\ldots$ & $\ldots$ & 0.03 & $\mathrm{CH}_{3} \mathrm{CH}_{2} \mathrm{CN}$ \\
\hline tEME-AE & $38_{6,32}-38_{5,33}$ & 260682.506 & 327.9 & 19.20 & $\dddot{\dagger}$ & & & \\
\hline tEME-EE' & $38_{6,32}-38_{5,33}$ & 260682.515 & 327.9 & 18.84 & $\dot{\dagger}$ & & & \\
\hline tEME-EA & $38_{6,32}-38_{5,33}$ & 260683.965 & 327.9 & 20.17 & $\dagger$ & & & \\
\hline tEME-AA & $38_{6,32}-38_{5,33}$ & 260684.291 & 327.9 & 20.22 & $\dagger$ & & & \\
\hline tEME-EE & $37_{6,31}-37_{5,32}$ & 261001.915 & 313.2 & 18.45 & $\ldots$ & $\ldots$ & 0.03 & $\mathrm{OC}^{34} \mathrm{~S}, \mathrm{CH}_{3} \mathrm{OCOH}$ \\
\hline tEME-AE & $37_{6,31}-37_{5,32}$ & 261002.704 & 313.2 & 17.96 & $\dot{\dagger}$ & & & \\
\hline tEME-EE' & $37_{6,31}-37_{5,32}$ & 261002.807 & 313.2 & 17.46 & $\dagger$ & & & \\
\hline tEME-EE' & $38_{6,33}-38_{5,34}$ & 261029.561 & 327.9 & 18.84 & 261030.4 & 0.27 & 0.03 & $\mathrm{CH}_{3} \mathrm{OCOH} v_{t}=1$ \\
\hline tEME-EE & $38_{6,33}-38_{5,34}$ & 261030.255 & 327.9 & 19.53 & $\dagger$ & & & \\
\hline tEME-AE & $38_{6,33}-38_{5,34}$ & 261030.302 & 327.9 & 19.20 & $\dagger$ & & & \\
\hline tEME-EA & $38_{6,33}-38_{5,34}$ & 261033.470 & 327.9 & 20.16 & $\ldots$ & $\ldots$ & 0.03 & $\mathrm{CH}_{3} \mathrm{OCOH}$ \\
\hline tEME-AA & $38_{6,33}-38_{5,34}$ & 261033.876 & 327.9 & 20.22 & 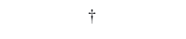 & & & \\
\hline tEME-EE' & $37_{6,32}-37_{5,33}$ & 261270.008 & 313.2 & 17.46 & $\ldots$ & $\ldots$ & 0.03 & $\mathrm{CH}_{3} \mathrm{CH}_{2} \mathrm{CN} v_{13} / v_{21}$ \\
\hline tEME-AE & $37_{6,32}-37_{5,33}$ & 261270.856 & 313.2 & 17.95 & $\dot{\dagger}$ & & & \\
\hline tEME-EE & $37_{6,32}-37_{5,33}$ & 261270.899 & 313.2 & 18.45 & $\dagger$ & & & \\
\hline tEME-AE & $36_{6,30}-36_{5,31}$ & 261291.812 & 298.9 & 16.50 & $\ldots$ & $\ldots$ & 0.04 & $\mathrm{CH}_{3} \mathrm{CH}_{2} \mathrm{OH}$ \\
\hline tEME-EE' & $36_{6,30}-36_{5,31}$ & 261292.008 & 298.9 & 15.92 & $\dagger$ & & & \\
\hline tEME-EA & $36_{6,30}-36_{5,31}$ & 261292.672 & 298.9 & 18.82 & $\dagger$ & & & \\
\hline tEME-AA & $36_{6,30}-36_{5,31}$ & 261292.970 & 298.9 & 19.00 & $\dagger$ & & & \\
\hline tEME-AE & $36_{6,31}-36_{5,32}$ & 261496.448 & 298.9 & 16.50 & $\ldots$ & $\ldots$ & 0.03 & $\mathrm{CH}_{2} \mathrm{CN}$ \\
\hline tEME-EE & $36_{6,31}-36_{5,32}$ & 261496.589 & 298.9 & 17.15 & $\dagger$ & & & \\
\hline tEME-EA & $36_{6,31}-36_{5,32}$ & 261500.259 & 298.9 & 18.82 & $\ldots$ & $\ldots$ & 0.03 & $\mathrm{CH}_{3} \mathrm{OCOH}, \mathrm{CH}_{2} \mathrm{CN}$ \\
\hline tEME-AA & $36_{6,31}-36_{5,32}$ & 261500.719 & 298.9 & 19.00 & $\dagger$ & & & \\
\hline tEME-EE' & $35_{6,29}-35_{5,30}$ & 261553.036 & 285.0 & 14.38 & 261553.2 & 0.15 & 0.06 & $\mathrm{CH}_{3} \mathrm{O}^{13} \mathrm{COH} v_{t}=1$ \\
\hline tEME-EA & $35_{6,29}-35_{5,30}$ & 261553.277 & 285.0 & 18.06 & $\dagger$ & & & \\
\hline tEME-AA & $35_{6,29}-35_{5,30}$ & 261553.546 & 285.0 & 18.40 & $\dagger$ & & & \\
\hline tEME-AE & $35_{6,30}-35_{5,31}$ & 261707.095 & 285.0 & 14.94 & $\ldots$ & $\ldots$ & 0.03 & $\mathrm{CH}_{3} \mathrm{OH}$ \\
\hline tEME-EE & $35_{6,30}-35_{5,31}$ & 261707.325 & 285.0 & 15.65 & $\dagger$ & & & \\
\hline tEME-EA & $35_{6,30}-35_{5,31}$ & 261711.277 & 285.0 & 18.06 & $\ldots$ & $\ldots$ & 0.03 & $\mathrm{CH}_{3} \mathrm{OCOH}$ \\
\hline tEME-AA & $35_{6,30}-35_{5,31}$ & 261711.778 & 285.0 & 18.40 & 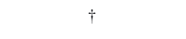 & & & \\
\hline tEME-EE & $34_{6,28}-34_{5,29}$ & 261787.091 & 271.4 & 14.12 & $\ldots$ & $\ldots$ & 0.07 & $\mathrm{CH}_{3} \mathrm{OCOH}, \mathrm{CH}_{3} \mathrm{OH}, \mathrm{SO}$ \\
\hline tEME-AE & $34_{6,28}-34_{5,29}$ & 261788.168 & 271.4 & 13.47 & $\dagger$ & & & \\
\hline tEME-EA & $34_{6,28}-34_{5,29}$ & 261788.351 & 271.4 & 17.16 & $\dagger$ & & & \\
\hline tEME-EE' & $34_{6,28}-34_{5,29}$ & 261788.486 & 271.4 & 13.02 & $\dagger$ & & & \\
\hline tEME-AA & $34_{6,28}-34_{5,29}$ & 261788.577 & 271.4 & 17.80 & $\dagger$ & & & \\
\hline tEME-EE' & $34_{6,29}-34_{5,30}$ & 261901.878 & 271.4 & 13.02 & $\ldots$ & $\ldots$ & 0.03 & $\mathrm{CH}_{3} \mathrm{OCH}_{3}, \mathrm{CH}_{3} \mathrm{COCH}_{3}$ \\
\hline tEME-AE & $34_{6,29}-34_{5,30}$ & 261902.977 & 271.4 & 13.47 & $\dddot{\dagger}$ & & & \\
\hline tEME-EE & $34_{6,29}-34_{5,30}$ & 261903.273 & 271.4 & 14.12 & $\dagger$ & & & \\
\hline tEME-EA & $34_{6,29}-34_{5,30}$ & 261907.508 & 271.4 & 17.16 & $\ldots$ & $\ldots$ & 0.03 & $\mathrm{CH}_{3} \mathrm{OCH}_{3}, \mathrm{CH}_{3} \mathrm{COCH}_{3}$ \\
\hline tEME-AA & $34_{6,29}-34_{5,30}$ & 261908.064 & 271.4 & 17.80 & $\dot{\dagger}$ & & & \\
\hline tEME-EA & $33_{6,27}-33_{5,29}$ & 262000.261 & 258.3 & 16.05 & $\ldots$ & $\ldots$ & 0.08 & $\mathrm{CCH}$ \\
\hline tEME-AE & $33_{6,27}-33_{5,29}$ & 262000.337 & 258.3 & 12.24 & $\dot{\dagger}$ & & & \\
\hline tEME-AA & $33_{6,27}-33_{5,29}$ & 262000.424 & 258.3 & 17.21 & $\dagger$ & & & \\
\hline tEME-EE' & $33_{6,27}-33_{5,29}$ & 262000.673 & 258.3 & 11.95 & $\dagger$ & & & \\
\hline tEME-AE & $33_{6,27}-33_{5,29}$ & 262084.400 & 258.3 & 12.24 & $\ldots$ & $\ldots$ & 0.03 & $\mathrm{CH}_{3} \mathrm{OCOH}$ \\
\hline tEME-EE & $33_{6,27}-33_{5,29}$ & 262084.731 & 258.3 & 12.72 & $\grave{\dagger}$ & & & \\
\hline tEME-EA & $33_{6,27}-33_{5,29}$ & 262089.211 & 258.3 & 16.05 & $\ldots$ & $\ldots$ & 0.03 & $\mathrm{CH}_{3} \mathrm{OCOH}$ \\
\hline tEME-AA & $33_{6,27}-33_{5,29}$ & 262089.841 & 258.3 & 17.21 & $\ddot{\dagger}$ & & & \\
\hline tEME-EA & $32_{6,27}-32_{5,28}$ & 262191.119 & 245.5 & 14.67 & $\ldots$ & $\ldots$ & 0.08 & $\mathrm{CH}_{3} \mathrm{CH}_{2} \mathrm{CN}$ \\
\hline tEME-AA & $32_{6,26}-32_{5,28}$ & 262191.200 & 245.5 & 16.62 & 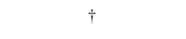 & & & \\
\hline tEME-AE & $32_{6,26}-32_{5,28}$ & 262191.354 & 245.5 & 11.34 & $\dagger$ & & & \\
\hline tEME-EE' & $32_{6,26}-32_{5,28}$ & 262191.682 & 245.5 & 11.22 & $\dagger$ & & & \\
\hline tEME-AE & $32_{6,27}-32_{5,28}$ & 262251.766 & 245.5 & 11.34 & $\ldots$ & $\ldots$ & 0.04 & $\mathrm{SO}_{2}$ \\
\hline tEME-EE & $32_{6,27}-32_{5,28}$ & 262252.104 & 245.5 & 11.61 & $\dagger$ & & & \\
\hline tEME-EA & $32_{6,27}-32_{5,28}$ & 262256.756 & 245.5 & 14.67 & $\ldots$ & $\ldots$ & 0.04 & $\mathrm{SO}_{2}$ \\
\hline tEME-AA & $32_{6,27}-32_{5,28}$ & 262257.479 & 245.5 & 16.62 & $\dot{\dagger}$ & & & \\
\hline tEME-AE & $32_{6,26}-32_{5,28}$ & 262260.269 & 245.5 & 5.28 & $\dagger$ & & & \\
\hline tEME-EE' & $32_{6,26}-32_{5,28}$ & 262260.849 & 245.5 & 5.41 & $\dagger$ & & & \\
\hline tEME-AA & $31_{6,25}-31_{5,26}$ & 262362.797 & 233.1 & 16.04 & $\ldots$ & $\ldots$ & 0.08 & $\mathrm{CH}_{3}{ }^{13} \mathrm{CH}_{2} \mathrm{CN}$ \\
\hline tEME-EA & $31_{6,25}-31_{5,26}$ & 262362.814 & 233.1 & 13.11 & $\dagger$ & & & \\
\hline tEME-AE & $31_{6,25}-31_{5,26}$ & 262363.092 & 233.1 & 10.77 & $\dot{\dagger}$ & & & \\
\hline tEME-EE' & $31_{6,25}-31_{5,26}$ & 262363.388 & 233.1 & 10.80 & $\dot{\dagger}$ & & & \\
\hline tEME-EE' & $31_{6,26}-31_{5,27}$ & 262404.436 & 233.1 & 10.80 & $\ldots$ & $\ldots$ & 0.04 & ${ }^{13} \mathrm{CH}_{3} \mathrm{OCOH} v_{t}=1$ \\
\hline tEME-AE & $31_{6,26}-31_{5,27}$ & 262405.547 & 233.1 & 10.77 & $\dot{\dagger}$ & & & \\
\hline tEME-EE & $31_{6,25}-31_{5,27}$ & 262405.867 & 233.1 & 10.84 & $\dagger$ & & & \\
\hline tEME-EA & $31_{6,26}-31_{5,27}$ & 262410.601 & 233.1 & 13.11 & $\ldots$ & $\ldots$ & 0.04 & ${ }^{13} \mathrm{CH}_{3} \mathrm{OCOH} v_{t}=1$ \\
\hline tEME-AA & $31_{6,26}-31_{5,27}$ & 262411.432 & 233.1 & 16.04 & 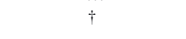 & & & \\
\hline
\end{tabular}


B. Tercero et al.: Trans ethyl methyl ether in space

Table A.2. continued.

\begin{tabular}{|c|c|c|c|c|c|c|c|c|}
\hline Species & $\begin{array}{c}\text { Transition } \\
J_{K_{\mathrm{a}}, K_{\mathrm{c}}}-J_{K_{\mathrm{a}}^{\prime}, K_{\mathrm{c}}^{\prime}}^{\prime}\end{array}$ & $\begin{array}{c}\text { Predicted } \\
\text { frequency }(\mathrm{MHz})\end{array}$ & $\begin{array}{r}E_{\text {upp }} \\
(\mathrm{K})\end{array}$ & $S_{i j}$ & $\begin{array}{c}\text { Observed }^{1} \\
\text { frequency }(\mathrm{MHz})\end{array}$ & $\begin{array}{c}\text { Observed }^{1} \\
T_{\mathrm{MB}}(\mathrm{K})\end{array}$ & $\begin{array}{c}\text { Model }^{2} \\
T_{\mathrm{MB}}(\mathrm{K})\end{array}$ & Blends \\
\hline tEME-EA & $30_{6,25}-30_{5,25}$ & 262515.014 & 221.2 & 3.88 & $\ldots$ & $\ldots$ & 0.09 & U-line \\
\hline tEME-EE & $30_{6,24}-30_{5,25}$ & 262516.140 & 221.2 & 10.39 & $\dagger$ & & & \\
\hline tEME-AA & $30_{6,24}-30_{5,25}$ & 262516.918 & 221.2 & 15.45 & $\dagger$ & & & \\
\hline tEME-EA & $30_{6,24}-30_{5,25}$ & 262517.034 & 221.2 & 11.57 & $\dagger$ & & & \\
\hline tEME-AE & $30_{6,24}-30_{5,25}$ & 262517.242 & 221.2 & 10.51 & $\dagger$ & & & \\
\hline tEME-EE' & $30_{6,24}-30_{5,25}$ & 262517.483 & 221.2 & 10.68 & $\dagger$ & & & \\
\hline tEME-EE' & $30_{6,25}-30_{5,26}$ & 262545.215 & 221.2 & 10.68 & $\ldots$ & $\ldots$ & 0.04 & $\mathrm{H}_{2} \mathrm{CCO}$ \\
\hline tEME-AE & $30_{6,25}-30_{5,26}$ & 262546.281 & 221.2 & 10.51 & 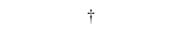 & & & \\
\hline tEME-EE & $30_{6,25}-30_{5,26}$ & 262546.558 & 221.2 & 10.39 & $\dagger$ & & & \\
\hline tEME-EA & $30_{6,25}-30_{5,26}$ & 262551.283 & 221.2 & 11.57 & $\ldots$ & $\ldots$ & 0.04 & $\mathrm{H}_{2} \mathrm{CCO}$ \\
\hline tEME-AA & $30_{6,25}-30_{5,26}$ & 262552.224 & 221.2 & 15.45 & $\dagger$ & & & \\
\hline tEME-EE & $30_{6,24}-30_{5,26}$ & 262553.207 & 221.2 & 5.06 & $\dagger$ & & & \\
\hline tEME-EA & $30_{6,24}-30_{5,26}$ & 262553.303 & 221.2 & 3.88 & $\dagger$ & & & \\
\hline tEME-EE & $29_{6,23}-29_{5,24}$ & 262654.283 & 209.6 & 10.25 & $\ldots$ & $\ldots$ & 0.10 & $\mathrm{CH}_{3} \mathrm{COCH}_{3}$ \\
\hline tEME-AA & $29_{6,23}-29_{5,24}$ & 262655.095 & 209.6 & 14.87 & 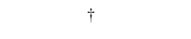 & & & \\
\hline tEME-EA & $29_{6,23}-29_{5,24}$ & 262655.300 & 209.6 & 10.29 & $\dagger$ & & & \\
\hline tEME-AE & $29_{6,23}-29_{5,24}$ & 262655.326 & 209.6 & 10.53 & $\dagger$ & & & \\
\hline tEME-EE' & $29_{6,23}-29_{5,24}$ & 262655.485 & 209.6 & 10.83 & $\dagger$ & & & \\
\hline tEME-EE' & $29_{6,24}-29_{5,25}$ & 262673.561 & 209.6 & 10.83 & $\ldots$ & $\ldots$ & 0.05 & $\mathrm{HC}^{13} \mathrm{CCN}$ \\
\hline tEME-AE & $29_{6,24}-29_{5,25}$ & 262674.556 & 209.6 & 10.53 & $\dot{\dagger}$ & & & \\
\hline tEME-EE & $29_{6,24}-29_{5,25}$ & 262674.763 & 209.6 & 10.25 & $\dagger$ & & & \\
\hline tEME-EA & $29_{6,24}-29_{5,25}$ & 262679.394 & 209.6 & 10.29 & $\ldots$ & $\ldots$ & 0.04 & $\mathrm{HC}^{13} \mathrm{CCN}$ \\
\hline tEME-AA & $29_{6,24}-29_{5,25}$ & 262680.434 & 209.6 & 14.87 & $\dagger$ & & & \\
\hline tEME-EA & $29_{6,23}-29_{5,25}$ & 262681.315 & 209.6 & 4.58 & $\dagger$ & & & \\
\hline tEME-EE & $29_{6,23}-29_{5,25}$ & 262681.410 & 209.6 & 4.63 & $\dagger$ & & & \\
\hline tEME-EA & $28_{6,23}-28_{5,23}$ & 262777.103 & 198.3 & 4.94 & $\ldots$ & $\ldots$ & 0.10 & $\mathrm{CH}_{3} \mathrm{OCH}_{3}$ \\
\hline tEME-EE & $28_{6,22}-28_{5,23}$ & 262777.763 & 198.3 & 10.37 & $\dagger$ & & & \\
\hline tEME-AA & $28_{6,22}-28_{5,23}$ & 262778.714 & 198.3 & 14.30 & $\dagger$ & & & \\
\hline tEME-AE & $28_{6,22}-28_{5,23}$ & 262778.719 & 198.3 & 10.78 & $\dagger$ & & & \\
\hline tEME-EE’ & $28_{6,22}-28_{5,23}$ & 262778.771 & 198.3 & 11.17 & $\dagger$ & & & \\
\hline tEME-EA & $28_{6,22}-28_{5,23}$ & 262778.988 & 198.3 & 9.35 & $\dagger$ & & & \\
\hline tEME-EE' & $28_{6,23}-28_{5,24}$ & 262790.103 & 198.3 & 11.17 & 262791.0 & 0.25 & 0.05 & U-line \\
\hline tEME-AE & $28_{6,23}-28_{5,24}$ & 262791.000 & 198.3 & 10.78 & $\dagger$ & & & \\
\hline tEME-EE & $28_{6,23}-28_{5,24}$ & 262791.111 & 198.3 & 10.37 & $\dagger$ & & & \\
\hline tEME-EA & $28_{6,23}-28_{5,24}$ & 262795.561 & 198.3 & 9.35 & $\ldots$ & $\ldots$ & 0.04 & $\mathrm{CH}_{2} \mathrm{OHCHO}$ \\
\hline tEME-AA & $28_{6,23}-28_{5,24}$ & 262796.681 & 198.3 & 14.30 & $\dagger$ & & & \\
\hline tEME-EA & $28_{6,22}-28_{5,24}$ & 262797.446 & 198.3 & 4.94 & $\dagger$ & & & \\
\hline tEME-EE & $28_{6,22}-28_{5,24}$ & 262797.772 & 198.3 & 3.92 & $\dagger$ & & & \\
\hline tEME-EA & $27_{6,22}-27_{5,22}$ & 262887.474 & 187.5 & 4.97 & $\ldots$ & $\ldots$ & 0.10 & $\mathrm{CH}_{3} \mathrm{COOH}, \mathrm{CH}_{3} \mathrm{OCH}_{3}$ \\
\hline tEME-EE & $27_{6,21}-27_{5,22}$ & 262887.831 & 187.5 & 10.70 & $\dagger$ & & & \\
\hline tEME-EE' & $27_{6,21}-27_{5,22}$ & 262888.603 & 187.5 & 11.58 & $\dagger$ & & & \\
\hline tEME-AE & $27_{6,21}-27_{5,22}$ & 262888.676 & 187.5 & 11.18 & $\dagger$ & & & \\
\hline tEME-AA & $27_{6,21}-27_{5,22}$ & 262889.035 & 187.5 & 13.72 & $\dagger$ & & & \\
\hline tEME-EA & $27_{6,21}-27_{5,22}$ & 262889.353 & 187.5 & 8.75 & $\dagger$ & & & \\
\hline tEME-EE' & $27_{6,22}-27_{5,23}$ & 262895.483 & 187.5 & 11.58 & $\ldots$ & $\ldots$ & 0.07 & $\mathrm{CH}_{3} \mathrm{OCH}_{3}$ \\
\hline tEME-EE & $27_{6,22}-27_{5,23}$ & 262896.256 & 187.5 & 10.70 & $\dagger$ & & & \\
\hline tEME-AE & $27_{6,22}-27_{5,23}$ & 262896.265 & 187.5 & 11.18 & $\dagger$ & & & \\
\hline tEME-EA & $27_{6,22}-27_{5,23}$ & 262900.436 & 187.5 & 8.75 & $\ldots$ & $\ldots$ & 0.05 & $\mathrm{CH}_{3} \mathrm{COOH}$ \\
\hline tEME-AA & $27_{6,22}-27_{5,23}$ & 262901.609 & 187.5 & 13.72 & $\dagger$ & & & \\
\hline tEME-EA & $27_{6,21}-27_{5,23}$ & 262902.316 & 187.5 & 4.97 & $\dagger$ & & & \\
\hline tEME-EE & $27_{6,21}-27_{5,23}$ & 262902.939 & 187.5 & 3.02 & $\dagger$ & & & \\
\hline tEME-EE & $26_{6,20}-26_{5,21}$ & 262985.638 & 177.1 & 11.11 & $\ldots$ & $\ldots$ & 0.10 & $\mathrm{CH}_{3}{ }^{18} \mathrm{OH}$ \\
\hline tEME-EA & $26_{6,21}-26_{5,21}$ & 262985.653 & 177.1 & 4.68 & $\dagger$ & & & \\
\hline tEME-EE' & $26_{6,20}-26_{5,21}$ & 262986.169 & 177.1 & 11.87 & $\dagger$ & & & \\
\hline tEME-AE & $26_{6,20}-26_{5,21}$ & 262986.363 & 177.1 & 11.54 & $\dagger$ & & & \\
\hline tEME-AA & $26_{6,20}-26_{5,21}$ & 262987.205 & 177.1 & 13.15 & $\dagger$ & & & \\
\hline tEME-EA & $26_{6,20}-26_{5,21}$ & 262987.541 & 177.1 & 8.47 & $\dagger$ & & & \\
\hline tEME-EE' & $26_{6,21}-26_{5,22}$ & 262990.328 & 177.1 & 11.87 & $\ldots$ & $\ldots$ & 0.09 & $\mathrm{CH}_{3}{ }^{18} \mathrm{OH}$ \\
\hline tEME-EE & $26_{6,21}-26_{5,22}$ & 262990.859 & 177.1 & 11.11 & $\dagger$ & & & \\
\hline tEME-AE & $26_{6,21}-26_{5,22}$ & 262990.997 & 177.1 & 11.54 & $\dagger$ & & & \\
\hline tEME-EA & $26_{6,21}-26_{5,22}$ & 262994.684 & 177.1 & 8.47 & $\ldots$ & $\ldots$ & 0.05 & $\mathrm{SO}_{2} v_{2}=1$ \\
\hline tEME-AA & $26_{6,21}-26_{5,22}$ & 262995.883 & 177.1 & 13.15 & $\dagger$ & & & \\
\hline tEME-EA & $26_{6,20}-26_{5,22}$ & 262996.572 & 177.1 & 4.68 & $\dagger$ & & & \\
\hline tEME-EE & $26_{6,20}-26_{5,22}$ & 262997.567 & 177.1 & 2.04 & $\dagger$ & & & \\
\hline tEME-EE & $25_{6,19}-25_{5,20}$ & 263072.263 & 167.0 & 11.39 & $\ldots$ & $\ldots$ & 0.11 & U-line \\
\hline tEME-EE' & $25_{6,19}-25_{5,20}$ & 263072.594 & 167.0 & 11.92 & $\dagger$ & & & \\
\hline tEME-EA & $25_{6,20}-25_{5,20}$ & 263072.699 & 167.0 & 4.12 & $\dagger$ & & & \\
\hline tEME-AE & $25_{6,19}-25_{5,20}$ & 263072.885 & 167.0 & 11.70 & $\dagger$ & & & \\
\hline tEME-AA & $25_{6,19}-25_{5,20}$ & 263074.277 & 167.0 & 12.58 & $\ldots$ & $\ldots$ & 0.11 & U-line \\
\hline tEME-EA & $25_{6,19}-25_{5,20}$ & 263074.602 & 167.0 & 8.46 & $\dagger$ & & & \\
\hline tEME-EE' & $25_{6,20}-25_{5,21}$ & 263075.234 & 167.0 & 11.92 & $\dagger$ & & & \\
\hline tEME-EE & $25_{6,20}-25_{5,21}$ & 263075.564 & 167.0 & 11.39 & $\dagger$ & & & \\
\hline tEME-AE & $25_{6,20}-25_{5,21}$ & 263075.815 & 167.0 & 11.70 & $\dagger$ & & & \\
\hline
\end{tabular}


A\&A 582, L1 (2015)

Table A.2. continued.

\begin{tabular}{|c|c|c|c|c|c|c|c|c|}
\hline Species & $\begin{array}{c}\text { Transition } \\
J_{K_{\mathrm{a}}, K_{\mathrm{c}}}-J_{K_{\mathrm{a}}^{\prime}, K_{\mathrm{c}}^{\prime}}^{\prime}\end{array}$ & $\begin{array}{c}\text { Predicted } \\
\text { frequency }(\mathrm{MHz})\end{array}$ & $\begin{array}{r}E_{\text {upp }} \\
(\mathrm{K})\end{array}$ & $S_{i j}$ & $\begin{array}{c}\text { Observed }^{1} \\
\text { frequency }(\mathrm{MHz})\end{array}$ & $\begin{array}{c}\text { Observed }^{1} \\
T_{\mathrm{MB}}(\mathrm{K})\end{array}$ & $\begin{array}{c}\text { Model }^{2} \\
T_{\mathrm{MB}}(\mathrm{K})\end{array}$ & Blends \\
\hline tEME-EA & $25_{6,20}-25_{5,21}$ & 263078.980 & 167.0 & 8.46 & $\ldots$ & $\ldots$ & 0.05 & U-line \\
\hline tEME-AA & $25_{6,20}-25_{5,21}$ & 263080.176 & 167.0 & 12.58 & $\dagger$ & & & \\
\hline tEME-EE & $24_{6,19}-24_{5,19}$ & 263141.974 & 157.4 & 0.60 & 263149.1 & 0.44 & 0.11 & $\mathrm{CH}_{3} \mathrm{O}^{13} \mathrm{COH}, \mathrm{CH}_{3} \mathrm{OCOD}$ \\
\hline tEME-EE & $24_{6,18}-24_{5,19}$ & 263148.731 & 157.4 & 11.41 & $\dagger$ & & & \\
\hline tEME-EE' & $24_{6,18}-24_{5,19}$ & 263148.928 & 157.4 & 11.70 & $\dagger$ & & & \\
\hline tEME-AE & $24_{6,18}-24_{5,19}$ & 263149.281 & 157.4 & 11.59 & $\dagger$ & & & \\
\hline tEME-EA & $24_{6,19}-24_{5,19}$ & 263149.579 & 157.4 & 3.30 & $\dagger$ & & & \\
\hline tEME-EE' & $24_{6,19}-24_{5,20}$ & 263150.791 & 157.4 & 11.70 & 263151.0 & 0.58 & 0.20 & $\mathrm{CH}_{3} \mathrm{O}^{13} \mathrm{COH}, \mathrm{CH}_{3} \mathrm{OCOD}$ \\
\hline tEME-EE & $24_{6,19}-24_{5,20}$ & 263150.988 & 157.4 & 11.41 & $\dagger$ & & & \\
\hline tEME-AA & $24_{6,18}-24_{5,19}$ & 263151.216 & 157.4 & 12.01 & $\dagger$ & & & \\
\hline tEME-AE & $24_{6,19}-24_{5,20}$ & 263151.318 & 157.4 & 11.59 & $\dagger$ & & & \\
\hline tEME-EA & $24_{6,18}-24_{5,19}$ & 263151.498 & 157.4 & 8.71 & $\dagger$ & & & \\
\hline tEME-EA & $24_{6,19}-24_{5,20}$ & 263153.999 & 157.4 & 8.71 & $\dagger$ & & & \\
\hline tEME-AA & $24_{6,19}-24_{5,20}$ & 263155.161 & 157.4 & 12.01 & 263155.2 & 0.25 & 0.05 & $\mathrm{CH}_{3} \mathrm{OCOD}$ \\
\hline tEME-EA & $24_{6,18}-24_{5,20}$ & 263155.918 & 157.4 & 3.30 & $\dagger$ & & & \\
\hline tEME-EE & $23_{6,17}-23_{5,18}$ & 263215.996 & 148.1 & 11.17 & 263216.3 & 0.58 & 0.14 & $\mathrm{SO}_{2}$ \\
\hline tEME-EE' & $23_{6,17}-23_{5,18}$ & 263216.118 & 148.1 & 11.31 & $\grave{\dagger}$ & & & \\
\hline tEME-AE & $23_{6,17}-23_{5,18}$ & 263216.506 & 148.1 & 11.26 & $\dagger$ & & & \\
\hline tEME-EA & $23_{6,18}-23_{5,18}$ & 263217.183 & 148.1 & 2.31 & 263217.8 & 0.59 & 0.14 & $\mathrm{SO}_{2}$ \\
\hline tEME-EE' & $23_{6,18}-23_{5,19}$ & 263217.615 & 148.1 & 11.31 & $\doteqdot$ & & & \\
\hline tEME-EE & $23_{6,18}-23_{5,19}$ & 263217.736 & 148.1 & 11.17 & $\dagger$ & & & \\
\hline tEME-AE & $23_{6,18}-23_{5,19}$ & 263218.114 & 148.1 & 11.26 & $\dagger$ & & & \\
\hline tEME-AA & $23_{6,17}-23_{5,18}$ & 263218.914 & 148.1 & 11.44 & $\dagger$ & & & \\
\hline tEME-EA & $23_{6,17}-23_{5,18}$ & 263219.118 & 148.1 & 9.14 & $\dagger$ & & & \\
\hline tEME-EA & $23_{6,18}-23_{5,19}$ & 263220.415 & 148.1 & 9.14 & $\ldots$ & $\ldots$ & 0.05 & $\mathrm{SO}_{2}$ \\
\hline tEME-AA & $23_{6,18}-23_{5,19}$ & 263221.507 & 148.1 & 11.44 & $\dot{\dagger}$ & & & \\
\hline tEME-EA & $23_{6,17}-23_{5,19}$ & 263222.351 & 148.1 & 2.31 & $\dagger$ & & & \\
\hline tEME-EE & $22_{6,16}-22_{5,17}$ & 263274.922 & 139.2 & 10.76 & $\ldots$ & $\ldots$ & 0.14 & $\mathrm{CH}_{3} \mathrm{OCH}_{3}$ \\
\hline tEME-EE' & $22_{6,16}-22_{5,17}$ & 263275.007 & 139.2 & 10.82 & $\dagger$ & & & \\
\hline tEME-AE & $22_{6,16}-22_{5,17}$ & 263275.415 & 139.2 & 10.80 & $\dot{\dagger}$ & & & \\
\hline tEME-EA & $22_{6,17}-22_{5,17}$ & 263276.341 & 139.2 & 1.34 & $\dagger$ & & & \\
\hline tEME-EE' & $22_{6,17}-22_{5,18}$ & 263276.343 & 139.2 & 10.82 & $\dagger$ & & & \\
\hline tEME-EE & $22_{6,17}-22_{5,18}$ & 263276.429 & 139.2 & 10.76 & $\dagger$ & & & \\
\hline tEME-AE & $22_{6,17}-22_{5,18}$ & 263276.831 & 139.2 & 10.80 & $\dagger$ & & & \\
\hline tEME-AA & $22_{6,16}-22_{5,17}$ & 263278.199 & 139.2 & 10.88 & $\ldots$ & $\ldots$ & 0.11 & $\mathrm{CH}_{3} \mathrm{OCH}_{3}$ \\
\hline tEME-EA & $22_{6,16}-22_{5,17}$ & 263278.292 & 139.2 & 9.53 & $\grave{\dagger}$ & & & \\
\hline tEME-EA & $22_{6,17}-22_{5,18}$ & 263278.882 & 139.2 & 9.53 & $\dagger$ & & & \\
\hline tEME-AA & $22_{6,17}-22_{5,18}$ & 263279.871 & 139.2 & 10.88 & $\dagger$ & & & \\
\hline tEME-EE & $21_{6,15}-21_{5,16}$ & 263326.288 & 130.7 & 10.27 & 263326.7 & 0.42 & 0.14 & $\mathrm{HC}_{3} \mathrm{~N} v_{7}=1, \mathrm{CH}_{3} \mathrm{COCH}_{3}$ \\
\hline tEME-EE' & $21_{6,15}-21_{5,16}$ & 263326.357 & 130.7 & 10.29 & $\doteqdot$ & & & \\
\hline tEME-AE & $21_{6,15}-21_{5,16}$ & 263326.775 & 130.7 & 10.28 & $\dot{\dagger}$ & & & \\
\hline tEME-EE' & $21_{6,16}-21_{5,17}$ & 263327.628 & 130.7 & 10.29 & 263327.4 & 0.51 & 0.14 & $\mathrm{HC}_{3} \mathrm{~N} v_{7}=1, \mathrm{CH}_{3} \mathrm{COCH}_{3}$ \\
\hline tEME-EE & $21_{6,16}-21_{5,17}$ & 263327.697 & 130.7 & 10.27 & $\dagger$ & & & \\
\hline tEME-EA & $21_{6,16}-21_{5,16}$ & 263327.838 & 130.7 & .64 & $\dagger$ & & & \\
\hline tEME-AE & $21_{6,16}-21_{5,17}$ & 263328.113 & 130.7 & 10.28 & $\dagger$ & & & \\
\hline tEME-EA & $21_{6,15}-21_{5,16}$ & 263329.805 & 130.7 & 9.67 & 263329.9 & 0.74 & 0.30 & $\mathrm{HC}_{3} \mathrm{~N} v_{7}=1, \mathrm{CH}_{3} \mathrm{COCH}_{3}$ \\
\hline tEME-AA & $21_{6,15}-21_{5,16}$ & 263329.839 & 130.7 & 10.31 & $\div$ & & & \\
\hline tEME-EA & $21_{6,16}-21_{5,17}$ & 263330.025 & 130.7 & 9.67 & $\dagger$ & & & \\
\hline tEME-AA & $21_{6,16}-21_{5,17}$ & 263330.894 & 130.7 & 10.31 & $\dagger$ & & & \\
\hline tEME-EE & $20_{6,14}-20_{5,15}$ & 263370.802 & 122.6 & 9.73 & 263370.7 & 0.51 & 0.16 & $\mathrm{SO}^{18} \mathrm{O}, \mathrm{CH}_{3} \mathrm{OCOH}$ \\
\hline tEME-EE' & $20_{6,14}-20_{5,15}$ & 263370.865 & 122.6 & 9.74 & $\dagger$ & & & \\
\hline tEME-AE & $20_{6,14}-20_{5,15}$ & 263371.289 & 122.6 & 9.73 & $\dagger$ & & & \\
\hline tEME-EE' & $20_{6,15}-20_{5,16}$ & 263372.112 & 122.6 & 9.74 & 263372.2 & 0.37 & 0.16 & $\mathrm{SO}^{18} \mathrm{O}, \mathrm{CH}_{3} \mathrm{OCOH}$ \\
\hline tEME-EE & $20_{6,15}-20_{5,16}$ & 263372.174 & 122.6 & 9.73 & $\dot{\dagger}$ & & & \\
\hline tEME-AE & $20_{6,15}-20_{5,16}$ & 263372.598 & 122.6 & 9.73 & $\dagger$ & & & \\
\hline tEME-EA & $20_{6,14}-20_{5,15}$ & 263374.395 & 122.6 & 9.49 & $\ldots$ & $\ldots$ & 0.17 & $\mathrm{SO}^{18} \mathrm{O}, \mathrm{CH}_{3} \mathrm{OCOH}$ \\
\hline tEME-EA & $20_{6,15}-20_{5,16}$ & 263374.447 & 122.6 & 9.49 & $\ddot{\dagger}$ & & & \\
\hline tEME-AA & $20_{6,14}-20_{5,15}$ & 263374.550 & 122.6 & 9.74 & $\dagger$ & & & \\
\hline tEME-AA & $20_{6,15}-20_{5,16}$ & 263375.201 & 122.6 & 9.74 & $\dagger$ & & & \\
\hline tEME-EE & $19_{6,13}-20_{5,14}$ & 263409.117 & 114.8 & 9.17 & $\ldots$ & $\ldots$ & 0.17 & $\mathrm{CH}_{3} \mathrm{OCH}_{3}$ \\
\hline tEME-EE' & $19_{6,13}-20_{5,14}$ & 263409.177 & 114.8 & 9.17 & $\dot{\dagger}$ & & & \\
\hline tEME-AE & $19_{6,13}-20_{5,14}$ & 263409.605 & 114.8 & 9.17 & $\dagger$ & & & \\
\hline tEME-EE' & $19_{6,14}-20_{5,15}$ & 263410.419 & 114.8 & 9.17 & $\dagger$ & & & \\
\hline tEME-EE & $19_{6,14}-20_{5,15}$ & 263410.479 & 114.8 & 9.17 & $\dagger$ & & & \\
\hline tEME-AE & $19_{6,14}-20_{5,15}$ & 263410.907 & 114.8 & 9.17 & $\dagger$ & & & \\
\hline tEME-EA & $19_{6,14}-20_{5,15}$ & 263412.731 & 114.8 & 9.09 & $\ldots$ & $\ldots$ & 0.19 & $\mathrm{CH}_{3} \mathrm{OCH}_{3}$ \\
\hline tEME-EA & $19_{6,13}-20_{5,14}$ & 263412.749 & 114.8 & 9.09 & $\dot{\dagger}$ & & & \\
\hline tEME-AA & $19_{6,13}-20_{5,14}$ & 263413.003 & 114.8 & 9.18 & $\dagger$ & & & \\
\hline tEME-EE & $18_{6,12}-18_{5,13}$ & 263441.843 & 107.5 & 8.61 & $\ldots$ & $\ldots$ & 0.17 & ${ }^{34} \mathrm{SO}_{2},{ }^{33} \mathrm{SO}_{2}$ \\
\hline tEME-EE' & $18_{6,12}-18_{5,13}$ & 263441.902 & 107.5 & 8.61 & $\dot{\dagger}$ & & & \\
\hline tEME-AE & $18_{6,12}-18_{5,13}$ & 263442.334 & 107.5 & 8.61 & $\dagger$ & & & \\
\hline tEME-EE' & $18_{6,13}-18_{5,14}$ & 263443.145 & 107.5 & 8.61 & $\dagger$ & & & \\
\hline tEME-EE & $18_{6,13}-18_{5,14}$ & 263443.204 & 107.5 & 8.61 & $\dagger$ & & & \\
\hline
\end{tabular}


B. Tercero et al.: Trans ethyl methyl ether in space

Table A.2. continued.

\begin{tabular}{|c|c|c|c|c|c|c|c|c|}
\hline Species & $\begin{array}{c}\text { Transition } \\
J_{K_{\mathrm{a}}, K_{\mathrm{c}}}-J_{K_{\mathrm{a}}^{\prime}, K_{\mathrm{c}}^{\prime}}^{\prime}\end{array}$ & $\begin{array}{c}\text { Predicted } \\
\text { frequency }(\mathrm{MHz})\end{array}$ & $\begin{array}{r}E_{\text {upp }} \\
(\mathrm{K})\end{array}$ & $S_{i j}$ & $\begin{array}{c}\text { Observed }^{1} \\
\text { frequency }(\mathrm{MHz})\end{array}$ & $\begin{array}{c}\text { Observed }^{1} \\
T_{\mathrm{MB}}(\mathrm{K})\end{array}$ & $\begin{array}{c}\text { Model }^{2} \\
T_{\mathrm{MB}}(\mathrm{K})\end{array}$ & Blends \\
\hline tEME-AE & $18_{6,13}-18_{5,14}$ & 263443.636 & 107.5 & 8.61 & $\bar{\dagger}$ & & & \\
\hline tEME-EA & $18_{6,13}-18_{5,14}$ & 263445.453 & 107.5 & 8.58 & $\ldots$ & $\ldots$ & 0.19 & ${ }^{34} \mathrm{SO}_{2},{ }^{33} \mathrm{SO}_{2}$ \\
\hline tEME-EA & $18_{6,12}-18_{5,13}$ & 263445.498 & 107.5 & 8.58 & $\dagger$ & & & \\
\hline tEME-AA & $18_{6,12}-18_{5,13}$ & 263445.822 & 107.5 & 8.61 & $\dagger$ & & & \\
\hline tEME-AA & $18_{6,13}-18_{5,14}$ & 263446.051 & 107.5 & 8.61 & $\dagger$ & & & \\
\hline tEME-EE & $17_{6,11}-17_{5,12}$ & 263469.553 & 100.5 & 8.04 & 263469.7 & 0.43 & 0.17 & $\mathrm{CH}_{3} \mathrm{CH}_{2} \mathrm{OH}$ \\
\hline tEME-EE' & $17_{6,11}-17_{5,12}$ & 263469.612 & 100.5 & 8.04 & $\dagger$ & & & \\
\hline tEME-AE & $17_{6,11}-17_{5,12}$ & 263470.047 & 100.5 & 8.04 & $\dagger$ & & & \\
\hline tEME-EE' & $17_{6,12}-17_{5,13}$ & 263470.859 & 100.5 & 8.04 & $\dagger$ & & & \\
\hline tEME-EE & $17_{6,12}-17_{5,13}$ & 263470.917 & 100.5 & 8.04 & $\dagger$ & & & \\
\hline tEME-AE & $17_{6,12}-17_{5,13}$ & 263471.352 & 100.5 & 8.04 & $\dagger$ & & & \\
\hline tEME-EA & $17_{6,12}-17_{5,13}$ & 263473.169 & 100.5 & 8.03 & 263473.9 & 0.41 & 0.19 & $\mathrm{CH}_{3} \mathrm{CH}_{2} \mathrm{OH}$ \\
\hline tEME-EA & $17_{6,11}-17_{5,12}$ & 263473.223 & 100.5 & 8.03 & $\dagger$ & & & \\
\hline tEME-AA & $17_{6,11}-17_{5,12}$ & 263473.595 & 100.5 & 8.04 & $\dagger$ & & & \\
\hline tEME-AA & $17_{6,12}-17_{5,13}$ & 263473.725 & 100.5 & 8.04 & $\dagger$ & & & \\
\hline tEME-EE & $16_{6,10}-16_{5,11}$ & 263492.786 & 94.0 & 7.46 & 263492.9 & 0.26 & 0.17 & $\mathrm{CH}_{3} \mathrm{OCH}_{3}$ \\
\hline tEME-EE' & $16_{6,10}-16_{5,11}$ & 263492.845 & 94.0 & 7.46 & $\dagger$ & & & \\
\hline tEME-AE & $16_{6,10}-16_{5,11}$ & 263493.283 & 94.0 & 7.46 & $\dagger$ & & & \\
\hline tEME-EE' & $16_{6,11}-16_{5,12}$ & 263494.096 & 94.0 & 7.46 & 263494.2 & 0.33 & 0.17 & $\mathrm{CH}_{3} \mathrm{OCH}_{3}$ \\
\hline tEME-EE & $16_{6,11}-16_{5,12}$ & 263494.155 & 94.0 & 7.46 & $\dagger$ & & & \\
\hline tEME-AE & $16_{6,11}-16_{5,12}$ & 263494.592 & 94.0 & 7.46 & $\dagger$ & & & \\
\hline tEME-EA & $16_{6,11}-16_{5,12}$ & 263496.411 & 94.0 & 7.46 & 263496.6 & 0.55 & 0.19 & $\mathrm{CH}_{3} \mathrm{OCH}_{3}$ \\
\hline tEME-EA & $16_{6,10}-16_{5,11}$ & 263496.468 & 94.0 & 7.46 & $\dagger$ & & & \\
\hline tEME-AA & $16_{6,10}-16_{5,11}$ & 263496.871 & 94.0 & 7.46 & $\dagger$ & & & \\
\hline tEME-AA & $16_{6,11}-16_{5,12}$ & 263496.942 & 94.0 & 7.46 & $\dagger$ & & & \\
\hline tEME-EE & $15_{6,9}-15_{5,10}$ & 263512.049 & 87.8 & 6.89 & $\ldots$ & $\ldots$ & 0.17 & $\mathrm{CH}_{3} \mathrm{OCH}_{3}$ \\
\hline tEME-EE' & $15_{6,9}-15_{5,10}$ & 263512.108 & 87.8 & 6.89 & $\dddot{\dagger}$ & & & \\
\hline tEME-AE & $15_{6,9}-15_{5,10}$ & 263512.548 & 87.8 & 6.89 & $\dagger$ & & & \\
\hline tEME-EE' & $15_{6,10}-15_{5,11}$ & 263513.363 & 87.8 & 6.89 & $\ldots$ & $\ldots$ & 0.17 & $\mathrm{CH}_{3} \mathrm{OCH}_{3}$ \\
\hline tEME-EE & $15_{6,10}-15_{5,11}$ & 263513.422 & 87.8 & 6.89 & $\dagger$ & & & \\
\hline tEME-AE & $15_{6,10}-15_{5,10}$ & 263513.862 & 87.8 & 6.89 & $\dagger$ & & & \\
\hline tEME-EA & $15_{6,10}-15_{5,11}$ & 263515.683 & 87.8 & 6.88 & $\ldots$ & $\ldots$ & 0.19 & $\mathrm{CH}_{3} \mathrm{CH}_{2} \mathrm{CN}$ \\
\hline tEME-EA & $15_{6,9}-15_{5,10}$ & 263515.742 & 87.8 & 6.88 & $\dagger$ & & & \\
\hline tEME-AA & $15_{6,9}-15_{5,10}$ & 263516.163 & 87.8 & 6.89 & $\dagger$ & & & \\
\hline tEME-AA & $15_{6,10}-15_{5,11}$ & 263516.200 & 87.8 & 6.89 & $\dagger$ & & & \\
\hline tEME-EE & $14_{6,8}-14_{5,9}$ & 263527.818 & 82.0 & 6.30 & $\ldots$ & $\ldots$ & 0.16 & $\mathrm{CH}_{3} \mathrm{OCH}_{3}, \mathrm{CH}_{3} \mathrm{CH}_{2} \mathrm{CN}$ \\
\hline tEME-EE' & $14_{6,8}-14_{5,9}$ & 263527.877 & 82.0 & 6.30 & 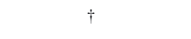 & & & \\
\hline tEME-AE & $14_{6,8}-14_{5,9}$ & 263528.319 & 82.0 & 6.30 & $\dagger$ & & & \\
\hline tEME-EE' & $14_{6,9}-14_{5,10}$ & 263529.136 & 82.0 & 6.30 & $\dagger$ & & & \\
\hline tEME-EE & $14_{6,9}-14_{5,10}$ & 263529.195 & 82.0 & 6.30 & $\dagger$ & & & \\
\hline tEME-AE & $14_{6,9}-14_{5,10}$ & 263529.637 & 82.0 & 6.30 & $\dagger$ & & & \\
\hline tEME-EA & $14_{6,9}-14_{5,10}$ & 263531.461 & 82.0 & 6.30 & $\ldots$ & $\ldots$ & 0.18 & $\mathrm{CH}_{3} \mathrm{OCH}_{3}$ \\
\hline tEME-EA & $14_{6,8}-14_{5,9}$ & 263531.520 & 82.0 & 6.30 & $\dddot{\dagger}$ & & & \\
\hline tEME-AA & $14_{6,8}-14_{5,9}$ & 263531.953 & 82.0 & 6.30 & $\dagger$ & & & \\
\hline tEME-AA & $14_{6,9}-14_{5,10}$ & 263531.971 & 82.0 & 6.30 & $\dagger$ & & & \\
\hline tEME-EE & $13_{6,7}-13_{5,8}$ & 263540.536 & 76.6 & 5.71 & $\ldots$ & $\ldots$ & 0.15 & $\mathrm{NH}_{2} \mathrm{CHO}, \mathrm{SO}_{2}$ \\
\hline tEME-EE' & $13_{6,7}-13_{5,8}$ & 263540.595 & 76.6 & 5.71 & $\dagger$ & & & \\
\hline tEME-AE & $13_{6,7}-13_{5,8}$ & 263541.040 & 76.6 & 5.71 & $\dagger$ & & & \\
\hline tEME-EE' & $13_{6,8}-13_{5,9}$ & 263541.858 & 76.6 & 5.71 & $\dagger$ & & & \\
\hline tEME-EE & $13_{6,8}-13_{5,9}$ & 263541.917 & 76.6 & 5.71 & $\dagger$ & & & \\
\hline tEME-AE & $13_{6,8}-13_{5,9}$ & 263542.362 & 76.6 & 5.71 & $\dagger$ & & & \\
\hline tEME-EA & $13_{6,8}-13_{5,9}$ & 263544.188 & 76.6 & 5.71 & $\ldots$ & $\ldots$ & 0.18 & $\mathrm{NH}_{2} \mathrm{CHO}, \mathrm{SO}_{2}$ \\
\hline tEME-EA & $13_{6,7}-13_{5,8}$ & 263544.247 & 76.6 & 5.71 & $\dddot{\dagger}$ & & & \\
\hline tEME-AA & $13_{6,7}-13_{5,8}$ & 263544.687 & 76.6 & 5.71 & $\dagger$ & & & \\
\hline tEME-AA & $13_{6,8}-13_{5,9}$ & 263544.696 & 76.6 & 5.71 & $\dagger$ & & & \\
\hline tEME-EE & $12_{6,6}-125,7$ & 263550.619 & 71.5 & 5.11 & $\ldots$ & $\ldots$ & 0.14 & $\mathrm{SO}_{2}$ \\
\hline tEME-EE' & $12_{6,6}-12_{5,7}$ & 263550.679 & 71.5 & 5.11 & $\dagger$ & & & \\
\hline tEME-AE & $12_{6,6}-125,7$ & 263551.125 & 71.5 & 5.11 & $\dagger$ & & & \\
\hline tEME-EE' & $12_{6,7}-12_{5,8}$ & 263551.945 & 71.5 & 5.11 & $\dagger$ & & & \\
\hline tEME-EE & $12_{6,7}-125,8$ & 263552.004 & 71.5 & 5.11 & $\dagger$ & & & \\
\hline tEME-AE & $12_{6,7}-12_{5,8}$ & 263552.451 & 71.5 & 5.11 & $\dagger$ & & & \\
\hline tEME-EA & $12_{6,7}-12_{5,8}$ & 263554.279 & 71.5 & 5.11 & $\ldots$ & $\ldots$ & 0.16 & $\mathrm{SO}_{2}$ \\
\hline tEME-EA & $12_{6,6}-12_{5,7}$ & 263554.338 & 71.5 & 5.11 & $\dagger$ & & & \\
\hline tEME-AA & $12_{6,6}-12_{5,7}$ & 263554.783 & 71.5 & 5.11 & $\dagger$ & & & \\
\hline tEME-AA & $12_{6,7}-125,8$ & 263554.787 & 71.5 & 5.11 & $\doteqdot$ & & & \\
\hline tEME-EE & $11_{6,5}-11_{5,6}$ & 263558.451 & 66.9 & 4.49 & $\ldots$ & $\ldots$ & 0.13 & $\mathrm{SO}_{2}$ \\
\hline tEME-EE' & $11_{6,5}-11_{5,6}$ & 263558.511 & 66.9 & 4.49 & $\dagger$ & & & \\
\hline tEME-AE & $11_{6,5}-11_{5,6}$ & 263558.959 & 66.9 & 4.49 & $\dagger$ & & & \\
\hline tEME-EE' & $11_{6,6}-11_{5,7}$ & 263559.780 & 66.9 & 4.49 & $\ldots$ & $\ldots$ & 0.13 & $\mathrm{SO}_{2}$ \\
\hline tEME-EE & $11_{6,6}-11_{5,7}$ & 263559.839 & 66.9 & 4.49 & $\dagger$ & & & \\
\hline tEME-AE & $11_{6,6}-11_{5,7}$ & 263560.288 & 66.9 & 4.49 & $\dagger$ & & & \\
\hline tEME-EA & $11_{6,6}-11_{5,7}$ & 263562.118 & 66.9 & 4.49 & $\ldots$ & $\ldots$ & 0.15 & $\mathrm{SO}_{2}$ \\
\hline tEME-EA & $11_{6,5}-11_{5,6}$ & 263562.178 & 66.9 & 4.49 & $\dagger$ & & & \\
\hline
\end{tabular}


A\&A 582, L1 (2015)

Table A.2. continued.

\begin{tabular}{|c|c|c|c|c|c|c|c|c|}
\hline Species & $\begin{array}{c}\text { Transition } \\
J_{K_{\mathrm{a}}, K_{\mathrm{c}}}-J_{K_{\mathrm{a}}^{\prime}, K_{\mathrm{c}}^{\prime}}^{\prime}\end{array}$ & $\begin{array}{c}\text { Predicted } \\
\text { frequency }(\mathrm{MHz})\end{array}$ & $\begin{array}{r}E_{\text {upp }} \\
(\mathrm{K})\end{array}$ & $S_{i j}$ & $\begin{array}{c}\text { Observed }^{1} \\
\text { frequency }(\mathrm{MHz})\end{array}$ & $\begin{array}{c}\text { Observed }^{1} \\
T_{\mathrm{MB}}(\mathrm{K})\end{array}$ & $\begin{array}{c}\text { Model }^{2} \\
T_{\mathrm{MB}}(\mathrm{K})\end{array}$ & Blends \\
\hline tEME-AA & $11_{6,5}-11_{5,6}$ & 263562.626 & 66.9 & 4.49 & $\bar{\dagger}$ & & & \\
\hline tEME-AA & $11_{6,6}-11_{5,7}$ & 263562.627 & 66.9 & 4.49 & $\dagger$ & & & \\
\hline tEME-EE & $10_{6,4}-10_{5,5}$ & 263564.386 & 62.7 & 3.85 & $\ldots$ & $\ldots$ & 0.11 & $\mathrm{SO}_{2}$ \\
\hline tEME-EE' & $10_{6,4}-10_{5,5}$ & 263564.446 & 62.7 & 3.85 & $\dagger$ & & & \\
\hline tEME-AE & $10_{6,4}-10_{5,5}$ & 263564.897 & 62.7 & 3.85 & $\dagger$ & & & \\
\hline tEME-EE' & $10_{6,5}-10_{5,6}$ & 263565.718 & 62.7 & 3.85 & $\ldots$ & $\ldots$ & 0.11 & $\mathrm{SO}_{2}$ \\
\hline tEME-EE & $10_{6,5}-10_{5,6}$ & 263565.777 & 62.7 & 3.85 & $\dagger$ & & & \\
\hline tEME-AE & $10_{6,5}-10_{5,6}$ & 263566.228 & 62.7 & 3.85 & $\dagger$ & & & \\
\hline tEME-EA & $10_{6,5}-10_{5,6}$ & 263568.061 & 62.7 & 3.85 & $\ldots$ & $\ldots$ & 0.21 & $\mathrm{SO}_{2}$ \\
\hline tEME-EA & $10_{6,4}-10_{5,5}$ & 263568.120 & 62.7 & 3.85 & $\dagger$ & & & \\
\hline tEME-AA & $10_{6,4}-10_{5,5}$ & 263568.570 & 62.7 & 3.85 & $\dagger$ & & & \\
\hline tEME-AA & $10_{6,5}-10_{5,6}$ & 263568.571 & 62.7 & 3.85 & $\dagger$ & & & \\
\hline tEME-EE & $9_{6,3}-9_{5,4}$ & 263568.750 & 58.8 & 3.19 & $\dagger$ & & & \\
\hline tEME-EE' & $9_{6,3}-9_{5,4}$ & 263568.810 & 58.8 & 3.19 & $\dagger$ & & & \\
\hline tEME-AE & $9_{6,3}-9_{5,4}$ & 263569.262 & 58.8 & 3.19 & $\dagger$ & & & \\
\hline tEME-EE' & $9_{6,4}-9_{5,5}$ & 263570.083 & 58.8 & 3.19 & $\dagger$ & & & \\
\hline tEME-EE & $9_{6,4}-9_{5,5}$ & 263570.143 & 58.8 & 3.19 & $\dagger$ & & & \\
\hline tEME-AE & $9_{6,4}-9_{5,5}$ & 263570.595 & 58.8 & 3.19 & $\dagger$ & & & \\
\hline tEME-AE & $8_{6,2}-8_{5,3}$ & 263572.350 & 55.3 & 2.50 & 263572.9 & 0.47 & 0.20 & $\mathrm{CH}_{3} \mathrm{OCH}_{3}, \mathrm{HNCO}$ \\
\hline tEME-EA & $9_{6,4}-9_{5,5}$ & 263572.430 & 58.8 & 3.19 & $\dagger$ & & & \\
\hline tEME-EA & $9_{6,3}-9_{5,4}$ & 263572.490 & 58.8 & 3.19 & $\dagger$ & & & \\
\hline tEME-AA & $9_{6,3}-9_{5,4}$ & 263572.942 & 58.8 & 3.19 & $\dagger$ & & & \\
\hline tEME-AA & $9_{6,4}-9_{5,5}$ & 263572.942 & 58.8 & 3.19 & $\dagger$ & & & \\
\hline tEME-EE' & $8_{6,3}-8_{5,4}$ & 263573.172 & 55.3 & 2.50 & $\dagger$ & & & \\
\hline tEME-EE & $8_{6,3}-8_{5,4}$ & 263573.232 & 55.3 & 2.50 & $\dagger$ & & & \\
\hline tEME-AE & $8_{6,3}-8_{5,4}$ & 263573.685 & 55.3 & 2.50 & $\dagger$ & & & \\
\hline tEME-EE & $7_{6,1}-7_{5,2}$ & 263573.911 & 52.2 & 1.75 & $\dagger$ & & & \\
\hline tEME-EE' & $7_{6,1}-7_{5,2}$ & 263573.972 & 52.2 & 1.75 & $\dagger$ & & & \\
\hline tEME-AE & $7_{6,1}-7_{5,2}$ & 263574.426 & 52.2 & 1.75 & $\dagger$ & & & \\
\hline tEME-EE & $6_{6,0}-6_{5,1}$ & 263575.211 & 49.5 & 0.93 & $\ldots$ & $\ldots$ & 0.17 & $\mathrm{CH}_{3} \mathrm{OCH}_{3}, \mathrm{HNCO}$ \\
\hline tEME-EE' & $7_{6,2}-7_{5,3}$ & 263575.249 & 52.2 & 1.75 & $\dagger$ & & & \\
\hline tEME-EE' & $6_{6,0}-6_{5,1}$ & 263575.271 & 49.5 & 0.93 & $\dagger$ & & & \\
\hline tEME-EE & $7_{6,2}-7_{5,3}$ & 263575.309 & 52.2 & 1.75 & $\dagger$ & & & \\
\hline tEME-EA & $8_{6,3}-8_{5,4}$ & 263575.522 & 55.3 & 2.50 & $\dagger$ & & & \\
\hline tEME-EA & $8_{6,2}-8_{5,3}$ & 263575.582 & 55.3 & 2.50 & $\dagger$ & & & \\
\hline tEME-AE & $6_{6,0}-6_{5,1}$ & 263575.727 & 49.5 & 0.93 & $\dagger$ & & & \\
\hline tEME-AE & $7_{6,2}-7_{5,3}$ & 263575.764 & 52.2 & 1.75 & $\dagger$ & & & \\
\hline tEME-AE & $6_{6,1}-6_{5,2}$ & 263577.066 & 49.5 & 0.93 & $\ldots$ & $\ldots$ & 0.08 & $\mathrm{CH}_{3} \mathrm{OCH}_{3}, \mathrm{HNCO}$ \\
\hline tEME-EA & $7_{6,2}-7_{5,3}$ & 263577.602 & 52.2 & 1.75 & $\dagger$ & & & \\
\hline tEME-EA & $7_{6,1}-7_{5,2}$ & 263577.662 & 52.2 & 1.75 & $\dagger$ & & & \\
\hline tEME-AA & $7_{6,1}-7_{5,2}$ & 263578.117 & 52.2 & 1.75 & 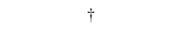 & & & \\
\hline tEME-AA & $7_{6,2}-7_{5,3}$ & 263578.117 & 52.2 & 1.75 & $\dagger$ & & & \\
\hline tEME-EA & $6_{6,1}-6_{5,2}$ & 263578.906 & 49.5 & 0.93 & $\dagger$ & & & \\
\hline tEME-EA & $6_{6,0}-6_{5,1}$ & 263578.966 & 49.5 & 0.93 & $\dagger$ & & & \\
\hline tEME-AA & $6_{6,0}-6_{5,1}$ & 263579.422 & 49.5 & 0.93 & $\dagger$ & & & \\
\hline tEME-AA & $6_{6,1}-6_{5,2}$ & 263579.422 & 49.5 & 0.93 & $\dagger$ & & & \\
\hline tEME-EE & $6_{5,1}-5_{4,1}$ & 263974.290 & 36.9 & 4.57 & 263974.6 & 0.84 & 0.32 & $\mathrm{CH}_{3} \mathrm{CH}_{2} \mathrm{CN} v_{20}=1, \mathrm{CH}_{3}{ }^{13} \mathrm{CH}_{2} \mathrm{CN}$ \\
\hline tEME-EE' & $6_{5,2}-5_{4,2}$ & 263974.352 & 36.9 & 4.57 & $\dagger$ & & & \\
\hline tEME-EE' & $6_{5,1}-5_{4,1}$ & 263974.491 & 36.9 & 4.57 & $\dagger$ & & & \\
\hline tEME-EE & $6_{5,2}-5_{4,2}$ & 263974.554 & 36.9 & 4.57 & $\dagger$ & & & \\
\hline tEME-AE & $6_{5,1}-5_{4,1}$ & 263974.847 & 36.9 & 4.57 & $\dagger$ & & & \\
\hline tEME-AE & $6_{5,2}-5_{4,2}$ & 263974.909 & 36.9 & 4.57 & $\dagger$ & & & \\
\hline tEME-EA & $6_{5,2}-5_{4,2}$ & 263977.560 & 36.9 & 4.57 & 263977.9 & 1.19 & 0.21 & $\mathrm{CH}_{3} \mathrm{CH}_{2} \mathrm{CN} v_{20}=1, \mathrm{CH}_{3}{ }^{13} \mathrm{CH}_{2} \mathrm{CN}, \mathrm{CH}_{3} \mathrm{OCOH} v_{t}=1$ \\
\hline tEME-EA & $6_{5,1}-5_{4,1}$ & 263977.761 & 36.9 & 4.57 & $\dagger$ & & & \\
\hline tEME-AA & $6_{5,2}-5_{4,1}$ & 263978.116 & 36.9 & 4.57 & $\dagger$ & & & \\
\hline tEME-AA & $6_{5,1}-5_{4,2}$ & 263978.117 & 36.9 & 4.57 & $\dagger$ & & & \\
\hline tEME-EE' & $12_{4,9}-11_{3,8}$ & 264279.974 & 48.6 & 1.86 & $\ldots$ & $\ldots$ & 0.04 & $\mathrm{CH}_{3} \mathrm{CH}_{2} \mathrm{CN} v_{13} / v_{21}$ \\
\hline tEME-AE & $12_{4,9}-11_{3,8}$ & 264281.470 & 48.6 & 1.98 & $\dagger$ & & & \\
\hline tEME-EE & $12_{4,8}-11_{3,8}$ & 264282.160 & 48.6 & 2.11 & 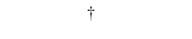 & & & \\
\hline tEME-EA & $12_{4,9}-11_{3,8}$ & 264288.936 & 48.6 & 3.18 & 264290.1 & 0.32 & 0.08 & $\mathrm{CH}_{3} \mathrm{CH}_{2} \mathrm{CN} v_{13} / v_{21}$ \\
\hline tEME-AA & $12_{4,9}-11_{3,8}$ & 264289.975 & 48.6 & 4.89 & $\dagger$ & & & \\
\hline tEME-EE & $12_{4,8}-11_{3,8}$ & 264290.781 & 48.6 & 2.78 & $\dagger$ & & & \\
\hline tEME-EA & $12_{4,8}-11_{3,8}$ & 264290.868 & 48.6 & 1.70 & $\dagger$ & & & \\
\hline tEME-AE & $12_{4,8}-11_{3,8}$ & 264291.901 & 48.6 & 2.91 & 264292.2 & 0.33 & 0.07 & $\mathrm{CH}_{3} \mathrm{CH}_{2} \mathrm{CN} v_{13} / v_{21}$ \\
\hline tEME-EE' & $12_{4,8}-11_{3,8}$ & 264292.217 & 48.6 & 3.02 & $\dagger$ & & & \\
\hline tEME-EE' & $12_{4,9}-11_{3,9}$ & 264316.948 & 48.6 & 3.02 & $\ldots$ & $\ldots$ & 0.06 & $\mathrm{CH}_{3} \mathrm{OH}$ \\
\hline tEME-AE & $12_{4,9}-11_{3,9}$ & 264318.041 & 48.6 & 2.91 & $\dagger$ & & & \\
\hline tEME-EE & $12_{4,9}-11_{3,9}$ & 264318.385 & 48.6 & 2.78 & $\dagger$ & & & \\
\hline tEME-EA & $12_{4,9}-11_{3,9}$ & 264324.363 & 48.6 & 1.70 & $\ldots$ & $\ldots$ & 0.10 & $\mathrm{CH}_{3} \mathrm{OH}$ \\
\hline tEME-AA & $12_{4,8}-11_{3,9}$ & 264326.032 & 48.6 & 4.89 & $\dagger$ & & & \\
\hline tEME-EA & $12_{4,8}-11_{3,9}$ & 264326.294 & 48.6 & 3.18 & $\dagger$ & & & \\
\hline tEME-EE & $12_{4,8}-11_{3,9}$ & 264327.005 & 48.6 & 2.11 & $\dagger$ & & & \\
\hline tEME-AE & $12_{4,8}-11_{3,9}$ & 264328.472 & 48.6 & 1.98 & $\ldots$ & $\ldots$ & 0.04 & $\mathrm{CH}_{3} \mathrm{OH}$ \\
\hline
\end{tabular}


B. Tercero et al.: Trans ethyl methyl ether in space

Table A.2. continued.

\begin{tabular}{|c|c|c|c|c|c|c|c|c|}
\hline Species & $\begin{array}{c}\text { Transition } \\
J_{K_{\mathrm{a}}, K_{\mathrm{c}}}-J_{K_{\mathrm{a}}^{\prime}, K_{\mathrm{c}}^{\prime}}^{\prime}\end{array}$ & $\begin{array}{c}\text { Predicted } \\
\text { frequency }(\mathrm{MHz})\end{array}$ & $\begin{array}{r}E_{\mathrm{upp}} \\
(\mathrm{K})\end{array}$ & $S_{i j}$ & $\begin{array}{c}\text { Observed }^{1} \\
\text { frequency }(\mathrm{MHz})\end{array}$ & $\begin{array}{c}\text { Observed }^{1} \\
T_{\mathrm{MB}}(\mathrm{K})\end{array}$ & $\begin{array}{c}\text { Model }^{2} \\
T_{\mathrm{MB}}(\mathrm{K})\end{array}$ & Blends \\
\hline tEME-EE' & $12_{4,8}-11_{3,9}$ & 264329.191 & 48.6 & 1.86 & $\dot{\dagger}$ & & & \\
\hline tEME-EE' & $30_{2,29}-29_{1,28}$ & 264623.050 & 183.6 & 14.10 & $\ldots$ & $\ldots$ & 0.14 & $\mathrm{CH}_{3} \mathrm{CH}_{2} \mathrm{CN} v_{13} / v_{21}$ \\
\hline tEME-EE & $30_{2,29}-29_{1,28}$ & 264623.050 & 183.6 & 14.10 & $\dagger$ & & & \\
\hline tEME-AE & $30_{2,29}-29_{1,28}$ & 264623.125 & 183.6 & 14.10 & $\dagger$ & & & \\
\hline tEME-EA & $30_{2,29}-29_{1,28}$ & 264624.097 & 183.6 & 14.10 & $\dagger$ & & & \\
\hline tEME-AA & $30_{2,29}-29_{1,28}$ & 264624.172 & 183.6 & 14.10 & $\dagger$ & & & \\
\hline tEME-AA & $35_{1,34}-34_{2,33}$ & 265627.614 & 246.2 & 18.59 & $\ldots$ & $\ldots$ & 0.13 & $\mathrm{CH}_{3} \mathrm{COOCH}_{3}$ \\
\hline tEME-EA & $35_{1,34}-34_{2,33}$ & 265627.630 & 246.2 & 18.59 & $\dagger$ & & & \\
\hline tEME-AE & $35_{1,34}-34_{2,33}$ & 265628.218 & 246.2 & 18.59 & $\dagger$ & & & \\
\hline tEME-EE & $35_{1,34}-34_{2,33}$ & 265628.234 & 246.2 & 18.59 & $\dagger$ & & & \\
\hline tEME-EE' & $35_{1,34}-34_{2,33}$ & 265628.234 & 246.2 & 18.59 & $\dagger$ & & & \\
\hline tEME-EE' & $19_{3,17}-18_{2,16}$ & 265859.733 & 83.8 & 5.59 & $\ldots$ & $\ldots$ & 0.13 & $\mathrm{HCN}$ \\
\hline tEME-EE & $19_{3,17}-18_{2,16}$ & 265859.748 & 83.8 & 5.59 & $\ddot{\dagger}$ & & & \\
\hline tEME-AE & $19_{3,17}-18_{2,16}$ & 265860.022 & 83.8 & 5.59 & $\dagger$ & & & \\
\hline tEME-EA & $19_{3,17}-18_{2,16}$ & 265862.261 & 83.8 & 5.59 & $\ldots$ & $\ldots$ & 0.09 & $\mathrm{HCN}$ \\
\hline tEME-AA & $19_{3,17}-18_{2,16}$ & 265862.543 & 83.8 & 5.59 & $\dagger$ & & & \\
\hline tEME-EA & $34_{0,34}-33_{1,33}$ & 266065.053 & 226.0 & 28.77 & $\ldots$ & $\ldots$ & 0.28 & $\mathrm{CH}_{3} \mathrm{COCH}_{3}$ \\
\hline tEME-AA & $34_{0,34}-33_{1,33}$ & 266065.056 & 226.0 & 28.77 & $\dddot{\dagger}$ & & & \\
\hline tEME-EE & $34_{0,34}-33_{1,33}$ & 266065.087 & 226.0 & 28.77 & $\dagger$ & & & \\
\hline tEME-EE' & $34_{0,34}-33_{1,33}$ & 266065.087 & 226.0 & 28.77 & $\dagger$ & & & \\
\hline tEME-AE & $34_{0,34}-33_{1,33}$ & 266065.091 & 226.0 & 28.77 & $\dagger$ & & & \\
\hline tEME-EE & $18_{3,15}-17_{2,16}$ & 267851.372 & 76.5 & 5.09 & 267851.6 & 0.71 & 0.13 & $\mathrm{CH}_{3} \mathrm{OCOH} v_{t}=2$ \\
\hline tEME-EE' & $18_{3,15}-17_{2,16}$ & 267851.394 & 76.5 & 5.09 & $\dagger$ & & & \\
\hline tEME-AE & $18_{3,15}-17_{2,16}$ & 267851.682 & 76.5 & 5.09 & $\dagger$ & & & \\
\hline tEME-EA & $18_{3,15}-17_{2,16}$ & 267853.764 & 76.5 & 5.09 & $\ldots$ & $\ldots$ & 0.09 & $\mathrm{CH}_{3} \mathrm{OCOH} v_{t}=2$ \\
\hline tEME-AA & $18_{3,15}-17_{2,16}$ & 267854.063 & 76.5 & 5.09 & $\dagger$ & & & \\
\hline tEME-EE' & $34_{1,34}-33_{0,33}$ & 267994.638 & 226.1 & 28.78 & 267994.7 & 0.95 & 0.28 & $\mathrm{CH}_{3} \mathrm{OCOH} v_{t}=2, \mathrm{CH}_{3} \mathrm{CH}_{2} \mathrm{CN}$ \\
\hline tEME-EE & $34_{1,34}-33_{0,33}$ & 267994.638 & 226.1 & 28.78 & $\dagger$ & & & \\
\hline tEME-AE & $34_{1,34}-33_{0,33}$ & 267994.646 & 226.1 & 28.78 & $\dagger$ & & & \\
\hline tEME-EA & $34_{1,34}-33_{0,33}$ & 267994.729 & 226.1 & 28.78 & $\dagger$ & & & \\
\hline tEME-AA & $34_{1,34}-33_{0,33}$ & 267994.737 & 226.1 & 28.78 & $\dagger$ & & & \\
\hline tEME-EE' & $31_{2,30}-30_{1,29}$ & 270379.929 & 195.5 & 14.99 & 270380.3 & 0.21 & 0.15 & \\
\hline tEME-EE & $31_{2,30}-30_{1,29}$ & 270379.929 & 195.5 & 14.99 & $\dagger$ & & & \\
\hline tEME-AE & $31_{2,30}-30_{1,29}$ & 270379.998 & 195.5 & 14.99 & $\dagger$ & & & \\
\hline tEME-EA & $31_{2,30}-30_{1,29}$ & 270380.924 & 195.5 & 14.99 & $\dagger$ & & & \\
\hline tEME-AA & $31_{2,30}-30_{1,29}$ & 270380.993 & 195.5 & 14.99 & $\dagger$ & & & \\
\hline tEME-EE & $7_{5,2}-6_{4,2}$ & 272025.103 & 39.6 & 4.70 & 272025.5 & 1.10 & 0.35 & $\mathrm{CH}_{3} \mathrm{CH}_{2} \mathrm{CN} v_{13} / v_{21}$ \\
\hline tEME-EE' & $7_{5,3}-6_{4,3}$ & 272025.167 & 39.6 & 4.70 & $\dot{\dagger}$ & & & \\
\hline tEME-EE' & $75,2-64,2$ & 272025.304 & 39.6 & 4.70 & $\dagger$ & & & \\
\hline tEME-EE & $7_{5,3}-6_{4,3}$ & 272025.368 & 39.6 & 4.70 & $\dagger$ & & & \\
\hline tEME-AE & $7_{5,2}-6_{4,2}$ & 272025.659 & 39.6 & 4.70 & $\dagger$ & & & \\
\hline tEME-AE & $7_{5,3}-6_{4,3}$ & 272025.723 & 39.6 & 4.70 & $\dagger$ & & & \\
\hline tEME-EA & $7_{5,3}-6_{4,3}$ & 272028.370 & 39.6 & 4.70 & 272028.6 & 0.59 & 0.23 & $\mathrm{CH}_{3} \mathrm{CH}_{2} \mathrm{CN} v_{13} / v_{21}$ \\
\hline tEME-EA & $7_{5,2}-6_{4,2}$ & 272028.571 & 39.6 & 4.70 & $\dagger$ & & & \\
\hline tEME-AA & $7_{5,3}-6_{4,2}$ & 272028.925 & 39.6 & 4.70 & $\dagger$ & & & \\
\hline tEME-AA & $7_{5,2}-6_{4,3}$ & 272028.927 & 39.6 & 4.70 & $\dagger$ & & & \\
\hline tEME-EE' & $13_{4,10}-12_{3,9}$ & 272291.842 & 53.6 & 2.35 & 272292.1 & 0.21 & 0.03 & U-line \\
\hline tEME-AE & $13_{4,10}-12_{3,9}$ & 272293.250 & 53.6 & 2.46 & 272293.6 & 0.26 & 0.05 & U-line \\
\hline tEME-EE & $13_{4,10}-12_{3,9}$ & 272293.863 & 53.6 & 2.58 & $\dagger$ & & & \\
\hline tEME-EA & $13_{4,10}-12_{3,9}$ & 272300.368 & 53.6 & 3.94 & 272301.0 & 0.18 & 0.08 & $\mathrm{CH}_{3} \mathrm{OCOH}$ \\
\hline tEME-AA & $13_{4,10}-12_{3,9}$ & 272301.238 & 53.6 & 5.09 & $\dagger$ & & & \\
\hline tEME-EE & $13_{4,9}-12_{3,9}$ & 272302.533 & 53.6 & 2.51 & $\dagger$ & & & \\
\hline tEME-EA & $13_{4,9}-12_{3,9}$ & 272302.564 & 53.6 & 1.15 & $\dagger$ & & & \\
\hline tEME-AE & $13_{4,9}-12_{3,9}$ & 272303.710 & 53.6 & 2.64 & 272303.9 & 0.24 & 0.07 & $\mathrm{CH}_{3} \mathrm{CH}_{2} \mathrm{CN} v_{20}=1$ \\
\hline tEME-EE' & $13_{4,9}-12_{3,9}$ & 272304.098 & 53.6 & 2.74 & $\dagger$ & & & \\
\hline tEME-EE' & $13_{4,10}-12_{3,10}$ & 272351.722 & 53.6 & 2.74 & $\ldots$ & $\ldots$ & 0.06 & $\mathrm{CH}_{3} \mathrm{OH}, \mathrm{CH}_{3} \mathrm{OD}$ \\
\hline tEME-AE & $13_{4,10}-12_{3,10}$ & 272352.884 & 53.6 & 2.63 & $\dddot{\dagger}$ & & & \\
\hline tEME-EE & $13_{4,10}-12_{3,10}$ & 272353.288 & 53.6 & 2.51 & $\dagger$ & & & \\
\hline tEME-EA & $13_{4,10}-12_{3,10}$ & 272359.310 & 53.6 & 1.15 & $\ldots$ & $\ldots$ & 0.13 & $\mathrm{CH}_{3} \mathrm{OH}, \mathrm{CH}_{3} \mathrm{OD}$ \\
\hline tEME-AA & $13_{4,9}-12_{3,10}$ & 272361.410 & 53.6 & 5.09 & $\dddot{\dagger}$ & & & \\
\hline tEME-EA & $13_{4,9}-12_{3,10}$ & 272361.506 & 53.6 & 3.94 & $\dagger$ & & & \\
\hline tEME-EE & $13_{4,9}-12_{3,10}$ & 272361.957 & 53.6 & 2.58 & $\dagger$ & & & \\
\hline tEME-AE & $13_{4,9}-12_{3,10}$ & 272363.344 & 53.6 & 2.46 & $\ldots$ & $\ldots$ & 0.05 & $\mathrm{CH}_{3} \mathrm{OH}, \mathrm{CH}_{3} \mathrm{OD}$ \\
\hline tEME-EE' & $13_{4,9}-12_{3,10}$ & 272363.978 & 53.6 & 2.35 & $\dddot{\dagger}$ & & & \\
\hline tEME-EE' & $20_{3,18}-19_{2,17}$ & 272432.754 & 91.6 & 5.79 & 272433.0 & 0.33 & 0.14 & U-line \\
\hline tEME-EE & $20_{3,18}-19_{2,17}$ & 272432.765 & 91.6 & 5.79 & $\dagger$ & & & \\
\hline tEME-AE & $20_{3,18}-19_{2,17}$ & 272433.037 & 91.6 & 5.79 & $\dagger$ & & & \\
\hline tEME-EA & $20_{3,18}-19_{2,17}$ & 272435.273 & 91.6 & 5.79 & 272435.7 & 0.35 & 0.09 & U-line \\
\hline tEME-AA & $20_{3,18}-19_{2,17}$ & 272435.550 & 91.6 & 5.79 & $\dot{\dagger}$ & & & \\
\hline tEME-AA & $40_{2,38}-39_{3,37}$ & 272596.983 & 325.0 & 12.66 & $\ldots$ & $\ldots$ & 0.03 & U-line \\
\hline tEME-EA & $40_{2,38}-39_{3,37}$ & 272597.055 & 325.0 & 12.66 & $\dddot{\dagger}$ & & & \\
\hline tEME-AE & $40_{2,38}-39_{3,37}$ & 272598.688 & 325.0 & 12.66 & $\dagger$ & & & \\
\hline tEME-EE & $40_{2,38}-39_{3,37}$ & 272598.760 & 325.0 & 12.66 & $\dagger$ & & & \\
\hline
\end{tabular}


A\&A 582, L1 (2015)

Table A.2. continued.

\begin{tabular}{|c|c|c|c|c|c|c|c|c|}
\hline Species & $\begin{array}{c}\text { Transition } \\
J_{K_{\mathrm{a}}, K_{\mathrm{c}}}-J_{K_{\mathrm{a}}^{\prime}, K_{\mathrm{c}}^{\prime}}^{\prime}\end{array}$ & $\begin{array}{c}\text { Predicted } \\
\text { frequency }(\mathrm{MHz})\end{array}$ & $\begin{array}{r}E_{\mathrm{upp}} \\
(\mathrm{K})\end{array}$ & $S_{i j}$ & $\begin{array}{c}\text { Observed }^{1} \\
\text { frequency }(\mathrm{MHz})\end{array}$ & $\begin{array}{c}\text { Observed }^{1} \\
T_{\mathrm{MB}}(\mathrm{K})\end{array}$ & $\begin{array}{c}\text { Model }^{2} \\
T_{\mathrm{MB}}(\mathrm{K})\end{array}$ & Blends \\
\hline tEME-EE' & $40_{2,38}-39_{3,37}$ & 272598.760 & 325.0 & 12.66 & $\bar{\dagger}$ & & & \\
\hline tEME-EE & $20_{2,18}-19_{1,19}$ & 273141.137 & 86.3 & 2.00 & $\ldots$ & $\ldots$ & 0.05 & $\mathrm{CH}_{3} \mathrm{OCOH}$ \\
\hline tEME-EE' & $20_{2,18}-19_{1,19}$ & 273141.138 & 86.3 & 2.00 & $\dagger$ & & & \\
\hline tEME-AE & $20_{2,18}-19_{1,19}$ & 273141.411 & 86.3 & 2.00 & $\dagger$ & & & \\
\hline tEME-EA & $20_{2,18}-19_{1,19}$ & 273142.818 & 86.3 & 2.00 & $\ldots$ & $\ldots$ & 0.03 & $\mathrm{CH}_{3} \mathrm{OCOH}$ \\
\hline tEME-AA & $20_{2,18}-19_{1,19}$ & 273143.092 & 86.3 & 2.00 & $\dagger$ & & & \\
\hline tEME-EA & $35_{0,35}-34_{1,34}$ & 273979.063 & 239.2 & 29.79 & 273979.1 & 0.73 & 0.28 & $\mathrm{CH}_{3} \mathrm{CH}_{2} \mathrm{CN}, \mathrm{SO}_{2}$ \\
\hline tEME-AA & $35_{0,35}-34_{1,34}$ & 273979.067 & 239.2 & 29.79 & $\dagger$ & & & \\
\hline tEME-EE & $35_{0,35}-34_{1,34}$ & 273979.089 & 239.2 & 29.79 & $\dagger$ & & & \\
\hline tEME-EE' & $35_{0,35}-34_{1,34}$ & 273979.089 & 239.2 & 29.79 & 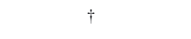 & & & \\
\hline tEME-AE & $35_{0,35}-34_{1,34}$ & 273979.093 & 239.2 & 29.79 & $\dagger$ & & & \\
\hline tEME-AA & $36_{1,35}-35_{2,34}$ & 274946.543 & 260.0 & 19.65 & 274946.9 & 0.57 & 0.14 & $\mathrm{CH}_{3} \mathrm{OCOH} v_{t}=1, \mathrm{H}_{2} \mathrm{CS}$ \\
\hline tEME-EA & $36_{1,35}-35_{2,34}$ & 274946.555 & 260.0 & 19.65 & $\dagger$ & & & \\
\hline tEME-AE & $36_{1,35}-35_{2,34}$ & 274947.090 & 260.0 & 19.65 & $\dagger$ & & & \\
\hline tEME-EE & $36_{1,35}-35_{2,34}$ & 274947.102 & 260.0 & 19.65 & $\dagger$ & & & \\
\hline tEME-EE' & $36_{1,35}-35_{2,34}$ & 274947.102 & 260.0 & 19.65 & $\dagger$ & & & \\
\hline tEME-EE' & $35_{1,35}-34_{0,34}$ & 275618.927 & 239.3 & 29.80 & 275618.9 & 0.22 & 0.28 & \\
\hline tEME-EE & $35_{1,35}-34_{0,34}$ & 275618.927 & 239.3 & 29.80 & $\dagger$ & & & \\
\hline tEME-AE & $35_{1,35}-34_{0,34}$ & 275618.934 & 239.3 & 29.80 & $\dagger$ & & & \\
\hline tEME-EA & $35_{1,35}-34_{0,34}$ & 275619.009 & 239.3 & 29.80 & $\dagger$ & & & \\
\hline tEME-AA & $35_{1,35}-34_{0,34}$ & 275619.016 & 239.3 & 29.80 & $\dagger$ & & & \\
\hline tEME-EE' & $32_{2,31}-31_{1,30}$ & 276219.631 & 207.8 & 15.92 & 276219.6 & 0.34 & 0.15 & ${ }^{13} \mathrm{CH}_{3} \mathrm{OCOH}$ \\
\hline tEME-EE & $32_{2,31}-31_{1,30}$ & 276219.631 & 207.8 & 15.92 & $\dagger$ & & & \\
\hline tEME-AE & $32_{2,31}-31_{1,30}$ & 276219.695 & 207.8 & 15.92 & $\dagger$ & & & \\
\hline tEME-EA & $32_{2,31}-31_{1,30}$ & 276220.573 & 207.8 & 15.92 & $\dagger$ & & & \\
\hline tEME-AA & $32_{2,31}-31_{1,30}$ & 276220.637 & 207.8 & 15.92 & $\dagger$ & & & \\
\hline tEME-EE & $19_{3,16}-18_{2,17}$ & 276700.152 & 83.9 & 5.20 & 276700.3 & 0.29 & 0.13 & $\mathrm{CH}_{3} \mathrm{CN} v_{8}=1, \mathrm{CH}_{3} \mathrm{OH}$ \\
\hline tEME-EE' & $19_{3,16}-18_{2,17}$ & 276700.167 & 83.9 & 5.20 & $\dot{\dagger}$ & & & \\
\hline tEME-AE & $19_{3,16}-18_{2,17}$ & 276700.458 & 83.9 & 5.20 & $\dagger$ & & & \\
\hline tEME-EA & $19_{3,16}-18_{2,17}$ & 276702.533 & 83.9 & 5.20 & 276702.5 & 0.34 & 0.08 & $\mathrm{CH}_{3} \mathrm{CN} v_{8}=1, \mathrm{CH}_{3} \mathrm{OH}$ \\
\hline tEME-AA & $19_{3,16}-18_{2,17}$ & 276702.832 & 83.9 & 5.20 & $\dagger$ & & & \\
\hline tEME-EE' & $21_{3,19}-20_{2,18}$ & 278825.580 & 99.7 & 6.00 & 278825.7 & 0.39 & 0.14 & $\mathrm{CH}_{3} \mathrm{CH}_{2} \mathrm{CN}, \mathrm{CH}_{3} \mathrm{CH}_{2} \mathrm{CN} v_{20}=1, \mathrm{CH}_{3}{ }^{13} \mathrm{CH}_{2} \mathrm{CN}$ \\
\hline tEME-EE & $21_{3,19}-20_{2,18}$ & 278825.588 & 99.7 & 6.00 & $\dagger$ & & & \\
\hline tEME-AE & $21_{3,19}-20_{2,18}$ & 278825.856 & 99.7 & 6.00 & $\dagger$ & & & \\
\hline tEME-EA & $21_{3,19}-20_{2,18}$ & 278828.090 & 99.7 & 6.00 & 278828.4 & 0.33 & 0.09 & $\mathrm{CH}_{3} \mathrm{CH}_{2} \mathrm{CN}, \mathrm{CH}_{3} \mathrm{CH}_{2} \mathrm{CN} v_{20}=1, \mathrm{CH}_{3}{ }^{13} \mathrm{CH}_{2} \mathrm{CN}$ \\
\hline tEME-AA & $21_{3,19}-20_{2,18}$ & 278828.362 & 99.7 & 6.00 & $\dagger$ & & & \\
\hline tEME-EE & $8_{5,3}-7_{4,3}$ & 280075.262 & 42.7 & 4.85 & 280075.6 & 0.40 & 0.38 & \\
\hline tEME-EE' & $8_{5,4}-7_{4,4}$ & 280075.328 & 42.7 & 4.85 & $\dagger$ & & & \\
\hline tEME-EE' & $85,3-74,3$ & 280075.462 & 42.7 & 4.85 & $\dagger$ & & & \\
\hline tEME-EE & $8_{5,4}-7_{4,4}$ & 280075.527 & 42.7 & 4.85 & $\dagger$ & & & \\
\hline tEME-AE & $85,3-74,3$ & 280075.816 & 42.7 & 4.85 & $\dagger$ & & & \\
\hline tEME-AE & $8_{5,4}-7_{4,4}$ & 280075.882 & 42.7 & 4.85 & $\dagger$ & & & \\
\hline tEME-EA & $8_{5,4}-74,4$ & 280078.526 & 42.7 & 4.85 & 280078.5 & 0.31 & 0.25 & \\
\hline tEME-EA & $8_{5,3}-7_{4,3}$ & 280078.726 & 42.7 & 4.85 & $\dagger$ & & & \\
\hline tEME-AA & $8_{5,4}-7_{4,3}$ & 280079.076 & 42.7 & 4.85 & $\doteqdot$ & & & \\
\hline tEME-AA & $8_{5,3}-7_{4,4}$ & 280079.084 & 42.7 & 4.85 & $\dagger$ & & & \\
\hline tEME-EE' & $14_{4,11}-13_{3,10}$ & 280288.955 & 59.0 & 2.84 & $\ldots$ & $\ldots$ & 0.07 & $\mathrm{CH}_{3} \mathrm{COOH} v_{t}=1$ \\
\hline tEME-AE & $14_{4,11}-13_{3,10}$ & 280290.299 & 59.0 & 2.96 & $\dagger$ & & & \\
\hline tEME-EE & $14_{4,11}-13_{3,10}$ & 280290.853 & 59.0 & 3.11 & $\dagger$ & & & \\
\hline tEME-EA & $14_{4,11}-13_{3,10}$ & 280297.013 & 59.0 & 4.69 & 280297.7 & 0.12 & 0.10 & \\
\hline tEME-AA & $14_{4,11}-13_{3,10}$ & 280297.720 & 59.0 & 5.30 & $\dagger$ & & & \\
\hline tEME-EE & $14_{4,10}-13_{3,10}$ & 280299.704 & 59.0 & 2.19 & 280301.1 & 0.10 & 0.06 & U-line \\
\hline tEME-EA & $14_{4,10}-13_{3,10}$ & 280299.889 & 59.0 & 0.61 & $\dagger$ & & & \\
\hline tEME-AE & $14_{4,10}-13_{3,10}$ & 280300.898 & 59.0 & 2.34 & $\dagger$ & & & \\
\hline tEME-EE' & $14_{4,10}-13_{3,10}$ & 280301.316 & 59.0 & 2.46 & $\dagger$ & & & \\
\hline tEME-EE' & $14_{4,11}-13_{3,11}$ & 280383.694 & 59.0 & 2.46 & 280385.0 & 0.11 & 0.06 & $\mathrm{CH}_{3} \mathrm{OCOH} v_{t}=1$ \\
\hline tEME-AE & $14_{4,11}-13_{3,11}$ & 280384.884 & 59.0 & 2.34 & $\bar{\dagger}$ & & & \\
\hline tEME-EE & $14_{4,11}-13_{3,11}$ & 280385.307 & 59.0 & 2.19 & $\dagger$ & & & \\
\hline tEME-EA & $14_{4,10}-13_{3,11}$ & 280394.040 & 59.0 & 4.69 & $\ldots$ & $\ldots$ & 0.16 & $\mathrm{CH}_{3} \mathrm{OCOH}$ \\
\hline tEME-AA & $14_{4,10}-13_{3,11}$ & 280394.104 & 59.0 & 5.30 & $\dagger$ & & & \\
\hline tEME-EE & $14_{4,10}-13_{3,11}$ & 280394.157 & 59.0 & 3.11 & $\dagger$ & & & \\
\hline tEME-AE & $14_{4,10}-13_{3,11}$ & 280395.482 & 59.0 & 2.96 & $\dagger$ & & & \\
\hline tEME-EE' & $14_{4,10}-13_{3,11}$ & 280396.055 & 59.0 & 2.83 & $\dagger$ & & & \\
\hline tEME-EA & $36_{0,36}-35_{1,35}$ & 281871.802 & 252.8 & 30.81 & 281871.7 & 0.25 & 0.27 & U-line \\
\hline tEME-AA & $36_{0,36}-35_{1,35}$ & 281871.806 & 252.8 & 30.81 & $\dagger$ & & & \\
\hline tEME-EE & $36_{0,36}-35_{1,35}$ & 281871.822 & 252.8 & 30.81 & $\dagger$ & & & \\
\hline tEME-EE' & $36_{0,36}-35_{1,35}$ & 281871.822 & 252.8 & 30.81 & $\dagger$ & & & \\
\hline tEME-AE & $36_{0,36}-35_{1,35}$ & 281871.826 & 252.8 & 30.81 & $\dagger$ & & & \\
\hline tEME-EE' & $33_{2,32}-32_{1,31}$ & 282152.573 & 220.4 & 16.88 & 282153.2 & 0.56 & 0.16 & $\mathrm{HC}_{3} \mathrm{~N} v_{5}=1$ \\
\hline tEME-EE & $33_{2,32}-32_{1,31}$ & 282152.573 & 220.4 & 16.88 & $\dagger$ & & & \\
\hline tEME-AE & $33_{2,32}-32_{1,31}$ & 282152.631 & 220.4 & 16.88 & $\dagger$ & & & \\
\hline tEME-EA & $33_{2,32}-32_{1,31}$ & 282153.461 & 220.4 & 16.88 & $\dagger$ & & & \\
\hline tEME-AA & $33_{2,32}-32_{1,31}$ & 282153.519 & 220.4 & 16.88 & $\dagger$ & & & \\
\hline
\end{tabular}


B. Tercero et al.: Trans ethyl methyl ether in space

Table A.2. continued.

\begin{tabular}{|c|c|c|c|c|c|c|c|c|}
\hline Species & $\begin{array}{c}\text { Transition } \\
J_{K_{\mathrm{a}}, K_{\mathrm{c}}}-J_{K_{\mathrm{a}}^{\prime}, K_{\mathrm{c}}^{\prime}}^{\prime}\end{array}$ & $\begin{array}{c}\text { Predicted } \\
\text { frequency }(\mathrm{MHz})\end{array}$ & $\begin{array}{r}E_{\text {upp }} \\
(\mathrm{K})\end{array}$ & $S_{i j}$ & $\begin{array}{c}\text { Observed }^{1} \\
\text { frequency }(\mathrm{MHz})\end{array}$ & $\begin{array}{c}\text { Observed }^{1} \\
T_{\mathrm{MB}}(\mathrm{K})\end{array}$ & $\begin{array}{c}\text { Model }^{2} \\
T_{\mathrm{MB}}(\mathrm{K})\end{array}$ & Blends \\
\hline tEME-EE' & $36_{1,36}-35_{0,35}$ & 283263.283 & 252.8 & 30.82 & 283263.4 & 0.44 & 0.27 & ${ }^{13} \mathrm{CH}_{3} \mathrm{OH}$ \\
\hline tEME-EE & $36_{1,36}-35_{0,35}$ & 283263.283 & 252.8 & 30.82 & $\dagger$ & & & \\
\hline tEME-AE & $36_{1,36}-35_{0,35}$ & 283263.290 & 252.8 & 30.82 & $\dagger$ & & & \\
\hline tEME-EA & $36_{1,36}-35_{0,35}$ & 283263.357 & 252.8 & 30.82 & $\dagger$ & & & \\
\hline tEME-AA & $36_{1,36}-35_{0,35}$ & 283263.364 & 252.8 & 30.82 & $\dagger$ & & & \\
\hline tEME-AE & $41_{2,39}-40_{3,38}$ & 283951.422 & 341.0 & 13.47 & $\ldots$ & $\ldots$ & 0.03 & $\mathrm{CH}_{3} \mathrm{OCH}_{3}$ \\
\hline tEME-EE & $41_{2,39}-40_{3,38}$ & 283951.485 & 341.0 & 13.47 & $\dagger$ & & & \\
\hline tEME-EE' & $41_{2,39}-40_{3,38}$ & 283951.485 & 341.0 & 13.47 & $\dagger$ & & & \\
\hline tEME-AA & $37_{1,36}-36_{2,35}$ & 284137.669 & 274.2 & 20.72 & 288138.3 & 0.34 & 0.14 & $\mathrm{CH}_{3} \mathrm{OCOH} v_{t}=1$ \\
\hline tEME-EA & $37_{1,36}-36_{2,35}$ & 284137.677 & 274.2 & 20.72 & $\dagger$ & & & \\
\hline tEME-AE & $37_{1,36}-36_{2,35}$ & 284138.161 & 274.2 & 20.72 & $\dagger$ & & & \\
\hline tEME-EE & $37_{1,36}-36_{2,35}$ & 284138.169 & 274.2 & 20.72 & $\dot{\dagger}$ & & & \\
\hline tEME-EE' & $37_{1,36}-36_{2,35}$ & 284138.169 & 274.2 & 20.72 & $\dagger$ & & & \\
\hline tEME-EE' & $22_{3,20}-21_{2,19}$ & 285037.438 & 108.2 & 6.21 & $\ldots$ & $\ldots$ & 0.14 & $\mathrm{CH}_{3} \mathrm{CH}_{2} \mathrm{CN} v_{13} / v_{21}$ \\
\hline tEME-EE & $22_{3,20}-21_{2,19}$ & 285037.444 & 108.2 & 6.21 & $\dagger$ & & & \\
\hline tEME-AE & $22_{3,20}-21_{2,19}$ & 285037.708 & 108.2 & 6.21 & $\dagger$ & & & \\
\hline tEME-EA & $22_{3,20}-21_{2,19}$ & 285039.939 & 108.2 & 6.21 & $\ldots$ & $\ldots$ & 0.09 & $\mathrm{CH}_{3} \mathrm{CH}_{2} \mathrm{CN} v_{13} / v_{21}$ \\
\hline tEME-AA & $22_{3,20}-21_{2,19}$ & 285040.206 & 108.2 & 6.21 & $\dagger$ & & & \\
\hline tEME-EE & $20_{3,17}-19_{2,18}$ & 285699.473 & 91.6 & 5.28 & $\ldots$ & $\ldots$ & 0.14 & $\mathrm{SO}_{2}$ \\
\hline tEME-EE' & $20_{3,17}-19_{2,18}$ & 285699.484 & 91.6 & 5.28 & $\dddot{\dagger}$ & & & \\
\hline tEME-AE & $20_{3,17}-19_{2,18}$ & 285699.777 & 91.6 & 5.28 & $\dagger$ & & & \\
\hline tEME-EA & $20_{3,17}-19_{2,18}$ & 285701.840 & 91.6 & 5.28 & $\ldots$ & $\ldots$ & 0.10 & $\mathrm{SO}_{2}$ \\
\hline tEME-AA & $20_{3,17}-19_{2,18}$ & 285702.139 & 91.6 & 5.28 & $\dddot{\dagger}$ & & & \\
\hline tEME-EE & $21_{2,19}-20_{1,20}$ & 286585.825 & 94.5 & 1.86 & $\ldots$ & $\ldots$ & 0.05 & $\mathrm{CH}_{3} \mathrm{COOH} v_{t}=1$ \\
\hline tEME-EE' & $21_{2,19}-20_{1,20}$ & 286585.825 & 94.5 & 1.86 & $\dagger$ & & & \\
\hline tEME-AE & $21_{2,19}-20_{1,20}$ & 286586.110 & 94.5 & 1.86 & $\dagger$ & & & \\
\hline tEME-EA & $21_{2,19}-20_{1,20}$ & 286587.511 & 94.5 & 1.86 & $\ldots$ & $\ldots$ & 0.03 & U-line \\
\hline tEME-AA & $21_{2,19}-20_{1,20}$ & 286587.796 & 94.5 & 1.86 & $\dot{\dagger}$ & & & \\
\hline tEME-EE & $9_{5,4}-8_{4,4}$ & 288124.443 & 46.1 & 5.02 & 288124.8 & 0.59 & 0.41 & \\
\hline tEME-EE' & $9_{5,5}-8_{4,5}$ & 288124.510 & 46.1 & 5.02 & $\dagger$ & & & \\
\hline tEME-EE' & $9_{5,4}-8_{4,4}$ & 288124.641 & 46.1 & 5.02 & $\dagger$ & & & \\
\hline tEME-EE & $9_{5,5}-84,5$ & 288124.708 & 46.1 & 5.02 & $\doteqdot$ & & & \\
\hline tEME-AE & $9_{5,4}-8_{4,4}$ & 288124.995 & 46.1 & 5.02 & $\dagger$ & & & \\
\hline tEME-AE & $9,5-84,5$ & 288125.062 & 46.1 & 5.02 & $\dagger$ & & & \\
\hline tEME-EA & $9_{5,5}-8_{4,5}$ & 288127.703 & 46.1 & 5.02 & $\ldots$ & $\ldots$ & 0.27 & $\mathrm{CH}_{3} \mathrm{OCOH}$ \\
\hline tEME-EA & $9_{5,4}-8_{4,4}$ & 288127.901 & 46.1 & 5.02 & $\dagger$ & & & \\
\hline tEME-AA & $9_{5,5}-8_{4,4}$ & 288128.242 & 46.1 & 5.02 & $\dagger$ & & & \\
\hline tEME-AA & $9_{5,4}-8_{4,5}$ & 288128.268 & 46.1 & 5.02 & $\dagger$ & & & \\
\hline tEME-EE' & $34_{2,33}-33_{1,32}$ & 288187.160 & 233.4 & 17.86 & 288187.5 & 0.54 & 0.17 & $\mathrm{CH}_{3} \mathrm{OCOH}$ \\
\hline tEME-EE & $34_{2,33}-33_{1,32}$ & 288187.160 & 233.4 & 17.86 & $\dagger$ & & & \\
\hline tEME-AE & $34_{2,33}-33_{1,32}$ & 288187.214 & 233.4 & 17.86 & $\dagger$ & & & \\
\hline tEME-EA & $34_{2,33}-33_{1,32}$ & 288187.993 & 233.4 & 17.86 & $\dagger$ & & & \\
\hline tEME-AA & $34_{2,33}-33_{1,32}$ & 288188.047 & 233.4 & 17.86 & $\dagger$ & & & \\
\hline tEME-EE' & $15_{4,12}-14_{3,11}$ & 288267.539 & 64.8 & 3.40 & $\ldots$ & $\ldots$ & 0.09 & $\mathrm{SO}^{18} \mathrm{O}, \mathrm{CH}_{3} \mathrm{COCH}_{3}$ \\
\hline tEME-AE & $15_{4,12}-14_{3,11}$ & 288268.817 & 64.8 & 3.55 & $\dddot{\dagger}$ & & & \\
\hline tEME-EE & $15_{4,12}-14_{3,11}$ & 288269.302 & 64.8 & 3.75 & $\dot{\dagger}$ & & & \\
\hline tEME-EA & $15_{4,12}-14_{3,11}$ & 288274.971 & 64.8 & 5.24 & 288275.12 & 0.14 & 0.12 & $\mathrm{SO}^{18} \mathrm{O}$ \\
\hline tEME-AA & $15_{4,12}-14_{3,11}$ & 288275.555 & 64.8 & 5.50 & $\dagger$ & & & \\
\hline tEME-EE & $15_{4,11}-14_{3,11}$ & 288278.681 & 64.8 & 1.75 & $\ldots$ & $\ldots$ & 0.05 & U-line \\
\hline tEME-AE & $15_{4,11}-14_{3,11}$ & 288279.849 & 64.8 & 1.95 & $\dddot{\dagger}$ & & & \\
\hline tEME-EE' & $15_{4,11}-14_{3,11}$ & 288280.260 & 64.8 & 2.11 & $\dagger$ & & & \\
\hline tEME-EE' & $15_{4,12}-14_{3,12}$ & 288413.150 & 64.8 & 2.11 & $\ldots$ & $\ldots$ & 0.05 & U-line \\
\hline tEME-AE & $15_{4,12}-14_{3,12}$ & 288414.329 & 64.8 & 1.95 & $\dddot{\dagger}$ & & & \\
\hline tEME-EE & $15_{4,12}-14_{3,12}$ & 288414.729 & 64.8 & 1.75 & $\dagger$ & & & \\
\hline tEME-EE & $15_{4,11}-14_{3,12}$ & 288424.108 & 64.8 & 3.75 & 288424.7 & 0.50 & 0.20 & $\mathrm{CH}_{3} \mathrm{OC}^{18} \mathrm{OH}$ \\
\hline tEME-EA & $15_{4,11}-14_{3,12}$ & 288424.469 & 64.8 & 5.24 & $\dagger$ & & & \\
\hline tEME-AA & $15_{4,11}-14_{3,12}$ & 288424.653 & 64.8 & 5.50 & $\dagger$ & & & \\
\hline tEME-AE & $15_{4,11}-14_{3,12}$ & 288425.361 & 64.8 & 3.55 & $\dagger$ & & & \\
\hline tEME-EE' & $15_{4,11}-14_{3,12}$ & 288425.871 & 64.8 & 3.40 & $\dagger$ & & & \\
\hline tEME-EA & $37_{0,37}-36_{1,36}$ & 289745.973 & 266.7 & 31.83 & 289745.8 & 0.49 & 0.26 & U-line \\
\hline tEME-AA & $37_{0,37}-36_{1,36}$ & 289745.977 & 266.7 & 31.83 & $\dagger$ & & & \\
\hline tEME-EE & $37_{0,37}-36_{1,36}$ & 289745.986 & 266.7 & 31.83 & $\dagger$ & & & \\
\hline tEME-EE' & $37_{0,37}-36_{1,36}$ & 289745.986 & 266.7 & 31.83 & $\dagger$ & & & \\
\hline tEME-AE & $37_{0,37}-36_{1,36}$ & 289745.991 & 266.7 & 31.83 & $\dagger$ & & & \\
\hline tEME-EE' & $37_{1,37}-36_{0,36}$ & 290924.982 & 266.8 & 31.83 & 290925.0 & 0.71 & 0.27 & ${ }^{33} \mathrm{SO}_{2}$ \\
\hline tEME-EE & $37_{1,37}-36_{0,36}$ & 290924.982 & 266.8 & 31.83 & $\dagger$ & & & \\
\hline tEME-AE & $37_{1,37}-36_{0,36}$ & 290924.989 & 266.8 & 31.83 & $\dagger$ & & & \\
\hline tEME-EA & $37_{1,37}-36_{0,36}$ & 290925.049 & 266.8 & 31.83 & $\dagger$ & & & \\
\hline tEME-AA & $37_{1,37}-36_{0,36}$ & 290925.056 & 266.8 & 31.83 & $\dagger$ & & & \\
\hline tEME-AA & $38_{1,37}-37_{2,36}$ & 293203.620 & 288.8 & 21.79 & 293203.7 & 0.28 & 0.14 & \\
\hline tEME-EA & $38_{1,37}-37_{2,36}$ & 293203.624 & 288.8 & 21.79 & $\doteqdot$ & & & \\
\hline tEME-AE & $38_{1,37}-37_{2,36}$ & 293204.059 & 288.8 & 21.79 & $\dagger$ & & & \\
\hline tEME-EE & $38_{1,37}-37_{2,36}$ & 293204.064 & 288.8 & 21.79 & $\dagger$ & & & \\
\hline
\end{tabular}


A\&A 582, L1 (2015)

Table A.2. continued.

\begin{tabular}{|c|c|c|c|c|c|c|c|c|}
\hline Species & $\begin{array}{c}\text { Transition } \\
J_{K_{\mathrm{a}}, K_{\mathrm{c}}}-J_{K_{\mathrm{a}}^{\prime}, K_{\mathrm{c}}^{\prime}}^{\prime}\end{array}$ & $\begin{array}{c}\text { Predicted } \\
\text { frequency }(\mathrm{MHz})\end{array}$ & $\begin{array}{r}E_{\text {upp }} \\
(\mathrm{K})\end{array}$ & $S_{i j}$ & $\begin{array}{c}\text { Observed }^{1} \\
\text { frequency }(\mathrm{MHz})\end{array}$ & $\begin{array}{c}\text { Observed }^{1} \\
T_{\mathrm{MB}}(\mathrm{K})\end{array}$ & $\begin{array}{c}\text { Model }^{2} \\
T_{\mathrm{MB}}(\mathrm{K})\end{array}$ & Blends \\
\hline tEME-EE' & $38_{1,37}-37_{2,36}$ & 293204.064 & 288.8 & 21.79 & $\dot{\dagger}$ & & & \\
\hline tEME-EE' & $35_{2,34}-34_{1,33}$ & 294329.625 & 246.8 & 18.86 & 294330.0 & 0.28 & 0.17 & \\
\hline tEME-EE & $35_{2,34}-34_{1,33}$ & 294329.625 & 246.8 & 18.86 & $\dagger$ & & & \\
\hline tEME-AE & $35_{2,34}-34_{1,33}$ & 294329.673 & 246.8 & 18.86 & $\dagger$ & & & \\
\hline tEME-EA & $35_{2,34}-34_{1,33}$ & 294330.403 & 246.8 & 18.86 & $\dagger$ & & & \\
\hline tEME-AA & $35_{2,34}-34_{1,33}$ & 294330.451 & 246.8 & 18.86 & $\dagger$ & & & \\
\hline tEME-EE & $21_{3,18}-20_{2,19}$ & 294868.623 & 99.8 & 5.34 & $\ldots$ & $\ldots$ & 0.15 & $\mathrm{CH}_{3} \mathrm{OCOH}$ \\
\hline tEME-EE' & $21_{3,18}-20_{2,19}$ & 294868.631 & 99.8 & 5.34 & $\dagger$ & & & \\
\hline tEME-AE & $21_{3,18}-20_{2,19}$ & 294868.926 & 99.8 & 5.34 & $\dagger$ & & & \\
\hline tEME-EE & $37_{2,36}-36_{2,35}$ & 294869.441 & 274.8 & 36.86 & $\grave{\dagger}$ & & & \\
\hline tEME-EE' & $37_{2,36}-36_{2,35}$ & 294869.441 & 274.8 & 36.86 & $\dagger$ & & & \\
\hline tEME-AE & $37_{2,36}-36_{2,35}$ & 294869.456 & 274.8 & 36.86 & $\dagger$ & & & \\
\hline tEME-EA & $37_{2,36}-36_{2,35}$ & 294869.504 & 274.8 & 36.86 & $\dagger$ & & & \\
\hline tEME-AA & $37_{2,36}-36_{2,35}$ & 294869.519 & 274.8 & 36.86 & $\dagger$ & & & \\
\hline tEME-EA & $21_{3,18}-20_{2,19}$ & 294870.971 & 99.8 & 5.34 & $\ldots$ & $\ldots$ & 0.10 & $\mathrm{CH}_{3} \mathrm{OCOH}$ \\
\hline tEME-AA & $21_{3,18}-20_{2,19}$ & 294871.271 & 99.8 & 5.34 & $\dagger$ & & & \\
\hline tEME-AA & $42_{2,40}-41_{3,39}$ & 295213.922 & 357.4 & 14.32 & $\ldots$ & $\ldots$ & 0.03 & $\mathrm{CH}_{3} \mathrm{CN} v_{8}=1$ \\
\hline tEME-EA & $42_{2,40}-41_{3,39}$ & 295213.976 & 357.4 & 14.32 & $\dagger$ & & & \\
\hline tEME-AE & $42_{2,40}-41_{3,39}$ & 295215.469 & 357.4 & 14.32 & $\dagger$ & & & \\
\hline tEME-EE & $42_{2,40}-41_{3,39}$ & 295215.522 & 357.4 & 14.32 & $\dagger$ & & & \\
\hline tEME-EE' & $42_{2,40}-41_{3,39}$ & 295215.522 & 357.4 & 14.32 & $\dagger$ & & & \\
\hline tEME-EE & $10_{5,5}-9_{4,5}$ & 296172.265 & 50.0 & 5.20 & $\ldots$ & $\ldots$ & 0.45 & $\mathrm{SO}_{2}$ \\
\hline tEME-EE' & $10_{5,6}-9_{4,6}$ & 296172.333 & 50.0 & 5.20 & $\dagger$ & & & \\
\hline tEME-EE' & $10_{5,5}-9_{4,5}$ & 296172.462 & 50.0 & 5.20 & $\dagger$ & & & \\
\hline tEME-EE & $10_{5,6}-9_{4,6}$ & 296172.530 & 50.0 & 5.20 & $\dagger$ & & & \\
\hline tEME-AE & $10_{5,5}-9_{4,5}$ & 296172.815 & 50.0 & 5.20 & $\dagger$ & & & \\
\hline tEME-AE & $10_{5,6}-9_{4,6}$ & 296172.884 & 50.0 & 5.20 & $\dagger$ & & & \\
\hline tEME-EA & $10_{5,6}-9_{4,6}$ & 296175.521 & 50.0 & 5.20 & $\ldots$ & $\ldots$ & 0.30 & $\mathrm{SO}_{2}$ \\
\hline tEME-EA & $10_{5,5}-9_{4,5}$ & 296175.717 & 50.0 & 5.20 & $\dagger$ & & & \\
\hline tEME-AA & $10_{5,6}-9_{4,5}$ & 296176.037 & 50.0 & 5.20 & $\dagger$ & & & \\
\hline tEME-AA & $10_{5,5}-9_{4,6}$ & 296176.104 & 50.0 & 5.20 & $\dagger$ & & & \\
\hline tEME-EE' & $16_{4,13}-15_{3,12}$ & 296223.035 & 71.0 & 4.07 & 296224.2 & 0.49 & 0.11 & $\mathrm{CH}_{3} \mathrm{OCOH}$ \\
\hline tEME-AE & $16_{4,13}-15_{3,12}$ & 296224.220 & 71.0 & 4.26 & $\dagger$ & & & \\
\hline tEME-EE & $16_{4,13}-15_{3,12}$ & 296224.598 & 71.0 & 4.49 & $\dagger$ & & & \\
\hline tEME-EA & $16_{4,13}-15_{3,12}$ & 296229.670 & 71.0 & 5.60 & 296230.0 & 0.30 & 0.13 & $\mathrm{CH}_{3} \mathrm{OCOH}$ \\
\hline tEME-AA & $16_{4,13}-15_{3,12}$ & 296230.175 & 71.0 & 5.70 & $\dagger$ & & & \\
\hline tEME-EE & $16_{4,12}-15_{3,12}$ & 296235.284 & 71.0 & 1.21 & $\ldots$ & $\ldots$ & 0.04 & $\mathrm{CH}_{3} \mathrm{CH}_{2} \mathrm{CN} v_{12}=1$ \\
\hline tEME-AE & $16_{4,12}-15_{3,12}$ & 296236.371 & 71.0 & 1.44 & $\dagger$ & & & \\
\hline tEME-EE' & $16_{4,12}-15_{3,12}$ & 296236.725 & 71.0 & 1.63 & $\dagger$ & & & \\
\hline tEME-EE' & $16_{4,13}-15_{3,13}$ & 296440.645 & 71.0 & 1.63 & $\ldots$ & $\ldots$ & 0.04 & $\mathrm{CH}_{3} \mathrm{CH}_{2} \mathrm{CN}$ \\
\hline tEME-AE & $16_{4,13}-15_{3,13}$ & 296441.764 & 71.0 & 1.44 & $\dagger$ & & & \\
\hline tEME-EE & $16_{4,13}-15_{3,13}$ & 296442.086 & 71.0 & 1.21 & $\dagger$ & & & \\
\hline tEME-EE & $16_{4,12}-15_{3,13}$ & 296452.772 & 71.0 & 4.49 & $\ldots$ & $\ldots$ & 0.24 & $\mathrm{CH}_{3} \mathrm{CH}_{2} \mathrm{CN}$ \\
\hline tEME-EA & $16_{4,12}-15_{3,13}$ & 296453.716 & 71.0 & 5.59 & $\dagger$ & & & \\
\hline tEME-AE & $16_{4,12}-15_{3,13}$ & 296453.915 & 71.0 & 4.26 & $\dagger$ & & & \\
\hline tEME-AA & $16_{4,12}-15_{3,13}$ & 296453.976 & 71.0 & 5.70 & $\dagger$ & & & \\
\hline tEME-EE' & $16_{4,12}-15_{3,13}$ & 296454.335 & 71.0 & 4.07 & $\dagger$ & & & \\
\hline tEME-EE' & $24_{3,22}-23_{2,21}$ & 296926.193 & 126.4 & 6.66 & 296926.5 & 0.17 & 0.14 & \\
\hline tEME-EE & $24_{3,22}-23_{2,21}$ & 296926.197 & 126.4 & 6.66 & 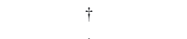 & & & \\
\hline tEME-AE & $24_{3,22}-23_{2,21}$ & 296926.450 & 126.4 & 6.66 & $\dagger$ & & & \\
\hline tEME-EA & $24_{3,22}-23_{2,21}$ & 296928.675 & 126.4 & 6.66 & 296928.7 & 0.42 & 0.09 & $\mathrm{CH}_{3} \mathrm{OCOH} v_{t}=2$ \\
\hline tEME-AA & $24_{3,22}-23_{2,21}$ & 296928.931 & 126.4 & 6.66 & 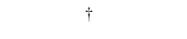 & & & \\
\hline tEME-EA & $38_{0,38}-37_{1,37}$ & 297603.969 & 281.1 & 32.84 & $\ldots$ & $\ldots$ & 0.25 & $\mathrm{CH}_{3} \mathrm{OCOH} v_{t}=2$ \\
\hline tEME-AA & $38_{0,38}-37_{1,37}$ & 297603.974 & 281.1 & 32.84 & $\dagger$ & & & \\
\hline tEME-EE & $38_{0,38}-37_{1,37}$ & 297603.978 & 281.1 & 32.84 & $\dagger$ & & & \\
\hline tEME-EE' & $38_{0,38}-37_{1,37}$ & 297603.978 & 281.1 & 32.84 & $\dagger$ & & & \\
\hline tEME-AE & $38_{0,38}-37_{1,37}$ & 297603.982 & 281.1 & 32.84 & $\dagger$ & & & \\
\hline tEME-EE' & $38_{1,38}-37_{0,37}$ & 298601.597 & 281.1 & 32.85 & $\ldots$ & $\ldots$ & 0.26 & $\mathrm{SO}_{2}$ \\
\hline tEME-EE & $38_{1,38}-37_{0,37}$ & 298601.597 & 281.1 & 32.85 & $\dagger$ & & & \\
\hline tEME-AE & $38_{1,38}-37_{0,37}$ & 298601.604 & 281.1 & 32.85 & $\dagger$ & & & \\
\hline tEME-EA & $38_{1,38}-37_{0,37}$ & 298601.658 & 281.1 & 32.85 & $\dagger$ & & & \\
\hline tEME-AA & $38_{1,38}-37_{0,37}$ & 298601.665 & 281.1 & 32.85 & $\dagger$ & & & \\
\hline tEME-EE & $22_{2,20}-21_{1,21}$ & 300442.750 & 103.1 & 1.73 & $\ldots$ & $\ldots$ & 0.05 & $\mathrm{H}^{13} \mathrm{CCCN}_{v_{7}}=1, \mathrm{CH}_{3} \mathrm{OCOH}$ \\
\hline tEME-EE' & $22_{2,20}-21_{1,21}$ & 300442.750 & 103.1 & 1.73 & $\dagger$ & & & \\
\hline tEME-AE & $22_{2,20}-21_{1,21}$ & 300443.046 & 103.1 & 1.73 & $\dagger$ & & & \\
\hline tEME-EA & $22_{2,20}-21_{1,21}$ & 300444.445 & 103.1 & 1.73 & $\ldots$ & $\ldots$ & 0.03 & $\mathrm{H}^{13} \mathrm{CCCN} v_{7}=1, \mathrm{CH}_{3} \mathrm{OCOH}$ \\
\hline tEME-AA & $22_{2,20}-21_{1,21}$ & 300444.741 & 103.1 & 1.73 & $\dagger$ & & & \\
\hline tEME-EE' & $36_{2,35}-35_{1,34}$ & 300583.963 & 260.6 & 19.88 & 300584.4 & 0.25 & 0.17 & \\
\hline tEME-EE & $36_{2,35}-35_{1,34}$ & 300583.963 & 260.6 & 19.88 & $\dagger$ & & & \\
\hline tEME-AE & $36_{2,35}-35_{1,34}$ & 300584.008 & 260.6 & 19.88 & $\dagger$ & & & \\
\hline tEME-EA & $36_{2,35}-35_{1,34}$ & 300584.687 & 260.6 & 19.88 & $\dagger$ & & & \\
\hline tEME-AA & $36_{2,35}-35_{1,34}$ & 300584.731 & 260.6 & 19.88 & $\dagger$ & & & \\
\hline tEME-AA & $39_{1,38}-38_{2,37}$ & 302148.596 & 303.8 & 22.87 & $\ldots$ & $\ldots$ & 0.14 & $\mathrm{SO}_{2}$ \\
\hline
\end{tabular}


B. Tercero et al.: Trans ethyl methyl ether in space

Table A.2. continued.

\begin{tabular}{|c|c|c|c|c|c|c|c|c|}
\hline Species & $\begin{array}{c}\text { Transition } \\
J_{K_{\mathrm{a}}, K_{\mathrm{c}}}-J_{K_{\mathrm{a}}^{\prime}, K_{\mathrm{c}}^{\prime}}^{\prime}\end{array}$ & $\begin{array}{c}\text { Predicted } \\
\text { frequency }(\mathrm{MHz})\end{array}$ & $\begin{array}{r}E_{\text {upp }} \\
(\mathrm{K})\end{array}$ & $S_{i j}$ & $\begin{array}{c}\text { Observed }^{1} \\
\text { frequency }(\mathrm{MHz})\end{array}$ & $\begin{array}{c}\text { Observed }^{1} \\
T_{\mathrm{MB}}(\mathrm{K})\end{array}$ & $\begin{array}{c}\text { Model }^{2} \\
T_{\mathrm{MB}}(\mathrm{K})\end{array}$ & Blends \\
\hline tEME-EA & $39_{1,38}-38_{2,37}$ & 302148.597 & 303.8 & 22.87 & $\bar{\dagger}$ & & & \\
\hline tEME-AE & $39_{1,38}-38_{2,37}$ & 302148.985 & 303.8 & 22.87 & $\dagger$ & & & \\
\hline tEME-EE & $39_{1,38}-38_{2,37}$ & 302148.986 & 303.8 & 22.87 & $\dagger$ & & & \\
\hline tEME-EE' & $39_{1,38}-38_{2,37}$ & 302148.986 & 303.8 & 22.87 & $\dagger$ & & & \\
\hline tEME-EE' & $25_{3,23}-24_{2,22}$ & 302611.864 & 136.1 & 6.91 & $\ldots$ & $\ldots$ & 0.14 & $\mathrm{CH}_{3} \mathrm{COCH}_{3}$ \\
\hline tEME-EE & $25_{3,23}-24_{2,22}$ & 302611.867 & 136.1 & 6.91 & $\dagger$ & & & \\
\hline tEME-AE & $25_{3,23}-24_{2,22}$ & 302612.114 & 136.1 & 6.91 & $\dagger$ & & & \\
\hline tEME-EA & $25_{3,23}-24_{2,22}$ & 302614.334 & 136.1 & 6.91 & $\ldots$ & $\ldots$ & 0.09 & $\mathrm{CH}_{3} \mathrm{COCH}_{3}$ \\
\hline tEME-AA & $25_{3,23}-24_{2,22}$ & 302614.582 & 136.0 & 6.91 & $\dagger$ & & & \\
\hline tEME-EE' & $17_{4,14}-16_{3,13}$ & 304150.005 & 77.5 & 4.81 & $\ldots$ & $\ldots$ & 0.14 & $\mathrm{CH}_{2} \mathrm{CHCN}$ \\
\hline tEME-AE & $17_{4,14}-16_{3,13}$ & 304151.052 & 77.5 & 4.99 & $\dagger$ & & & \\
\hline tEME-EE & $17_{4,14}-16_{3,13}$ & 304151.281 & 77.5 & 5.19 & $\dagger$ & & & \\
\hline tEME-EA & $17_{4,14}-16_{3,13}$ & 304155.769 & 77.5 & 5.86 & $\ldots$ & $\ldots$ & 0.14 & $\mathrm{CH}_{2} \mathrm{CHCN}$ \\
\hline tEME-AA & $17_{4,14}-16_{3,13}$ & 304156.225 & 77.5 & 5.90 & $\dagger$ & & & \\
\hline tEME-EE & $11_{5,6}-10_{4,6}$ & 304218.289 & 54.3 & 5.39 & $\ldots$ & $\ldots$ & 0.48 & $\mathrm{CH}_{3} \mathrm{OH}$ \\
\hline tEME-EE' & $11_{5,7}-10_{4,7}$ & 304218.360 & 54.3 & 5.39 & $\dagger$ & & & \\
\hline tEME-EE' & $11_{5,6}-10_{4,6}$ & 304218.485 & 54.3 & 5.39 & $\dagger$ & & & \\
\hline tEME-EE & $11_{5,7}-10_{4,7}$ & 304218.556 & 54.3 & 5.39 & $\dagger$ & & & \\
\hline tEME-AE & $11_{5,6}-10_{4,6}$ & 304218.838 & 54.3 & 5.39 & $\dagger$ & & & \\
\hline tEME-AE & $11_{5,7}-10_{4,7}$ & 304218.909 & 54.3 & 5.39 & $\dagger$ & & & \\
\hline tEME-EA & $11_{5,7}-10_{4,7}$ & 304221.544 & 54.3 & 5.38 & $\ldots$ & $\ldots$ & 0.32 & $\mathrm{CH}_{3} \mathrm{OH}$ \\
\hline tEME-EA & $11_{5,6}-10_{4,6}$ & 304221.733 & 54.3 & 5.38 & $\dagger$ & & & \\
\hline tEME-AA & $11_{5,7}-10_{4,6}$ & 304222.011 & 54.3 & 5.39 & $\dagger$ & & & \\
\hline tEME-AA & $11_{5,6}-10_{4,7}$ & 304222.167 & 54.3 & 5.39 & $\dagger$ & & & \\
\hline tEME-EE & $22_{3,19}-21_{2,20}$ & 304228.127 & 108.3 & 5.38 & $\ldots$ & $\ldots$ & 0.14 & $\mathrm{CH}_{3} \mathrm{COCH}_{3}$ \\
\hline tEME-EE' & $22_{3,19}-21_{2,20}$ & 304228.133 & 108.3 & 5.38 & $\dagger$ & & & \\
\hline tEME-AE & $22_{3,19}-21_{2,20}$ & 304228.430 & 108.3 & 5.38 & $\dagger$ & & & \\
\hline tEME-EA & $22_{3,19}-21_{2,20}$ & 304230.454 & 108.3 & 5.38 & $\ldots$ & $\ldots$ & 0.10 & $\mathrm{CH}_{3} \mathrm{COCH}_{3}$ \\
\hline tEME-AA & $22_{3,19}-21_{2,20}$ & 304230.754 & 108.3 & 5.38 & $\dagger$ & & & \\
\hline tEME-EE & $17_{4,13}-16_{3,14}$ & 304481.623 & 77.5 & 5.19 & $\ldots$ & $\ldots$ & 0.25 & $\mathrm{CH}_{3} \mathrm{OCOH} v_{t}=1$ \\
\hline tEME-AE & $17_{4,13}-16_{3,14}$ & 304482.613 & 77.5 & 4.99 & $\dagger$ & & & \\
\hline tEME-EE' & $17_{4,13}-16_{3,14}$ & 304482.900 & 77.5 & 4.81 & $\dagger$ & & & \\
\hline tEME-EA & $17_{4,13}-16_{3,14}$ & 304483.137 & 77.5 & 5.85 & $\dagger$ & & & \\
\hline tEME-AA & $17_{4,13}-16_{3,14}$ & 304483.441 & 77.5 & 5.90 & $\dagger$ & & & \\
\hline tEME-EA & $39_{0,39}-38_{1,38}$ & 305447.899 & 295.7 & 33.86 & 305448.1 & 0.96 & 0.24 & $\mathrm{CH}_{3} \mathrm{CH}_{2} \mathrm{CN} v_{13} / v_{21}$ \\
\hline tEME-EE & $39_{0,39}-38_{1,38}$ & 305447.903 & 295.7 & 33.86 & $\dagger$ & & & \\
\hline tEME-EE' & $39_{0,39}-38_{1,38}$ & 305447.903 & 295.7 & 33.86 & 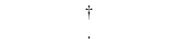 & & & \\
\hline tEME-AA & $39_{0,39}-38_{1,38}$ & 305447.904 & 295.7 & 33.86 & 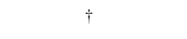 & & & \\
\hline tEME-AE & $39_{0,39}-38_{1,38}$ & 305447.908 & 295.7 & 33.86 & $\dagger$ & & & \\
\hline tEME-EE & $39_{1,39}-38_{0,38}$ & 306290.978 & 295.7 & 33.86 & $\ldots$ & $\ldots$ & 0.24 & $\mathrm{CH}_{3} \mathrm{OH}$ \\
\hline tEME-EE' & $39_{1,39}-38_{0,38}$ & 306290.978 & 295.7 & 33.86 & $\dagger$ & & & \\
\hline tEME-AE & $39_{1,39}-38_{0,38}$ & 306290.985 & 295.7 & 33.86 & $\dagger$ & & & \\
\hline tEME-EA & $39_{1,39}-38_{0,38}$ & 306291.035 & 295.7 & 33.86 & $\dagger$ & & & \\
\hline tEME-AA & $39_{1,39}-38_{0,38}$ & 306291.041 & 295.7 & 33.86 & $\dagger$ & & & \\
\hline tEME-AA & $43_{2,41}-42_{3,40}$ & 306373.059 & 374.1 & 15.22 & $\ldots$ & $\ldots$ & 0.03 & $\mathrm{CH}_{3} \mathrm{OCH}_{3}$ \\
\hline tEME-EA & $43_{2,41}-42_{3,40}$ & 306373.104 & 374.1 & 15.22 & $\dagger$ & & & \\
\hline tEME-AE & $43_{2,41}-42_{3,40}$ & 306374.522 & 374.1 & 15.22 & $\grave{\dagger}$ & & & \\
\hline tEME-EE & $43_{2,41}-42_{3,40}$ & 306374.567 & 374.1 & 15.22 & $\dagger$ & & & \\
\hline tEME-EE' & $43_{2,41}-42_{3,40}$ & 306374.567 & 374.1 & 15.22 & $\dagger$ & & & \\
\hline
\end{tabular}


A\&A 582, L1 (2015)

Table A.3. Detected lines of gauche-trans-n- $\mathrm{CH}_{3} \mathrm{CH}_{2} \mathrm{CH}_{2} \mathrm{OH}$.

\begin{tabular}{|c|c|c|c|c|c|c|c|}
\hline Species & $\begin{array}{c}\text { Transition } \\
J_{K_{\mathrm{a}}, K_{\mathrm{c}}}-J_{K_{\mathrm{a}}^{\prime}, K_{\mathrm{c}}^{\prime}}^{\prime}\end{array}$ & $\begin{array}{c}\text { Predicted } \\
\text { frequency }(\mathrm{MHz})\end{array}$ & $\begin{array}{r}E_{\text {upp }} \\
(\mathrm{K})\end{array}$ & $S_{i j}$ & $\begin{array}{c}\text { Observed } \\
\text { frequency }(\mathrm{MHz})\end{array}$ & $\begin{array}{c}T \\
(\mathrm{~K})\end{array}$ & $\begin{array}{c}\text { Blends/ } \\
\text { Comments }\end{array}$ \\
\hline Gt-n-propanol & $12_{7,6}-12_{6,6}$ & 124417.241 & 58.0 & 4.25 & 124417.1 & 0.04 & $30 \mathrm{~m}$; U-line \\
\hline Gt-n-propanol & $12_{7,5}-12_{6,6}$ & 124417.256 & 58.0 & 4.89 & $\dagger$ & & \\
\hline Gt-n-propanol & $12_{7,6}-12_{6,7}$ & 124418.125 & 58.0 & 4.89 & $124418.2 \dagger$ & 0.06 & $30 \mathrm{~m}$; U-line \\
\hline Gt-n-propanol & $12_{7,5}-12_{6,7}$ & 124418.139 & 58.0 & 4.25 & $\dagger$ & & \\
\hline Gt-n-propanol & $15_{2,14}-14_{1,13}$ & 141219.413 & 55.4 & 9.14 & 141219.8 & 0.04 & $30 \mathrm{~m}$ \\
\hline Gt-n-propanol & $6_{5,2}-5_{4,1}$ & 143143.854 & 21.1 & 4.51 & 143144.6 & 0.10 & $30 \mathrm{~m} ; \mathrm{CH}_{3} \mathrm{CH}_{2} \mathrm{CN} v_{12}=1$ \\
\hline Gt-n-propanol & $6_{5,1}-5_{4,1}$ & 143143.873 & 21.1 & 4.65 & $\dagger$ & & \\
\hline Gt-n-propanol & $6_{5,2}-5_{4,2}$ & 143144.406 & 21.1 & 4.65 & $\dagger$ & & \\
\hline Gt-n-propanol & $6_{5,1}-5_{4,2}$ & 143144.426 & 21.1 & 4.51 & $\dagger$ & & \\
\hline Gt-n-propanol & $8_{7,2}-7_{6,1}$ & 200433.919 & 38.9 & 6.49 & 200434.4 & 0.13 & $30 \mathrm{~m}$ \\
\hline Gt-n-propanol & $8_{7,1}-7_{6,1}$ & 200433.919 & 38.9 & 6.63 & $\dagger$ & & \\
\hline Gt-n-propanol & $8_{7,2}-7_{6,2}$ & 200433.920 & 38.9 & 6.63 & $\dagger$ & & \\
\hline Gt-n-propanol & $8_{7,1}-7_{6,2}$ & 200433.920 & 38.9 & 6.49 & $\dagger$ & & \\
\hline Gt-n-propanol & $23_{11,13}-23_{10,13}$ & 200508.301 & 181.2 & 8.64 & 200508.8 & 0.07 & $30 \mathrm{~m}$ \\
\hline Gt-n-propanol & $23_{11,12}-23_{10,13}$ & 200508.302 & 181.2 & 10.20 & + & & \\
\hline Gt-n-propanol & $23_{11,13}-23_{10,14}$ & 200508.311 & 181.2 & 10.20 & $\dagger$ & & \\
\hline Gt-n-propanol & $23_{11,12}-23_{10,14}$ & 200508.312 & 181.2 & 8.64 & $\dagger$ & & \\
\hline Gt-n-propanol & $9_{7,3}-8_{6,2}$ & 209892.537 & 43.0 & 6.54 & 209892.5 & 0.19 & $30 \mathrm{~m} ; \mathrm{CH}_{2} \mathrm{CHCN} v_{11}=1$ \\
\hline Gt-n-propanol & $9_{7,2}-8_{6,2}$ & 209892.538 & 43.0 & 6.78 & $\dagger$ & & \\
\hline Gt-n-propanol & $9_{7,3}-8_{6,3}$ & 209892.542 & 43.0 & 6.78 & $\dagger$ & & \\
\hline Gt-n-propanol & $9_{7,2}-8_{6,3}$ & 209892.542 & 43.0 & 6.54 & $\dagger$ & & \\
\hline Gt-n-propanol & $24_{0,24}-23_{1,23}$ & 210248.928 & 127.7 & 22.15 & 210249.1 & 0.17 & $30 \mathrm{~m}$ \\
\hline Gt-n-propanol & $24_{1,24}-23_{1,23}$ & 210250.060 & 127.7 & 23.86 & $\dagger$ & & \\
\hline Gt-n-propanol & $24_{0,24}-23_{0,23}$ & 210250.810 & 127.7 & 23.86 & 210252.8 & 0.16 & $30 \mathrm{~m}$ \\
\hline Gt-n-propanol & $24_{1,24}-23_{0,23}$ & 210251.942 & 127.7 & 22.15 & $\dagger$ & & \\
\hline Gt-n-propanol & $44_{12,32}-44_{11,34}$ & 210252.224 & 517.3 & 16.71 & $\dagger$ & & \\
\hline Gt-n-propanol & $37_{12,26}-37_{11,26}$ & 215663.302 & 386.2 & 14.57 & $\ldots$ & $\ldots$ & ALMA; $\mathrm{CH}_{3} \mathrm{CH}_{2} \mathrm{CN} v_{13} / v_{21}$ \\
\hline Gt-n-propanol & $37_{12,25}-37_{11,26}$ & 215663.793 & 386.2 & 19.40 & $\ldots$ & $\ldots$ & " \\
\hline Gt-n-propanol & $37_{12,26}-37_{11,27}$ & 215663.793 & 386.2 & 19.40 & $\ldots$ & $\ldots$ & $"$ \\
\hline Gt-n-propanol & $37_{12,25}-37_{11,27}$ & 215663.793 & 386.2 & 19.40 & $\ldots$ & $\ldots$ & $"$ \\
\hline Gt-n-propanol & $37_{12,26}-37_{11,27}$ & 215672.076 & 386.2 & 19.40 & $\ldots$ & $\ldots$ & $\mathrm{ALMA} ; \mathrm{CH}_{3} \mathrm{COOH} v_{t}=1$ \\
\hline Gt-n-propanol & $37_{12,25}-37_{11,27}$ & 215672.568 & 386.2 & 14.57 & $\ldots$ & $\ldots$ & " \\
\hline Gt-n-propanol & $24_{3,21}-23_{4,20}$ & 216493.373 & 143.6 & 9.36 & $\ldots$ & $\ldots$ & ALMA; $\mathrm{CH}_{3} \mathrm{OCOH}$ \\
\hline Gt-n-propanol & $14_{5,10}-13_{4,9}$ & 217132.672 & 59.3 & 5.22 & $\ldots$ & $\ldots$ & ALMA; $\mathrm{CH}_{3} \mathrm{O}^{13} \mathrm{COH} v_{t}=1, \mathrm{CH}_{3} \mathrm{OD}$ \\
\hline Gt-n-propanol & $34_{12,23}-34_{11,23}$ & 217158.546 & 337.0 & 13.43 & 217159.0 & 0.48 & ALMA; $\mathrm{CH}_{3} \mathrm{COOCH}_{3}$ \\
\hline Gt-n-propanol & $34_{12,22}-34_{11,23}$ & 217158.612 & 337.0 & 17.25 & $\dagger$ & & \\
\hline Gt-n-propanol & $34_{12,23}-34_{11,24}$ & 217159.977 & 337.0 & 17.25 & $\dagger$ & & \\
\hline Gt-n-propanol & $34_{12,22}-34_{11,24}$ & 217160.043 & 337.0 & 13.43 & $\dagger$ & & \\
\hline Gt-n-propanol & $32_{12,21}-32_{11,21}$ & 217935.916 & 306.4 & 12.61 & $\ldots$ & $\ldots$ & ALMA; $\mathrm{CH}_{3} \mathrm{OCH}_{3}, \mathrm{CH}_{3} \mathrm{O}^{13} \mathrm{COH} v_{t}=1$ \\
\hline Gt-n-propanol & $32_{12,20}-32_{11,21}$ & 217935.931 & 306.4 & 15.85 & $\ldots$ & $\ldots$ & " \\
\hline Gt-n-propanol & $32_{12,21}-32_{11,22}$ & 217936.298 & 306.4 & 15.85 & $\ldots$ & $\ldots$ & $"$ \\
\hline Gt-n-propanol & $32_{12,20}-32_{11,22}$ & 217936.314 & 306.4 & 12.61 & $\ldots$ & $\ldots$ & $"$ \\
\hline Gt-n-propanol & $31_{12,20}-31_{11,20}$ & 218268.548 & 291.9 & 12.18 & 218269.0 & 0.84 & ALMA; U-line \\
\hline Gt-n-propanol & $31_{12,19}-31_{11,20}$ & 218268.555 & 291.9 & 12.15 & $\dagger$ & & \\
\hline Gt-n-propanol & $31_{12,20}-31_{11,21}$ & 218268.739 & 291.9 & 12.15 & $\dagger$ & & \\
\hline Gt-n-propanol & $31_{12,19}-31_{11,21}$ & 218268.746 & 291.9 & 12.18 & $\dagger$ & & \\
\hline Gt-n-propanol & $30_{12,19}-30_{11,19}$ & 218567.524 & 277.7 & 11.74 & $\ldots$ & $\ldots$ & ALMA; $\mathrm{CH}_{3} \mathrm{O}^{13} \mathrm{COH} v_{t}=1$ \\
\hline Gt-n-propanol & $30_{12,18}-30_{11,19}$ & 218567.527 & 277.7 & 14.45 & $\ldots$ & $\ldots$ & $"$ \\
\hline Gt-n-propanol & $30_{12,19}-30_{11,20}$ & 218567.616 & 277.7 & 14.45 & $\ldots$ & $\ldots$ & " \\
\hline Gt-n-propanol & $30_{12,18}-30_{11,20}$ & 218567.619 & 277.7 & 11.74 & $\ldots$ & $\ldots$ & " \\
\hline Gt-n-propanol & $29_{12,18}-29_{11,18}$ & 218835.376 & 264.1 & 11.28 & 218835.5 & 1.05 & ALMA; $\mathrm{CH}_{3}^{18} \mathrm{OCOH}$ \\
\hline Gt-n-propanol & $29_{12,17}-29_{11,18}$ & 218835.377 & 264.1 & 13.76 & $\dagger$ & & \\
\hline Gt-n-propanol & $29_{12,18}-29_{11,19}$ & 218835.419 & 264.1 & 13.76 & $\dagger$ & & \\
\hline Gt-n-propanol & $29_{12,17}-29_{11,19}$ & 218835.421 & 264.1 & 11.28 & $\dagger$ & & \\
\hline Gt-n-propanol & $25_{0,25}-24_{1,24}$ & 218880.233 & 138.2 & 23.15 & 218881.2 & 0.80 & ALMA \\
\hline Gt-n-propanol & $25_{1,25}-24_{1,24}$ & 218880.912 & 138.2 & 24.86 & $\dagger$ & & \\
\hline Gt-n-propanol & $25_{0,25}-24_{0,24}$ & 218881.365 & 138.2 & 24.86 & $\dagger$ & & \\
\hline Gt-n-propanol & $25_{1,25}-24_{0,24}$ & 218882.044 & 138.2 & 23.15 & $\dagger$ & & \\
\hline Gt-n-propanol & $12_{6,7}-11_{5,6}$ & 218945.655 & 52.0 & 5.95 & 218946.4 & 0.73 & ALMA \\
\hline Gt-n-propanol & $12_{6,6}-11_{5,6}$ & 218946.539 & 52.0 & 6.77 & $\dagger$ & & \\
\hline Gt-n-propanol & $12_{6,7}-11_{5,7}$ & 218959.585 & 52.0 & 6.77 & 218960.4 & 0.76 & ALMA \\
\hline Gt-n-propanol & $16_{3,14}-15_{2,14}$ & 218960.392 & 65.9 & 5.29 & $\dagger$ & & \\
\hline Gt-n-propanol & $12_{6,6}-11_{5,7}$ & 218960.468 & 52.0 & 5.95 & $\dagger$ & & \\
\hline Gt-n-propanol & $26_{4,22}-25_{5,21}$ & 219065.066 & 170.6 & 6.54 & $\ldots$ & $\ldots$ & $\mathrm{ALMA} ; \mathrm{CH}_{3} \mathrm{OCOH} v_{t}=1$ \\
\hline Gt-n-propanol & $28_{12,17}-28_{11,17}$ & 219074.469 & 250.9 & 10.82 & $\ldots$ & $\ldots$ & ALMA; $\mathrm{CH}_{3} \mathrm{OCOH} v_{t}=1$ \\
\hline Gt-n-propanol & $28_{12,16}-28_{11,17}$ & 219074.470 & 250.9 & 13.07 & $\ldots$ & $\ldots$ & $"$ \\
\hline
\end{tabular}

Notes. Lines of gauche-trans-n- $\mathrm{CH}_{3} \mathrm{CH}_{2} \mathrm{CH}_{2} \mathrm{OH}$ (Gt-n-propanol) ground state present in the spectral scan of Orion KL from the IRAM-30 $\mathrm{m}$ telescope and the ALMA interferometer. Col. 1 indicates the species, Col. 2 gives the transition, Col. 3 the predicted frequency, Col. 4 upper level energy, Col. 5 the line strength, Col. 6 observed frequency at the peak channel of the line (relative to a $v_{\text {LSR }}$ of $+8.0 \mathrm{~km} \mathrm{~s}^{-1}$ ), Col. 7 temperature at the peak channel of the line (main beam temperature for the IRAM data), and Col. 8 shows blends with other molecular species and comments. (†) Blended with previous line. 
Table A.3. continued.

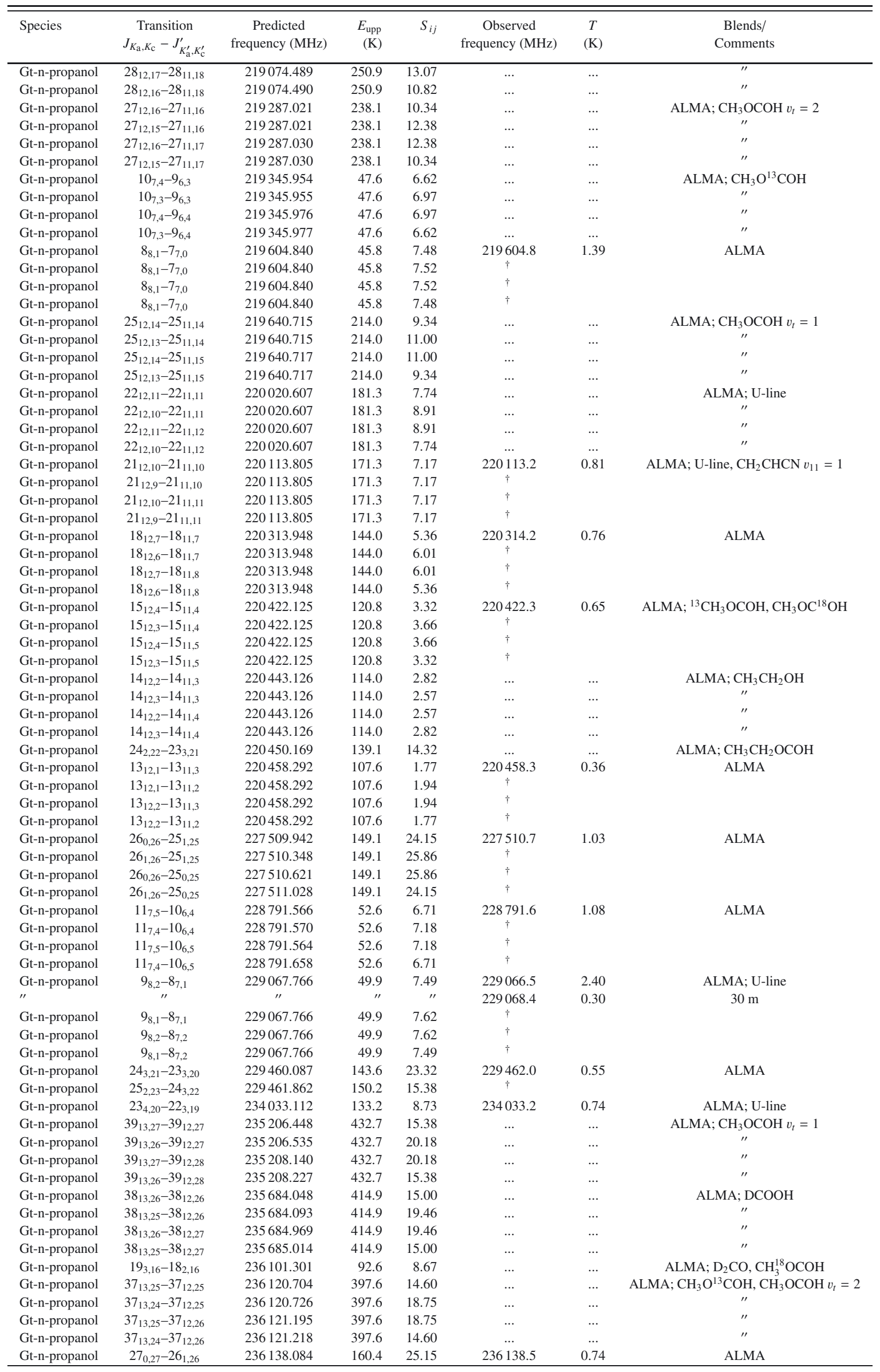


A\&A 582, L1 (2015)

Table A.3. continued.

\begin{tabular}{|c|c|c|c|c|c|c|c|}
\hline Species & $\begin{array}{c}\text { Transition } \\
J_{K_{\mathrm{a}}, K_{\mathrm{c}}}-J_{K_{\mathrm{a}}^{\prime}, K_{\mathrm{c}}^{\prime}}^{\prime}\end{array}$ & $\begin{array}{c}\text { Predicted } \\
\text { frequency }(\mathrm{MHz})\end{array}$ & $\begin{array}{r}E_{\text {upp }} \\
(\mathrm{K})\end{array}$ & $S_{i j}$ & $\begin{array}{c}\text { Observed } \\
\text { frequency }(\mathrm{MHz})\end{array}$ & $\begin{array}{c}T \\
(\mathrm{~K})\end{array}$ & $\begin{array}{c}\text { Blends/ } \\
\text { Comments }\end{array}$ \\
\hline Gt-n-propanol & $27_{1,27}-26_{1,26}$ & 236138.327 & 160.4 & 26.86 & $\dagger^{\dagger}$ & & \\
\hline Gt-n-propanol & $27_{1,27}-26_{0,26}$ & 236138.733 & 160.4 & 25.15 & $\dagger$ & & \\
\hline Gt-n-propanol & $17_{2,16}-16_{1,16}$ & 236879.561 & 69.9 & 2.82 & $\ldots$ & $\ldots$ & ALMA; $\mathrm{CH}_{3} \mathrm{OCOH}$ \\
\hline Gt-n-propanol & $35_{13,23}-35_{12,23}$ & 236882.213 & 364.3 & 13.78 & $\ldots$ & $\ldots$ & $"$ \\
\hline Gt-n-propanol & $35_{13,22}-35_{12,24}$ & 236882.350 & 364.3 & 13.78 & $\ldots$ & $\ldots$ & $"$ \\
\hline Gt-n-propanol & $34_{13,22}-34_{12,22}$ & 237212.075 & 348.4 & 13.36 & $\ldots$ & $\ldots$ & $\mathrm{ALMA} ; \mathrm{CH}_{3} \mathrm{OCOH} v_{t}=2$ \\
\hline Gt-n-propanol & $34_{13,21}-34_{12,22}$ & 237212.077 & 348.4 & 16.65 & $\ldots$ & $\ldots$ & " \\
\hline Gt-n-propanol & $34_{13,22}-34_{12,23}$ & 237212.141 & 348.4 & 16.65 & $\ldots$ & $\ldots$ & " \\
\hline Gt-n-propanol & $34_{13,21}-34_{12,23}$ & 237212.143 & 348.4 & 13.36 & $\ldots$ & $\ldots$ & " \\
\hline Gt-n-propanol & $14_{6,9}-13_{5,9}$ & 237691.949 & 64.3 & 7.37 & 237692.7 & 0.62 & ALMA $;{ }^{13} \mathrm{CH}_{3} \mathrm{OCOH}$ \\
\hline Gt-n-propanol & $32_{13,20}-32_{12,21}$ & 237781.319 & 317.9 & 15.26 & $\ldots$ & $\ldots$ & $"$ \\
\hline Gt-n-propanol & $32_{13,19}-32_{12,21}$ & 237781.319 & 317.9 & 12.47 & $\ldots$ & $\ldots$ & $"$ \\
\hline Gt-n-propanol & $31_{13,19}-31_{12,19}$ & 238024.778 & 303.3 & 12.01 & $\ldots$ & $\ldots$ & $\mathrm{ALMA} ; \mathrm{CH}_{3} \mathrm{OCOH} v_{t}=1$ \\
\hline Gt-n-propanol & $31_{13,18}-31_{12,19}$ & 238024.778 & 303.3 & 14.57 & $\ldots$ & $\ldots$ & " \\
\hline Gt-n-propanol & $31_{13,19}-31_{12,20}$ & 238024.785 & 303.3 & 14.57 & $\ldots$ & $\ldots$ & $"$ \\
\hline Gt-n-propanol & $31_{13,18}-31_{12,20}$ & 238024.785 & 303.3 & 12.01 & $\ldots$ & $\ldots$ & $"$ \\
\hline Gt-n-propanol & $16_{5,11}-15_{4,12}$ & 238051.137 & 73.5 & 5.21 & 238051.4 & 0.64 & ALMA \\
\hline Gt-n-propanol & $37_{6,31}-37_{5,33}$ & 238051.929 & 342.5 & 1.83 & $\dagger$ & $"$ & " \\
\hline Gt-n-propanol & $12_{7,6}-11_{6,5}$ & 238226.322 & 58.0 & 6.81 & $\ldots$ & $\ldots$ & $\mathrm{ALMA} ; \mathrm{CH}_{3} \mathrm{OCOH} v_{t}=1$ \\
\hline " & " & " & " & " & 238226.8 & 0.25 & $30 \mathrm{~m} ; \mathrm{CH}_{3} \mathrm{OCOH} v_{t}=1$ \\
\hline Gt-n-propanol & $12_{7,5}-11_{6,5}$ & 238226.337 & 58.0 & 7.42 & $\dagger$ & & \\
\hline Gt-n-propanol & $29_{13,16}-29_{12,17}$ & 238438.858 & 275.5 & 13.19 & $\ldots$ & $\ldots$ & " \\
\hline Gt-n-propanol & $29_{13,17}-29_{12,18}$ & 238438.859 & 275.5 & 13.19 & $\ldots$ & $\ldots$ & $"$ \\
\hline Gt-n-propanol & $29_{13,16}-29_{12,18}$ & 238438.859 & 275.5 & 11.05 & $\ldots$ & $\ldots$ & " \\
\hline Gt-n-propanol & $10_{8,3}-9_{7,2}$ & 238528.368 & 54.5 & 7.53 & 238528.6 & 1.01 & ALMA \\
\hline " & " & " & " & " & 238528.8 & 0.18 & $30 \mathrm{~m}$ \\
\hline Gt-n-propanol & $10_{8,2}-9_{7,2}$ & 238528.368 & 54.5 & 7.76 & $\dagger$ & & \\
\hline Gt-n-propanol & $10_{8,3}-9_{7,3}$ & 238528.368 & 54.5 & 7.76 & $\dagger$ & & \\
\hline Gt-n-propanol & $10_{8,2}-9_{7,3}$ & 238528.368 & 54.5 & 7.53 & $\dagger$ & & \\
\hline Gt-n-propanol & $23_{13,11}-23_{12,11}$ & 239215.407 & 203.2 & 7.87 & $\ldots$ & $\ldots$ & ALMA; $\mathrm{CH}_{3}{ }^{18} \mathrm{OCOH}$ \\
\hline Gt-n-propanol & $23_{13,10}-23_{12,11}$ & 239215.407 & 203.2 & 9.01 & $\ldots$ & $\ldots$ & $"$ \\
\hline Gt-n-propanol & $23_{13,11}-23_{12,12}$ & 239215.407 & 203.2 & 9.01 & $\ldots$ & $\ldots$ & $"$ \\
\hline Gt-n-propanol & $23_{13,10}-23_{12,12}$ & 239215.407 & 203.2 & 7.87 & $\ldots$ & $\ldots$ & " \\
\hline Gt-n-propanol & $22_{13,10}-22_{12,10}$ & 239291.987 & 192.7 & 7.29 & $\ldots$ & $\ldots$ & $\mathrm{ALMA} ; \mathrm{CH}_{3} \mathrm{OCOH} v_{t}=1$ \\
\hline Gt-n-propanol & $22_{13,9}-22_{12,10}$ & 239291.987 & 192.7 & 8.29 & $\ldots$ & $\ldots$ & " \\
\hline Gt-n-propanol & $22_{13,10}-22_{12,11}$ & 239291.987 & 192.7 & 8.29 & $\ldots$ & $\ldots$ & $"$ \\
\hline Gt-n-propanol & $22_{13,9}-22_{12,11}$ & 239291.987 & 192.7 & 7.29 & $\ldots$ & $\ldots$ & " \\
\hline Gt-n-propanol & $24_{4,21}-23_{3,20}$ & 239313.697 & 144.1 & 9.74 & 239313.9 & 0.68 & $\mathrm{ALMA} ; \mathrm{CH}_{3} \mathrm{OCOH} v_{t}=2$ \\
\hline Gt-n-propanol & $21_{13,9}-21_{12,9}$ & 239356.844 & 182.7 & 6.69 & 239357.4 & 0.26 & ALMA \\
\hline Gt-n-propanol & $21_{13,8}-21_{12,9}$ & 239356.844 & 182.7 & 7.56 & $\dagger$ & & \\
\hline Gt-n-propanol & $21_{13,9}-21_{12,10}$ & 239356.844 & 182.7 & 7.56 & $\dagger$ & & \\
\hline Gt-n-propanol & $21_{13,8}-21_{12,10}$ & 239356.844 & 182.7 & 6.69 & $\dagger$ & & \\
\hline Gt-n-propanol & $20_{13,8}-20_{12,8}$ & 239411.108 & 173.2 & 6.07 & 239411.6 & 0.65 & ALMA; U-line \\
\hline Gt-n-propanol & $20_{13,7}-20_{12,8}$ & 239411.108 & 173.2 & 6.83 & $\dagger$ & & \\
\hline Gt-n-propanol & $20_{13,8}-20_{12,9}$ & 239411.108 & 173.2 & 6.83 & $\dagger$ & & \\
\hline
\end{tabular}


B. Tercero et al.: Trans ethyl methyl ether in space

Table A.3. continued.

\begin{tabular}{|c|c|c|c|c|c|c|c|}
\hline Species & $\begin{array}{c}\text { Transition } \\
J_{K_{\mathrm{a}}, K_{\mathrm{c}}}-J_{K_{\mathrm{a}}^{\prime}, K_{\mathrm{c}}^{\prime}}^{\prime}\end{array}$ & $\begin{array}{c}\text { Predicted } \\
\text { frequency (MHz) }\end{array}$ & $\begin{array}{r}E_{\text {upp }} \\
(\mathrm{K})\end{array}$ & $S_{i j}$ & $\begin{array}{c}\text { Observed } \\
\text { frequency }(\mathrm{MHz})\end{array}$ & $\begin{array}{c}T \\
(\mathrm{~K})\end{array}$ & $\begin{array}{c}\text { Blends/ } \\
\text { Comments }\end{array}$ \\
\hline Gt-n-propanol & $26_{3,24}-25_{2,23}$ & 239523.360 & 161.7 & 16.45 & 239523.8 & 0.44 & ALMA \\
\hline Gt-n-propanol & $16_{13,3}-16_{12,5}$ & 239542.869 & 139.6 & 3.35 & $\ldots$ & $\ldots$ & $"$ \\
\hline Gt-n-propanol & $16_{13,3}-16_{12,4}$ & 239542.869 & 139.6 & 3.69 & $\ldots$ & $\ldots$ & " \\
\hline Gt-n-propanol & $16_{13,4}-16_{12,4}$ & 239542.869 & 139.6 & 3.35 & $\ldots$ & $\ldots$ & " \\
\hline Gt-n-propanol & $28_{1,28}-27_{1,27}$ & 244764.799 & 172.2 & 27.86 & $\dagger$ & & \\
\hline Gt-n-propanol & $28_{0,28}-27_{0,27}$ & 244764.897 & 172.2 & 27.86 & $\dagger$ & & \\
\hline Gt-n-propanol & $28_{1,28}-27_{0,27}$ & 244765.042 & 172.2 & 26.16 & $\dagger$ & & \\
\hline Gt-n-propanol & $25_{4,22}-24_{3,21}$ & 245104.539 & 155.3 & 10.81 & 245104.4 & 0.44 & ALMA \\
\hline Gt-n-propanol & $9_{9,1}-8_{8,0}$ & 248228.722 & 57.7 & 8.48 & 248228.5 & 0.14 & $30 \mathrm{~m}$ \\
\hline Gt-n-propanol & $9_{9,0}-8_{8,0}$ & 248228.722 & 57.7 & 8.52 & $\dagger$ & & \\
\hline Gt-n-propanol & $14_{8,7}-13_{7,7}$ & 276312.417 & 77.2 & 8.60 & $\dagger$ & & \\
\hline Gt-n-propanol & $14_{8,6}-13_{7,7}$ & 276312.419 & 77.2 & 7.88 & $\dagger$ & & \\
\hline Gt-n-propanol & $10_{10,1}-9_{9,0}$ & 276842.794 & 71.0 & 9.48 & 276842.8 & 0.10 & $30 \mathrm{~m}$ \\
\hline Gt-n-propanol & $10_{10,0}-9_{9,0}$ & 276842.794 & 71.0 & 9.52 & $\dagger$ & & \\
\hline Gt-n-propanol & $10_{10,1}-9_{9,1}$ & 276842.794 & 71.0 & 9.52 & $\dagger$ & & \\
\hline Gt-n-propanol & $10_{10,0}-9_{9,1}$ & 276842.794 & 71.0 & 9.48 & $\dagger$ & & \\
\hline Gt-n-propanol & $13_{9,5}-12_{8,4}$ & 286069.306 & 78.6 & 8.66 & 286069.5 & 0.10 & $30 \mathrm{~m}$ \\
\hline Gt-n-propanol & $13_{9,4}-12_{8,4}$ & 286069.306 & 78.6 & 9.10 & $\dagger$ & & \\
\hline Gt-n-propanol & $13_{9,5}-12_{8,5}$ & 286069.306 & 78.6 & 9.10 & $\dagger$ & & \\
\hline Gt-n-propanol & $13_{9,4}-12_{8,5}$ & 286069.306 & 78.6 & 8.66 & $\dagger$ & & \\
\hline
\end{tabular}

